Electron Generation and Transport in Intense Relativistic Laser-Plasma Interactions Relevant to Fast Ignition ICF

T. Ma

April 26, 2010 
This document was prepared as an account of work sponsored by an agency of the United States government. Neither the United States government nor Lawrence Livermore National Security, LLC, nor any of their employees makes any warranty, expressed or implied, or assumes any legal liability or responsibility for the accuracy, completeness, or usefulness of any information, apparatus, product, or process disclosed, or represents that its use would not infringe privately owned rights. Reference herein to any specific commercial product, process, or service by trade name, trademark, manufacturer, or otherwise does not necessarily constitute or imply its endorsement, recommendation, or favoring by the United States government or Lawrence Livermore National Security, LLC. The views and opinions of authors expressed herein do not necessarily state or reflect those of the United States government or Lawrence Livermore National Security, LLC, and shall not be used for advertising or product endorsement purposes.

This work performed under the auspices of the U.S. Department of Energy by Lawrence Livermore National Laboratory under Contract DE-AC52-07NA27344. 


\title{
UNIVERSITY OF CALIFORNIA, SAN DIEGO
}

\section{Electron Generation and Transport in Intense Relativistic Laser-Plasma Interactions Relevant to Fast Ignition ICF}

\author{
A dissertation submitted in partial satisfaction of the \\ requirements for the degree \\ Doctor of Philosophy \\ in \\ Engineering Sciences (Aerospace Engineering) \\ by \\ Tammy Yee Wing Ma
}

Committee in charge:

Professor Farhat Beg, Chair

Professor Asoka Mendis

Professor Cliff M. Surko

Professor George Tynan

Professor David Tytler 
Copyright

Tammy Yee Wing Ma, 2010

All rights reserved. 
The dissertation of Tammy Yee Wing Ma is approved, and it is acceptable in quality and form for publication on microfilm:

Chair

University of California, San Diego

2010 


\section{DEDICATION}

To mom and dad. Thank you for your love and support. And to my brother, Timothy. Because I got my Ph.D. before you. 


\section{EPIGRAPH}

Mallory O'Brian: And we went to the moon. Do we really have to go to Mars?

Sam Seaborn: Yes. Mallory O'Brian: Why?

Sam Seaborn: 'Cause it's next. 'Cause we came out of the cave, and we looked over the hill and we saw fire; and we crossed the ocean and we pioneered the West, and we took to the sky.

The history of man is hung on a timeline of exploration and this is what's next.

- The West Wing

Season 2, Episode 9: Galileo

(29 November 2000) 


\section{TABLE OF CONTENTS}

Signature Page ................. . . iii

Dedication ....................... iv

Epigraph ..................... . $\mathrm{v}$

Table of Contents ................. . . vi

List of Figures . . . . . . . . . . . . . . . ix

Acknowledgements . . . . . . . . . . . . xvii

Vita and Publications.............. . xix

Abstract ...............................

Chapter 1 Introduction . . . . . . . . . . . . . . . . . 1

1.1 Basics of Inertial Confinement Fusion with High Powered

Lasers ................... . . . . 2

1.2 Motivation for Fast Ignition . . . . . . . . . . . . . 8

1.3 Cone-Guided Fast Ignition and its Requirements . . . . . 11

1.4 Outline of the Dissertation . . . . . . . . . . . . . . 16

1.5 Role of the author . . . . . . . . . . . . . 18

Chapter 2 Physics of Intense Laser Plasma Interactions . . . . . . . . . . 20

2.1 Introduction . . . . . . . . . . . . . . 20

2.2 Ponderomotive Force . . . . . . . . . . . . . . . . 22

2.3 Laser Interaction with Underdense Plasma . . . . . . . . 24

2.3.1 Self-Focusing and Filamentation . . . . . . . . . 24

2.3.2 Inverse Bremsstrahlung Absorption . . . . . . . . 26

2.4 Laser Interaction with Overdense Plasma . . . . . . . . . 27

2.4.1 Resonance Absorption . . . . . . . . . . . . . 27

2.4.2 Vacuum Heating . . . . . . . . . . . . . . 29

$2.4 .3 \mathrm{j} \times$ B Heating . . . . . . . . . . . . . . 31

2.5 Generation and Transport of Relativistic Electron Beams in Solid Targets . . . . . . . . . . . . . . . . 33

2.5.1 Hot Electron Conversion Efficiency . . . . . . . 34

2.5.2 Hot Electron Temperature Scaling . . . . . . . . . 36

2.5.3 Collisional versus Collective Effects in Propagation 38

2.6 Conclusions . . . . . . . . . . . . . . . . 40 
Chapter 3 The Titan and Vulcan Laser Facilities and Experimental Configurations . . . . . . . . . . . . . . . . . . . 42

3.1 Introduction . . . . . . . . . . . . . . . . 42

3.2 The Technology behind Ultra-Short Pulse, Ultra-High Intensity Lasers . . . . . . . . . . . . . . . . . . 43

3.3 Formation of Laser Prepulse . . . . . . . . . . . . . 46

3.4 The Titan PW OPCPA Laser . . . . . . . . . . . . . 47

3.5 The Vulcan PW OPCPA Laser . . . . . . . . . . . . . . 49

3.6 Experimental Diagnostics . . . . . . . . . . . . . . 50

3.6.1 Characterizing the Laser . . . . . . . . . . . . 51

3.6.1.1 Laser Energy . . . . . . . . . . . . . . 51

3.6.1.2 Laser Focal Spot . . . . . . . . . . . . 51

3.6.1.3 Laser Pulselength . . . . . . . . . . . . . . 52

3.6.1.4 Laser Prepulse . . . . . . . . . . . . . 53

3.6.2 Target Self-Emission Diagnostics . . . . . . . . . . 54

3.6.2.1 XUV Imager . . . . . . . . . . . . . 54

3.6.2.2 XUV Flat Field Spectrometer . . . . . . 55

3.6.2.3 $\mathrm{Cu} \mathrm{K \alpha}$ Imager . . . . . . . . . . . . . . 56

3.6.2.4 Highly Oriented Pyrolytic Graphite (HOPG) Spectrometer . . . . . . . . 62

3.6.2.5 Single Photon Counting CCD . . . . . . 65

3.6.2.6 Cross-Calibration of the $\mathrm{Cu} \mathrm{K} \alpha$ Diagnostics . . . . . . . . . . 66

Chapter 4 Measurement of Electron-Heated Target Temperatures using Extreme Ultraviolet Imaging and Spectroscopy . . . . . . . . . 68

4.1 Theory of Extreme Ultraviolet Radiation . . . . . . . . . 68

4.2 The XUV Imaging Diagnostic . . . . . . . . . . . . . 71

4.2.1 Diagnostic Geometry . . . . . . . . . . . . . . 71

4.2.2 Calibration of Multilayer Mirrors . . . . . . . . . 74

4.2.3 Damage to Mirrors from Exploding Targets . . . 78

4.3 Experimental Results . . . . . . . . . . . . . . . . . . 79

4.4 LASNEX Calculations . . . . . . . . . . . . . . 82

4.5 Discussion: The Use of the XUV Imagers . . . . . . . . . 87

4.6 Spectroscopy in the XUV . . . . . . . . . . . . . . 88

4.6.1 The XUV Flat-Field Spectrometer . . . . . . . . . 88

$4.6 .2 \quad$ Spectroscopy Results . . . . . . . . . . . . . . . . 89

Chapter 5 Transport of Energy by Ultra-Intense Laser-Generated Electrons in Nail-Wire Targets . . . . . . . . . . . . . . . . . . 94

5.1 Introduction . . . . . . . . . . . . . . . . . . 94

5.2 Experimental Setup . . . . . . . . . . . . . . . . . . 95

5.3 Experimental Results and Analysis . . . . . . . . . 98 
5.3.1 K $\alpha$ Emission and Bulk Heating . . . . . . . . . 98

5.3.2 XUV Emission and Surface Heating . . . . . . . . 102

5.4 e-PLAS Simulations . . . . . . . . . . . . . . . . . 104

5.5 Discussion . . . . . . . . . . . . . . . . 110

Chapter 6 Effects of Preplasma on Hot Electron Coupling and Propaga-

tion in Cone-Attached Wire Targets . . . . . . . . . . . . . . . 113

6.1 Introduction . . . . . . . . . . . . . . . 113

6.2 Experimental Setup . . . . . . . . . . . . . . 121

6.3 Experimental Results . . . . . . . . . . . . . . . 125

6.4 HYDRA Modeling of Preplasma Formation in Cone . . . 129

6.5 Inferring the Generated Hot Electron Distribution and Energy with Zuma . . . . . . . . . . . . . 132

6.6 Discussion . . . . . . . . . . . . . . . . . . 141

Chapter $7 \quad$ Conclusions and Future Work . . . . . . . . . . . . . . . . . . 149

7.1 Summary of the Work . . . . . . . . . . . . . . . 149

7.2 Future Experiments and Modeling . . . . . . . . . . . 152

7.3 Concluding Remarks . . . . . . . . . . . . . . . 153

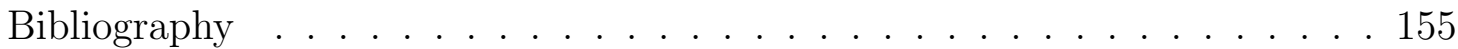




\section{LIST OF FIGURES}

Figure 1.1: The fusion cross sections versus center-of-mass energy for various fusion reactions. The DT reaction has the largest cross section in the entire energy range below $400 \mathrm{keV}$. Figure taken from Atzeni and Meyer-Ter-Vehn, The Physics of Inertial Fusion: Beam Plasma Interaction, Hydrodynamics, Hot Dense Matter (2004)[3] . . . . . . . . . . . . . . . . . . .

Figure 1.2: ICF uses either (a) direct drive or (b) indirect drive to deliver the laser energy to the fuel capsule. . . . . . . . . . . . .

Figure 1.3: In a fusion reaction, power deposited by alpha particles is in competition with radiative losses by bremsstrahlung emission. Here power deposited by alpha particles and power emitted by bremsstrahlung in an equimolar DT plasma are plotted as a function of temperature. For ignition to occur, the DT fusion plasma must therefore exceed a temperature of $4.3 \mathrm{keV}$. Curves taken from expressions in Atzeni, The Physics of Inertial Fusion (2004)[3]. . . . . . . . . . . . . . . . . . . .

Figure 1.4: (a) Energy fraction delivered to the plasma ions for different values of the density. (b) Alpha particle stopping range versus plasma temperature for different values of the density. Figure taken from Fraley, Physics of Fluids (1974)[5] . . . . . . . . 8

Figure 1.5: A schematic of the inertial confinement fast ignition fusion concept. . . . . . . . . . . . . . . .

Figure 1.6: In conventional ICF, the central hot spot ignites a high density cold shell. In fast igntion, the fuel is first isochorically compressed to a moderately high density with no hot spot. Fast electrons generated from a separate high-intensity short pulse laser create a hot spot. . . . . . . . . . . . . . . . . 10

Figure 1.7: Pulse parameters for fast ignition, for particles with a range of $0.6 \mathrm{~g} / \mathrm{cm}^{2}$, as determined from 2-D DUED simulations. (a) Ignition windows in the power-energy plane, and (b) Ignition windows in the intensity-energy plane. Figure taken from Atzeni, Physics of Plasmas (1999)[8]. . . . . . . . . . . . . . . 11

Figure 1.8: Fuel gain as a function of fuel energy. Squares : 2D numerical simulations using DUED, solid line: isochoric model (i.e., fast ignition), broken line: isobaric model (i.e., central hot spot ignition). Figure taken from Atzeni, Physics of Plasmas (1999)[8]. 12

Figure 1.9: Cartoon of the cone-focus geometry for fast ignition. The cone provides an open path for the ignitor beam opposite the implosion. 13 
Figure 1.10: (a) The NIF ignition scale cone-guided FI baseline design. (b) A simulation with a temporal radiation profile as shown (with a $\mathrm{T}_{R, \max }$ of $250 \mathrm{eV}$ ), where the capsule absorbs $\sim 150 \mathrm{~kJ}$ of $\mathrm{x}$-ray energy results in (c) the imploded configuration with a solid-angle-averaged fuel column density $<\rho \mathrm{R}>_{D T}=2.2 \mathrm{~g} / \mathrm{cm}^{2}$. Figure taken from Hatchett, Fusion Science Technology (2006)[12]. 14

Figure 1.11: (a) The reentrant cone target used by Kodama et al. The gold cone was attached to a CD shell of $500 \mu \mathrm{m}$ diameter and $7 \mu \mathrm{m}$ wall thickness. (b) An x-ray image showing the imploded core plasma at the cone tip. Figure taken from Kodama, Nature

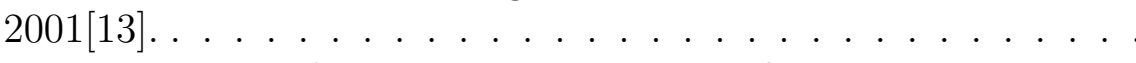

Figure 1.12: The key issues for electron cone-guided fast ignition include tailoring the incident laser profile, understanding the effect of preplasma, optimizing the electron conversion efficiency, characterizing the electron transport, and determining the propagation and stopping of the electrons in the fuel. . . . . . . . . . . 16

Figure 2.1: The mechanism behind self-focusing and filamentation caused by the ponderomotive force. . . . . . . . . . . . . 25

Figure 2.2: A schematic interpretation of resonance absorption. For $p$ polarized light, the electric vector of the light wave lies in the plane of incidence. The electric field can tunnel up the plasma density gradient to launch electron-plasma waves at the critical surface. . . . . . . . . . . . . . . . 28

Figure 2.3: Simplified capacitor model of vacuum heating absorption. . . . 30

Figure 2.4: Conversion efficiency of laser energy into hot electron energy plotted against intensity on target. The 20 and 5 ps points are for laser energies of 200-400 J, and 450 fs data are with 15-20 J energies. Figure taken from Key, Physics of Plasmas 1998[31]. .

Figure 2.5: Predicted electron energy spectrum $T_{\text {hot }}$ based on Ponderomotive (solid, green), Beg (dashed, purple), and Haines (dotted, red) scaling. Experimental evidence seems to show that below laser intensities of $10^{19} \mathrm{~W} / \mathrm{cm}^{2}, T_{\text {hot }}$ scales with Beg or Haines[29, 32], and above that intensity, with Ponderomotive[33]. 37

Figure 3.1: A typical chirped-pulse amplification (CPA) configuration. Image courtesy of LLNL S\&TR of September 1995. . . . . . . . .

Figure 3.2: A typical intensity trace of a full system Titan short pulse laser shot. The ASE pedestal beings approximately 3 ns before the main pulse arrives at $\mathrm{T}=0 \mathrm{~ns}$. The intensity of the main pulse saturates the diode (evidenced by the flat top on the peak), and the gradual drop in peak pulse intensity is due to the finite rise and decay time of the diode. Image courtesy of P. Patel. . . . . 46 
Figure 3.3: A 3-D model of the Titan laser at LLNL. Image courtesy of R. Van Maren. . . . . . . . . . . . . . . . . . . . . 48

Figure 3.4: A 3D model of the Vulcan Laser Facility at the Rutherford Appleton Laboratory. Image courtesy of the Central Laser Facility. 50

Figure 3.5: (a) The Titan OPCPA focal spot at TCC as imaged by the Equivalent Plane Monitor. (b) The integrated power fraction of the focal spot in (a). . . . . . . . . . . . . . . . 51

Figure 3.6: The intensity of the overlap of the split pulse gives the pulse width of the shot. . . . . . . . . . . . . . . 52

Figure 3.7: Oscilloscope trace showing the prepulse profile of a high-power laser pulse. The main pulse is saturated. Figure taken from MacPhee, Review of Scientific Instruments 2008[58]. . . . . . . 53

Figure 3.8: The use of a concave aperiodic grating allows the spectrum to be recorded on a flat plane, rather than on a curved surface matching the Rowland circle when simply using a concave grating. Image courtesy of M. Y. Shen. . . . . . . . . . . . . 55

Figure 3.9: The energy level diagram for copper $(\mathrm{Z}=29)$. K $\alpha$ radiation occurs through a $n=2$ to $n=1$ transition, while $\mathrm{K} \beta$ represents $n=3$ to $n=1) \ldots \ldots \ldots \ldots \ldots \ldots \ldots$

Figure 3.10: The K-shell ionization cross section for copper as a function of overvoltage. The curve is a fit to empirical data as described by Hombourger [64]. . . . . . . . . . . . . . . . 57

Figure 3.11: Schematic of the experimental setup of a $\mathrm{Cu} \mathrm{K} \alpha$ Imager. . . . 58

Figure 3.13: The collection efficiency of the $\mathrm{Cu} \mathrm{K} \alpha$ decreases as a function of temperature due to the shifting and broadening of the $\mathrm{K} \alpha$ line out of the effective bandwidth of the imager diagnostic. Data points are derived by evaluating the ratio of area under the $\mathrm{Cu}$ $\mathrm{K} \alpha$ line emission bounded by the aperture energy bandwidth of the diagnostic. Data courtesy of K. Akli. . . . . . . . . . . . .

Figure 3.12: The shift of the $\mathrm{Cu} \mathrm{K} \alpha$ line as a function of temperature. Spectra as predicted from the 0-D collisional radiative code FLYCHK. 61

Figure 3.14: Schematic of the experimental setup of a HOPG crystal. . . . . 62

Figure 3.15: The intrinsic mosaicity of the HOPG crystal allows for rays emitted by a point source to be focused onto a plane in the image plane if the crystallites are lying on a Rowland circle. This mosaic focusing occurs in the 1:1 magnification geometry, when the focal length $F$ between the source and the crystal, and the crystal and the image plane are equal. When rays are reflected off multiple crystallites at different depths, a focusing error giving the x-ray line width $\Delta s$ will occur. . . . . . . 63

Figure 3.16: Schematic of the experimental setup of a Single Photon Counting $\mathrm{CCD} \ldots \ldots \ldots \ldots \ldots \ldots$ 
Figure 4.1: (a) A $256 \mathrm{eV}$ image of a half sphere with a grid $228 \mu \mathrm{m}$ behind it. (b) A $68 \mathrm{eV}$ image of a half sphere with a grid $804 \mu \mathrm{m}$ behind it. (c) A $68 \mathrm{eV}$ image of a copper cone. (d) A $68 \mathrm{eV}$ image of a $10 \mu m \mathrm{CD} / 5 \mu m \mathrm{Al} / 10 \mu m \mathrm{CD}$ sandwich. . . . . . . . . . 70

Figure 4.2: Schematic of XUV imaging diagnostic setup. . . . . . . . . . . 72

Figure 4.3: Schematic of multilayer reflector of $n$ bilayer pairs. The parameters $\lambda, \theta$, and $d$ are chosen to satisfy the Bragg equation, and the relative thicknesses of the high- and low-Z materials are chosen to optimize reflectivity. . . . . . . . . . . .

Figure 4.4: A schematic of the LBL Center for X-ray Optics Beamline 6.3.2 at the Advanced Light Source which was used for the calibration of the XUV multilayer mirrors. Figure taken from Underwood, Review of Scientific Instruments (1996)[80]. . . . . . . . . . .

Figure 4.5: (a) Reflectivity as a function of energy at various grazing incidence angles for the $256 \mathrm{eV}$ spherical and turning mirrors. Open purple squares: spherical mirror at $83^{\circ}$ incidence angle; open blue triangles: spherical mirror at $85^{\circ}$ incidence angle; solid orange circles: turning mirror at $43^{\circ}$ incidence angle; yellow hatches: turning mirror at $45^{\circ}$ angle. (b) Reflectivity as a function of energy at various grazing incidence angles for the 68 eV spherical and turning mirrors. Open green diamonds: spherical mirror at $75^{\circ}$ incidence angle; open blue triangles: spherical mirror at $85^{\circ}$ incidence angle; solid orange circles: turning mirror at $43^{\circ}$ incidence angle; yellow hatches: turning mirror at $45^{\circ}$ incidence angle. . . . . . . . . . . . . . .

Figure 4.6: Comparison of reflectivity for different orders on the $68 \mathrm{eV}$ spherical mirror. All measurements were taken at $85^{\circ}$ grazing angle incidence. Green crosses: first order; red dotted line: second order; blue solid triangles: fourth order. . . . . . . . . .

Figure 4.7: Effect of accumulation of debris on the mirror surface due to shot exposure, plotted as reflectivity as a function of number of shots. Green dotted line with diamonds is for the $68 \mathrm{eV}$ XUV spherical multilayer mirror and the purple solid line with circles is for the $256 \mathrm{eV}$ XUV spherical multilayer mirror. Error bars for the $256 \mathrm{eV}$ mirror are too small to be seen over the data marker itself. . . . . . . . . . . . . . . . . . . 78

Figure 4.8: A $10 \mu \mathrm{m} \mathrm{Cu}$ target. (a) The raw $256 \mathrm{eV}$ image shown as CCD counts and lineout of the image; (b) the raw $68 \mathrm{eV}$ image shown as CCD counts and lineout of the $68 \mathrm{eV}$ image. The long time scale of bright emission at the $68 \mathrm{eV}$ energy allows the capture of the whole interaction region. . . . . . . . . . . . 
Figure 4.9: LASNEX simulation results: peak target pre-expansion temperature vs absolute brightness for a selection of flat foil targets. Solid pink diamonds: $25 \mu \mathrm{m} \mathrm{Cu}$ at $256 \mathrm{eV}$; solid orange squares: $25 \mu \mathrm{m} \mathrm{Cu}$ at $68 \mathrm{eV}$; open blue circles: $10 \mu \mathrm{m} \mathrm{Cu}$ at $256 \mathrm{eV}$; open green triangles: $10 \mu \mathrm{m} \mathrm{Cu}$ at $68 \mathrm{eV}$; large dashed vertical line: $256 \mathrm{eV}$ detection threshold; small dashed vertical line: 68 eV detection threshold. . . . . . . . . . . . . .

Figure 4.10: (a) Temperature map and lineout for the $10 \mu \mathrm{m} \mathrm{Cu}$ target based on the $256 \mathrm{eV}$ XUV imager. (b) Temperature map and lineout for the $10 \mu \mathrm{m} \mathrm{Cu}$ target based on the $68 \mathrm{eV}$ XUV imager. 84

Figure 4.11: Peak temperatures derived from the two different XUV imagers plotted against each other. Squares: $25 \mu \mathrm{m} \mathrm{Cu}$; circles: $10 \mu \mathrm{m}$ $\mathrm{Cu}$; triangles: $10 \mu \mathrm{m} \mathrm{Al} / 30 \mu \mathrm{m} \mathrm{Cu}$. The diagonal line indicates equivalent temperatures from both imagers. . . . . . . . . .

Figure 4.12: Target backside peak temperatures as a function of target equivalent $\mathrm{Cu}$ density thickness. Triangles: $25 \mu \mathrm{m} \mathrm{Cu}$; circles: $10 \mu \mathrm{m} \mathrm{Cu}$; squares: $10 \mu \mathrm{m} \mathrm{Al} / 30 \mu \mathrm{m} \mathrm{Cu}$. . . . . . . . . . . 86

Figure 4.13: Schematic of flat field spectrometer setup. . . . . . . . . . . . 89

Figure 4.14: (a) The soft x-ray spectra for a CD planar target using a 1200 lines/mm diffraction grating. (b) Schematic illustration of the spatial zone from which plasma emission was recorded. (c) The lineouts for the front and rear surface spectra. . . . . . . . .

Figure 4.15: The comparison of measured experimental line ratios for spatial zone marked in Fig. 4.14 with expected line pair ratios generated using the FLYCHK spectroscopic code. . . . . . . . . . . .

Figure 5.1: Layout of experimental setup showing diagnostic view angles relative to the nail-wire target axis. . . . . . . . . . . .

Figure 5.2: Cross-calibration of Single Photon Counting Camera K $\alpha$ yield vs. HOPG yield to determine absolute number of $\mathrm{Cu} \mathrm{K} \alpha$ values from $\mathrm{Cu} \mathrm{K} \alpha 2-\mathrm{D}$ images. Dotted line is the least squares fit to the open circles (denoting Single Photon Counting Camera yields plotted against HOPG yields). Solid line is least squares fit to the solid circles (denoting improved Single Photon yields plotted against $\mathrm{K} \alpha$ Imager yields. . . . . . . . . . . . . . . . . . 97

Figure 5.3: $\mathrm{Cu} \mathrm{K} \alpha$ emission from the nail-wire target shown with enhanced color scale. . . . . . . . . . . . . . . . .

$\mathrm{K} \alpha / \mathrm{sr} / \mu \mathrm{m}$ for the nail-wire: experimental (connected dotted line), 1D numerical model fit to experimental data (dashed), 1D numerical model inferred total $\mathrm{K} \alpha$ emission profile (before any temperature reduction) (solid). The wire to nail head junction is at $0 \mu \mathrm{m}$ on the abcissa scale. . . . . . . . . . . . . 100 
Figure 5.5: (a) Extreme ultraviolet image (at $68 \mathrm{eV}$ ) of the nail-wire. Emission can be seen all along the nail-wire, and even around the bend. Inset: Schematic showing illumination geometry. (b) Extreme ultraviolet image (at $256 \mathrm{eV}$ emission) of the nail-wire. Dotted line denotes position of lineout in (c) Transverse lineout of wire in $256 \mathrm{eV}$ XUV image which displays prominent limb brightening . . . . . . . . . . . . . . . 103

Figure 5.6: (a) e-PLAS hot electron density plot at 2 ps. (b) Lineouts of the transverse averages for $\mathrm{n}_{h}$ (red), $\mathrm{n}_{c}$ (blue), and $\mathrm{Zn}_{i}$ (brown). The $\mathrm{n}_{h}$ profile shows a near-exponential falloff along the full length of the wire. The $1 / e$ length from e-PLAS is $\sim 76 \mu \mathrm{m} . \quad$. . 106

Figure 5.7: (a) e-PLAS thermal (cold) electron contours at 2 ps. Plot displays enhanced surface heating along the edges of the target. (b) Lineouts of the temperature profiles at 2 ps for hot electrons (red), cold electrons (blue), and ions (brown). Beyond $\mathrm{z}=225$ $\mu \mathrm{m}$ (125 $\mu \mathrm{m}$ along the wire), both the cold electrons and the ions are at a similar temperature of $200 \mathrm{eV}$. (The target starts out with an initial temperature of $100 \mathrm{eV}$.) (c) Transverse average density profiles across the wire at $\mathrm{z}=120 \mu \mathrm{m}$. The hot electron density is seen to extend to a larger radius than the cold electron or ion densities. This correlates to the large flux of hot electrons surfing along the outside edge of the wire. (d) Transverse temperature profiles for the wire at $\mathrm{z}=120 \mu \mathrm{m} . \mathrm{T}_{c}$ is seen to peak just at the edge of the wire. . . . . . . . . . . 108

Figure 5.8: (a) e-PLAS magnetic field contours at 2 ps. A strong $\vec{B}$ field is seen all around the nail head, confining electrons. (b) Axial profile of the magnetic field taken at $\mathrm{r}=13 \mu \mathrm{m}$. Fields are largest ( $\sim 50 \mathrm{MG})$ near the laser spot and along the wire surface near the head-wire interface. $\vec{B}$ field sign is generally positive, indicating that the dominant hot electron flow is away from the head, while return current lies just inside the wire surface. . . . 109

Figure 6.1: Even under varied laser focusing conditions (ray tracing shown here for the case of tight focus at the cone tip, and focusing to a point $400 \mu \mathrm{m}$ before the cone tip), the patterns of $\mathrm{Cu} \mathrm{K} \alpha$ fluorescence from the cone target are very similar. . . . . . . . . 114

Figure 6.2: Typical cone-attached target used in the experiment by Baton et al. The Au cone has a $20 \mu \mathrm{m}$ aperture and $30^{\circ}$ total angle. The cone tip is fixed to a square $300 \times 300 \mu \mathrm{m}^{2}$ planar target. Figure taken from Baton et al., Physics of Plasmas (2008)[96]. . 116 
Figure 6.3: $\mathrm{K} \alpha$ images of $\mathrm{Al}(20 \mu \mathrm{m}) / \mathrm{Cu}(20 \mu \mathrm{m}) / \mathrm{Al}(20 \mu \mathrm{m})$ targets irradiated with a $1 \omega_{0}, 0.4 \mathrm{ps}, 300 \mu \mathrm{m}$ defocused, $5 \times 10^{17} \mathrm{~W} / \mathrm{cm}^{2}$ laser with a 2 ns ASE prepulse pedestal. In (a), the foil was irradiated directly, while in (b), a Au cone of $30^{\circ}$ opening angle, $40 \mu \mathrm{m}$ wide tip was attached to the foil. Both images use the same color scale. Figure taken from Baton et al., Physics of Plasmas (2008)[96]. . . . . . . . . . . . . . . . . . . . 117

Figure 6.4: $\mathrm{K} \alpha$ images of targets irradiated by an essentially prepulse-free $2 \omega_{0}, 0.4$ ps laser pulse at best focus, $1 \times 10^{17} \mathrm{~W} / \mathrm{cm}^{2}$ intensity. In (a) a $\mathrm{Cu}(20 \mu \mathrm{m}) / \mathrm{Al}(20 \mu \mathrm{m})$ target is irradiated directly, while in (b), an $\mathrm{Al}(10 \mu \mathrm{m}) / \mathrm{Cu}(20 \mu \mathrm{m}) / \mathrm{Al}(20 \mu \mathrm{m})$ planar target is attached to a $30^{\circ}, 20 \mu \mathrm{m}$ wide tip cone. The laser was focused directly on the target plane. Both images use the same color scale. Note that the appearance of a slightly lower $\mathrm{K} \alpha$ spot signal in part (b) is due to the presence of the additional $10 \mu \mathrm{m}$ thick Al layer. Figure taken from Baton et al., Physics of Plasmas (2008)[96]. . . . . . . . . . . . . . . . . . . . . . . 118

Figure 6.5: $\mathrm{Cu} \mathrm{K} \alpha$ emission from $\mathrm{Cu}$ cone targets irradiated with the Titan $150 \mathrm{~J}, 0.7 \mathrm{ps}$ laser pulse that is preceded by (a) $7.5 \mathrm{~mJ}$ and (b) 100 mJ energy, nanosecond duration prepulse. . . . . . . . . . 119

Figure 6.6: PIC simulation results for cone targets irradiated with two different levels of prepulse: initial electron density [blue-green], laser Poynting flux at time of peak fluence [red], and energy density of above $1 \mathrm{MeV}$ electrons at end of 3 ps simulation run [green-white]. Dashed lines show the geometric focus of the $\mathrm{f} / 3$ laser beam. Figure taken from MacPhee et al., Physical Review

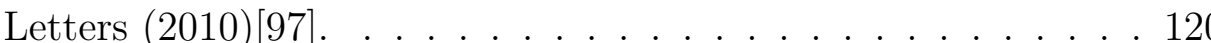

Figure 6.7: The cone-wire target. The $30^{\circ}$ full opening angle $\mathrm{Au}$ cone has $20 \mu \mathrm{m}$ thick walls, and an inner tip diameter of $30 \mu \mathrm{m}$, and thickness of $10 \mu \mathrm{m}$. A Cu wire of $40 \mu \mathrm{m}$ diameter, $1.5 \mathrm{~mm}$ length is glued to the end of the cone. . . . . . . . . . . . . 122

Figure 6.8: Images of the assembled cone-wire target, taken by General Atomics. Each target was individually metrologized to ensure dimensions were within specifications and the gluing of the wire to the cone tip was done well. . . . . . . . . . . . . . . 123

Figure 6.9: Experimental setup showing diagnostic view angles relative to the cone-wire target axis, and representative pieces of data from their respective diagnostic. . . . . . . . . . . . . . . . . . . . . . 124

Figure 6.10: Integrated $\mathrm{K} \alpha$ signal normalized to incident laser energy from cone-wire targets as a function of prepulse energy. . . . . . . . . 126

Figure 6.11: K $\alpha$ profiles taken along the wire (transversely integrated, corrected for view angle and opacity) for varying levels of prepulse. 127 
Figure 6.12: A plot of the integrated $\mathrm{K} \alpha$ yields as derived from the HOPG spectrometer versus the integrated $\mathrm{K} \alpha$ yields from the 2-D spatially resolved $\mathrm{Cu} \mathrm{K} \alpha$ Imager shows that the yields follow a fairly linear trend, indicating that the loss in signal on the imager due to shifting of the $\mathrm{K} \alpha$ line is minimal. . . . . . . . . . . 128

Figure 6.13: HYDRA simulation results of electron density at $3 \mathrm{~ns}$ in a Au cone with (a) $8 \mathrm{~mJ}$ of prepulse and (b) $1 \mathrm{~J}$ of prepulse in the nominal Titan ASE spot and pulselength. Electron density contours of $1 \times 10^{19}, 5 \times 10^{21}$, and $1 \times 10^{22}$ are marked. . . . . . . 130

Figure 6.14: Electron density profiles along the axis of the cone. The relativistic and nonrelativistic critical density locations are marked. 131

Figure 6.15: Resistivity of copper as a function of temperature, as calculated from the Lee-More-Desjarles (LMD) algorithm which is used in the Zuma code. . . . . . . . . . . . . . . . . . . . 133

Figure 6.16: The distribution of electron energy for a $1 \mathrm{MeV}$, 3-D juttnertype relativistic Maxwellian distribution. . . . . . . . . . . . . 134

Figure 6.17: The K $\alpha$ generated along the wire as predicted by Zuma can be transversely integrated over time to give $\mathrm{K} \alpha$ profiles that can be directly compared against the experimental data. The color bar shows the integrated $\mathrm{K} \alpha$ up to 8 ps for a $1.5 \mathrm{~J}, 500 \mathrm{keV}$ $\mathrm{T}_{\text {hot }}$ electron distribution injected into the wire. . . . . . . . . 135

Figure 6.18: Zuma runs of $500 \mathrm{keV}$ and injected electron energies of 1.5, 7.5 , and $15 \mathrm{~J}$ (corresponding to 1,5 , and $10 \%$ conversion of the Titan laser energy). . . . . . . . . . . . . . . 136

Figure 6.19: The K $\alpha$ profiles of Fig.6.18 normalized to a uniform point to display the difference in initial drop off, and the point at which we approach the purely collisional case. . . . . . . . . . . 138

Figure 6.20: Experimental data for a shot with $17 \mathrm{~mJ}$ of prepulse (dotted line) fit with several Zuma simulations (solid lines). . . . . . . . 139

Figure 6.21: Estimated conversion efficiencies from laser energy to hot electron energy as inferred from the best fit Zuma calculation to the experimental data. . . . . . . . . . . . . . . . . . . . . 140

Figure 6.22: Estimated $\mathrm{T}_{\text {hot }}$ for each prepulse shot as inferred from the best fit Zuma calculation to the experimental data. . . . . . . . . . . 141

Figure 6.23: Predicted K $\alpha$ profiles when divergence angle, reflecting boundaries are changed, etc. . . . . . . . . . . . . . . . . . . 144

Figure 6.24: Identical cone-wire targets shot using the $2 \omega$ laser at the Laboratoire pour l'Utilisation des Lasers Intenses (LULI) showed coupling into the wire of approximately a factor of two higher than seen on Titan. This is likely due to the nearly zero prepulse at LULI. . . . . . . . . . . . . . . . . . . . . . . 147 


\section{ACKNOWLEDGEMENTS}

First and foremost, I want to express my deepest gratitude to my academic advisor, Professor Farhat Beg. Not only did he give me the opportunity to do a Ph.D. with him, he has provided me with unending encouragement, support, and guidance the past several years. He has generously given me opportunities to grow as an experimentalist, and to publish papers and attend conferences to learn to communicate the science.

Thank you to my technical and "moral" advisor at Livermore, Dr. Andrew MacPhee, who has always made my grad student years fun, taught me to think on my feet, and trusted me to work independently.

I am hugely indebted to Dr. Mike Key, whose invaluable counsel has guided me through numerous analyses, papers, and presentations.

I am especially grateful to Dr. Prav Patel, who not only fed my coffee addiction, but has provided so much patience and assistance and advice and taught me what it meant to do a good job.

Huge thanks are due to Scott Wilks and David Larson, who taught me / allowed me to work with the HYDRA and Zuma codes, respectively. They were always helpful and patient with me, and allowed me to ask hundreds of dumb questions.

I am appreciative of Andy Mackinnon, Richard Stephens, Harry McLean, and Linn Van Woerkom for their guidance and support as PI's and bosses.

Special thanks to Kramer Akli, Rod Mason, and Steve Hatchett for allowing me to heckle them for simulations (and providing them to me)!

And thank yous all around to a multitude of people I had the privilege of doing experiments with and learning from: Cliff Chen, Sebastien Le Pape, Daniel Hey, Teresa Bartal, Sophie Baton, Sugreev Chawla, Hui Chen, Sophia Chen, Rob Clarke, Becky Daskalova, Laurent Divol, Robert Fedosejevs, Richard Freeman, Hal Friesen, James Green, Drew Higginson, Kerry Highbarger, Charlie Jarrott, Andreas Kemp, Elijah Kemp, James King, Michel Koenig, Andy Krygier, Kate Lancaster, Anthony Link, Derek Mariscal, Chris Murphy, Peter Norreys, Dustin Offermann, Vladimir Ovchinnikov, John Pasley, Frederic Perez, Yuan Ping, Hiroshi Sawada, Riccardo Tommasini, David Turnbull, Ying Tsui, Brad Westover, 
Mingsheng Wei, Linn Van Woerkom, Toshinori Yabuuchi, and Bingbing Zhang.

I am grateful for the generous financial support of the Lawrence Scholar Program.

And finally, to all the professors, colleagues, and friends unmentioned. I thank you for your love and encouragement. Thank you for teaching me and allowing me to learn from you and work with you. Your role in allowing me to be granted a Ph.D. is not overlooked.

This work was performed under the auspices of the U.S. Department of Energy by Lawrence Livermore National Laboratory under contract DE-AC52-07NA27344.

Chapter 4 is a reprint of the material as it appears in T. Ma, Review of Scientific Instruments 79, 093507 (2008); T. Ma, Review of Scientific Instruments 79, 10E312 (2008); and T. Ma, IEEE Transactions on Plasma Science, Vol. 36, No. 4 (2008). The dissertation author was the primary investigator and author of these papers.

Chapter 5, in full, is a reprint of the material as it appears in T. Ma, Physics of Plasmas 16, 112702 (2009). The dissertation author was the primary investigator and author of this paper.

Chapter 6 is material currently being prepared for publication. 


\section{VITA}

2005

2008

2010
B.S. in Engineering and Applied Science (with a concentration in Aeronautics), California Institute of Technology

M.S. in Engineering Sciences (Aerospace Engineering), University of California, San Diego

Ph.D. in Aerospace Engineering, University of California, San Diego

\section{PUBLICATIONS}

T. Ma, et al., "Transport of Energy by Ultra-Intense Laser-Generated Electrons in Nail-Wire Targets," Physics of Plasmas, 16, 112702 (2009).

T. Ma, et al., "Determination of Electron-Heated Temperatures of Petawatt LaserIrradiated Foil Targets with $256 \mathrm{eV}$ and $68 \mathrm{eV}$ Extreme Ultraviolet Imaging," Review of Scientific Instruments, 79, 093507 (2008).

T. Ma, et al., "Electron Heated Target Temperature Measurements in Petawatt Laser Experiments based on Extreme Ultraviolet Imaging and Spectroscopy," Review of Scientific Instruments, 79, 10E312 (2008).

Tammy Ma, et al., "Extreme Ultraviolet Imaging of Electron-Heated Targets in Petawatt Laser Experiments," IEEE Transactions on Plasma Science, Vol. 36, No. 4 (2008).

A. G. MacPhee, et al., "Limitation on Pre-pulse Level for Cone-Guided FastIgnition ICF," Physical Review Letters, 104, 0550002 (2010).

Sebastien Le Pape, et al., "Characterization of the Preformed Plasma for HighIntensity Laser-Plasma Interaction," Optics Letters, Vol 34, No. 19, 2997 (2009).

P.A. Norreys, et al., "Recent Fast Electron Energy Transport Experiments Relevant to Fast Ignition Inertial Fusion," Nuclear Fusion, 49, 104023 (2009).

C. D. Chen, et al., "Bremsstrahlung and Ka Fluorescence Measurements for Inferring Conversion Efficiencies into Fast Ignition Relevant Hot Electrons," Physics of Plasmas, 16, 082705 (2009).

Tsuyoshi Tanimoto, et al., "Measurements of Fast Electron Scaling Generated by Petawatt Laser Systems," Physics of Plasmas, 16, 062703 (2009). 
J. A. King, et al., "Studies on the Transport of High Intensity Laser-Generated Hot Electrons in Cone Coupled Wire Targets", Physics of Plasmas, 16, 020701 (2009).

Sebastien Le Pape, et al., "Density Measurement of Shock Compressed Foam Using 2D X-ray Radiography", Review of Scientific Instruments, 79, 106104 (2008).

A. G. MacPhee, et al., "Diagnostics for Fast Ignition Science", Review of Scientific Instruments, 79, 10F302 (2008).

R. Tommasini, et al., "Development of Backlighting Sources for a Compton Radiography Diagnostic of Inertial Confinement Fusion Targets", Review of Scientific Instruments, 79, 10E901 (2008).

J. Pasley, et al., "Nail-Like Targets for Laser-Plasma Interaction Experiments", IEEE Transactions on Plasma Science, Vol. 36, No. 4 (2008).

K. U. Akli, et al., "Laser Heating of Solid Matter by Light-Pressure-Driven Shocks at Ultrarelativistic Intensities", Physical Review Letters, 100, 165002 (2008).

L. Van Woerkom, et al., "Fast Electron Generation in Cones with Ultraintense Laser Pulses", Physics of Plasmas, 15, 056304 (2008).

J. S. Green, et al., "Effect of Laser Intensity on Fast-Electron-Beam Divergence in Solid-Density Plasmas", Physical Review Letters, 100, 015003 (2008).

J. Pasley, et al., "Experimental Observations of Transport of Picosecond Laser Generated Electrons in a Nail-Like Target", Physics of Plasmas, 14, 120701 (2007). 
ABSTRACT OF THE DISSERTATION

\title{
Electron Generation and Transport in Intense Relativistic Laser-Plasma Interactions Relevant to Fast Ignition ICF
}

\author{
by \\ Tammy Yee Wing Ma \\ Doctor of Philosophy in Engineering Sciences (Aerospace Engineering) \\ University of California San Diego, 2010 \\ Professor Farhat Beg, Chair
}

The reentrant cone approach to Fast Ignition, an advanced Inertial Confinement Fusion scheme, remains one of the most attractive because of the potential to efficiently collect and guide the laser light into the cone tip and direct energetic electrons into the high density core of the fuel. However, in the presence of a preformed plasma, the laser energy is largely absorbed before it can reach the cone tip. Full scale fast ignition laser systems are envisioned to have prepulses ranging between $100 \mathrm{~mJ}$ to $1 \mathrm{~J}$. A few of the imperative issues facing fast ignition, then, are the conversion efficiency with which the laser light is converted to hot electrons, the subsequent transport characteristics of those electrons, and requirements for maximum allowable prepulse this may put on the laser system.

This dissertation examines the laser-to-fast electron conversion efficiency scaling with prepulse for cone-guided fast ignition. Work in developing an extreme ultraviolet imager diagnostic for the temperature measurements of electron-heated targets, as well as the validation of the use of a thin wire for simultaneous determination of electron number density and electron temperature will be discussed. 


\section{Chapter 1}

\section{Introduction}

Achieving ignition and propagating burn in the laboratory through Inertial Confinement Fusion (ICF) would be a tremendous accomplishment that would demonstrate the viability of inertial fusion as an energy option for the world. Towards this goal, the initial National Ignition Facility (NIF) experiments will focus on central hot spot (CHS) ignition, in which a spherical fuel capsule is simultaneously compressed and ignited. This conventional approach, whether with direct or indirect drive, places strict symmetry requirements on the fuel compression and on the uniformity of the driver beams.

Fast Ignition (FI) relaxes these constraints by separating the fuel assembly and heating phases of ICF. Following the compression of the deuterium-tritium fuel by the traditional ICF drivers (with much lower energy than required for CHS ignition), an ultra-intense short pulse laser is brought in to generate a beam of relativistic electrons. These fast electrons propagate through the dense coronal plasma to deposit their energy in the core of the fusion pellet, which creates the hot spot and ignites the fuel. Because this scheme can significantly reduce the energy requirements for compression, and allow for increased flexibility in target smoothness and beam quality and symmetry, FI promises lower ignition thresholds, and therefore higher gains.

The re-entrant cone geometry for fast ignition is a further evolution of the basic FI scheme, in which a hollow cone is embedded in the fuel capsule. The cone would provide an open evacuated path free of coronal plasma for the ignitor laser and 
guide the laser beam closer to the spot to be ignited. The cone may also help concentrate the flow of energetic electrons.

This thesis concentrates on unraveling some of the complex physics involved in the intense laser-plasma interactions relevant to electron cone-guided fast ignition. Understanding and optimizing electron generation and transport are but a few of the many challenges that must be confronted. But these are challenges that the national and international community are more than willing to take on-because while we work towards achieving controlled thermonuclear fusion, we're also exploring new regimes of high energy and density science and pushing the envelope of engineering and technology. Energy from fusion reactions is the most fundamental source of energy in the universe, and if we can successfully harness it here on earth, we will have a nearly limitless source of power.

\subsection{Basics of Inertial Confinement Fusion with High Powered Lasers}

Inertial Confinement Fusion (ICF)[1] is an approach to fusion which relies on the inertia of the target itself to provide confinement. The inertia must hold the mass together for a period of time corresponding to the speed of a sound wave traveling from the surface to the center of the ignited fuel capsule before hydrodynamic expansion quenches the burn. This "confinement time," $\tau$, is generally on the order of a tenth of a nanosecond. This means that incredibly high plasma densities must be achieved in order for a large amount of the fuel to be burned in the short confinement time.

The DT reaction,

$$
\mathrm{D}+\mathrm{T} \rightarrow \alpha(3.52 \mathrm{MeV})+\mathrm{n}(14.06 \mathrm{MeV})
$$

utilizing deuterium and tritium (isotopes of hydrogen) in an equimolar mixture, has the largest fusion cross-section $\left(\sigma_{\max }=5\right.$ barns at $\left.\epsilon \sim 64 \mathrm{keV}\right)$ and reactivity (number of reactions per unit time per unit density) over other candidate reactions up to a temperature of $400 \mathrm{keV}[2]$, so is the primary fuel of interest for fusion energy 
purposes.

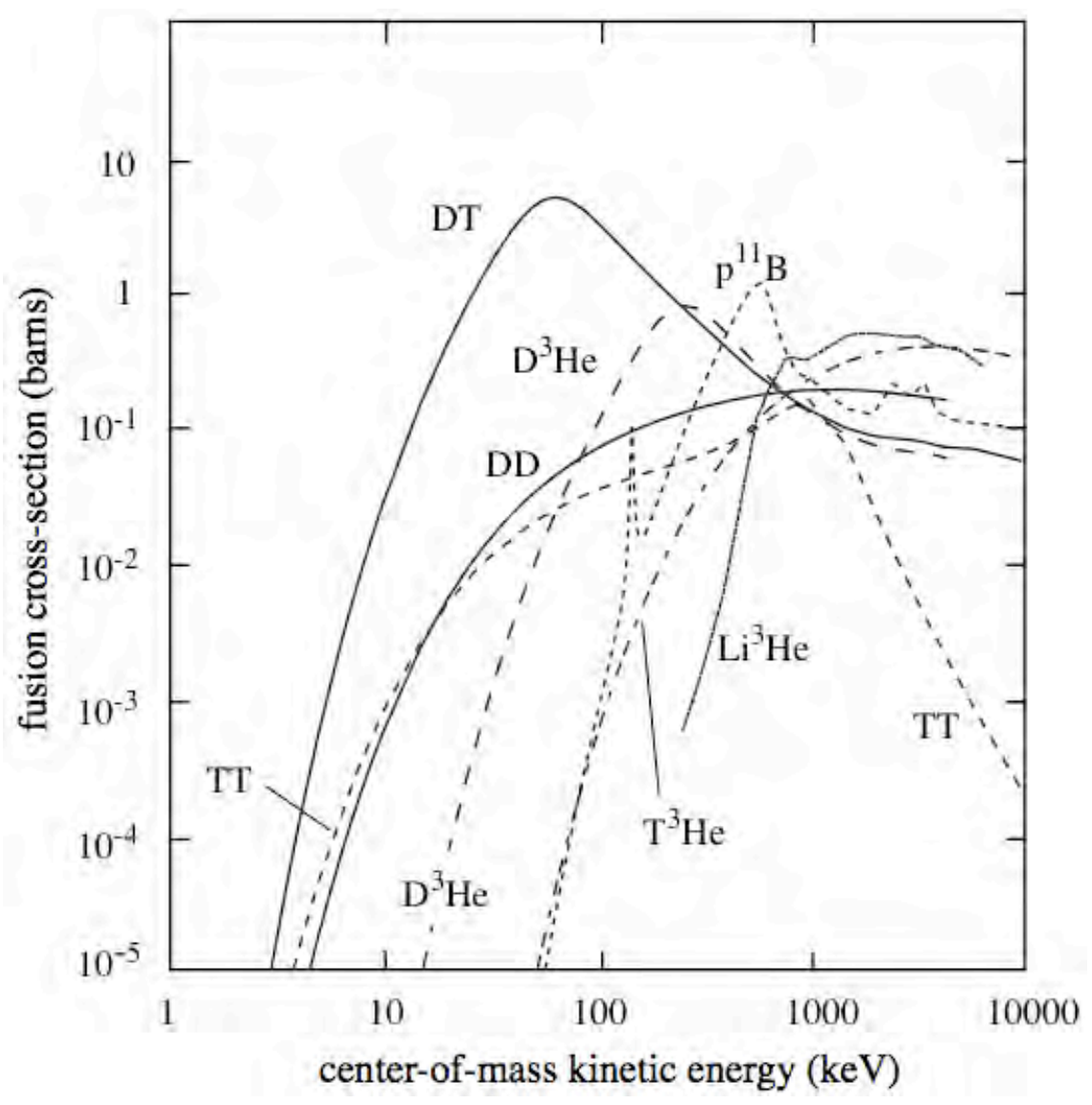

Figure 1.1: The fusion cross sections versus center-of-mass energy for various fusion reactions. The DT reaction has the largest cross section in the entire energy range below $400 \mathrm{keV}$. Figure taken from Atzeni and Meyer-Ter-Vehn, The Physics of Inertial Fusion: Beam Plasma Interaction, Hydrodynamics, Hot Dense Matter (2004)[3].

In ICF, a suitable driver must deliver energy to the DT fuel capsule in a short powerful pulse so that the fuel is compressed to such high temperatures and densities that thermonuclear fusion between the $\mathrm{D}$ and $\mathrm{T}$ atoms become possible. The high-gain DT fuel capsule will generally consist of a spherical shell filled with low density gas $\left(\leq 1.0 \mathrm{mg} / \mathrm{cm}^{3}\right)$. The shell itself will have an outer region that forms the ablator, and an inner region of frozen or liquid DT. The driver may potentially be a high-powered laser or ion beam or Z-pinch. Currently, the most developed and highly pursued method to achieve these conditions is to use an array of energetic 
laser beams, delivering enough energy to drive the compression and implosion.

There are two primary schemes through which the lasers can provide the necessary energy: (1) direct drive, in which lasers are directly incident on the fuel capsule, driving the implosion with their radiation pressure, and (2) indirect drive, where the lasers are first incident on a hohlraum (a high-Z enclosure) which produces x-rays that irradiate the fuel capsule (see Fig. 1.2).
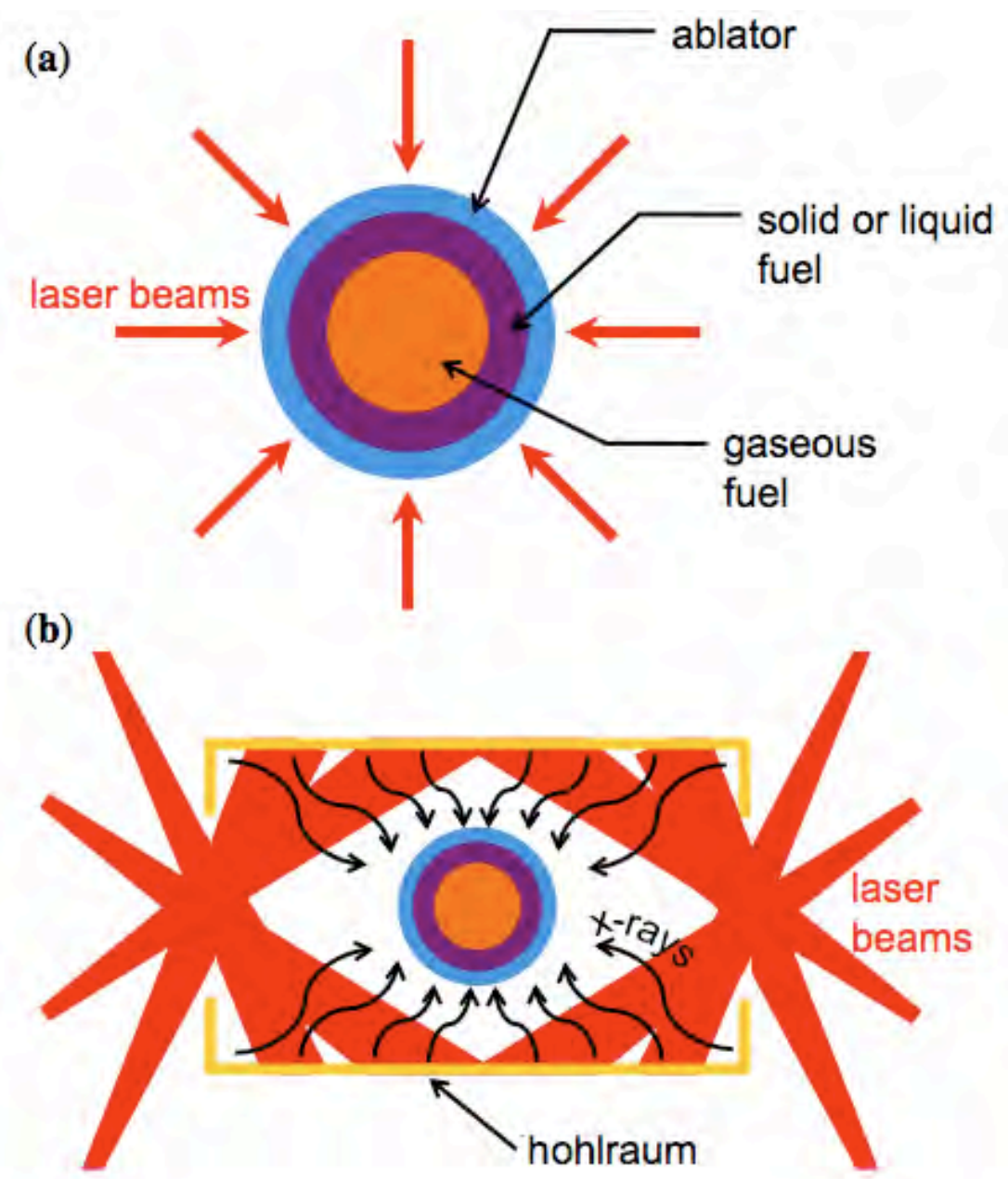

Figure 1.2: ICF uses either (a) direct drive or (b) indirect drive to deliver the laser energy to the fuel capsule.

In both the direct and indirect drive approaches, the energy from the driver would be rapidly delivered to the ablator. The ablator would then heat up and expand, and the outward momentum of the hot gas would then impart (an equal and 
opposite) inward momentum to the rest of the shell in a rocket-like reaction. The spherical capsule then begins accelerating inwards toward the center, compressing and heating the fuel via shocks and $P d V$ work. At the end of the compression, the compressed fuel will have a high temperature $\left(T_{i} \simeq 8 \mathrm{keV}\right)$ and a low density $\left(\rho R_{\text {hotspot }} \simeq 0.25 \mathrm{~g} / \mathrm{cm}^{2}\right)$ hot spot containing $2-5 \%$ of the fuel, surrounded by a low temperature, high density region comprising the remaining mass.

In the direct drive configuration, typically $\sim 2-3.5$ times more laser drive energy can be imparted to the converging shell at peak kinetic energy over indirect drive. Indirect drive is less efficient because the laser energy must first be converted into x-rays first (which can be done with efficiencies of up to $90 \%$ ), but the total laser to capsule coupling is only on the order of 10-15\%[1]. However, indirect drive allows for a smoother deposition of energy to the target because the hohlraum fills almost uniformly with x-rays. Also, x-rays provide a higher hydrodynamic efficiency since they allow for ablation at a higher density and lower exhaust velocity.

The efficiency with which the fuel can be burned up can be approximated with the formula[3],

$$
\Phi \approx \frac{\rho R_{f}}{H_{B}+\rho R_{f}}
$$

where $\rho$ is the initial mass density of the fuel, $R_{f}$ is the initial radius of the fuel capsule, and $H_{B}$ is the burn parameter,

$$
H_{B}=\frac{8 c_{s} m_{f}}{<\sigma v>}
$$

with $c_{s}$ the isothermal sound velocity $\left(c_{s}=\sqrt{2 k_{B} T / m_{f}}\right), m_{f}$ being the average mass of the fuel ions, and $\langle\sigma v\rangle$ the averaged reactivity of the target nuclei. For deuterium-tritium targets, $H_{B}$ takes on a minimum value of $\sim 6.0 \mathrm{~g} / \mathrm{cm}^{2}$ for optimal burn conditions of about $30 \mathrm{keV}$ ion temperatures [4, 5].

This implies that if we were to burn $30 \%$ of the DT fuel (a reasonable burn up fraction), from Eq. 1.2, we would require an areal density,

$$
H_{f}=\rho R_{f} \simeq 3 \mathrm{~g} / \mathrm{cm}^{2} .
$$

The mass of the spherical fuel volume can be defined as,

$$
M_{f}=\frac{4 \pi}{3} \rho R_{f}^{3}=\frac{4 \pi}{3} \frac{\left(\rho R_{f}\right)^{3}}{\rho^{2}},
$$


giving us the fuel density

$$
\rho=\sqrt{\frac{4 \pi}{3} \frac{H_{f}^{3}}{M_{f}}} \simeq \frac{300}{M_{f}^{1 / 3}} \mathrm{~g} / \mathrm{cm}^{3},
$$

where $M_{f}$ is in $\mathrm{mg}$. This means that for a $M_{f}=1 \mathrm{mg}, \rho=300 \mathrm{~g} / \mathrm{cm}^{3}$. So solid DT with a density of $\rho_{D T}=0.225 \mathrm{~g} / \mathrm{cm}^{3}$ would have to be compressed by a factor

$$
\rho / \rho_{D T}=300 / 0.225 \simeq 1500 !
$$

The energy per gram produced by DT fusion is[4]

$$
E_{D T}=17.6 \mathrm{MeV} / 5 \mathrm{AMU}=3.39 \times 10^{11} \mathrm{~J} / \mathrm{g},
$$

while the energy per gram required for heating of a DT plasma is a function of temperature[1],

$$
E_{\text {DTheating }}=0.1152 \times 10^{9} \mathrm{~T} \mathrm{J/g},
$$

where $T$ is the temperature in $\mathrm{keV}$.

For DT, $80 \%$ of the $17.6 \mathrm{MeV}$ of energy released in a reaction is associated with the neutrons, which escape the plasma without interacting. The other $20 \%$, associated with the alpha particles $\left(E_{\alpha}=3.52 \mathrm{MeV}\right)$ is deposited within the plasma and is responsible for the heating.

For thermonuclear ignition to occur, the internal heating by the alpha particles must exceed the losses due to radiation, thermal conduction, and expansion. Of these loss mechanisms, radiative losses by bremsstrahlung emission dominate. Fig. 1.3 plots the power deposited by alpha particles against the power lost by bremsstrahlung emission in a 50-50 DT plasma as a function of plasma temperature. Alpha particle heating overtakes the radiative loss at a temperature of 4.3 $\mathrm{keV}$. Therefore, temperatures of $5 \mathrm{keV}$ or higher are generally characteristic of a DT fusion plasma.

The 3.5 MeV alpha particles generated in the DT fusion reaction will move along a nearly straight path, and deposit their energy along the path length by Coulomb collisions with the electrons and ions. This range will depend on both the DT plasma temperature and the plasma density, and is plotted out in Fig. 1.4. 


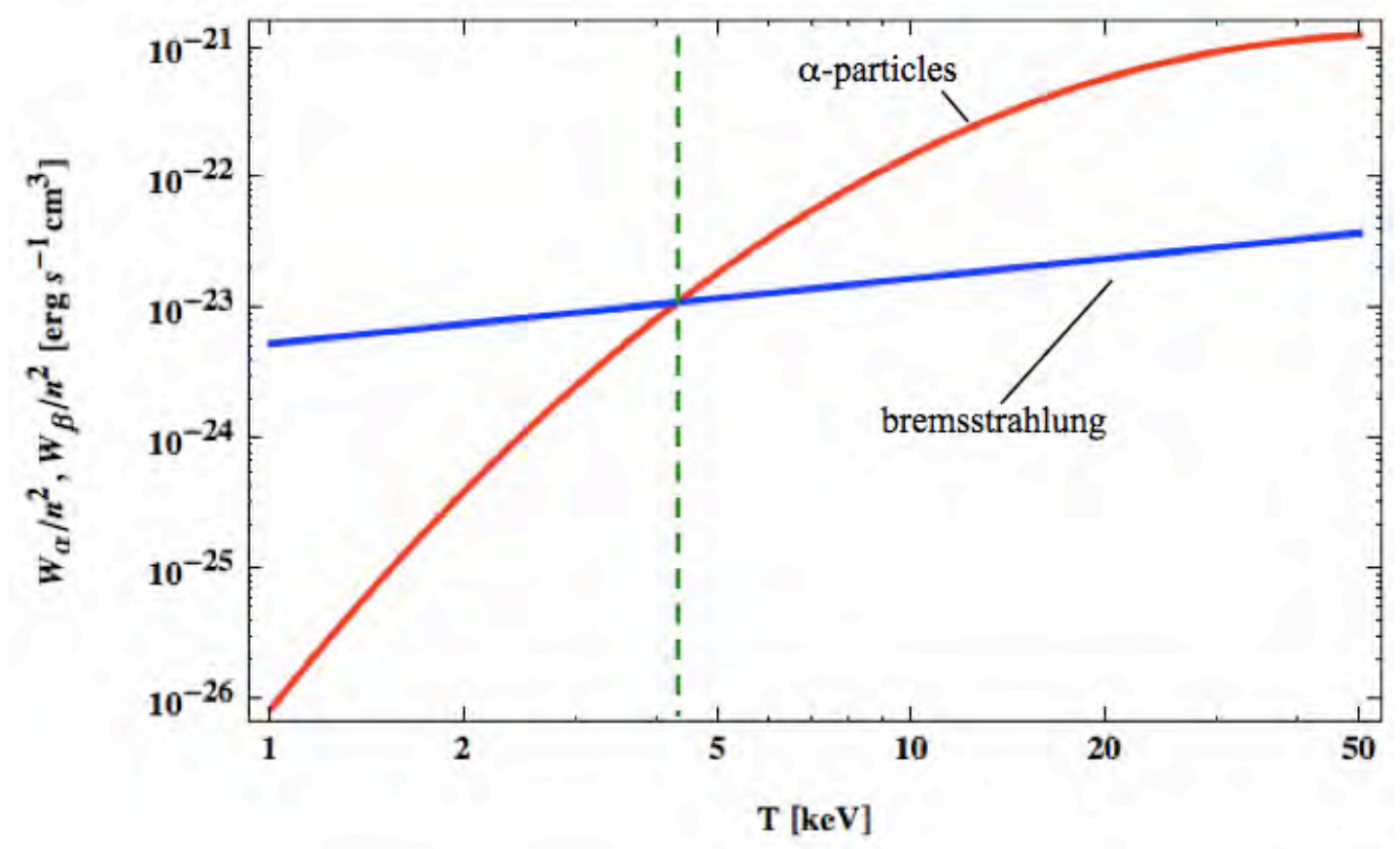

Figure 1.3: In a fusion reaction, power deposited by alpha particles is in competition with radiative losses by bremsstrahlung emission. Here power deposited by alpha particles and power emitted by bremsstrahlung in an equimolar DT plasma are plotted as a function of temperature. For ignition to occur, the DT fusion plasma must therefore exceed a temperature of $4.3 \mathrm{keV}$. Curves taken from expressions in Atzeni, The Physics of Inertial Fusion (2004)[3].

Another important quantity is the fraction of the initial alpha particle energy delivered to the ions and electrons during the entire process of slowing down, also plotted in Fig. 1.4.

At densities between $10-100 \mathrm{~g} / \mathrm{cm}^{3}$, the range of these DT alpha's can be fitted by

$$
\rho R_{\alpha}=\frac{0.025 T_{e}^{5 / 4}}{1+0.0082 T_{e}^{5 / 4}},
$$

which gives a $\rho R \simeq 0.4 \mathrm{~g} / \mathrm{cm}^{2}$ at $10 \mathrm{keV}$. Therefore, the goal is to raise the hot spot (which constitutes $\sim 2 \%$ of the total fuel mass) to a temperature of $10 \mathrm{keV}$. Deposition of the alpha particle energy then drives a burn wave that sets off an outward propagating burn. 

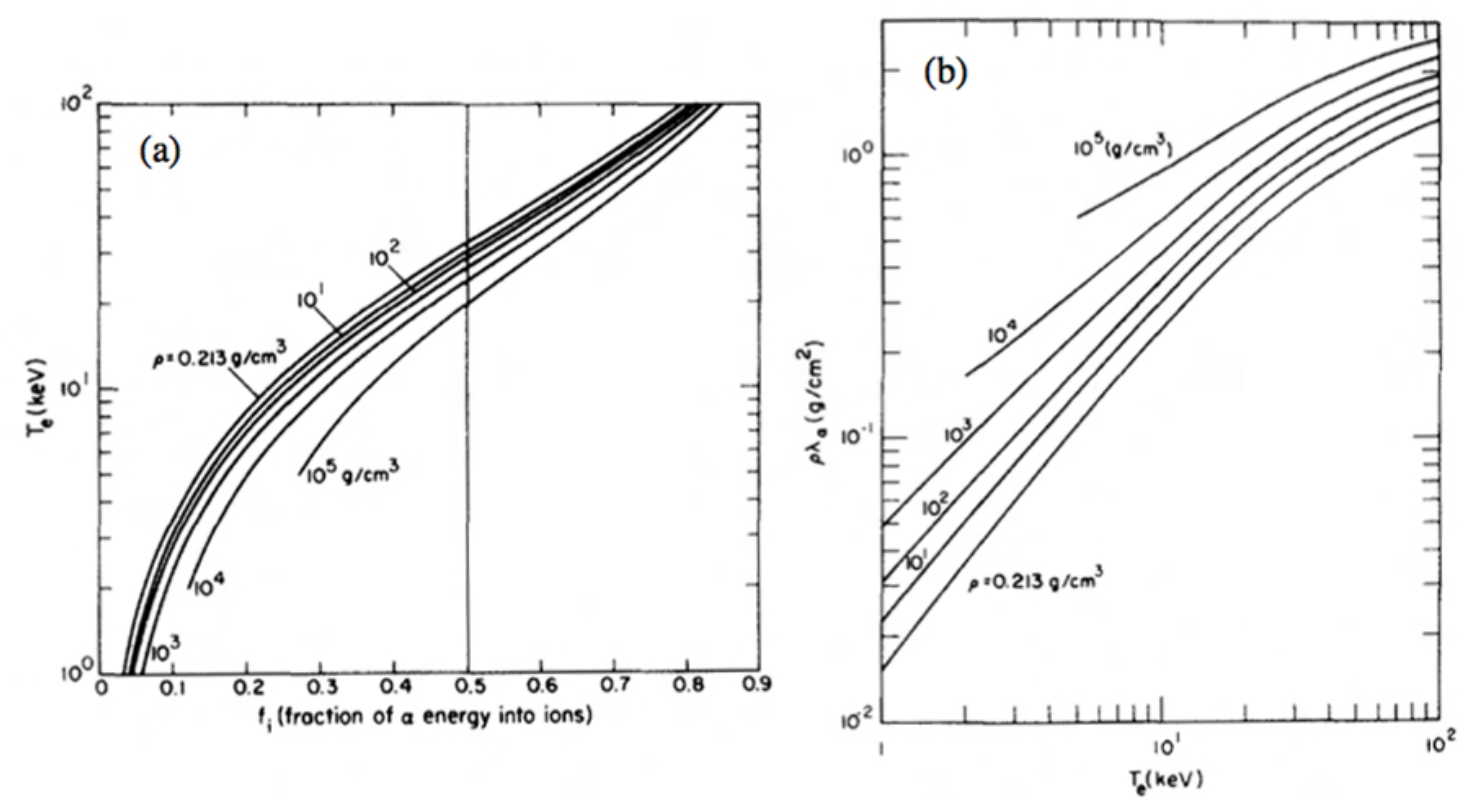

Figure 1.4: (a) Energy fraction delivered to the plasma ions for different values of the density. (b) Alpha particle stopping range versus plasma temperature for different values of the density. Figure taken from Fraley, Physics of Fluids (1974)[5].

\subsection{Motivation for Fast Ignition}

Recent advances in laser technology have allowed for petawatt $\left(10^{15} \mathrm{~W}\right)$ laser pulses to be achieved, which opens up the possibility of externally delivering energy to ignite compressed fuel. The Fast Ignition (FI) scheme[6, 7] of inertial confinement fusion (ICF) proposes to do just that - the fuel compression and ignition processes would be separated. Similar to conventional ICF, lasers would first rapidly heat the surface of the fusion target. Ablation of this material would drive a rocket-like reaction to compress the DT fuel to a high-density configuration with a hot spot. At the moment of maximum compression, a short (1-10 ps) high intensity $\left(10^{19} \mathrm{~W} / \mathrm{cm}^{2}\right)$ laser pulse would drive a small volume of the fuel to ignition. Thermonuclear burn would then spread rapidly through the compressed fuel. See Fig. 1.5. The FI approach therefore has potential advantages over conventional central hot spot ICF of reduced driver energy and reduced hydrodynamic instability. 


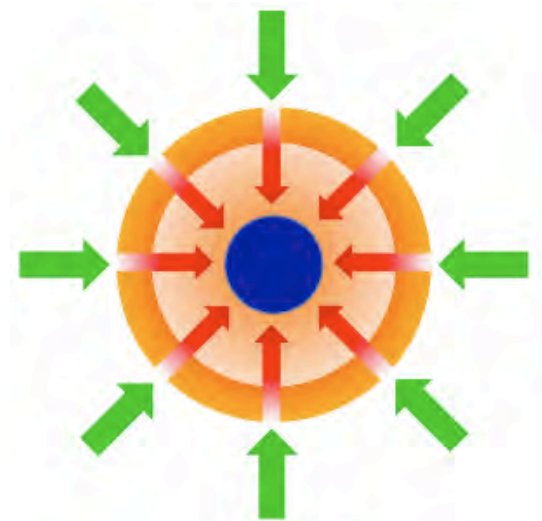

Compression

Lasers rapidly heat the surface of the fusion target. The ablation of the surface material compresses the fuel
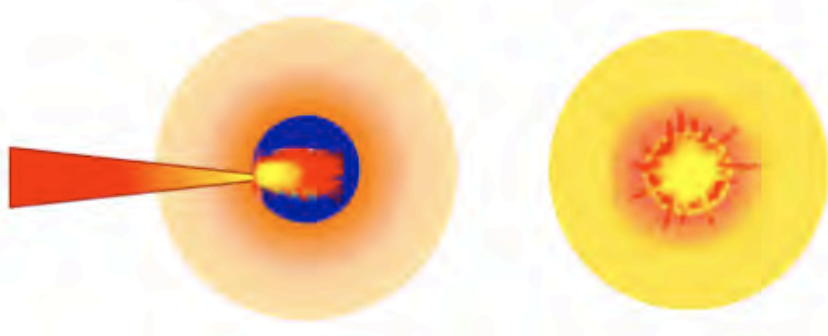

Ignition

At the moment of maximum compression, a short (1-10 ps) high intensity $\left(10^{19} \mathrm{~W} / \mathrm{cm}^{2}\right)$ pulse ignites the capsule
Burn

Thermonuclear burn spreads rapidly through the compressed fuel, yielding many times the driver input energy

Figure 1.5: A schematic of the inertial confinement fast ignition fusion concept.

As shown in Fig. 1.6, fast ignition involves main fuel assembly at isochoric (constant density) rather than isobaric (constant pressure) conditions, which allows for the advantage of a lower density, larger uniform fuel ball. Because fast ignition has less stringent requirements for fuel density, hydro issues such as mix and convergence do not play as large a role. Also, because $\rho$ is lower, there is more mass to burn $\left(E \propto M \rho^{2 / 3}\right)$ resulting in higher gain. Since target compression and hot spot formation are completely uncoupled in FI, symmetry requirements on the driver laser uniformity and target smoothness are greatly relaxed. In fact, even non-spherical fuel configurations are possible, as long as the particle beam can couple enough energy to the fuel core.

Modeling by Atzeni[8] using the 2-D code DUED, produced allowable windows for ignition in the fast ignition approach, as in Fig.??. It was found that the margins of energy, power, and intensity, as given by the lower left corners of the hatched areas, could be approximated as

$$
E_{i g n}=140\left(\frac{\rho}{100 \mathrm{~g} / \mathrm{cm}^{3}}\right)^{-1.85} \mathrm{~kJ},
$$


Conventional ICF (isobaric)

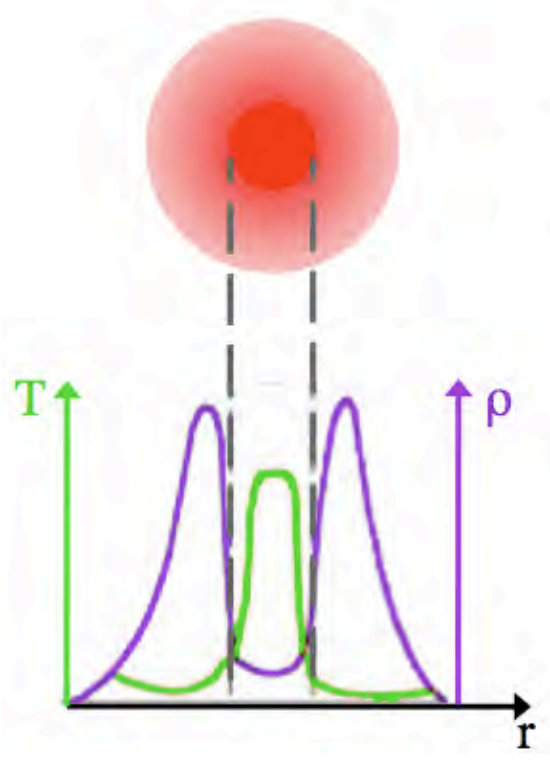

Fast Ignition (isochoric)

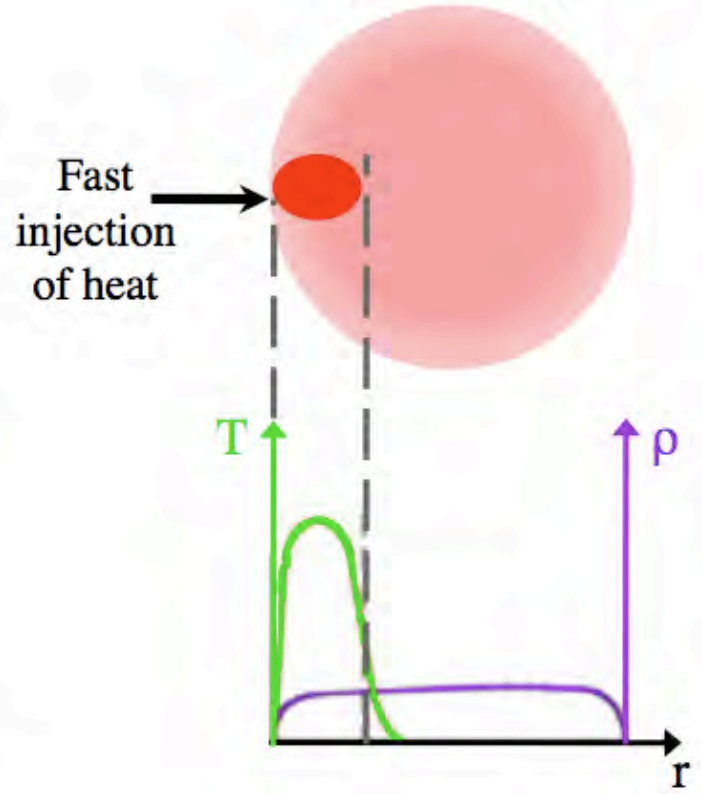

Figure 1.6: In conventional ICF, the central hot spot ignites a high density cold shell. In fast igntion, the fuel is first isochorically compressed to a moderately high density with no hot spot. Fast electrons generated from a separate high-intensity short pulse laser create a hot spot.

$$
\begin{gathered}
W_{i g n}=2.6 \times 10^{15}\left(\frac{\rho}{100 \mathrm{~g} / \mathrm{cm}^{3}}\right)^{-1} \mathrm{~W}, \\
I_{i g n}=2.4 \times 10^{19}\left(\frac{\rho}{100 \mathrm{~g} / \mathrm{cm}^{3}}\right)^{0.95} \mathrm{~W} / \mathrm{cm}^{2} .
\end{gathered}
$$

This implies that for full scale FI, the optimal set of parameters to ignite compressed DT plasma at a density of $300 \mathrm{~g} / \mathrm{cm}^{3}$ would be $\sim 6.8 \times 10^{19} \mathrm{~W} / \mathrm{cm}^{2}$, with $>18 \mathrm{~kJ}$ of the laser energy coupled into particle energy within a $20 \mathrm{ps}$ pulselength injected in a $<40 \mu \mathrm{m}$ diameter hot spot. To match the areal density $(\rho r)$ of the hot spot of $1.2 \mathrm{~g} / \mathrm{cm}^{2}$, we would require particles with a corresponding penetration depth of $1.2 \mathrm{~g} / \mathrm{cm}^{2}$. This range would be satisfied by fast electrons with an average energy of $\sim 2 \mathrm{MeV}[9]$. For this reason, fast electrons generated by the igniter laser with an energy of 1-3 MeV are of the most interest for fast ignition.

Isochoric fast ignition could lead to fuel gains 2-3 times higher than the standard 
(a)

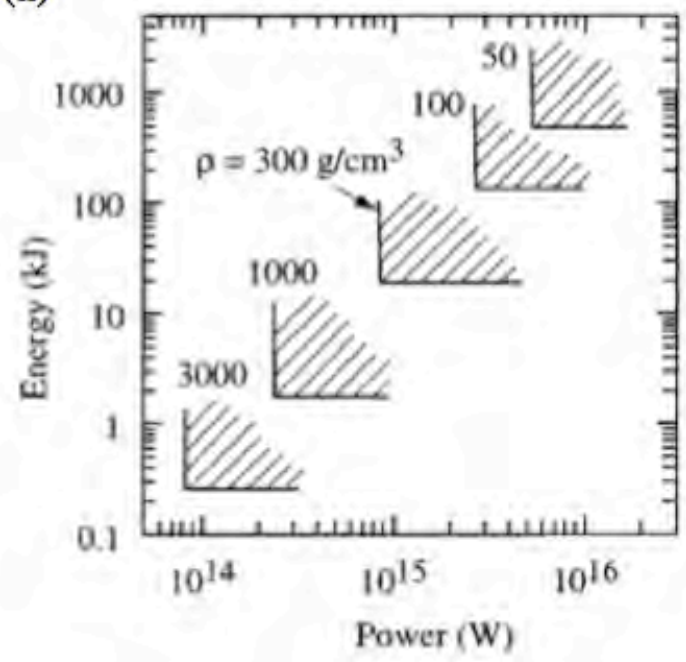

(b)

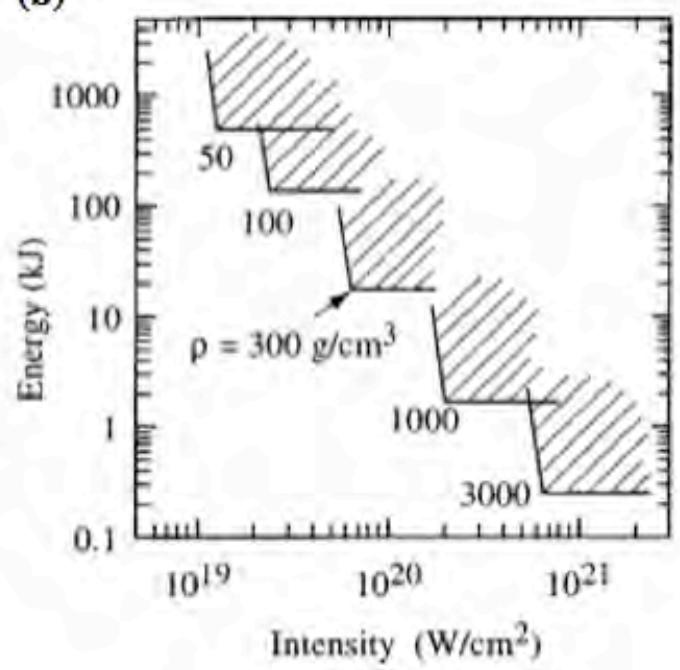

Figure 1.7: Pulse parameters for fast ignition, for particles with a range of 0.6 $\mathrm{g} / \mathrm{cm}^{2}$, as determined from 2-D DUED simulations. (a) Ignition windows in the power-energy plane, and (b) Ignition windows in the intensity-energy plane. Figure taken from Atzeni, Physics of Plasmas (1999)[8].

isobaric central hot spot ignition. Or, to think of it another way, for a given gain, the energy required to be delivered to the fuel can be about an order of magnitude less using fast ignition. This was also demonstrated by Atzeni[8] and is shown in Fig. 1.8.

These attractive features of fast ignition make it a very appealing ICF concept. In the meantime, the quest to demonstrate its viability opens up many other new categories of research in short pulse physics, target design, energy transport, scaling relations, and more.

\subsection{Cone-Guided Fast Ignition and its Require- ments}

One of the major difficulties of fast ignition is exactly how to transport a large amount of energy from the short pulse laser interaction position to the compressed fuel core. During the fuel assembly, the ablative implosion would create a signif- 


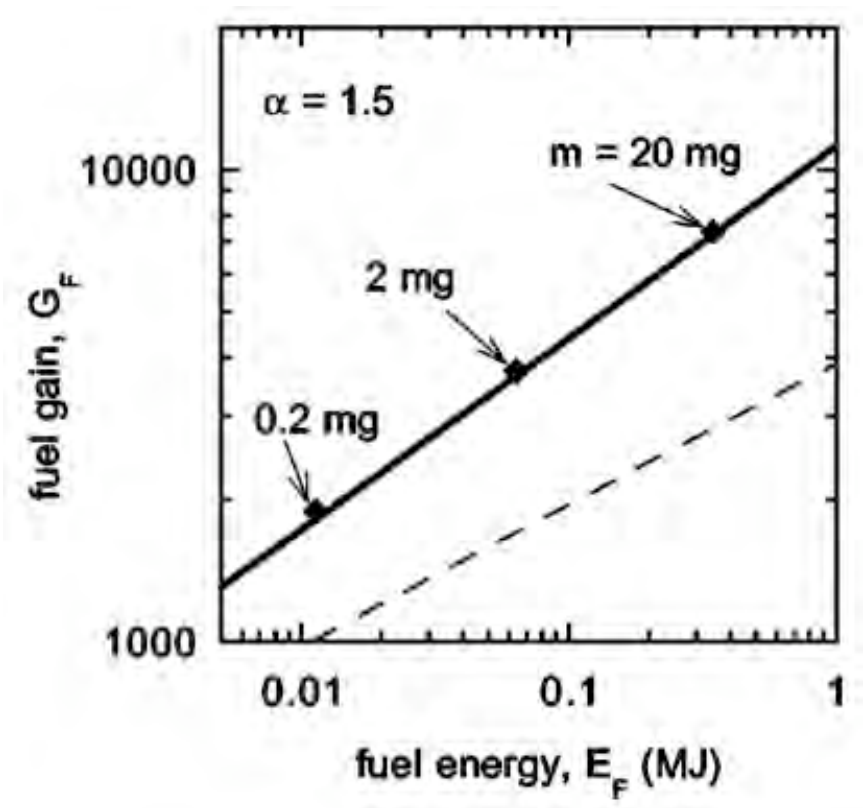

Figure 1.8: Fuel gain as a function of fuel energy. Squares : 2D numerical simulations using DUED, solid line: isochoric model (i.e., fast ignition), broken line: isobaric model (i.e., central hot spot ignition). Figure taken from Atzeni, Physics of Plasmas (1999)[8].

icant coronal plasma separating the critical surface $\left(n_{\text {crit }}\right.$, where the majority of the laser light would be absorbed) from the compressed fuel. Hot electrons created by the igniter laser would have to traverse a density gradient up to $n / n_{\text {crit }} \approx 10^{5}$ before depositing their energy. The relativistic electron beam must be composed of $>18 \mathrm{~kJ}$ of electrons with a mean energy of $2 \mathrm{MeV}$ to match the necessary deposition range, which then must travel over a distance of $\sim 100 \mu \mathrm{m}$. Obviously, finding a way to bring the laser beam closer to the fuel core would assist with each of these challenges.

One design to resolve this issue would be to use a cone embedded in the fuel capsule to provide a path free of lower density peripheral plasma produced during the implosion, as well as help guide the short pulse high-intensity laser toward the core and allow electron generation closer to the core[10, 11]. Such a capsule configuration is illustrated in Fig. 1.9. In most designs, the cone would be made of gold to have the cone material be fairly dense. Also, the high x-ray opacity of 


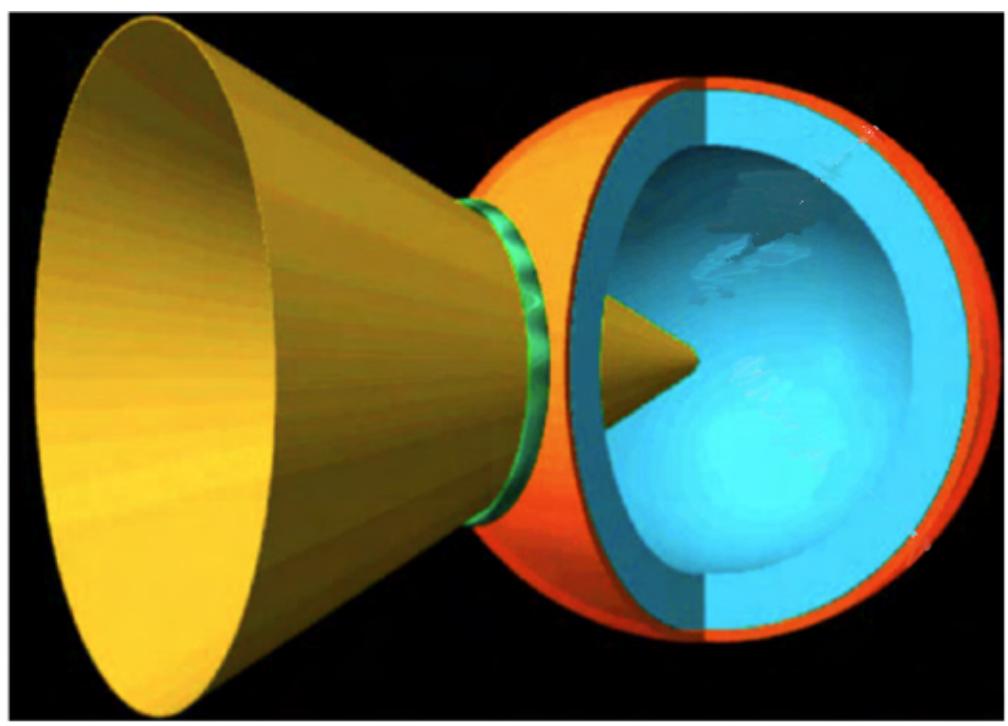

Figure 1.9: Cartoon of the cone-focus geometry for fast ignition. The cone provides an open path for the ignitor beam opposite the implosion.

gold would tend to allow the cone to be reflective of the diffuse x-rays impinging on it in the indirect drive case.

Hydrodynamics calculations were done by Hatchett et al.[12], of a spherical fuel capsule with a gold cone inserted. The fuel capsule was imploded by indirect drive $\left(\mathrm{T}_{\text {rad }}\right.$ of $\left.250 \mathrm{eV}\right)$, and it was shown that near-spherical symmetry could be achieved, with a $<\rho R>=2.2 \mathrm{~g} / \mathrm{cm}^{2}$, and a nearly unperturbed cone interior. RayleighTaylor instability was not found to be an issue at the interface between cold fuel and ignition gas with this scheme. Simulations also showed that the distance between the compressed core and the partially ablated cone tip was typically between 50 - $100 \mu \mathrm{m}$, with residual plasma in the gap. A few selected results are shown in Fig. 1.10 .

To test these theoretical predictions, Kodama et al.[13] used the Gekko XIII laser at the University of Osaka to implode a deuterated-polystyrene (CD) shell of $500 \mu \mathrm{m}$ diameter and $7 \mu \mathrm{m}$ thickness with a gold cone attached. (See Fig. 1.11a). Nine laser beams of $1 \mathrm{~ns}$ duration with a total of $1.2 \mathrm{~kJ}$ were used for the compression, while a subpicosecond short-pulse laser of $\sim 60 \mathrm{~J}$ provided the heating through the cone. A typical x-ray image of the compression without the injection 

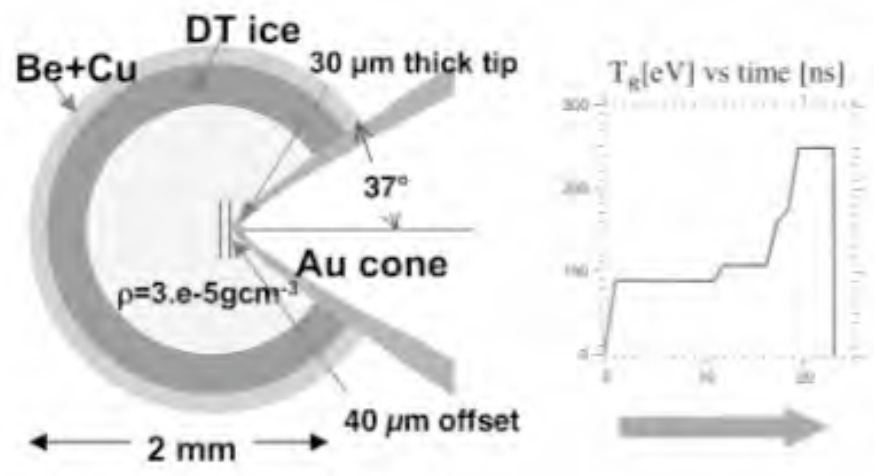

Imploded config.

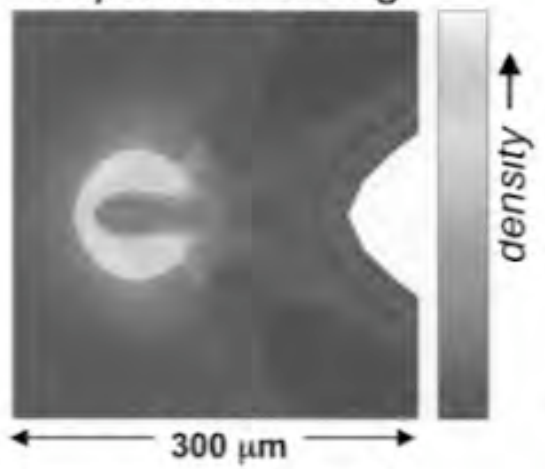

Figure 1.10: (a) The NIF ignition scale cone-guided FI baseline design. (b) A simulation with a temporal radiation profile as shown (with a $\mathrm{T}_{R, \max }$ of $250 \mathrm{eV}$ ), where the capsule absorbs $\sim 150 \mathrm{~kJ}$ of x-ray energy results in (c) the imploded configuration with a solid-angle-averaged fuel column density $\left\langle\rho \mathrm{R}>_{D T}=2.2 \mathrm{~g} / \mathrm{cm}^{2}\right.$. Figure taken from Hatchett, Fusion Science Technology (2006)[12].

of the short pulse laser is shown in Fig. 1.11b. The imploded core plasma, found to be of a density $0.1-0.5 \mathrm{~g} / \mathrm{cm}^{3}$ and a diameter of $40-45 \mu \mathrm{m}$, can be seen close to the tip of the cone. Hot electrons created by the short-pulse were found to have a $k T$ of $2-3 \mathrm{MeV}$, and were created with a conversion efficiency of $30-40 \%$. Neutrons created by the thermonuclear D-D reaction were 10 times more plentiful when the heating pulse was timed to coincide with maximum compression than when no heating pulse was present. This corresponded to a total energy required of about half what would have been necessary to achieve similar neutron yields with no heating pulse and a spherically symmetric implosion.

While Kodama et al. was able to demonstrate efficient simultaneous compression and heating in a cone-guided FI geometry as a feasible approach to ICF, the experiment still needs to be scaled up to temperatures and densities that would actually ignite a DT pellet. Actual conditions will be far more stressful, with greater core densities, larger electron fluxes, and strict laser requirements. Many critical physics issues confronting the fast ignition scheme still remain.

Some of these key issues are shown in Fig. 1.12. To optimize the implosion to produce the cold dense fuel core, the fuel capsule and the laser drive must be designed to minimize hot spot and meet the $\rho$ and $\rho R$ requirements. In conjunction, 


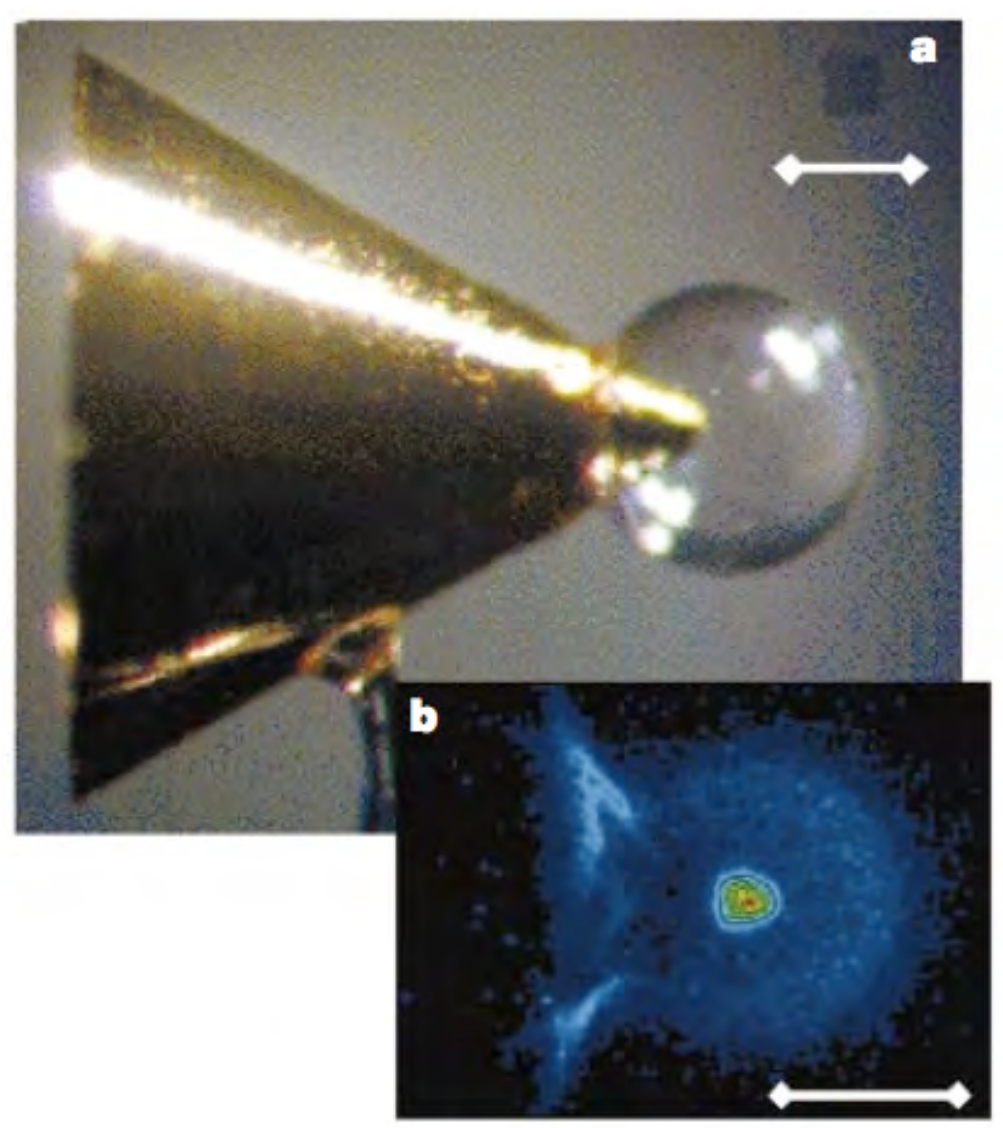

Figure 1.11: (a) The reentrant cone target used by Kodama et al. The gold cone was attached to a CD shell of $500 \mu \mathrm{m}$ diameter and $7 \mu \mathrm{m}$ wall thickness. (b) An x-ray image showing the imploded core plasma at the cone tip. Figure taken from Kodama, Nature 2001[13].

this fuel assembly must be achieved in a hydrodynamics configuration that allows the cone to survive and not collapse on itself due to the high pressures.

A high conversion efficiency from laser to useful hot electrons to then ignite the fuel is one of the most important criterion. This is partially dependent on the incident profile of the short pulse laser, which must be selected carefully since the intensity, focus, and pointing will affect the number and energy of electrons produced. One concern is the contrast level of the laser, and how much preplasma the laser pedestal will create in the cone which could hinder the main pulse from reaching the cone tip. The preplasma may cause filamentation or self-focusing of the laser beam and will push the critical surface away from the cone tip, in- 
herently changing the properties of the electrons produced. Once those electrons are produced, how they transport through the cone (and whether they reflux or get trapped or diverge) and their propagation and stopping in the fuel are major questions.

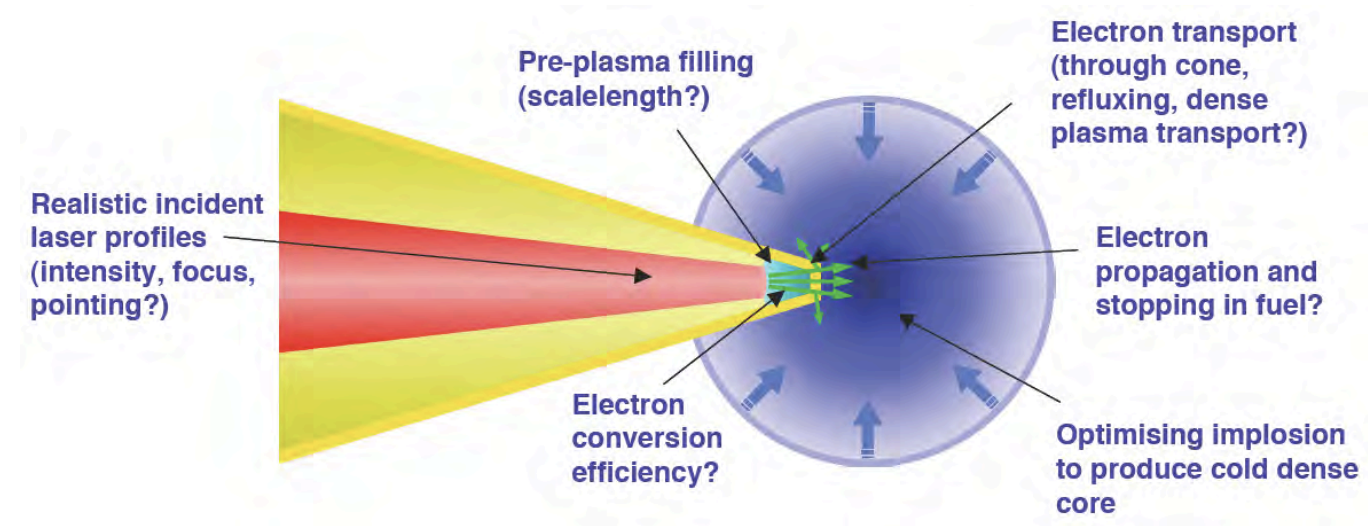

Figure 1.12: The key issues for electron cone-guided fast ignition include tailoring the incident laser profile, understanding the effect of preplasma, optimizing the electron conversion efficiency, characterizing the electron transport, and determining the propagation and stopping of the electrons in the fuel.

The study of the generation and transport of fast electrons is fundamental to understanding high-intensity laser-solid interactions because they are a major factor in the absorption and transport of the laser energy. Therefore, in this thesis, we attempt to address some of these issues.

\subsection{Outline of the Dissertation}

Chapter Two provides an introduction to some of the basics of ultra-intense laser-plasma interactions and some of the absorption mechanisms that allow energy to be transferred from the laser to the plasma. In addition, background will be provided on the generation and transport of relativistic electron beams in solid targets.

Chapter Three gives an overview of the Titan and Vulcan laser facilities where the experiments described in this thesis were undertaken. A few of the basic tech- 
nologies that make short-pulse high-intensity lasers possible (such as Chirped Pulse Amplification and mode-locking) are discussed, as well as some of the drawbacks (such as prepulse). The emphasis, of course, is on the accurate diagnosis of the laser-plasma interaction, so this chapter delves into both the methods of characterizing the laser and the detection of target self-emission. A number of the key diagnostic instruments such as the XUV imager, the $\mathrm{Cu} \mathrm{K} \alpha$ imager, the HOPG spectrometer, and the Single Photon Counting Camera are introduced.

Chapter Four describes a diagnostic technique involving extreme ultraviolet (XUV) imaging at two different energies $(256 \mathrm{eV}$ and $68 \mathrm{eV})$ to measure the plasma temperature at the rear surface of foil targets. Spatially resolved rear surface temperature patterns were determined by comparing absolute intensities to LASNEX radiation hydrodynamic modeling. Results from the calibration of the multilayer XUV mirrors at the Advanced Light Source at the Lawrence Berkeley Laboratory are also presented. Temperatures derived from the imagers are then also validated against temperatures measured using XUV spectroscopy with a flat-field spectrometer.

Chapter Five discusses an experiment in which nail-wire targets were irradiated using the Vulcan Petawatt Laser to investigate energy transport by relativistic fast electrons. By using several $\mathrm{Cu} \mathrm{K} \alpha$ diagnostics, it was shown that the energy deposition was concentrated in the nail head, with an approximately exponential fall-off along the wire with a short decay length due to resistive inhibition. Also, XUV imaging indicated the heating of a thin surface layer of the wire. e-PLAS implicit-moment/hybrid simulations completed in conjunction showed qualitative agreement with the experimental data.

Chapter Six reports the results of a detailed study to measure conversion efficiency and $\mathrm{T}_{\text {hot }}$ of hot electrons generated in cone-wire targets irradiated with the Titan short pulse laser at varying prepulse levels. Using the HYDRA radiationhydrodynamics code, the distribution of preformed plasma inside the cone was modeled, and then the Zuma hybrid transport code was used to infer the initial electron source distribution entering the wire from the observed $\mathrm{K} \alpha$ signal in the wire. While coupling was found to decrease by approximately a factor of 8 in 
increasing the prepulse energy from $8 \mathrm{~mJ}$ to $1000 \mathrm{~mJ}$, the temperature of the hot electrons was not found to vary significantly.

Chapter Seven summarizes the conclusions and proposes future experiments and modeling to expand upon the work discussed herein.

\subsection{Role of the author}

High-intensity short pulse laser experiments on a major facility necessarily involve a large collaborative team effort. This section outlines the specific role of the author in the work described in this thesis.

The experimental data in Chapter 4 were drawn from several experimental campaigns on the Titan Laser at LLNL in April 2007, August 2007, and September 2007. The author was responsible for target area setup, beamline optimization (laser alignment, focusing, and focal spot imaging), setting up and optimizing the XUV imager and XUV flat field spectrometer diagnostics, and collecting the data from them. The LASNEX simulations used to translate XUV images into temperatures were completed by Steve Hatchett. The calibration of the XUV mirrors was completed at the Lawrence Berkeley National Laboratory by the author and Andrew MacPhee, with the assistance of Franklin Dollar and Eric Gullikson, who provided us with beam time and ran the Optics beamline 6.3.2 at the Advanced Light Source. The FLYCHK simulations to generate synthetic emission spectra for comparison against the experimental spectra were completed solely by the author. All analysis was performed by the author.

The nail-wire experiment described in Chapter 5 was performed on the Vulcan Petawatt Laser in the U.K. in the summer of 2006 by the author. Setup of the $\mathrm{Cu} \mathrm{K} \alpha$ imager and HOPG diagnostics, and collection of the data from them was done by the author. The analysis technique applied to that set of data was largely developed by James King and Mike Key to analyze the cone-wires in the 2009 paper by King et al.[14] The 1-D transport code used to fit the data in Chapter 5 was developed and carried out by Kramer Akli. The e-PLAS simulations were kindly completed by Rod Mason of Research Applications Corporation. All analysis of 
the experimental data and interpretation of the modeling was done by the author.

In Chapter 6, the experimental data was collected on the Titan Laser Facility in August of 2009. The author was largely involved in the planning, setup, and execution of the experiment. Target design and specifications, as well as laser shot parameters were completely managed by the author. During the experiment, the author was responsible for laser alignment, focusing, and pointing, and imaging of the focal spot. All data analysis, HYDRA and Zuma modeling, and post-processing were done solely by the author. 


\section{Chapter 2}

\section{Physics of Intense Laser Plasma Interactions}

\section{$2.1 \quad$ Introduction}

When an intense laser irradiates a solid target, a plasma rapidly forms on the front surface. This plasma, itself still evolving, will thereafter have a large effect on the propagation of the laser light. Within the plasma, the light wave will propagate with the dispersion relation

$$
k^{2} c^{2}=w_{0}^{2}-w_{p e}^{2},
$$

where $k$ is the wavenumber of the laser light, $c$ is the speed of light, $\omega_{0}$ is the laser frequency, and $w_{p e}=\sqrt{\frac{4 \pi n_{e} e^{2}}{m_{e}}}$ is the plasma frequency.

As the laser continues traveling into the plasma, its wave vector becomes smaller until it encounters the critical density of the plasma, and can no longer propagate. The critical density is defined as

$$
n_{\text {crit }} \equiv \frac{m_{e} \omega_{0}^{2}}{4 \pi e^{2}},
$$

or in more practical units,

$$
n_{\text {crit }} \simeq 1.1 \times 10^{21}\left(\frac{\lambda}{\mu \mathrm{m}}\right) \mathrm{cm}^{-3} .
$$

For the case of a Nd:glass laser where $\lambda \sim 1054 \mathrm{~nm}, n_{\text {crit }}$ occurs at approximately $1 \times 10^{21} \mathrm{~cm}^{-3}$. 
Beyond the critical density, the wave vector becomes imaginary, and the wave is exponentially attenuated within a skin depth, defined as

$$
\delta=[\operatorname{Im}(k)]^{-1}=\frac{c}{\left(\omega_{p e}^{2}-\omega_{0}^{2}\right)^{1 / 2}} .
$$

Because the laser light cannot propagate in densities beyond $n_{\text {crit }}$, nearly all laser light absorption and reflection processes occur in the "underdense plasma," where $n_{e}<n_{\text {crit }}$. While the light may be scattered or distorted as it propagates through the underdense plasma, the majority of the light will be absorbed at or near the critical density. The thermal energy deposited in the plasma by the laser will then be conducted toward the colder, denser regions beyond the critical density, or "overdense plasma," by electron thermal conduction.

Energy is transferred to the electrons through the oscillating electric field of the laser. The EM wave will oscillate the electrons transversely along the direction of the electric field vector with a quiver velocity, $v_{\text {osc }}$, which is more generally defined by the normalized momentum,

$$
a_{0}=\frac{p_{o s c}}{m_{e} c}=\frac{\gamma v_{o s c}}{c}=\frac{e E_{0}}{m_{e} c \omega_{0}}=\sqrt{\frac{I \lambda_{\mu m}^{2}}{1.37 \times 10^{18}}},
$$

where $p_{\text {osc }}$ is the transverse quiver momentum.

For values of $a_{0}<1$, which occurs when $I \lambda^{2}<10^{18}\left(\mathrm{~W} / \mathrm{cm}^{2}\right) \cdot \mu \mathrm{m}^{2}$ (for $1 \mu \mathrm{m}$ light), the ponderomotive force of the laser accelerates electrons in the perpendicular direction over many microns. When $a_{0}>1$, the laser magnetic field becomes non-negligible, and electrons can be accelerated longitudinally by the longitudinal component of the $-e \mathbf{v} \times \mathbf{B}$ (Lorentz force). An $a_{0}>4$ corresponding to an $I \lambda^{2}$ of roughly $2.2 \times 10^{19}\left(\mathrm{~W} / \mathrm{cm}^{2}\right) \cdot \mu \mathrm{m}^{2}$ is considered the ultrarelativistic regime.

For the laser intensities we are concerned with in this thesis, the light is often intense enough to accelerate the electrons to relativistic energies. The enhanced mass of the electrons, $\gamma m_{e}$, will allow the light to propagate to a relativistic critical surface, $\gamma n_{\text {crit }}$, where $\gamma$ is the relativistic factor, $\gamma=\sqrt{\left(1+a_{0}^{2}\right)}$ for circularly polarized light and $\gamma=\sqrt{1+a_{0}^{2} / 2}$ for linearly polarized light.

As the laser pulse is incident on the target, a plasma is very rapidly formed by the early part of the light pulse ionizing the target. This underdense plasma 
that is created will have some density gradient leading up to the critical surface. The subsequent laser light is then initially incident on this lower density plasma rather than the solid target. In cases where a laser may have a significant prepulse, (some non-negligible pedestal of laser energy preceding the main pulse), a fairly long scalelength of plasma can form. The plasma scalelength is defined as

$$
L=\left(\frac{1}{n_{e}} \frac{d n_{e}}{d x}\right)^{-1}
$$

or $L=c_{s} \tau$, where $c_{s}$ is the sound speed

$$
c_{s}=\sqrt{k_{B} \frac{Z T_{e}+T_{i}}{M_{i}}} .
$$

The length of this plasma density scalelength will have a significant influence on the laser absorption and fast electron temperature, as it will often determine which absorption mechanisms dominate. The characteristics of the plasma density profile when the main laser pulse is incident will have a significant influence on the hot electron number and distribution that is generated. The steeper the density profile, the smaller the skin depth, and the less penetrating the electromagnetic fields of the laser will be, therefore leading to fewer and less energetic fast electrons.

The physics mechanisms of high intensity laser propagation in underdense plasma, as well as absorption in the overdense plasma will be discussed in this chapter.

\subsection{Ponderomotive Force}

The weak radiation pressure of light waves can become significant in the regime of intense lasers, and when applied to a plasma can become a strong nonlinear force known as the ponderomotive force.

A tightly focused short pulse laser will create strong radial light pressure intensity gradients that will act to push electrons away from the regions of high laser intensity. The ponderomotive force can thus be expressed as the gradient of the energy of oscillation in the longitudinal and transverse fields [15]. 
Begin with the non-relativistic case of a single electron oscillating near the center of a focused beam. In the limit $v / c<<1$, the electron equation of motion is

$$
m \frac{d \mathbf{v}}{d t}=-e\left[\mathbf{E}(\mathbf{r})+\frac{\mathbf{v} \times \mathbf{B}(\mathbf{r})}{c}\right] .
$$

Assume the wave has an electric field of the form

$$
\mathbf{E}=\mathbf{E}_{\mathbf{0}}(\mathbf{r}) \cos \left(\omega_{0} t\right),
$$

where $\mathbf{E}_{\mathbf{0}}(\mathbf{r})$ has a spatial dependence. We will then write the electron velocity as a sum of first and second order terms, $\mathbf{v}=\mathbf{v}_{\mathbf{1}}+\mathbf{v}_{\mathbf{2}}$. If we were to consider just the instantaneous, equilibrium position of an electron, then the $\mathbf{v} \times \mathbf{B}$ term would vanish. However, the nonlinearity comes into play when we evaluate the $\mathbf{E}$ at the actual position of the particle, and in this case the $\mathbf{v} \times \mathbf{B}$ force will have a non-negligible effect on the orbit of the electron.

Starting in first order at the initial position $\mathbf{r}_{0}$, the $\mathbf{v} \times \mathbf{B}$ can be neglected, and

$$
\begin{gathered}
m \frac{d \mathbf{v}_{\mathbf{1}}}{d t}=-e \mathbf{E}\left(\mathbf{r}_{\mathbf{0}}\right) \\
\mathbf{v}_{\mathbf{1}}=-\frac{e}{m \omega} \mathbf{E}_{\mathbf{0}}\left(\mathbf{r}_{\mathbf{0}}\right) \sin \left(\omega_{0} t\right)=\frac{d \mathbf{r}_{\mathbf{1}}}{d t} \\
\mathbf{r}_{\mathbf{1}}=\frac{e}{m \omega_{0}^{2}} \mathbf{E}_{\mathbf{0}}\left(\mathbf{r}_{\mathbf{0}}\right) \cos \left(\omega_{0} t\right) .
\end{gathered}
$$

Now moving onto second order, Taylor expansion of the electric field around the initial position of the electrons gives

$$
\mathbf{E}(\mathbf{r})=\mathbf{E}\left(\mathbf{r}_{\mathbf{0}}\right)+\left.\left(\mathbf{r}_{\mathbf{1}} \cdot \nabla\right) \mathbf{E}\right|_{\mathbf{r}=\mathbf{r}_{\mathbf{0}}}+\cdots
$$

At this point, the $\mathbf{v} \times \mathbf{B}$ term reenters the equation, and the motion of the electrons in second order is

$$
m \frac{d \mathbf{v}_{\mathbf{2}}}{d t}=-e\left[\left(\mathbf{r}_{\mathbf{1}} \cdot \nabla\right) \mathbf{E}+\frac{\mathbf{v}_{\mathbf{1}} \times \mathbf{B}_{\mathbf{1}}}{} c\right] .
$$

Integrating Faraday's law, $\partial \mathbf{B} / \partial t=-c \nabla \times \mathbf{E}$, gives the magnetic field, $\mathbf{B}_{1}$ :

$$
\mathbf{B}_{\mathbf{1}}=-\frac{c}{\omega_{0}} \nabla \times\left.\mathbf{E}_{\mathbf{0}}\right|_{r=r_{0}} \sin \left(\omega_{0} t\right) .
$$


Substituting our expressions for $\mathbf{v}_{1}, \mathbf{B}_{1}$, and $\mathbf{r}_{1}$ into Eq. 2.14 and averaging over the laser period, gives

$$
\begin{aligned}
\mathbf{F}_{\mathbf{p}}=m\left\langle\frac{d \mathbf{v}_{\mathbf{2}}}{d t}\right\rangle & =-\frac{1}{2} \frac{e^{2}}{m \omega_{0}^{2}}\left[\left(\mathbf{E}_{\mathbf{0}} \cdot \nabla\right) \mathbf{E}_{\mathbf{0}}+\mathbf{E}_{\mathbf{0}} \times\left(\nabla \times \mathbf{E}_{\mathbf{0}}\right)\right] \\
& =-\frac{1}{2} \frac{e^{2}}{m \omega_{0}^{2}} \nabla<\mathbf{E}_{\mathbf{0}}^{2}(\mathbf{r})> \\
& =-\frac{e^{2}}{4 m \omega_{0}^{2}} \nabla \mathbf{E}_{\mathbf{0}}{ }^{2}
\end{aligned}
$$

This is the nonlinear ponderomotive force that distorts the electron orbits.

The ponderomotive force can also be written as a force per unit volume by multiplying by the electron density $n_{0}$. Then,

$$
\mathbf{F}_{\mathbf{p}, \mathbf{v o l}}=-\frac{\omega_{p}^{2}}{\omega_{0}} \nabla \frac{<\epsilon_{0} \mathbf{E}^{2}>}{2}
$$

showing that the ponderomotive force is proportional to the plasma density and the gradient of the laser intensity $\left(\mathbf{I} \propto \mathbf{E}^{2}\right)$.

\subsection{Laser Interaction with Underdense Plasma}

Because high intensity lasers invariably have a pre-pedestal of energy that creates a preformed plasma, the main laser pulse will often have to propagate through a region of underdense plasma $\left(n_{e}<n_{\text {crit }}\right)$ before reaching the critical surface where its energy is primarily absorbed. Depending on the properties of the laser itself, the beam can be distorted or modified, and even transfer some fraction of its energy to the underdense plasma.

\subsubsection{Self-Focusing and Filamentation}

In reality, the wavefront of the laser beam will be nonuniform, with regions of varying intensities. As this laser propagates through the plasma toward the critical surface, the plasma can act to magnify those intensity modulations, giving rise to self-focusing (if it occurs on the macro-scale of the full laser beam), or filamentation (if it only involves a small portion of the beam). 


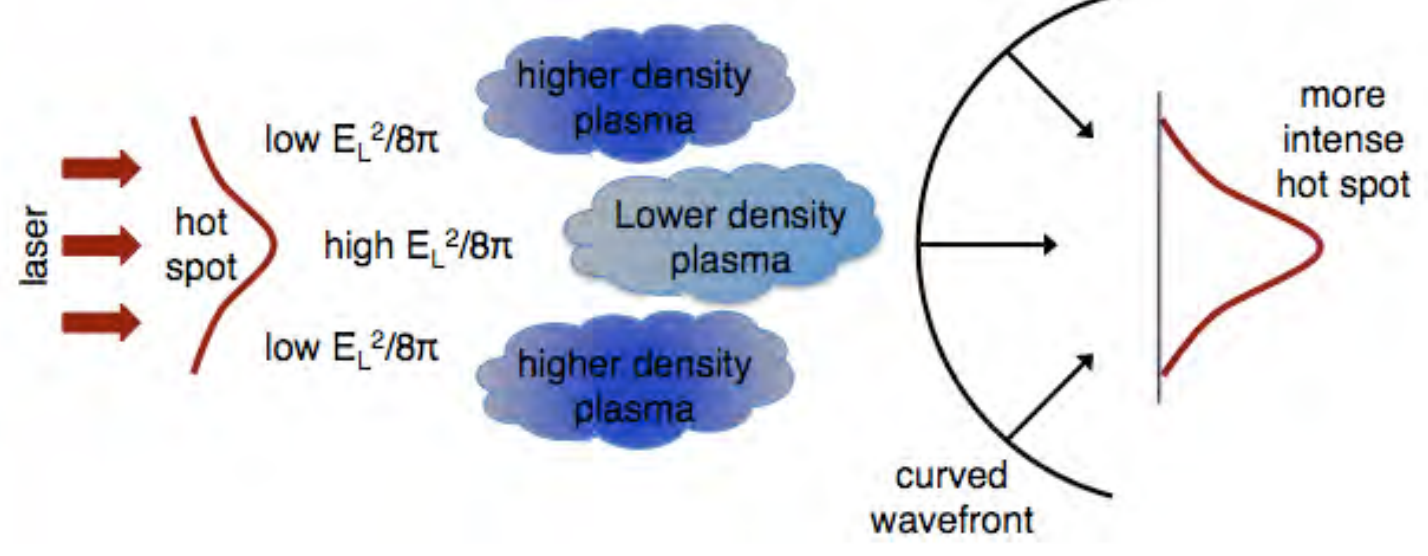

Figure 2.1: The mechanism behind self-focusing and filamentation caused by the ponderomotive force.

In a region of higher intensity, the light pressure, proportional to $\mathbf{E}^{2} / 8 \pi$, will be greater, and therefore push the plasma aside. The consequently lower plasma density in that region means that the refractive index of the plasma,

$$
\mathrm{n}=\frac{k_{L} c}{\omega_{L}}=\left(1-\frac{n_{e}}{n_{\text {crit }}}\right)^{1 / 2}
$$

will correspondingly increase. This causes a bending in the wavefront of the laser, and since the energy flux of the laser travels normal to the wavefront, the curvature produces a focusing effect which increases the intensity even further.

Relativistic self-focusing is a similar phenomenon, where at high enough laser intensity, relativistic self-focusing of the laser beam can occur due to the change of the refractive index of the plasma at relativistic quiver motion. The refractive index is peaked at the center, so the phase velocity of wave fronts passing through the focusing medium will travel more slowly at the center than at the edge.

These are all processes of positive feedback on any intensity fluctuation and are thus unstable. Once it forms, a filament can be subject to bending perturbations, scattering, diffraction, or refraction in the plasma, and can break up. In some cases, the filament manages to remain intact long enough to propagate to the critical density where it can generate hot electrons with a hot electron temperature $T_{h o t}$ 
proportional to the local laser intensity. Hot electron production and $T_{\text {hot }}$ scaling will be discussed in more detail in a later section in this chapter.

\subsubsection{Inverse Bremsstrahlung Absorption}

Inverse bremsstrahlung is the collisional absorption of energy when an electron passing through the field of an ion absorbs radiation, raising the energy and temperature of the electron particle[16, 17]. The electron particles, which were previously simply oscillating back and forth in the laser electric field, now experience momentum-changing collisions. Thus, the laser light effectively gets damped as energy is transferred to these electrons, and the plasma proportionally heats up.

Because inverse bremsstrahlung is due to these electron-ion collisions, it will scale as the electron-ion collision frequency, which can be estimated as such: The collision frequency is $\nu_{e i} \simeq n_{i} \sigma v_{t e}$, where $v_{t e}$ is the thermal velocity of electrons colliding with background ions of number density $n_{i}$. The collision cross section is $\sigma \simeq \pi b^{2}$, where $b$ is the distance of closest approach. At this distance, the potential and kinetic energies will be balanced, i.e.,

$$
\frac{Z e^{2}}{b}=\frac{1}{2} m_{e} v_{t e}^{2}
$$

Solving for $b$ and substituting into our equations above, we find

$$
\nu_{e i} \simeq 4 \pi \frac{e^{4}}{m_{e}^{2}} \frac{n_{i} Z^{2}}{v_{t e}^{3}} \propto \frac{n_{i} Z^{2}}{T_{e}^{3 / 2}} \propto \frac{n_{e} Z^{2}}{T_{e}^{3 / 2}}
$$

(because for a Maxwellian distribution of electrons, $v_{t e}$ is $\sim T_{e} m$ ). Thus we find that inverse bremsstrahlung is strongest for low temperatures, high densities, and for high-Z materials.

In fact, many other factors also contribute to the efficiency of absorption through inverse bremsstrahlung. Kruer [16] shows that a steep density profile near the critical density surface can strongly diminish the absorption. Also, at high laser intensities, the electron-ion collision frequency is altered by the oscillatory motion of the electrons in the laser electromagnetic fields. Then, the simple

derivation done above, which scales as $v_{t e}$ above is modified by a factor $\frac{e E_{L}}{m_{e} \omega_{0}}$, which results in decreasing the absorption. Finally, inverse bremsstrahlung absorption 
is most effective with electrons with a $\nu_{e i}$ close to the laser frequency. When the electron distribution is far from Maxwellian, as in cases when the the high laser intensity transfers energy to the electrons far faster than the distribution can thermalize, then there will be a large number of electrons with $v_{t e} \nu_{e i}>>\omega_{0}$ which are not strongly perturbed by collisions with the ions and which will not participate in the inverse bremsstrahlung transfer of energy. Therefore, we expect inverse bremsstrahlung to be a dominant absorption means for long laser pulses impinging on a sizable underdense plasma, but not in a short pulse interaction.

\subsection{Laser Interaction with Overdense Plasma}

In the neighborhood of the critical density, a number of laser absorption mechanisms transfer momentum from the laser electric field to the electrons. Here, resonance absorption, vacuum heating, and $\mathbf{j} \times \mathbf{B}$ heating are described.

\subsubsection{Resonance Absorption}

Resonant plasma oscillations can be excited at the critical density surface when $p$-polarized light is obliquely incident on a plasma density gradient[18, 19]. Figure 2.2 illustrates this process in detail. For laser light that is incident on the plasma at some angle $\theta$ to the normal, by Snell's law and the dispersion relation, the light will propagate up the density $n_{e}(\theta)=n_{\text {crit }} \cos ^{2} \theta$ before it is specularly reflected. This density is called the classical turning-point for the light wave. As long as the light is $p$-polarized, a component of the electric field vector will point in the direction of the density gradient at the turning-point, i.e. $\mathbf{E} \cdot \nabla n_{e} \neq 0$. The electric field can then tunnel through to the critical density, where it will oscillate electrons along that plane, resonantly driving Langmuir (electron-plasma) waves.

However, in the case of $s$-polarized light, when the electric field vector is perpendicular to the plane of incidence, $\left(\mathbf{E} \cdot \nabla n_{e}=0\right)$, at the turning point of the light wave, there is no component of the electric field that will be capable of tunneling in and driving Langmuir waves.

Ginzberg[18], Forslund[20], and Freidberg[19] determined that a maximum ab- 


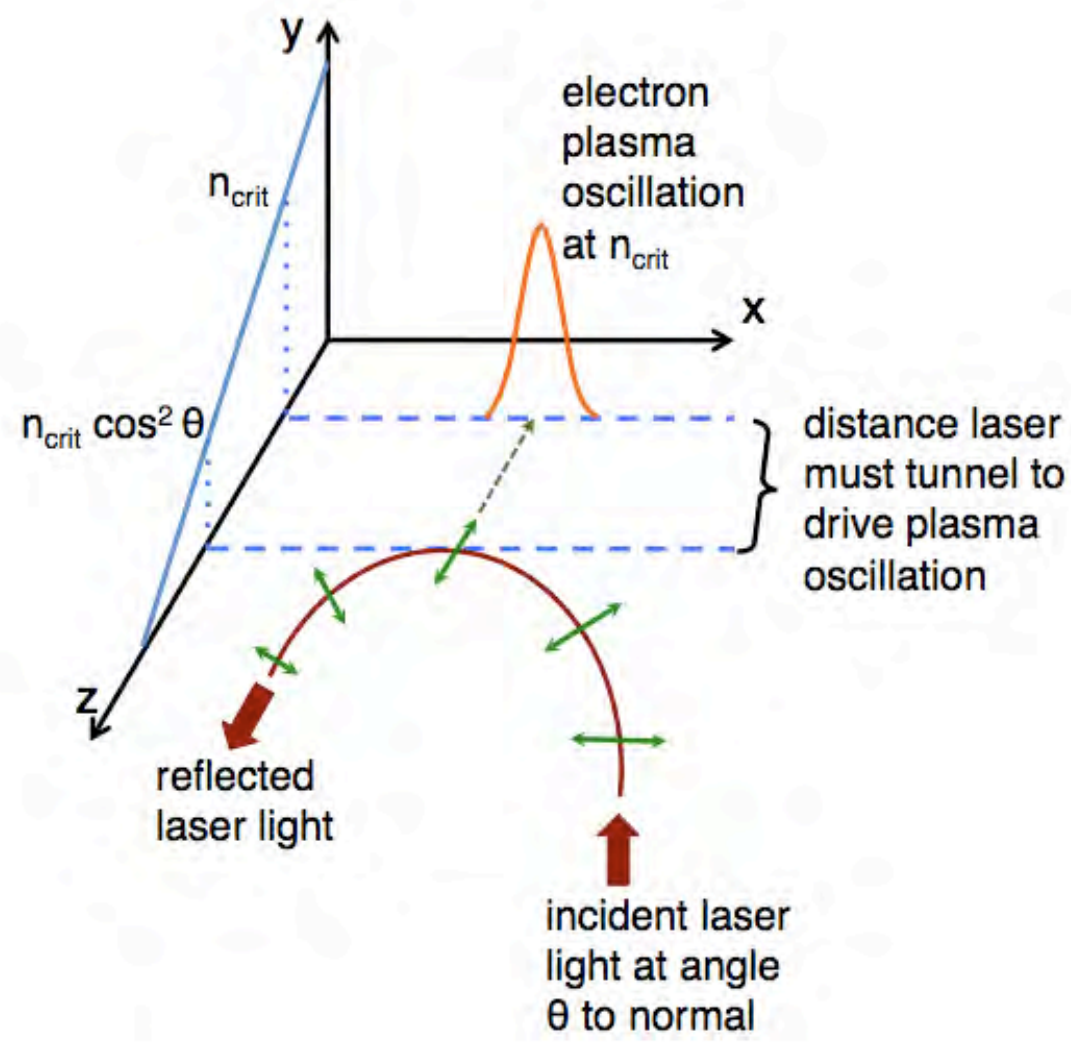

Figure 2.2: A schematic interpretation of resonance absorption. For $p$-polarized light, the electric vector of the light wave lies in the plane of incidence. The electric field can tunnel up the plasma density gradient to launch electron-plasma waves at the critical surface.

sorption of $\sim 50 \%$ could be obtained at an optimal angle of incidence given by

$$
\left(k_{0} L\right)^{2 / 3} \sin ^{2}\left(\theta_{o p t}\right) \simeq\left(\frac{1}{2}\right)^{2 / 3}
$$

exciting a population of very energetic electrons that would form a Maxwellian "tail" superimposed on the initial background temperature. Simulations[20, 21] showed that the temperature, $T_{h o t}$, of these hot electrons would scale as

$$
T_{h o t} \approx 10\left[T_{k e V} I_{15} \lambda_{\mu}^{2}\right]^{1 / 3} \mathrm{keV}
$$

where $T_{k e V}$ is the background electron temperature in $\mathrm{keV}, I_{15}$ is the laser intensity in units of $10^{15} \mathrm{~W} / \mathrm{cm}^{2}$, and $\lambda_{\mu}$ is the laser wavelength in microns. 
Because the energy is transferred from the laser electric field to the thermal energy of the plasma through the damping of the oscillations (by either collisional or collisionless processes), this absorption persists even when the classical collision frequency, $\nu_{e i}$, is small. Since $\nu_{e i} \propto T_{e}^{-3 / 2}$, resonance absorption will dominate over inverse bremsstrahlung at high laser intensities (high plasma temperatures), as well as at long laser wavelengths ( $n_{\text {crit }}$ occurs at a lower density).

\subsubsection{Vacuum Heating}

In the case of a very steep density profile, the scalelength of the plasma, $L$, can actually be less than the wavelength of the laser. This means that the average pathlength of the electrons is longer than $L$, and so the plasma will be unable to support the resonant oscillations of the electron-plasma waves near the critical surface. However, because the vacuum-plasma interface is so sharp, electrons near this boundary will be directly exposed to the laser field. An electron which is dragged out into the vacuum by the oscillating field will be reversed and accelerated back into the plasma when the field changes direction. Because the plasma is highly overdense (i.e., many times $n_{\text {crit }}$ ), the electric field only penetrates to a skin depth $\left(\sim c / \omega_{p}\right)$, so the electron can continue on into the target until it is eventually absorbed through collisions.

This process is called vacuum heating (also known as Brunel, or even "not-soresonant, resonance absorption" [22].) The laser light is nonresonantly coupled into electrostatic plasma waves, and plasma electrons effectively acquire kinetic energy directly from the laser field.

An analytical model developed by Brunel can be described in a simplified form as the "capacitor approximation":

If we assume an electric field is incident with some angle $\theta$ on an initially smooth, mirror-like front surface of the target, a component of the laser electric field, $E_{L}$, will be normal to the surface. Call this $E_{d}$, or the driving electric field, which will propel the electrons to oscillate across their equilibrium positions. Then,

$$
E_{d}=2 E_{L} \sin \theta
$$




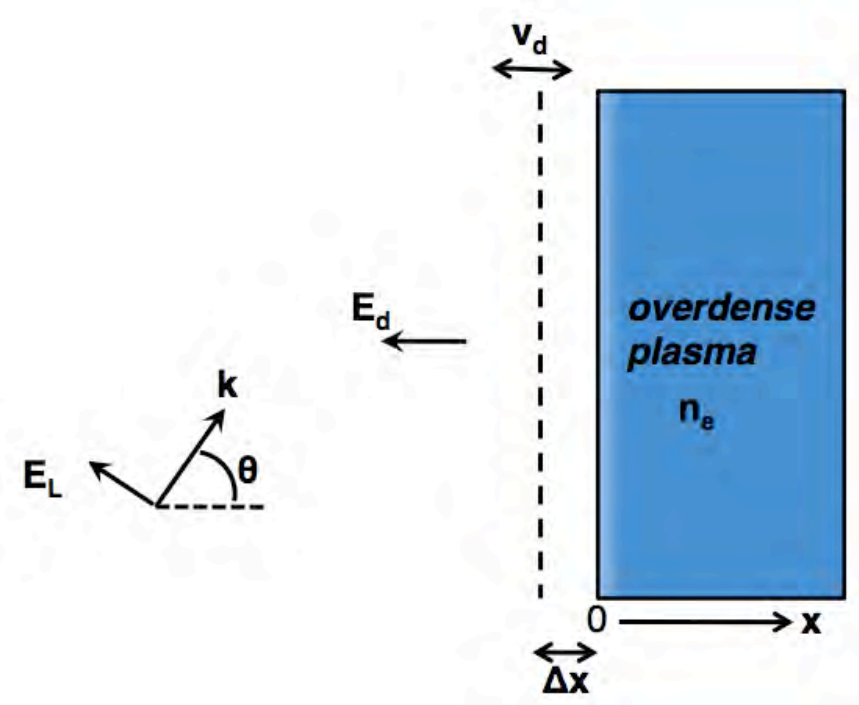

Figure 2.3: Simplified capacitor model of vacuum heating absorption.

If a sheet of electrons is pulled out a distance $\Delta x$ from its initial position, then the surface number density of the sheet is $\Sigma=n_{e} \Delta x$. An electric field will be created between $x=-\Delta x$ and $x=0$, as

$$
\Delta E=-4 \pi e \int_{-\Delta x}^{0} n d x=4 \pi e n_{e} \Delta x=4 \pi e \Sigma .
$$

Equating this to the driving electric field above, and solving for $\Sigma$, we get:

$$
\Sigma=\frac{E_{L} \sin \theta}{2 \pi e} .
$$

The charge sheet will continue to accelerate until it returns to its original position, by which it will have acquired a velocity, $v_{d} \simeq 2 v_{\text {osc }} \sin \theta$, where $v_{\text {osc }}$ is again the electron quiver velocity. If all the electrons in the sheet are then "absorbed" by the solid, where the energy density absorbed from laser light by the electrons is simply

$$
\epsilon_{a b s}=\frac{1}{2} \Sigma m_{0} v_{0}^{2}
$$

then the average energy density absorbed per laser cycle is

$$
\begin{array}{r}
P_{a b s}=\frac{\Sigma}{\tau} \frac{m v_{d}^{2}}{2} \\
\approx \omega_{0} \frac{E_{L} \sin \theta}{4 \pi e} m v_{d}^{2} .
\end{array}
$$


The incoming laser power was described as $P_{L}=c E_{L}^{2} \cos \theta / 8 \pi$, so the fractional absorption rate is:

$$
\eta_{v h} \equiv \frac{P_{a b s}}{P_{L}} \propto \frac{v_{o s c}}{c} \frac{\sin ^{3} \theta}{\cos \theta},
$$

Absorption scales linearly with $v_{o s c} / c$, so more absorption would be expected at higher $I \lambda^{2}$, and for larger angles of incidence. 1-D PIC simulations by Gibbon and Bell [23] showed that vacuum heating began dominating over resonance absorption for $L / \lambda<0.1$. Across a range of irradiances, and $L / \lambda$, absorption was found to peak at $75 \%$ for $L / \lambda=0.1$, and $I \lambda^{2}=10^{16}$ at a $\theta=45^{\circ}$. Absorbed energy was carried away by a hot electron population with $T_{h o t} \sim\left(\mathrm{I} \lambda^{2}\right)^{1 / 3-1 / 2}$

\subsection{3 j $\times$ B Heating}

With very intense laser light incident on a sharp plasma slab, the relativistic ponderomotive force can drive a significant oscillating electrostatic field. This $\mathbf{j} \times \mathbf{B}$ force[24] is due to the penetration of the laser $\mathbf{E}$ and $\mathbf{B}$ fields a skin depth into the overdense plasma. Physically, this can be interpreted as the laser light pushing on the plasma with a force that varies at $2 \omega_{0}$. This will accelerate the electrons into the plasma twice every laser cycle (i.e., at twice the laser frequency), giving the electrons a nonadiabatic kick into the plasma slab resulting in heating.

The ponderomotive force, derived above as Eq. (2.16), was written as:

$$
\mathbf{F}_{p}=-\frac{e^{2}}{4 m \omega_{0}^{2}} \nabla \mathbf{E}^{2}(\mathbf{x})
$$

For a linearly polarized light wave with an electric field amplitude $\mathbf{E}=E_{L}(x) \sin \left(\omega_{0} t\right)$, the ponderomotive force expression becomes

$$
\mathbf{F}_{p}=-\frac{m}{4} \frac{\partial}{\partial x} v_{o s c}^{2}(x)\left(1-\cos \left(2 \omega_{0} t\right)\right)
$$

where $v_{o s c}=\frac{e E_{L}}{m \omega_{0}}$. The first term is the static ponderomotive force, which produces steepening of the density profile, while the second term describes the oscillating electrostatic field that heats the electrons nonadiabatically.

Another way to interpret the $\mathbf{j} \times \mathbf{B}$ is to derive the potential for the electron fluid momentum: 
Again, the equation of motion for an electron fluid element in the plasma is

$$
\frac{\partial \mathbf{p}}{\partial t}+\mathbf{v} \cdot \nabla \mathbf{p}=-e\left[\mathbf{E}+\frac{\mathbf{v} \times \mathbf{B}}{c}\right]
$$

where $\mathbf{p}=\gamma m \mathbf{v}$ in the relativistic regime.

Then, rewriting our equation of motion in terms of the vector potential, $\mathbf{A}$ (where $\mathbf{B}=\nabla \times \mathbf{A}$ ) and the electrostatic potential, $\phi$ (where $\mathbf{E}=-\frac{1}{c} \frac{\partial \mathbf{A}}{\partial t}-\nabla \phi$ ), and substituting in the momentum vector for the velocity, we can rewrite Eq. $(2.29)$ as

$$
\frac{\partial \mathbf{p}}{\partial t}+\frac{\mathbf{p} \cdot \nabla \mathbf{p}}{\gamma m}=-e\left[\left(-\nabla \phi-\frac{1}{c} \frac{\partial \mathbf{A}}{\partial t}\right)+\frac{\mathbf{p} \times \nabla \times \mathbf{A}}{\gamma m c}\right] .
$$

Decomposing the momentum into transverse and longitudinal components, $\mathbf{p}=$ $\mathbf{p}_{t}+\mathbf{p}_{L}$, and taking $\mathbf{A}$ to only have a transverse component that varies in the longitudinal $(\hat{z})$ direction, we get

$$
\frac{\partial}{\partial t}\left(\mathbf{p}_{\mathbf{t}}-\frac{e}{c} \mathbf{A}\right)+\frac{\mathbf{p}_{\mathbf{L}}}{\gamma m} \frac{\partial}{\partial z}\left(\mathbf{p}_{\mathbf{t}}-\frac{e}{c} \mathbf{A}\right)=0
$$

for the transverse direction, giving the transverse momentum a form,

$$
\mathbf{p}_{\mathbf{t}}=\frac{e}{c} \mathbf{A}
$$

The longitudinal portion of the fluid equation then looks like,

$$
\frac{\partial p_{L}}{\partial t}+\frac{\mathbf{p} \cdot \nabla \mathbf{p}}{\gamma m}=e \nabla \phi-\frac{e}{\gamma m c} \frac{\mathbf{p} \times \nabla \mathbf{A}}{\gamma m c}
$$

which simplifies to

$$
\frac{\partial p_{L}}{\partial t}=e \nabla \phi-m_{0} c^{2} \nabla(\gamma-1)
$$

where (as defined above) $\gamma=\sqrt{\left(1+a_{0}^{2}\right)}$ for circularly polarized light and $\gamma=$ $\sqrt{1+a_{0}^{2} / 2}$ for linearly polarized light.

The first term on the right hand side is the standard electrostatic force, and the second term is the relativistic ponderomotive force, with a potential,

$$
U_{p}=(\gamma-1) m_{0} c^{2}
$$

The energy gained by the electrons due to this mechanism will scale accordingly, giving a "temperature" to the hot electrons as[25, 26]

$$
T_{\text {hot }} \approx\left(\sqrt{1+\frac{I \lambda_{\mu m}^{2}}{2.8 \times 10^{18}}}-1\right) 511 \mathrm{keV}
$$


for linear polarization. (The factor in the denominator becomes a $1.37 \times 10^{18}$ in the case of circular polarization.)

As the magnitude of the $\mathbf{j} \times \mathbf{B}$ force is proportional to the ratio of $n_{\text {crit }} / n_{e}$, the higher the electron density of the plasma slab, the less efficient the heating due to j $\times$ B. Malka and Miquel[26] demonstrated experimental verification of the $T_{\text {hot }}$ scaling for $\mathbf{j} \times \mathbf{B}$, finding a hot temperature of $1 \mathrm{MeV}$ for a $10^{19} \mathrm{~W} / \mathrm{cm}^{2}$ and an absorption efficiency from laser energy into hot electrons of $10-20 \%$.

\subsection{Generation and Transport of Relativistic Elec- tron Beams in Solid Targets}

As we have seen, impressive laser intensities can drive electrons very relativistically, creating a population of "hot" or "fast" electrons with kinetic energies significantly higher (hundreds of $\mathrm{keV}$ to several $\mathrm{MeV}$ ) than the mean (bulk) electron energy (tens or hundreds of eV).

For standard ICF schemes, the generation of fast electrons is viewed as a dangerous phenomenon, because these electrons can induce preheating in the target and therefore reduce the degree of achievable fuel compression. However, in fast ignition, the fast electrons are instead put to good use by delivering a large amount of energy within a time negligible with respect to hydrodynamical time scales.

The mechanisms discussed above for generating hot electrons depend strongly on parameters such as the temporal and spatial laser intensity profile, the preplasma scalelength, and target material and geometry. Once produced, the propagation of the hot electrons is governed not only by collisions with ions and other electrons, but also by self-generated electric and magnetic fields, as well as the return current that must arise. These parameters, as well as some of the experimental results in studying them, are discussed here. 


\subsubsection{Hot Electron Conversion Efficiency}

Naturally, the efficiency with which laser energy can be converted to hot electron energy is one of the most important parameters governing the feasibility of fast ignition. Over the years, numerous experiments have attempted to determine the absolute conversion efficiency. Results vary depending on the laser facility at which the experiment was carried out on, but in most cases, efficiencies tend to range around $20-50 \%$ for intensities $>10^{19} \mathrm{~W} / \mathrm{cm}^{2}[27,28,29]$, with the efficiency scaling roughly as $0.3-0.45$ the power of the laser intensity[30, 28]. The large range can likely be attributed to differing laser spot sizes, spot qualities, laser prepulse, or target characteristics, and the definition used by the authors for each of those parameters (i.e., how much energy is actually contained within a focal spot of a certain size, etc.)

The other uncertainty in conversion efficiency measurements is the method of interpretation used by the authors. In most hot electron conversion efficiency experiments, the number of hot electrons is inferred from the number of K $\alpha$ photons recorded, with the assumption that the cross-section for $\mathrm{K} \alpha$ production is nearly constant for a large range of electron energies. These $\mathrm{K} \alpha$ photons are generally emitted from buried fluor layers which are then used to estimate the number of electrons generated at the front surface of the target. A Monte Carlo (or similartype) model simulates the propagation of the electrons and approximates the initial number of electrons that could reach the fluor layer. However, at best, these Monte Carlo models are simplistic descriptions of the physics, where electron distributions and divergence angle must be assumed, and collective effects (such as fields) are ignored.

Using the Nova laser facility at LLNL $(1.06 \mu \mathrm{m}$ light, 12 - $30 \mathrm{~J}$ in $400 \mathrm{fs}, 15 \mu \mathrm{m}$ FWHM focal spot for an incident laser intensity $\sim 2-4 \times 10^{19} \mathrm{~W} / \mathrm{cm}^{2}$ ), Wharton et al.[27]'s experiment used layers of molybdenum buried at various depths under $\mathrm{CH}$, $\mathrm{Al}$, or $\mathrm{Cu}$. Using Monte Carlo modeling, Wharton et al. determined a coupling of $20-30 \%$ from laser energy into forward propagating electrons that varied as a function of laser intensity, but not on the target material.

Yasuike et al.[28] performed a similar experiment to measure coupling efficien- 


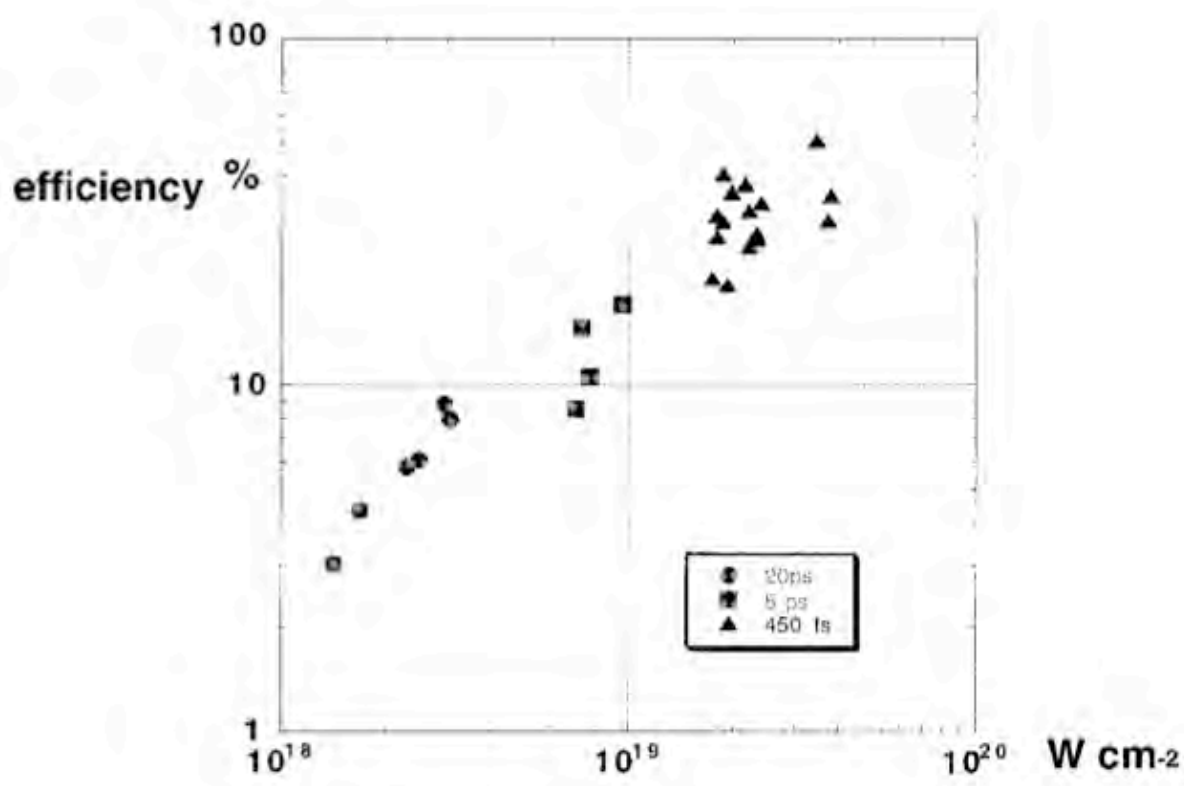

Figure 2.4: Conversion efficiency of laser energy into hot electron energy plotted against intensity on target. The 20 and 5 ps points are for laser energies of 200-400 $\mathrm{J}$, and 450 fs data are with 15-20 J energies. Figure taken from Key, Physics of Plasmas 1998[31].

cies as a function of laser intensity, but in this case held the laser energy constant ( $\sim 400 \mathrm{~J})$ but varied the laser pulselength $(0.5-20 \mathrm{ps})$ and found a coupling efficiency that maxed at $\sim 50 \%$ at $3 \times 10^{20} \mathrm{~W} / \mathrm{cm}^{2}$. At $1 \times 10^{19}$, coupling was $\sim 18 \%$, and $\sim 12 \%$ at $2 \times 10^{18} \mathrm{~W} / \mathrm{cm}^{2}$.

A combination of bremsstrahlung and $\mathrm{K} \alpha$ measurements on planar targets were made by Chen et al.[29] on the Titan laser in the range of $3 \times 10^{18}-8 \times 10^{19} \mathrm{~W} / \mathrm{cm}^{2}$. From Monte Carlo modeling of the target emission, conversion efficiencies into 1 $3 \mathrm{MeV}$ electrons of $3 \%$ - 12\%, representing $20 \%$ - $40 \%$ total conversion efficiencies were inferred.

Key et al.[31] also describe an experiment in which $\mathrm{Al}$ targets irradiated over a fairly large laser intensity range $\left(10^{18}-10^{20}\right)$ give conversion efficiencies of $5-15 \%$. These results support the argument that conversion efficiency appears to depend on laser intensity, and very weakly or none at all on laser pulselength when other parameters are held constant. 


\subsubsection{Hot Electron Temperature Scaling}

A number of different expressions from the literature describe the hot electron temperature as a scaling with laser intensity. The expression $T_{h o t} \propto\left(I \lambda^{2}\right)^{y}$ has been found, in general, to hold true across a number of laser experiments, with the exponent $y \approx 1 / 3$ for lower laser intensities and $y \approx 1 / 2$ for higher intensities.

Beg et al.[32] empirically demonstrated a scaling of

$$
T_{h o t} \approx 100 \mathrm{keV}\left(\frac{I \lambda^{2}}{10^{17}}\right)^{1 / 3}
$$

where $\lambda$ is the laser wavelength in microns and $I$ is the laser intensity in $\mathrm{W} / \mathrm{cm}^{2}$. This scaling applies for laser intensities up to $10^{19} \mathrm{~W} / \mathrm{cm}^{2}$, and can be attributed to resonance or vacuum heating.

At higher laser intensities $\left(>10^{19}\right), \mathbf{j} \times \mathbf{B}$ dominates to give a ponderomotive scaling, described by Wilks et al.[25] and Malka et al.[26] as in equation 2.36.

Recent theoretical work by Haines et al.[34] provides a small relativistic correction to the $\left(I \lambda^{2}\right)^{1 / 3}$ scaling for cases of when the precursor plasma density profile is swept up, and therefore steepened by the main laser pulse. The Ponderomotive, Beg, and Haines scalings are co-plotted in Fig. 2.5

However, it is an oversimplistic view to simply assign a $T_{\text {hot }}$ based on a single $I \lambda^{2}$, since in reality, a laser pulse is rarely a flat-top, but is more generally some type of gaussian with a peak energy, and wings of much lower energy. Further, the laser pulse will often be incident on a preformed plasma which will continuously evolve as it is interacting with the laser itself. In the case of a long density profile $\left(L>>c / w_{p e}\right)$, the skin depth is longer than the electron excursion length, and the electron can be "fully" accelerated by the laser field. If the plasma density gradient is small, however, this means the electrons do not have the distance to fully accelerate, leading to a reduction in their average energies. This small density gradient could either be due to a very small initial prepulse, or, as shown by Kemp et al.[35], at laser intensities around $10^{20} \mathrm{~W} / \mathrm{cm}^{2}$, the ponderomotive pressure of the beam could compress the plasma at $>n_{\text {crit }}$, steepening the interface. Because skin depth is inversely proportional to the density at the critical surface, the electron spectrum temperature drops by a factor of $\sqrt{\left(\gamma n_{\text {crit }}\right) / n_{p}}$, where $n_{p}$ is 


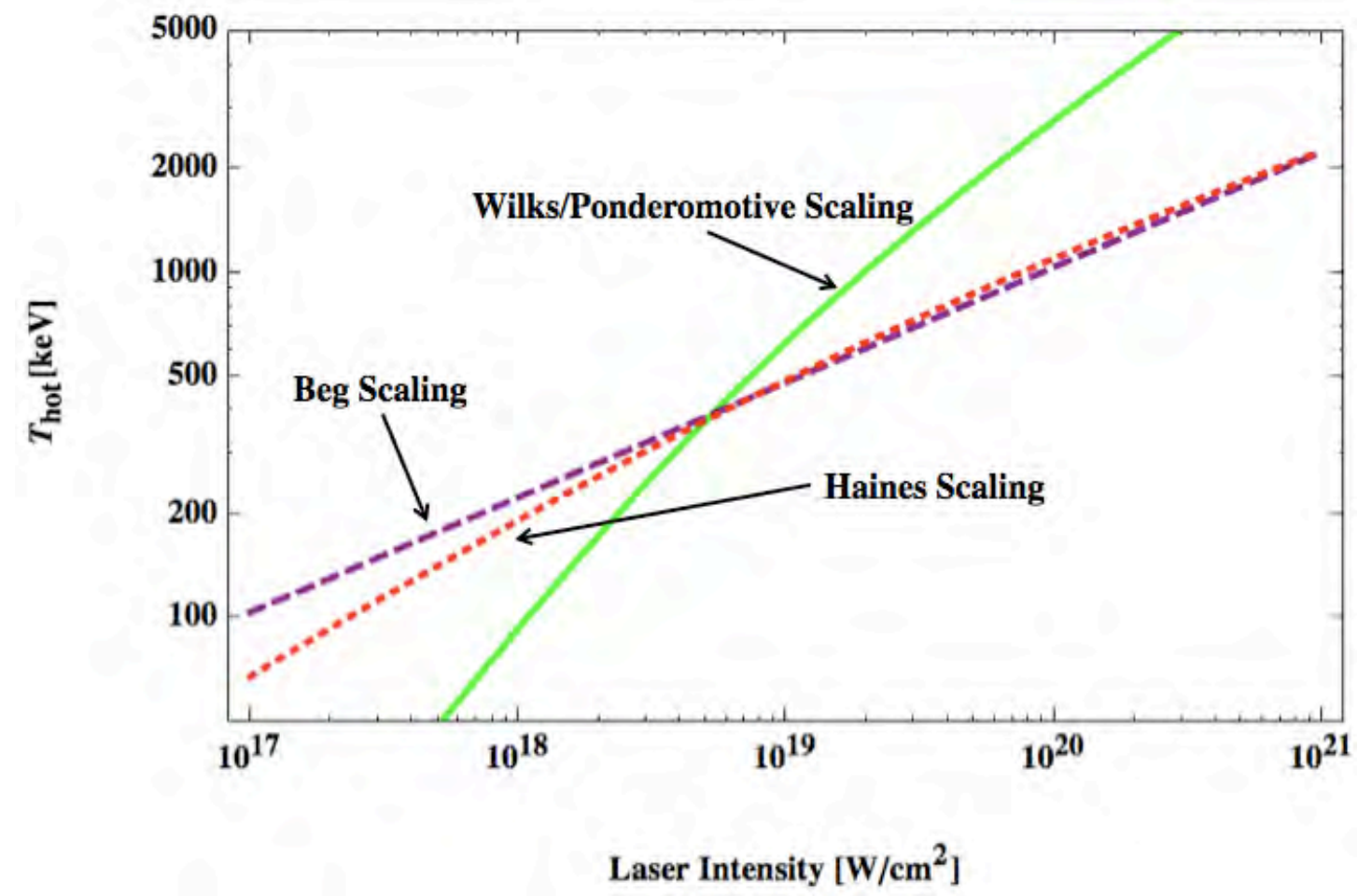

Figure 2.5: Predicted electron energy spectrum $T_{\text {hot }}$ based on Ponderomotive (solid, green), Beg (dashed, purple), and Haines (dotted, red) scaling. Experimental evidence seems to show that below laser intensities of $10^{19} \mathrm{~W} / \mathrm{cm}^{2}, T_{\text {hot }}$ scales with Beg or Haines[29, 32], and above that intensity, with Ponderomotive[33].

the electron density in the steepened plasma slab. This was further illustrated in PIC calculations by Chrisman et al. [36].

Another complication is that the definition of fast electron temperature used in the various literature is not always the same, and depends on the choice of the fast electron distribution. This issue was discussed by Key et al.[31], Davies [37], and Batani[38]. A purely exponential distribution,

$$
f(E) \approx \exp \left(-E / T_{h o t}\right)
$$

is often used, which is not a Maxwellian distribution, and what is called the $T_{\text {hot }}$ is actually the fast electron mean energy $\langle E\rangle$. A true Maxwellian has the form

$$
f(E) \approx E^{1 / 2} \exp \left(-E / T_{h o t}\right),
$$

which gives an mean electron energy of $\langle E\rangle=3 / 2 T_{\text {hot }}$. A 3D relativistic 
Maxwellian looks like

$$
f(E) \approx E^{2} \exp \left(-E / T_{h o t}\right)
$$

with $\langle E\rangle=3 T_{\text {hot }}$. Obviously, this shows that the type of electron distribution assumed in inferring data can change the number of electrons at low energies, and result in a wide range of possible mean electron energies for a given $T_{h o t}$. However, the distribution has been shown to have a relatively small effect on the absorption[37].

\subsubsection{Collisional versus Collective Effects in Propagation}

As the fast electrons propagate through the target material, the physics of the penetration is determined by a combination of both collisional (i.e., stopping and scattering of the electrons) and collective (i.e., electromagnetic) effects.

Collisional effects can be described in terms of stopping power, or the energy loss of the electrons per unit path length, $d E / d x$, due to collisions with bound and other free electrons[39]. Scattering can also occur through deflections with other particles[40], altering the trajectory of the electron and therefore its penetration. Collisional effects will dominate in cases where the total number of hot electrons is relatively small and the average hot electron energy is very large (such that the stopping distance is $>>$ larger than the penetration depth due to collective effects).

However, it is often the case in these ultra-intense laser-plasma interactions that a large number of hot electrons is generated, and the total electron current is quite large. The current of fast electrons flowing into the target will rapidly ionize the material and create a plasma with resistivity $\eta$. Then, in order for this current to propagate, charge neutrality becomes an issue, and must be satisfied as

$$
j_{\text {total }}=j_{\text {fast }}+j_{\text {return }} \cong 0 \text {. }
$$

$j_{\text {return }}$ will be directly affected by the resistivity, giving rise to a resistive electric field, $E=\eta j_{\text {return }}=-\eta j_{\text {fast }}$. This electric field can also be defined as the gradient of the Ohmic potential, whose direction is precisely that which transfers energy 
from the fast electrons to the background electrons. This will decelerate the fast electrons and convert a portion of their energy into Ohmic heating, $\eta j_{\text {return }}^{2}[41]$.

A "back-of-the-envelope," numeric explanation for why a $j_{\text {return }}$ must exist is provided in the paper by Bell et al.[42]. The paper starts with a 1 ps, $30 \mu \mathrm{m}$ focal spot laser pulse and an absorbed laser intensity of $10^{18} \mathrm{~W} / \mathrm{cm}^{2}$. This puts $\sim 7 \mathrm{~J}$ incident on an aluminum target, producing $10^{14}$ fast electrons with a temperature $T_{0}=200 \mathrm{keV}$. Assuming the electrons enter the target collimated to the focal spot, the current will be on the order of $24 \mathrm{MA}$, generating a cylindrical magnetic field of $3200 \mathrm{MG}$. The collisional stopping range for a $200 \mathrm{keV}$ electron in aluminum is $215 \mu \mathrm{m}$, giving a root-mean-square (RMS) range of $215 \mu \mathrm{m} / \sqrt{Z}=60 \mu \mathrm{m}$. Then if the current penetrated this RMS range into the target, the energy in the magnetic field would be $\sim 5 \mathrm{~kJ}$ !

This is a huge energy that is physically impossible, implying that a such a large current cannot be maintained, and therefore must be opposed by a $j_{\text {return }}$ that fulfills Eqn. 2.41. Because $j_{\text {return }}$ is composed of background thermal electrons, with much slower speeds than the hots, they must significantly outnumber the number of fast electrons. For this to be true at all spatial locations, either the fast electron current must be confined near the surface of the target by inductively or electrostatically generated electric fields, or return currents must be generated from the background plasma.

The penetration of the fast electrons is regulated by a diffusion coefficient that dictates how well the background plasma can provide a balancing return current. The continuity equation for fast electrons is

$$
\frac{\partial n}{\partial t}=\nabla \cdot\left(\frac{j_{\text {fast }}}{e}\right)=-\nabla \cdot\left(\frac{\sigma}{e} E\right)
$$

where we have substituted $j_{\text {fast }}=j_{\text {thermal }}=\sigma E$, with $\sigma$ being the conductivity of the plasma. Assuming the electron distribution is Maxwellian and confined by an electric field $E=-\nabla \phi$, the fast electron number density is $n \approx$ constant $\times$ $\exp \left(\phi / T_{h o t}\right)$, then

$$
E=-\frac{T_{h o t}}{n} \nabla n
$$


and substituting into Eqn. 2.42, we get

$$
\frac{\partial n}{\partial t}=\nabla \cdot\left(\frac{\sigma T_{h o t}}{e n} \nabla n\right)
$$

This gives us a diffusion constant of $D=\frac{\sigma T_{h o t}}{e n}$ showing that the coefficient is inversely proportional to the fast-electron density and that as the laser pulse progresses, the fast electrons that come later in time are inhibited from propagation by the build up of electron density at early times.

To quantify the inhibition of the fast electron penetration, Bell et al. further provides expressions for the hot electron density and penetration distance as a function of laser intensity and hot electron temperature. While these equations are highly simplified, and do not take into account the temporal and spatial evolution of the fast electron pulse, they do provide a useful gauge of the strength of the Ohmic stopping when compared to the collisional stopping range.

The hot electron density follows a form:

$$
n_{0}=\frac{2 I_{\text {abs }}^{2} \tau_{\text {laser }}}{9 e T_{\text {hot }}^{3} \sigma}
$$

and the Ohmic stopping scalelength, $z_{0}$ is defined to be

$$
z_{0}=\frac{3 T_{h o t}^{2} \sigma}{I_{a b s}},
$$

where $\tau_{\text {laser }}$ is in seconds, $T_{h o t}$ is in $\mathrm{eV}$ and $I_{a b s}$ is the absorbed laser intensity in units of $\mathrm{W} / \mathrm{m}^{2}$ to give $z_{0}$ in meters. The Ohmic stopping scalelength is the distance over which the potential change is equal to the mean energy of the fast electrons. $n_{0}$ is proportional to the laser intensity squared, while $z_{0}$ is inversely proportional to the laser intensity. This tells us that as laser intensity increases, so does conversion efficiency, but penetration depth goes down. In other words, if the flux of hot electrons goes up, so does the potential that arises to stop them.

Collisional stopping power increases with $Z$ and $\rho$, while electric inhibition decreases when $\sigma$ increases.

\subsection{Conclusions}

This chapter has introduced a few of the basic principles of how laser energy is absorbed and carried away by hot electrons in a plasma. Relativistic electrons 
can be generated and accelerated via a number of physical processes including resonance absorption, vacuum heating, and/or ponderomotive $\mathbf{j} \times \mathbf{B}$ acceleration. In the high intensity regime $\left(\mathrm{I} \lambda^{2} \geq 10^{15} \mathrm{~W} \mu \mathrm{m}^{2} \mathrm{~cm}^{-2}\right)$, the hot electron temperature generally follows a scaling of $T_{h o t} \propto\left(I \lambda^{2}\right)^{y}$, where $y$ typically varies between $\frac{1}{2}-\frac{1}{3}$. The number of fast electrons generated will then have an influence on the space charge that is set up, and as they propagate they will draw return current through resistive electric fields.

This thesis will focus on a number of these processes, namely the effect of large underdense plasmas on the absorption of the laser pulse and subsequent hot electron characteristics. Both experimental and modeling work was undertaken to try to understand some of the complex physics involved. 


\section{Chapter 3}

\section{The Titan and Vulcan Laser Facilities and Experimental Configurations}

\subsection{Introduction}

The advent of the ultra-high intensity, short-pulse laser has opened up new fields of research in the regimes of high energy and high density physics. In the interaction of such a laser with a solid target, the laser energy is absorbed within the laser skin depth and creates a rapidly evolving plasma with a lifetime comparable to the pulse duration of the laser and whose spatial scale is on the order of tens of microns. The plasma varies from zero to thousands of $\mathrm{eV}$ temperature, and approaches solid density.

The sharp spatial and temporal gradients of the rapidly evolving plasma pose unique challenges for diagnosis. In order to begin to understand the wealth of physics within a single laser shot on a solid target, a large array of diagnostic instruments are necessary to carefully diagnose both the laser and the interaction.

The experiments comprising this thesis were completed using the Titan laser at the Jupiter Laser Facility at the Lawrence Livermore National Laboratory (LLNL) and the Vulcan Petawatt laser at the Central Laser Facility (CLF) at the Ruther- 
ford Appleton Laboratory (RAL) in the U.K. Both are state-of-the-art, petawattclass lasers.

This chapter is organized as follows: first, a brief overview of some of the advanced technology that makes short pulse, ultra-high intensity lasers possible, followed by descriptions of the Titan and Vulcan laser systems. Then, laser diagnostics and target diagnostics used in the research of laser-plasma interactions, and consequently electron transport, are presented.

\subsection{The Technology behind Ultra-Short Pulse, Ultra-High Intensity Lasers}

The high laser intensities necessary for the types of experiments described in this thesis can be attributed to several key advancements in laser technology, namely, mode-locking[43], Optical Parametric Amplification (OPA)[44], and Chirped Pulse Amplification (CPA)[45].

Mode-locking[43], developed in the early 1970s, allows for the generation of extremely short pulses of light, on the order of pico- or femtoseconds. The length of a single pulse of laser light is set by two factors: the gain bandwidth (the range of frequencies the laser can operate over, dependent on the gain medium of the laser), and the resonant cavity of the laser. Within the resonant cavity, the light waves will bounce around, constructively and destructively interfering with themselves to form standing waves. These standing waves, or modes of the cavity, are the only frequencies allowed to oscillate within the cavity, while all others are suppressed by destructive interference. Each mode will oscillate independently, occasionally interfering, leading to random fluctuations in intensity. However, if the laser is mode-locked, all the modes will periodically interfere constructively, producing an intense burst of light within a time duration equivalent to the time it takes for the light to make one round trip of the laser cavity. Mode-locking can be achieved by using an electrical modulator in the cavity that produces a sinusoidal phase modulation of the light in the cavity, or by using a saturable absorber that

preferentially absorbs low intensity light while transmitting light of sufficiently 
high intensities.

Optical Parametric Amplification (OPA)[46, 44] uses a high gain non-linear crystal in the pre-amplification stage. The initially weak incident signal beam is amplified by an intense, fixed frequency pump beam that is simultaneously incident on the nonlinear crystal. Photons from the energetic pump beam are converted into lower energy signal photons, and an equal number of idler photons (photons with conjugate polarization). Since the pump energy is completely converted into either signal or idler photons, the crystal is not heated in the process. The amplified signal beam will then preserve the original spectral phase and spatial wavefront characteristics of the seed pulse. In order for OPA to be efficient, however, very high intensities on the order of tens of $\mathrm{GW} / \mathrm{cm}^{2}$ are required[47]. Therefore, the front end of femtosecond laser systems with nano or microjoules of energy are well suited for this process. A single pass through one or several pumped non-linear crystal replaces a multiple pass or regenerative amplification stage. This type of amplifier is more compact, can yield significantly higher gains than glass amplifiers, and produce a smoother beam profile.

The concept of Chirped Pulse Amplification (CPA)[45] permits the amplification of short pulses to petawatt power with no need to increase the spatial diameter of the beam. By expanding the beam in both time and space, the overall intensity can be reduced, and damage to optical components can be avoided (see Fig. 3.1). A seed laser pulse (generally of $<1 \mathrm{ps}$ in duration and $>1 \mathrm{~nm}$ in bandwidth, with a few nJ of energy) is stretched in time by a factor of 1000 to $10000 \times$ through a diffraction grating. This grating spectrally disperses the beam so that the low-frequency (red) component of the pulse travels a shorter path than the high-frequency (blue) component. The pulse is now positively chirped, in that the high-frequency component lags behind the low-frequency component. The spectrum of the pulse is unchanged, while the duration of the pulse increases by several orders of magnitude, and the intensity therefore decreases by that same factor. This stretched laser pulse can now be amplified, which in the case of many current high intensity lasers is done with a combination of regenerative preamplifiers and multipass power amplifiers. The regenerative amplifier provides much of 
the gain of the system $\left(\sim 10^{7}\right)$, which can bring the laser energy up to the mJ level, and then the power amplifiers amplify the pulse further to the $1 \mathrm{~J}$ level. This long, amplified pulse is then finally recompressed back to close to its original duration through a negative dispersion delay set of parallel diffraction gratings. Due to nonlinear dispersion effects and gain-narrowing, the final pulse will be somewhat lengthened from its original duration. The peak power increase achieved with CPA is directly related to the ratio to which the pulse was stretched and recompressed.

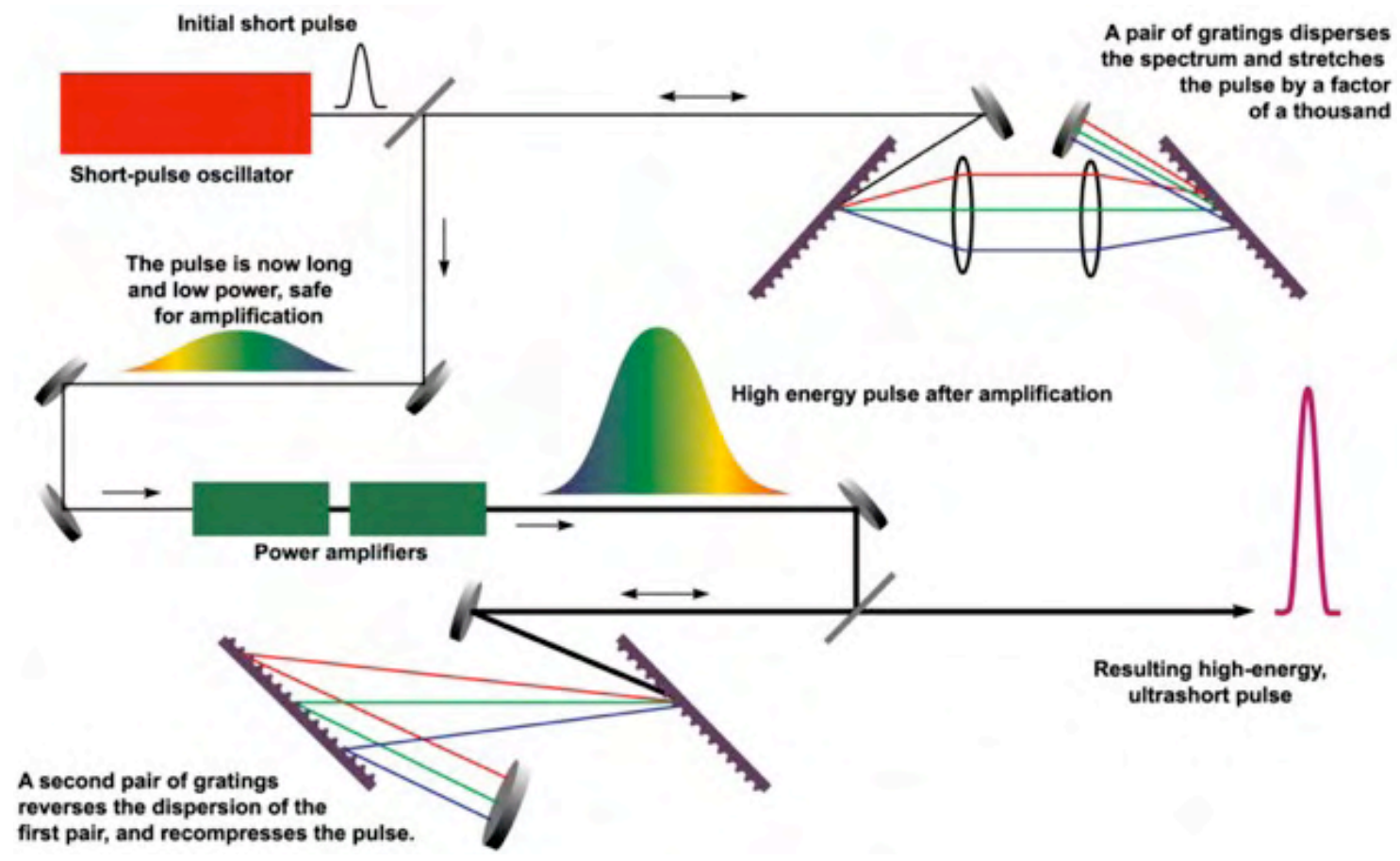

Figure 3.1: A typical chirped-pulse amplification (CPA) configuration. Image courtesy of LLNL S\&TR of September 1995.

When OPA is used in conjunction with CPA, it is called OPCPA, and can deliver high energy and intensity pulses with high contrast[48, 49]. A nanosecond OPCPA making a single pass through just several centimeters of gain material can obtain high gain with broad-bandwidth[50], resulting in 10-20 $\times$ shorter pulses than just CPA alone. Further, because the gain material is so short, the $B$ integral (distortion of the wavefront due to nonlinear phase) is reduced. 


\subsection{Formation of Laser Prepulse}

One of the drawbacks of the CPA technique is the presence of a temporal pedestal primarily due to amplified spontaneous emission (ASE) on the nanosecond timescale and main short pulse leakage on a subpicosecond timescale due to imperfect matching of optical elements in the laser chain.

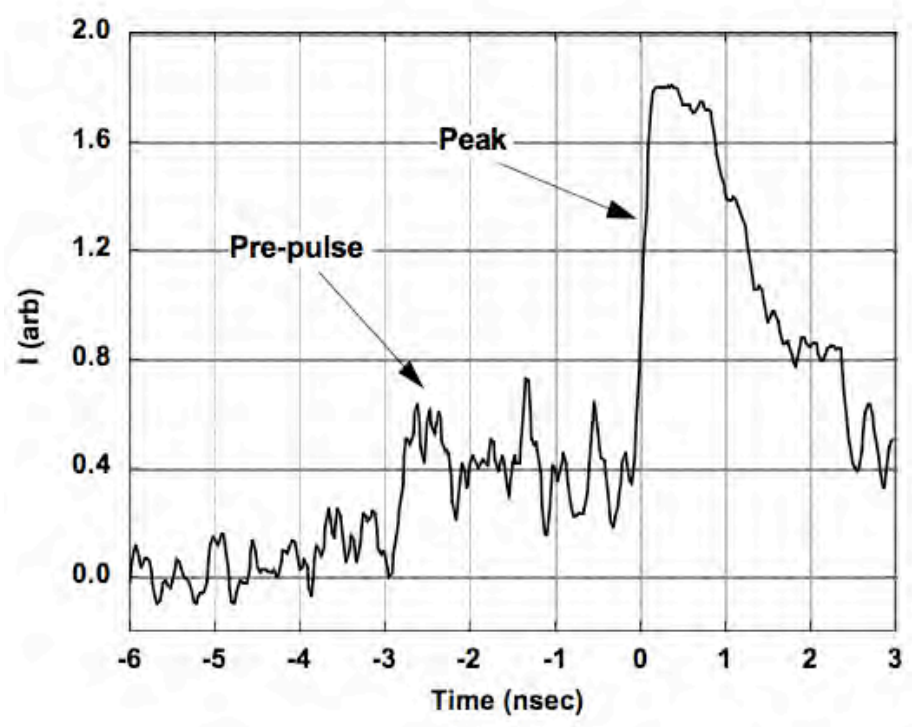

Figure 3.2: A typical intensity trace of a full system Titan short pulse laser shot. The ASE pedestal beings approximately $3 \mathrm{~ns}$ before the main pulse arrives at $\mathrm{T}=0$ ns. The intensity of the main pulse saturates the diode (evidenced by the flat top on the peak), and the gradual drop in peak pulse intensity is due to the finite rise and decay time of the diode. Image courtesy of P. Patel.

ASE, also known as superluminescence, occurs when the stored energy in the laser rod is greater than the saturated energy density of the rod material. All stages of the laser amplification contribute to ASE, but it is primarily dominated by pulses leaking from previous round trips in the regenerative amplifier. ASE is exactly as its name implies: it is spontaneous light, which means that it is isotropic and incoherent because it is seeded by many spontaneous photons that are randomly emitted in every direction. Those photons propagating in a direction close to the major dimension of the gain medium will be preferentially amplified by the various amplifying stages, and thus will compete for gain with the useful 
signal. Fast Pockels cells[51] and polarizers can be used as optical gates to remove ASE up to the leading edge of the pulse, but there will be some component of ASE that travels with the laser pulse that cannot be removed, which will manifest itself as a several nanosecond pedestal (equal to the switching time of a standard Pockels cell) in front of the short pulse.

The leakage of the short pulse, on the other hand, will generally have a pulse duration similar to the main pulse $(<\mathrm{ps})$, but will contain $\sim 30-40 \%$ of that of the ns pedestal.

As the peak intensity of these ultra-intense lasers routinely exceeds $10^{18}$, even in a system with fairly good intensity contrast of $10^{5}-10^{7}$, the pedestal is sufficient to create a preplasma that expands in front of the original surface of the target.

The presence of such a preformed plasma means that the main laser pulse interacts with an expanding and evolving plasma, rather than a clean solid target. Absorption of the laser is thereby affected, and this can modify the hot electron generation and its energy spectrum and divergence.

\subsection{The Titan PW OPCPA Laser}

The Titan laser at LLNL[52, 53] is a $1053 \mathrm{~nm} \mathrm{Nd:glass} \mathrm{laser} \mathrm{which} \mathrm{makes} \mathrm{use} \mathrm{of}$ both OPA and CPA to deliver laser pulses of up to $150 \mathrm{~J}$ in $500 \mathrm{fs}$ with an on-target intensity of $10^{20} \mathrm{Wcm}^{-2}$. A commercial Time Bandwidth Products, Inc. GLX-200 master oscillator first generates the seed pulse of $200 \mathrm{fs}$ and $1053 \mathrm{~nm}$. An Offner style pulse-stretcher then increases the pulse duration to $1.6 \mathrm{~ns}$ by spreading the different spectral components of the laser pulse (by giving one end of the spectrum a longer optical path than the other) using a $390 \mathrm{ps} / \mathrm{nm}$ chirp-parameter.

A two stage Optical Parametric Chirped Pulse Amplifier (OPCPA) serves as a front-end preamplifier which boosts the pulse energy from $1 \mathrm{~nJ}$ to $40 \mathrm{~mJ}$. The pump pulse for the OPCPA begins as a modulation of a CW oscillator, followed by regenerative amplification in a $3 \mathrm{~mm} \mathrm{Nd:YLF} \mathrm{rod,} \mathrm{and} \mathrm{a} \mathrm{4-pass} \mathrm{amplifier} \mathrm{with} \mathrm{two}$ $12.7 \mathrm{~mm} \mathrm{Nd:YLF}$ rods up to $1.5 \mathrm{~J}$ of $1.05 \mu \mathrm{m}$ output at $5 \mathrm{~Hz}$. A BBO $(\beta$-barium borate, or $\beta-\mathrm{BaB}_{2} \mathrm{O}_{4}$ ) crystal is the gain medium which produces $0.8 \mathrm{~J}$ of $0.53 \mu \mathrm{m}$ 


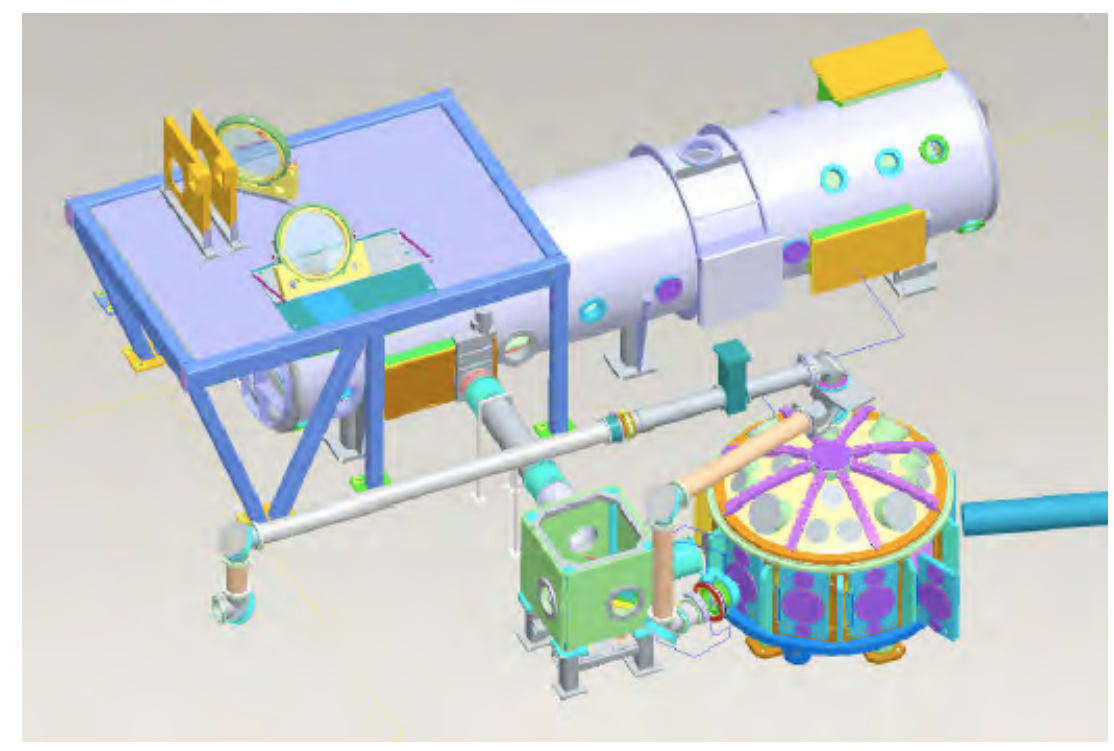

Figure 3.3: A 3-D model of the Titan laser at LLNL. Image courtesy of R. Van Maren.

light to pump the OPAs.

The $40 \mathrm{~mJ}$ OPCPA output is injected into flashlamp-pumped Nd:glass amplifiers including a $25 \mathrm{~mm}$ rod, two $50 \mathrm{~mm}$ rods, a set of double-pass $9.4 \mathrm{~cm}$ disks, a set of single pass $9.4 \mathrm{~cm}$ disks, and a $15 \mathrm{~cm}$ disk amplifer. Faraday rotators and pockels cells are used to prevent back reflections from propagating up the amplification chain and damaging the optics. A magnifying telescope then increases the beam size to $25 \mathrm{~cm}$. At this point, an adaptive optic system checks and adjusts the wavefront to match a preset optimal wavefront before transporting it to the vacuum compressor diffraction grating system. The deformable mirror has a set of actuators in a two-dimensional array to correct wavefront errors due to static aberrations of optics in the beamline and distortions caused by thermal gradients set up in the amplifiers during laser shots.

The compressor is a two-pass, two-grating system. The diffraction gratings are 1780 lines/mm, 40 x 80 cm Multi-Layer Dielectric (MLD) gratings with 95\% efficiency and which provide up to a $5 \times$ higher damage threshold than gold gratings. These gratings are operated at a $75^{\circ}$ angle of incidence, and give the beam its S-polarization. 
After compression, the beam is directed into the $2.5 \mathrm{~m}$ target chamber using two $45^{\circ}$ turning mirrors. There, an $\mathrm{f} / 3$ off-axis parabola focuses it down at target chamber center (TCC). At this point, the Titan short pulse laser has a nominal power of $300 \mathrm{TW}$, in a 500 fs full width at half maximum (FWHM) pulselength for a peak on target intensity of approximately $2 \times 10^{20} \mathrm{Wcm}^{-2}$.

The target chamber design is such that a high-energy (up to $1 \mathrm{~kJ}$ ) long-pulse (0.2 to $20 \mathrm{~ns}$ ) $2 \omega$ beam can also be focused into the chamber. The angle between the long pulse and short pulse beams can be varied in discreet positions between $35^{\circ}$ and $180^{\circ}$, or the long pulse can be injected collinear to the short pulse beam. The timing of the long pulse can be varied to come before, after, or overlapped with the short pulse beam.

\subsection{The Vulcan PW OPCPA Laser}

The Vulcan Petawatt Laser[54, 55] is a Nd:glass laser system capable of delivering up to $350 \mathrm{~J}$ on target in a pulse duration of $0.5 \mathrm{ps}$. Typically, $30 \%$ of the laser energy is contained within a $7 \mu \mathrm{m}$ diameter focal spot, giving a focused intensity in the mid $10^{20} \mathrm{Wcm}^{-2}$. The p-polarized laser has a wavelength of $1054 \mathrm{~nm}$.

A seed pulse of $1053 \mathrm{~nm}$ and $150 \mathrm{fs}$ duration is initiated by a commercial Kerr lens mode-locked Ti:sapphire oscillator. The pulses enter an Offner stretcher where they are lengthened to $4.8 \mathrm{~ns}$ before proceeding to to the OPCPA for preamplification to approximately the $10 \mathrm{~mJ}$ level over a wide bandwidth $(\sim 1 \mathrm{~mJ} / \mathrm{nm})$. The three-stages of the OPA are pumped by a 200-300 mJ, $4.5 \mathrm{~ns}$, frequency doubled pump pulse (generated by a $2 \mathrm{~J}, 1053 \mathrm{~nm}, 10 \mathrm{~Hz}$ YAG laser). The nonlinear gain medium used for the OPA stages is BBO crystal.

The pulse is then injected into the Vulcan rod and disk amplifier chain which uses Nd:phosphate and Nd:silicate media. First, a combination of 16, 25, and 45 mm rods amplify the pulses, while vacuum spatial filters clean the beam. The pulse is then amplified further using a double pass through a $108 \mathrm{~mm}$ diameter disk amplifier, and then single passes through a $150 \mathrm{~mm}$, then $3250 \mathrm{~mm}$ phosphate disks. An adaptive optic system is utilized to improve wavefront quality, employing 


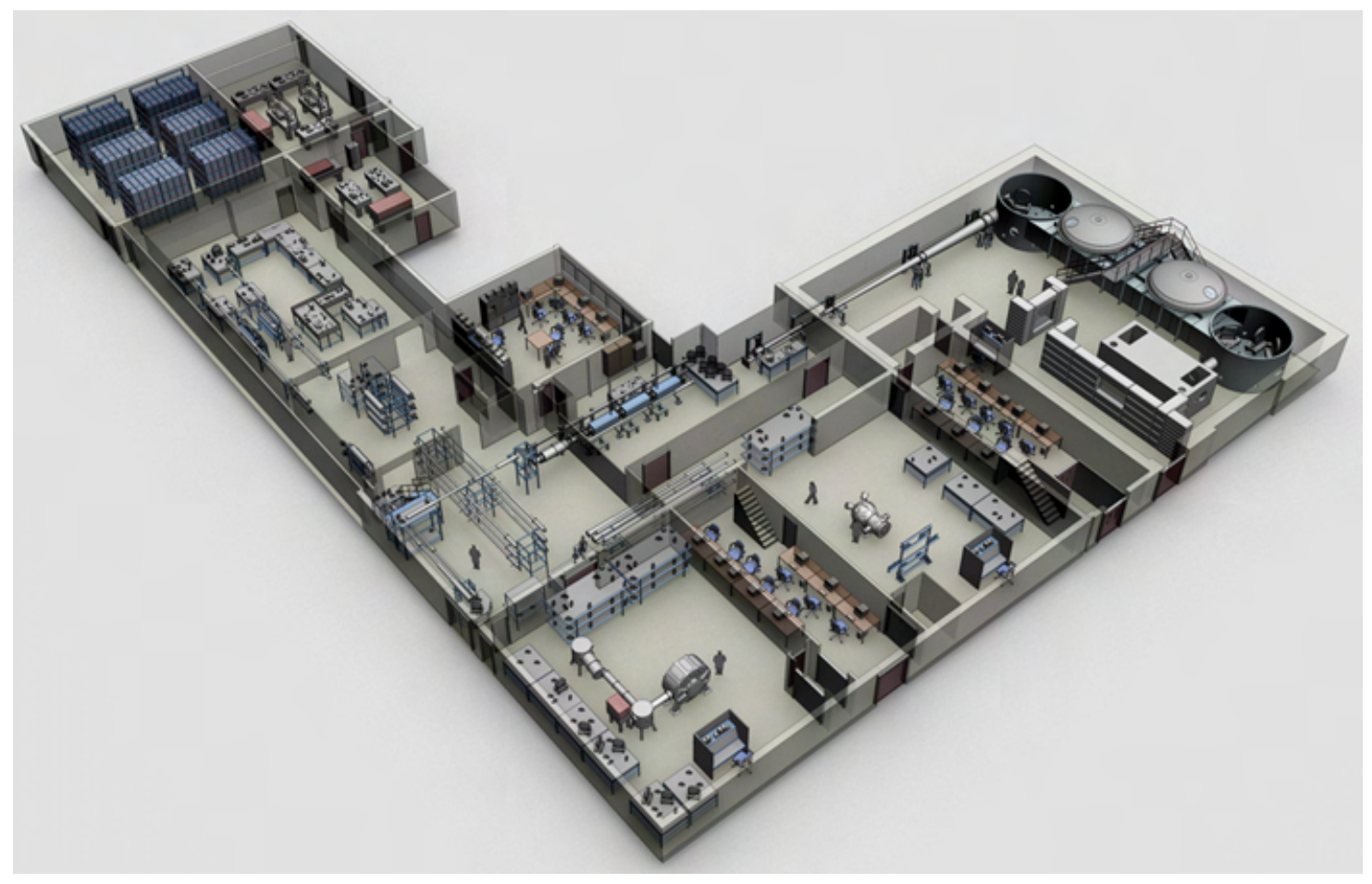

Figure 3.4: A 3D model of the Vulcan Laser Facility at the Rutherford Appleton Laboratory. Image courtesy of the Central Laser Facility.

a deformable mirror after the rod amplifiers, and a wave-front sensor behind the final disk amplifier before the petawatt spatial filter.

Finally, the pulse is recompressed by a factor of $>1000$ with a 1480 lines $/ \mathrm{mm}$ gold-coated holographic grating. The beam is $60 \mathrm{~cm}$ upon entering the chamber, and is incident on an $\mathrm{f} / 3.1$, off-axis parabola of $620 \mathrm{~mm}$ diameter. The $1.8 \mathrm{~m}$ focal length parabola then focuses the beam down at the center of the $4 \mathrm{~m} \times 2 \mathrm{~m} \times 2$ $\mathrm{m}$ rectangular target chamber.

\subsection{Experimental Diagnostics}

This research focuses on the necessity to understand the transport and energy deposition characteristics of fast electrons within solid material. However, the physics behind these phenomena is closely related to the characteristics of the laser, and so it is crucial to not only optimize each of those parameters, but to quantify 
and qualify them on each shot. Beyond the laser itself, the rapid interaction physics of the laser requires a unique diagnostic capability designed to extract the maximum amount of information from every shot. The following are some of the laser and target-interaction diagnostics used to address these needs.

\subsubsection{Characterizing the Laser}

\subsubsection{Laser Energy}

Laser energy is monitored on-shot using a calibrated calorimeter which measures the leakage ( $<$ a few percent) of the full-energy beam through a turning mirror immediately following the second compressor grating.

\subsubsection{Laser Focal Spot}

(a)

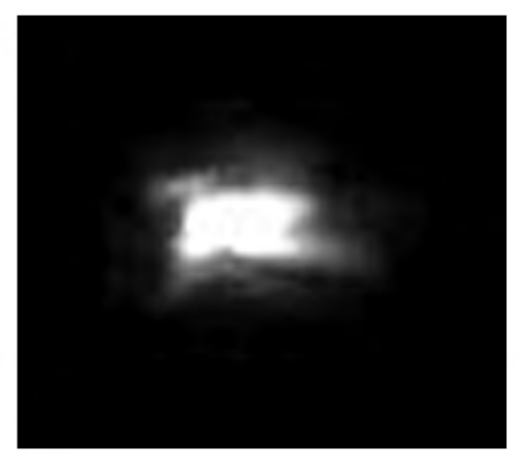

(b) 1

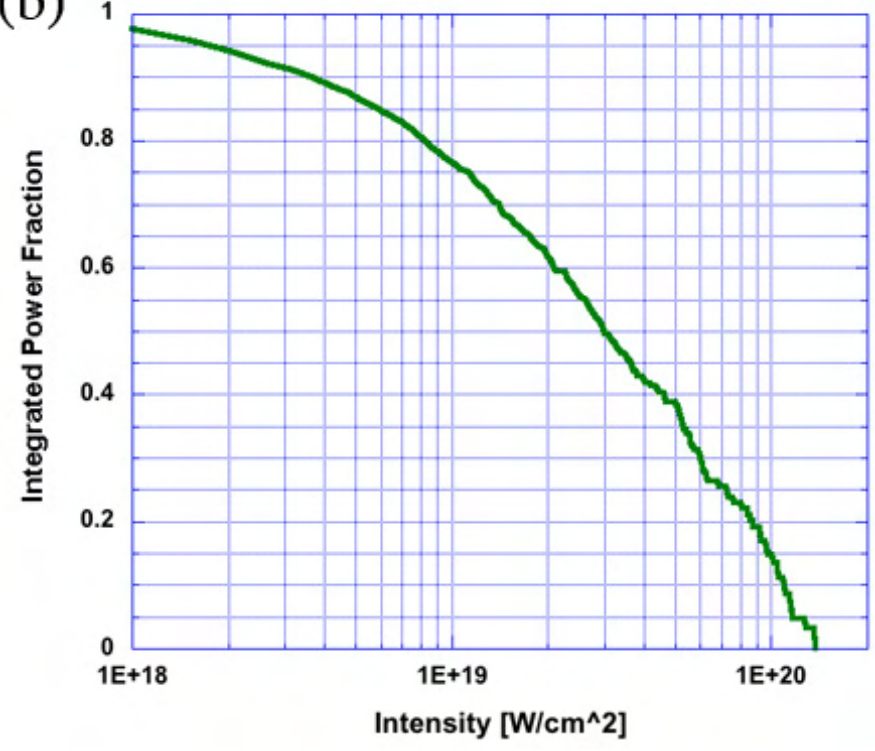

Figure 3.5: (a) The Titan OPCPA focal spot at TCC as imaged by the Equivalent Plane Monitor. (b) The integrated power fraction of the focal spot in (a).

On Titan, the on-shot laser focal spot intensity distribution is inferred through the use of an equivalent plane monitor (EPM). The EPM characterizes the effects of non-linear aberrations in the propagation optics and distortions induced by the 
pumping. The EPM takes the leakage $\left(\sim 10^{-5}\right)$ of the main laser through the final turning mirror before it reaches the focusing parabola and propagates the leakage beam through a series of optics designed to emulate the ones experienced by the full energy beam. A $30 \mathrm{~cm}$ diameter, $630 \mathrm{~cm} \mathrm{f} / 25$ focal length lens images the leakage onto a 16-bit CCD camera.

For the majority of shots, the full energy focal spot matched closely with direct measurements of the vacuum focal spot at TCC made at low power (using the unamplified, pulsed OPCPA beam).

\subsubsection{Laser Pulselength}

The pulse duration of the laser is generally referred to as the full width at half-maximum (FWHM) of the optical power versus time. The true minimum pulselength of the laser is governed not only by a combination of the gain media and resonant cavity size as referred to above, but also by how well the stretch and compression gratings are aligned.
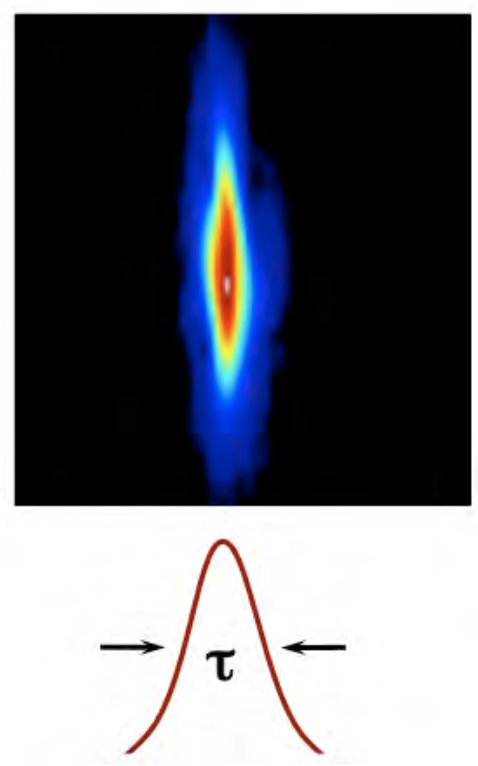

Figure 3.6: The intensity of the overlap of the split pulse gives the pulse width of the shot.

The pulse duration is monitored on a shot-to-shot basis with a second-order 
autocorrelator[56, 57]. First, a beam splitter splits the incoming laser pulse into two separate pulses. One of the pulses goes through a variable line delay, or stretcher grating, which can be moved to change the propagation distance, while the second pulse travels some static length. The two pulses are then recombined in a second-harmonic-generation crystal. The power of the mixed output is varied by adjusting the position of the stretcher grating, with maximum power indicating optimum compression. With a time delay that is too small or too large, the overlap of the two beams in the crystal will be reduced, and intensity will be weaker. By correlating the power with the path length difference, the pulse duration of the laser can be backed out.

\subsubsection{Laser Prepulse}

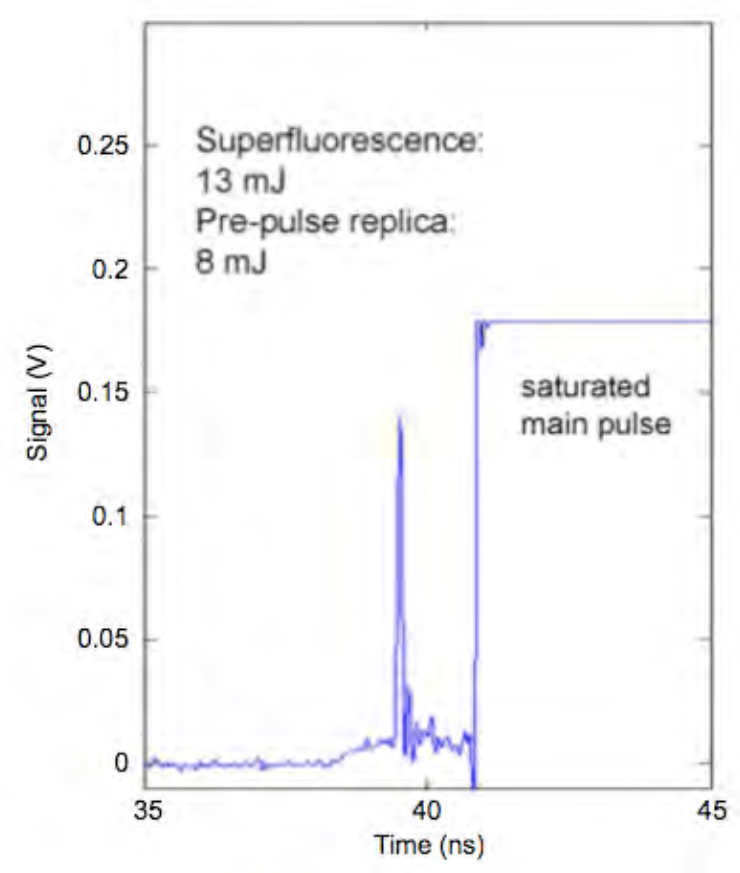

Figure 3.7: Oscilloscope trace showing the prepulse profile of a high-power laser pulse. The main pulse is saturated. Figure taken from MacPhee, Review of Scientific Instruments 2008[58].

To measure the laser prepulse, the leakage of the full aperture beam through 
a turning mirror directly following the compression grating is focused on a water cell[59]. This water cell acts as a filter for the high intensity light. The absorption of water becomes non-linear above a certain threshold $\left(\sim 10^{13} \mathrm{Wcm}^{-2}\right)$, thus breaking down if the prepulse intensity is too high and therefore protecting the fast diode. The diode is calibrated using the main pulse beam with appropriately calibrated neutral density filters. Laser prepulse is measured using a water-cell protected fast diode.

\subsubsection{Target Self-Emission Diagnostics}

\subsubsection{XUV Imager}

The Extreme Ultraviolet, or XUV, imager was developed to image the fast electron Planckian heating profiles of the rear surface of irradiated targets $[60,61]$. As the fast electrons pass through the target, they collisionally and Ohmically heat the target. The heated material quickly cools but provides a short burst of blackbody radiation. Typical target temperatures range from 10-300 eV, emitting blackbody radiation peaking in the soft x-ray to extreme ultraviolet range. This emission, which decreases exponentially as the plasma rapidly cools, is thus shortlived. Because radiation intensity varies so rapidly with temperature and the very short absorption length in the XUV, the time-integrated imaging of this radiation offers an excellent tool to measure the maximum surface temperature of dense plasma targets.

The XUV imaging diagnostic collects light within a solid angle determined by an aperture and relays this to a charge-coupled device (CCD) via two multilayer XUV mirrors that dictate the energy bandwidth. Two channels of the XUV diagnostic were employed: one optimized for $68 \mathrm{eV}$ light, and the other for $256 \mathrm{eV}$ light. By using two different channels imaging at slightly different energies, insights could be gained about the heating, expansion, and cooling characteristics of the target (because these energies are collected from different depths within the

plasma), as well as allow for the verification of thermal temperatures derived from each channel. The XUV imager will be discussed in more detail in Chapter 4. 


\subsubsection{XUV Flat Field Spectrometer}

An XUV flat-field spectrometer (FFS) [62, 63] was employed in conjunction with the XUV imagers to provide temperature measurements of the plasma plume that had expanded outwards from the target surfaces. The FFS records spectra in the 50-400 eV (30-250 $\stackrel{\circ}{\text { ) }}$ ) range by using a 1200 lines/mm concave diffraction grating set to a glancing angle of $4.6^{\circ}$. The use of a such a concave grating with variable line spacing allows for imaging of the spectrum on a flat plane instead of on the Rowland circle (see Fig. 3.8).

The spectrum is recorded on a CCD camera. To increase the flux density at the detector plane, a cylindrical gold mirror is set at glancing angle in front of the diffraction grating. The use of the cylindrical mirror in conjunction with the grating allows for both spatial imaging and spectral dispersion. For many targets imaged, a double spectrum could be seen-one originating from the target rear plume, and one from the front plasma plume. The XUV flat-field spectrometer will also be discussed in more detail in Chapter 4.

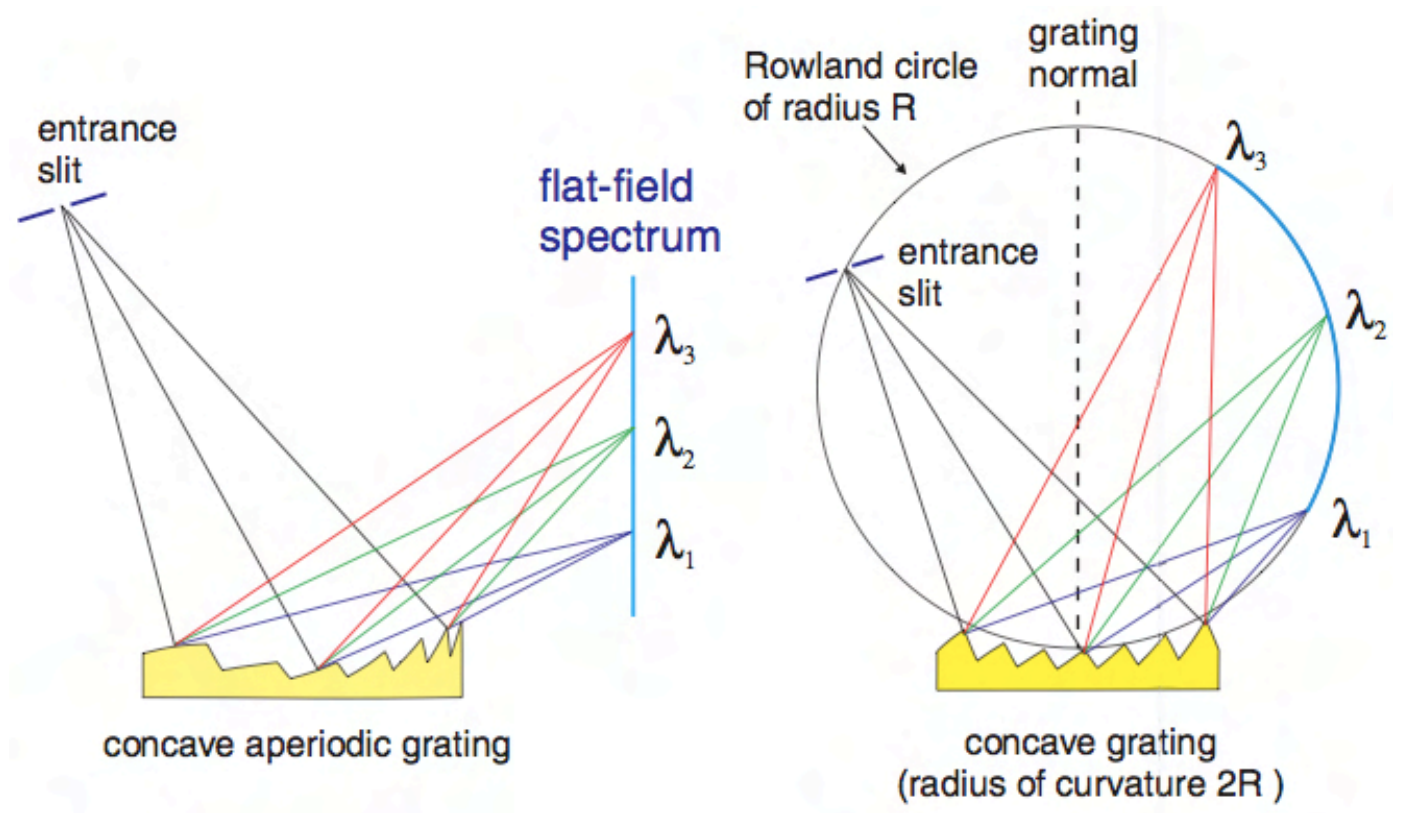

Figure 3.8: The use of a concave aperiodic grating allows the spectrum to be recorded on a flat plane, rather than on a curved surface matching the Rowland circle when simply using a concave grating. Image courtesy of M. Y. Shen. 


\subsubsection{3 $\mathrm{Cu} \mathrm{K} \alpha$ Imager}

One of the main goals of current Fast Ignition research is to characterize the flux of electrons created by laser irradiation within a solid target. Hot electrons originating in the critical density region of the plasma transport into the solid, and interact via electron impact ionization to produce a characteristic line spectrum. Given the high electron energies of interest, the x-ray emission occurring through $\mathrm{K}$-shell fluorescence is often used as a diagnostic for the hot electron number. If a K-shell electron is knocked out, the inner shell vacancy is rapidly filled by an Lor M-shell electron, producing $\mathrm{K} \alpha$ or $\mathrm{K} \beta$ radiation. Fig. 3.9 displays the energy diagram for nonionized copper.

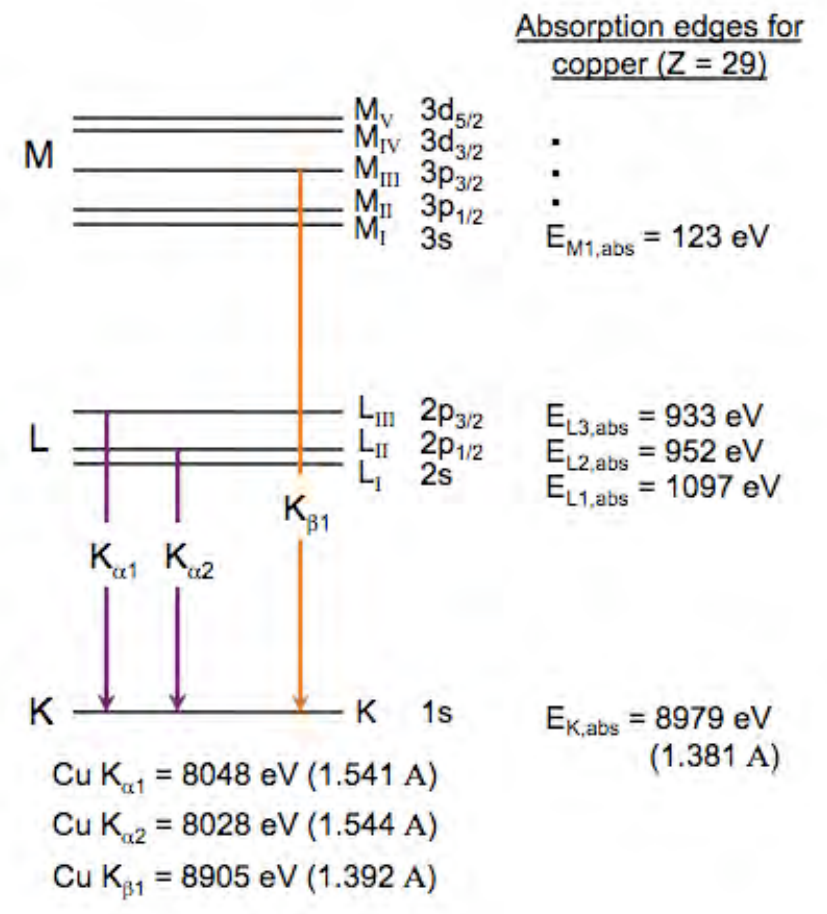

Figure 3.9: The energy level diagram for copper $(\mathrm{Z}=29)$. $\mathrm{K} \alpha$ radiation occurs through a $n=2$ to $n=1$ transition, while $\mathrm{K} \beta$ represents $n=3$ to $n=1$ ).

The probability of such transitions and the subsequent emission of a K-shell photon can be given by K-shell ionization cross sections by electron impact. The K-shell ionization cross section for copper as a function of overvoltage (incident 
electron energy / ionization energy of electrons in the $\mathrm{K}$ shell), as taken from empirical expressions described by Hombourger[64] is shown in Fig. 3.10. As can be seen, the cross-section peaks at approximately $3 \times$ the ionization energy, and evens out at higher energies. Therefore, K-shell (and therefore, $\mathrm{K} \alpha$ ) emission is more sensitive to electrons at relatively lower energies (a few ten $\mathrm{keV}$ to few hundred $\mathrm{keV})$.

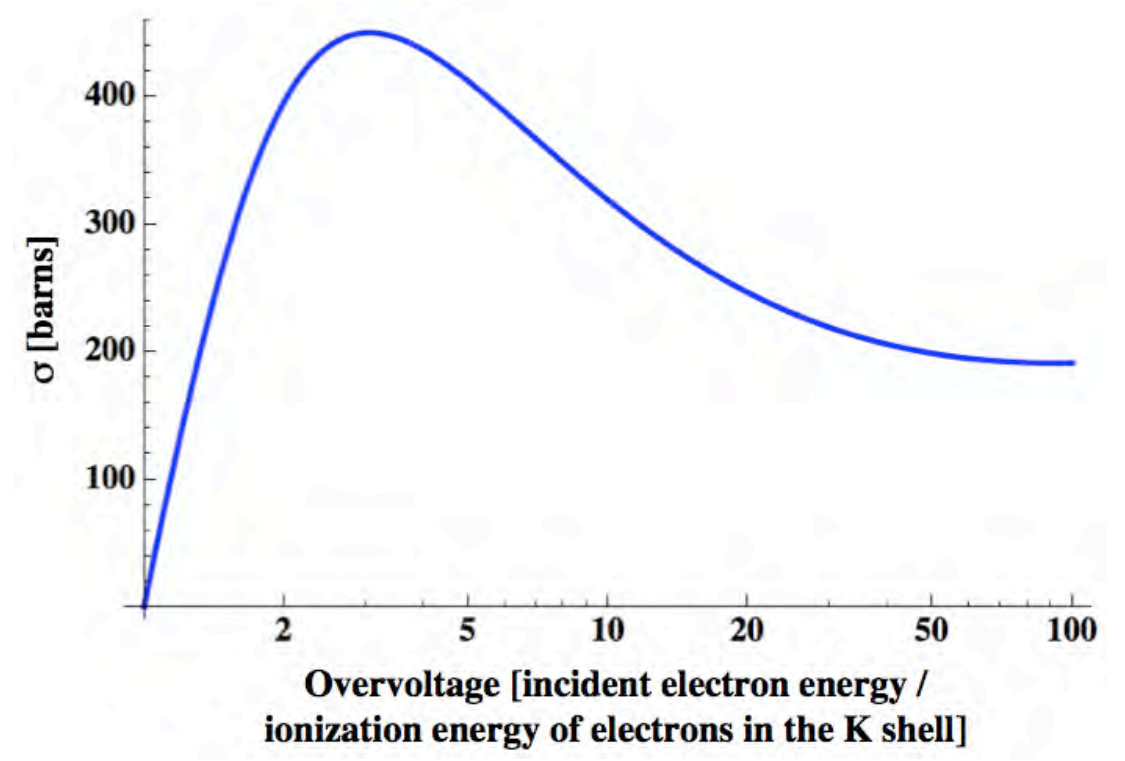

Figure 3.10: The K-shell ionization cross section for copper as a function of overvoltage. The curve is a fit to empirical data as described by Hombourger[64].

Because these $\mathrm{K} \alpha$ photons are predominantly created in the binary collisions of the hot electrons with the atoms of the irradiated material, they serve as a useful means to infer the number of hot electrons. The emission is isotropic and shortlived ( order of the laser pulse). Therefore, within an experiment, simultaneous diagnostics will be dedicated to detecting and recording the $\mathrm{K} \alpha$ emission. For experiments described within this thesis, copper is commonly used within the targets being irradiated, and so diagnostics such as the $\mathrm{Cu} \mathrm{K} \alpha$ Imager, the HOPG, and the Single Hit CCD are tuned to $8048 \mathrm{eV}$, the energy that cold $\mathrm{Cu} \mathrm{K} \alpha$ occurs at.

The $\mathrm{Cu} \mathrm{K} \alpha$ Bragg reflection crystal imager[65], consisting of a $\mathrm{SiO}_{2} 211$ quartz 


\section{Spherically bent quartz (211)}

2d: $3.082 \AA$,

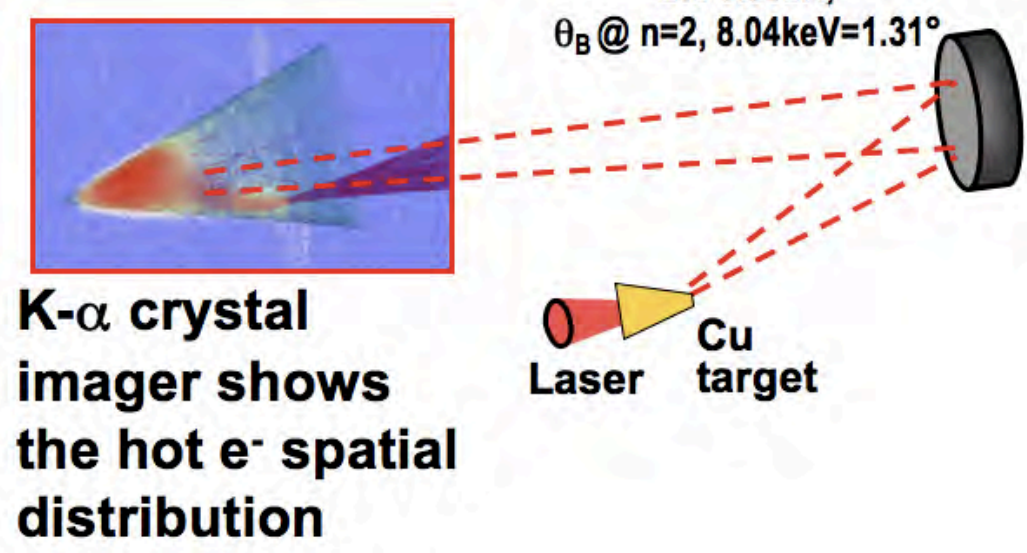

Figure 3.11: Schematic of the experimental setup of a $\mathrm{Cu} \mathrm{K} \alpha$ Imager.

crystal, gives 2-D time-integrated spatially resolved information of the hot electron flux by detecting $\mathrm{x}$-ray radiation within a narrow bandwidth around the $\mathrm{Cu} \mathrm{K} \alpha$ photon energy of $8048 \mathrm{eV}$. The imaging properties of a spherically bent crystal are similar to those of a spherical mirror, and thus can be understood with simple geometric optics.

One of the main limitations of using spherical optics is that, although they can provide high spatial resolution over large field of views, the angle of incidence must be near normal to minimize astigmatism. In the case of Bragg reflecting crystals, the $d$ spacing (interplanar spacing of reflecting surfaces) must be chosen to reflect the wavelength of choice, according to the Bragg relation,

$$
n \lambda=2 d \sin \theta
$$

while allowing $\theta$ to be within just a few degrees of normal. The $\mathrm{Cu} \mathrm{K} \alpha$ crystals used in the experiments here had $2 d$ spacings of $3.082 \stackrel{A}{A}$, and were operated at $1.3^{\circ}$ off axis in second diffraction order.

The extent to which a spherical imager can resolve the separation between two objects is called the spatial resolution, and is determined by a combination of the diffraction limited spot, the spherical aberration and astigmatism. The diffraction 
limited spot made by a curved optic can be described as,

$$
\sigma_{d l}=1.22 \lambda f / M=1.22 \lambda f_{0},
$$

where $\lambda$ is the wavelength of light being imaged, $M$ is the magnification of the system, $f=L / D$ is the f-number associated with the optic (where $L$ is the focal length and $\mathrm{D}$ is the diameter of the optic), and $f_{0}=L_{0} / D$ is the f-number associated with the object (and $L_{0}$ is the object to optic distance). In cases where an aperture is used in front of the optic, D will be the aperture diameter.

Spherical aberrations will occur due to light rays, depending on where on they optic they are reflected, being focused to a slightly different spot. The larger the aperture size, the larger the spherical aberration, or blurring of the image. The spherical aberration is,

$$
\sigma_{s a}=\frac{1}{2 M}\left[\left(L_{i}-L\right) \tan \phi-\frac{D}{2}\right]
$$

with,

$$
\phi=2 \arcsin \left(\frac{D}{2 R}\right)-\arctan \left(\frac{D}{2 L_{0}-2 L}\right)
$$

and,

$$
L=R-\frac{\lambda}{2 \arcsin \left(\frac{\lambda}{2 R}\right)} .
$$

Astigmatism for a spherical optic is due to the radius of curvature, $R$ of the optic having slightly different focusing in the meridional and sagittal planes. Then, instead of focusing to one single point, rays will focus to a circular region, called the circle of least confusion. The diameter of this circle composes the $\sigma_{a s}$,

$$
\sigma_{a s}=D\left(1-\sin \theta_{B}\right) \frac{M+1}{M} .
$$

Each of these factors that reduces that spatial resolution can be combined in quadrature to give the total image resolution as,

$$
\sigma_{T}=\sqrt{\sigma_{d l}^{2}+\sigma_{s a}^{2}+\sigma_{a s}^{2}}
$$

In the RAL (Titan) configuration, the crystal used had a radius of curvature of $38 \mathrm{~cm}(50 \mathrm{~cm})$, placed $20 \mathrm{~cm}(21.5 \mathrm{~cm})$ from the target. The magnification was 
$15 \times(7.75 \times)$, and in both cases, the aperture in front of the crystal was $16 \mathrm{~cm}$ in diameter. This means that the RAL imager had a theoretical total resolution of approximately $6 \mu \mathrm{m}$, and the Titan imager had a resolution of approximately 5 $\mu \mathrm{m}$.

Imaging was done onto Fujifilm BAS-SR image plates at RAL, and a Princeton Instruments PI-SX1300 CCD camera at Titan.

While the narrow bandwidth of this diagnostic allows for monochromatic imaging, this is also one of its limitations: The $\mathrm{K} \alpha$ spectral line shifts and broadens with increasing target temperature, and so, for sufficient temperature increases, shifts off the narrow bandwidth of the crystal [66]. The collection efficiency of the $\mathrm{Cu} \mathrm{K} \alpha$ imager is thus dependent on the temperature of the target, as shown in Fig. 3.12, and corrections need to be made for the loss of signal due to it.

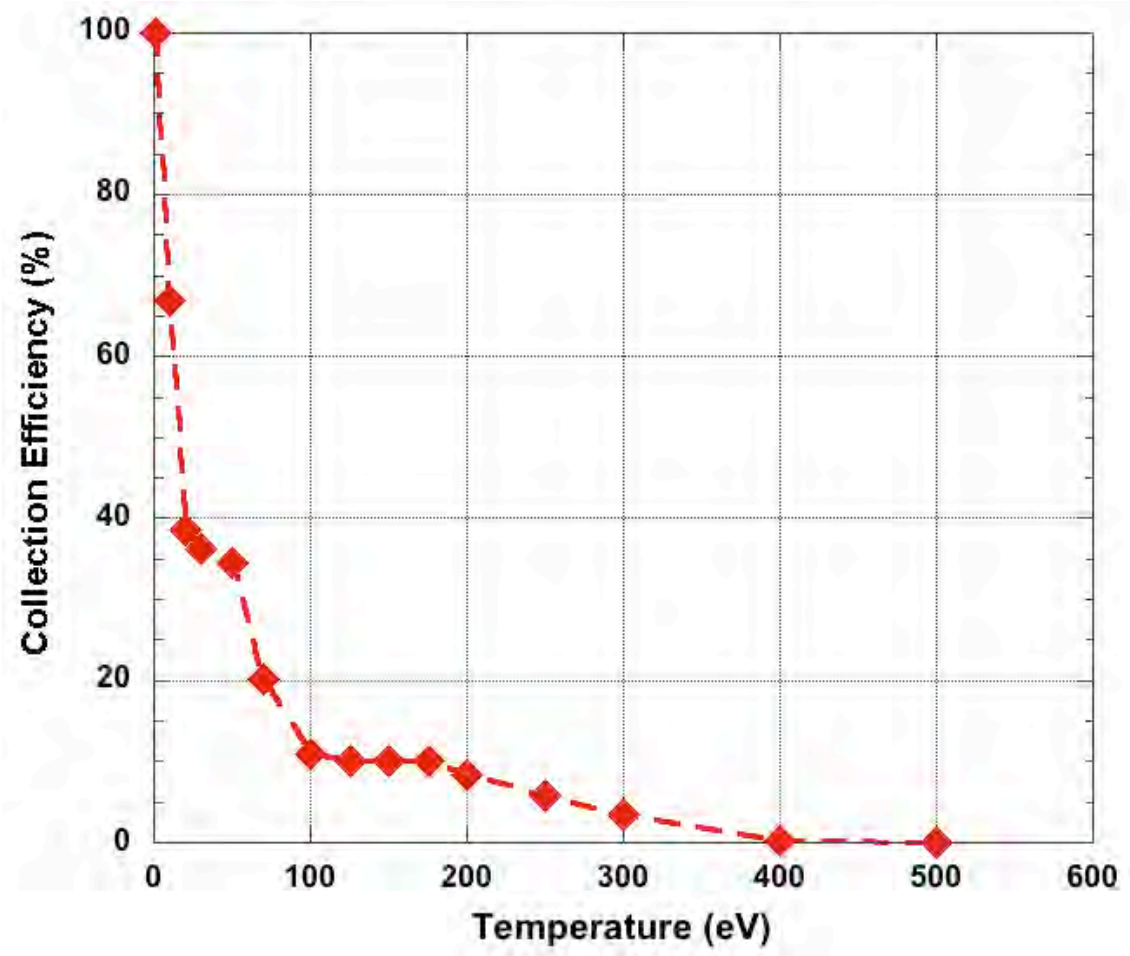

Figure 3.13: The collection efficiency of the $\mathrm{Cu} \mathrm{K} \alpha$ decreases as a function of temperature due to the shifting and broadening of the $\mathrm{K} \alpha$ line out of the effective bandwidth of the imager diagnostic. Data points are derived by evaluating the ratio of area under the $\mathrm{Cu} \mathrm{K} \alpha$ line emission bounded by the aperture energy bandwidth of the diagnostic. Data courtesy of K. Akli. 


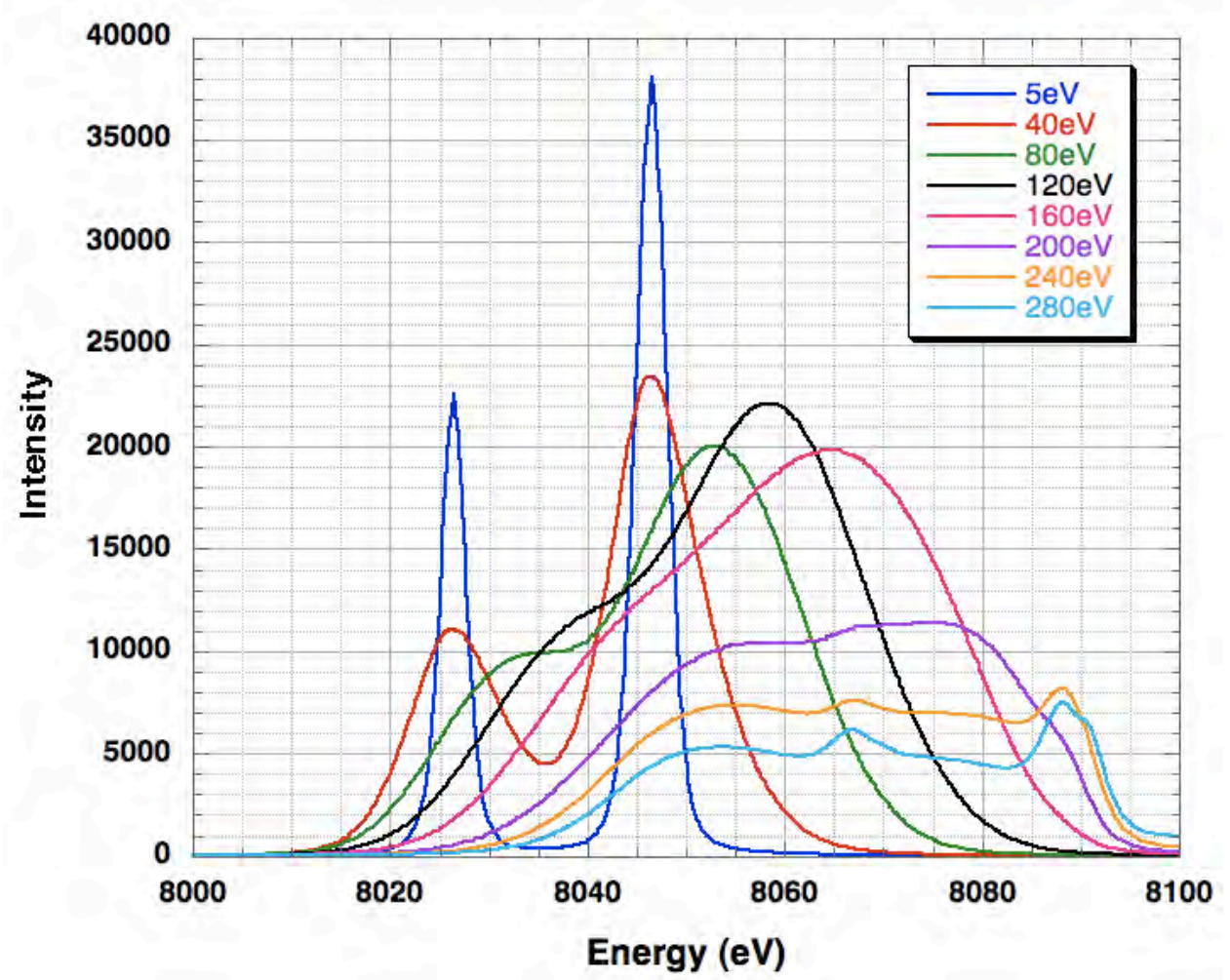

Figure 3.12: The shift of the $\mathrm{Cu} \mathrm{K} \alpha$ line as a function of temperature. Spectra as predicted from the 0-D collisional radiative code FLYCHK. 


\subsubsection{Highly Oriented Pyrolytic Graphite (HOPG) Spectrometer}

The HOPG, or Highly Oriented Pyrolytic Graphite, Spectrometer consists of a thin film crystal with a random distribution of scattering planes which allows for highly efficient spectral resolution of x-ray lines emitted in a laser-matter interaction $[67,68]$. These crystals operate on the Bragg relation:

$$
n \lambda=2 d \sin \theta_{B}
$$

where $\mathrm{n}$ is the diffraction order, $\lambda$ is the wavelength of the radiation, $\mathrm{d}$ is the separation between the mosaic crystal planes of the crystal, and $\theta_{B}$ is the Bragg angle.

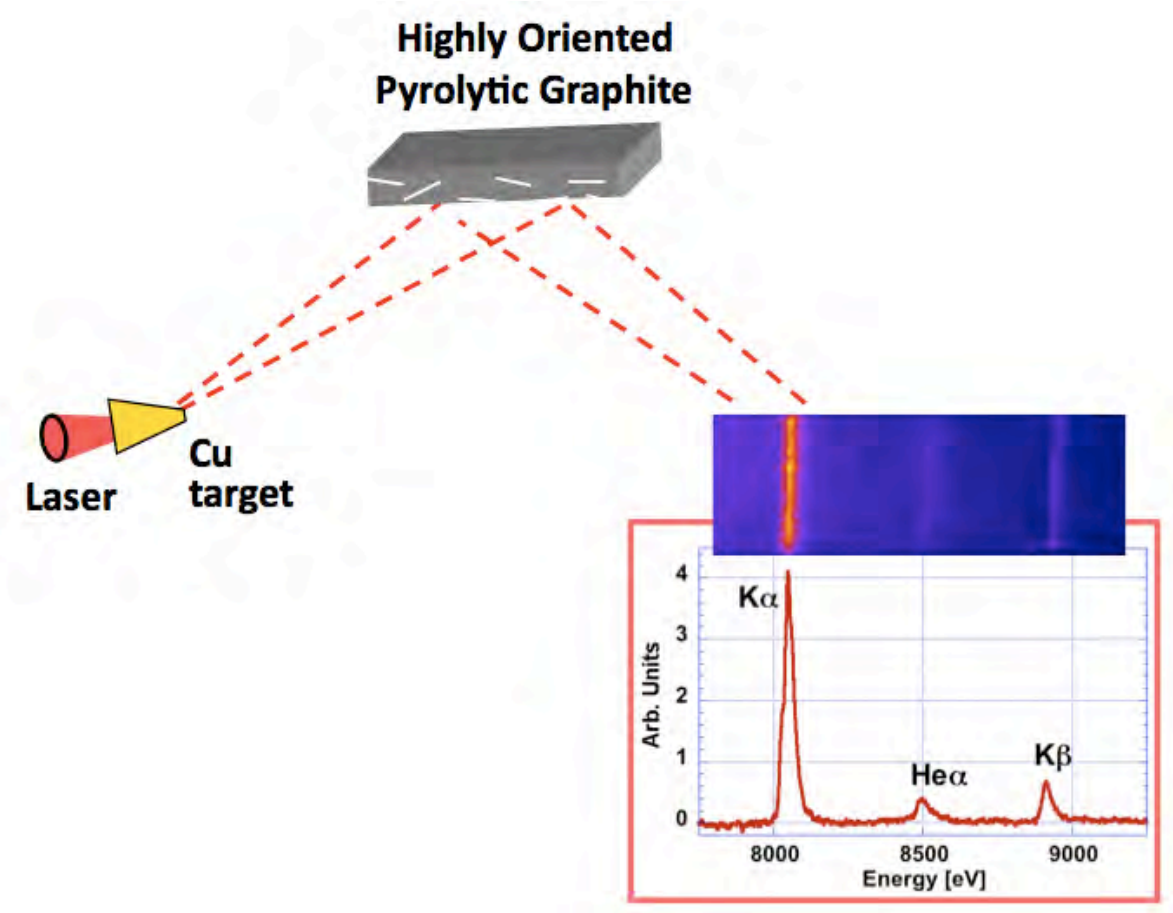

Figure 3.14: Schematic of the experimental setup of a HOPG crystal.

The natural mosaicity of these crystals (i.e., slight misalignment of adjacent lattices to give an angular distribution of the crystallites) allows a spectrum of $\mathrm{x}$-rays emitted by a point source to always find a scattering plane in the crystal where it will satisfy the Bragg condition. Therefore, the mosaicity of the HOPG 
crystal allows for an increase of integral reflectivity (over ideal crystals), and for mosaic focusing (see Fig. 3.15).

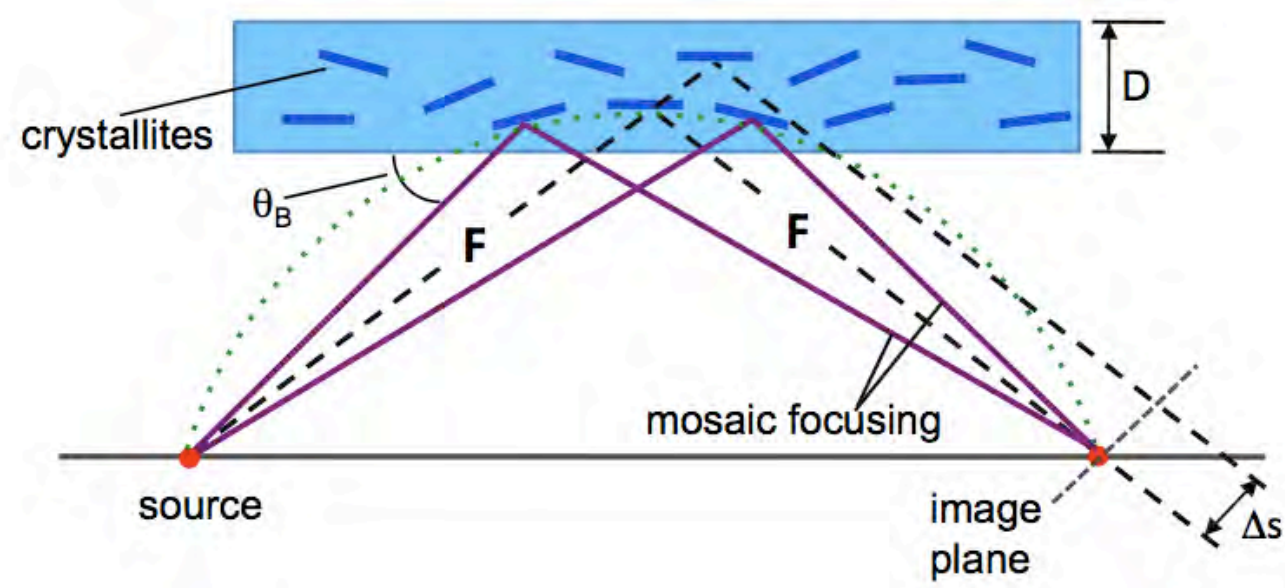

Figure 3.15: The intrinsic mosaicity of the HOPG crystal allows for rays emitted by a point source to be focused onto a plane in the image plane if the crystallites are lying on a Rowland circle. This mosaic focusing occurs in the 1:1 magnification geometry, when the focal length $F$ between the source and the crystal, and the crystal and the image plane are equal. When rays are reflected off multiple crystallites at different depths, a focusing error giving the x-ray line width $\Delta s$ will occur.

The idea behind mosaic focusing, or parafocusing, is that when the distance between the point source and the crystal, and the crystal and the image plane are equal (a 1:1 magnification geometry), and the crystallites of the HOPG are lying on a Rowland circle, monochromatic light will be focused in the diffraction plane. It would be as if a set of the crystallites were composing a curved surface, like a spherical mirror, to focus the x-rays. When rays of a different energy are incident on the HOPG crystal, they will find crystallites that satisfy their Bragg condition, and these rays will be focused to a different point, in a plane perpendicular to the diffraction plane. Then, by placing the imaging substrate along this perpendicular plane, a full set of spectrally resolved x-rays can be recorded[69].

The mosaicity of the graphite crystal means that there will be some intrinsic width of the Bragg reflection due to the particle size and strain on the particles. 
This intrinsic width of the Bragg reflection is given by

$$
\frac{\Delta E}{E}=\frac{\Delta \theta}{\tan \left(\theta_{B}\right)}
$$

and represents the upper limit for the energy resolution, up to $5.35 \times 10^{-4}$ in first order reflection. However, the crystal thickness can also affect the energy resolution, as shown by the wide dotted line if Fig. 3.15. As an incoming x-ray beam searches for a crystal plane it can diffract off of, it may find a plane deeper within the crystal beyond the surface. Reflection off this plane will contribute to spatial smearing of the imaged signal at the imaging plane. By using a thinner HOPG crystal, this penetration depth broadening can be improved, but at the cost of intensity. Actual measured values of $\Delta E / E$ then tend to lie in the range of $1-3$ $\times 10^{-3}[70]$.

Once a spectrum is recorded at the image plane, the HOPG data can be analyzed to retrieve relative line intensities, total counts of a particular line radiation, and shift and broadening of a particular line due to heating of the plasma. The HOPG spectrometer is an essential diagnostic to run in conjunction with the $\mathrm{K} \alpha$ imager because its broad bandwidth consistently allows all the $\mathrm{K} \alpha$ line radiation to be collected, and therefore is not sensitive to shifting of the line due to temperature or density effects.

The HOPG crystals used in the experiments discussed here were 2 mm-thick, ZYA crystals manufactured by GE Advanced Ceramics. They had a mosaic spread $(\gamma)$ of $0.4^{\circ}$, where $\gamma$ is defined as the full width half maximum of a Gaussian distribution of crystal plane orientations off the normal axis to the surface. The $d$ spacing, or separation between the mosaic crystal planes was $d=0.3354 \mathrm{~nm}$,

By changing the angle of the crystal, the orientations of the crystal planes can be changed to reflect over a different spectral bandwidth. In most cases, the $\mathrm{Cu}$ $\mathrm{K} \alpha$ line emission was the target radiation, and so the crystal was set to $13.4^{\circ}$ to reflect x-rays over a $3.5 \mathrm{keV}$ spectral bandwidth centered about $8048 \mathrm{eV}$. Images were recorded on Fujifilm BAS-SR or MS image plates. 


\subsubsection{Single Photon Counting CCD}

The Single Photon Counting CCD, or Single Hit CCD (SHCCD) is a chargecoupled device (CCD) camera operating as an x-ray spectrometer by placing the camera sufficiently far away and filtered in such a way that no more than one x-ray photon is incident upon every pixel of the camera. Below $100 \mathrm{keV}$, most photons will deposit their energy in one pixel. In this mode, then, the value of each pixel will be proportional to the energy deposited by the photon[71, 72]. By taking a histogram of the pixel readout values, an approximation can be made of the x-ray spectrum. This technique gives an absolute measure of K-shell radiation emission.

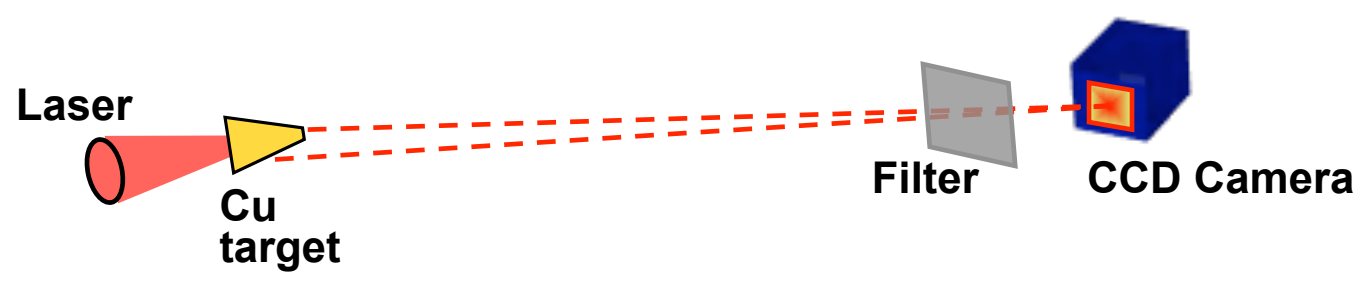

Figure 3.16: Schematic of the experimental setup of a Single Photon Counting CCD

The CCD camera employed was a Spectral Instruments Series 800 Camerea using a $2048 \times 2048$-pixel, backthinned CCD chip with a pixel size of $13.5 \mu \mathrm{m}$. In most experiments, the camera is placed as far away from the interaction as reasonable, while still having a line-of-sight to the target chamber center. This distance is generally on the order of several meters, to allow the solid angle to be small enough to limit the number of photons that will hit the CCD chip. However, it is preferable to have that entire propagation span be composed of vacuum, rather than air, because the attenuation length of an $8048 \mathrm{eV}$ photon is approximately 50 $\mathrm{cm}$ in air, and using vacuum will help maintain a good signal to noise level. If it is not possible to fully achieve this span while having the camera directly mounted on the main chamber, a vacuum tube can be employed to displace the air gap between the main chamber and an externally pumped chamber where the camera resides. Also, it is good practice to surround the CCD camera with lead shielding to minimize the radiation background[71]. 
In the analysis of a SHCCD readout[73], the number of photons detected by the SHCCD is a function of the number of hits on the chip $(H)$, the solid angle of the chip $(\Omega)$, the filtering of the camera $\left(f_{T}\right)$, the chip efficiency $\left(\epsilon_{e f f}\right)$ as a function of the chip coverage $(\psi)$, and the K $\alpha$ energy $(\varepsilon)$, in the relation,

$$
\frac{K_{\alpha}}{\Omega}=\frac{H}{\Omega \times f_{T} \times \epsilon_{e f f}(\varepsilon, \psi)} .
$$

Chip coverage, $\psi$, is the number of CCD pixels with counts over some threshold value that differentiates actual signal from background. To determine $H$, an algorithm is run to find "single hits,", or instances in which a photon deposits all of its energy within an isolated pixel of the chip (rather than split events where the photon energy is deposited over two or more pixels). The energy within pixels that are counted as hits can then be multiplied by the work function of the chip material and the gain of the camera to give an energy spectrum. The chip efficiency, $\epsilon_{\text {eff }}$, which is a function of the photon energy (in our case, we are interested in the $\mathrm{K} \alpha$ photons) and the chip coverage, has been determined through extensive CCD calibrations[72]. The other variables in the equation will be a function of the individual experiment or shot setup.

\subsubsection{Cross-Calibration of the $\mathrm{Cu} \mathrm{K} \alpha$ Diagnostics}

The idea behind the redundancies of the numerous $\mathrm{Cu} \mathrm{K} \alpha$ diagnostics is to derive an absolute number of $\mathrm{K} \alpha$ photons for any one data shot. The single photon counting technique, by virtue of distance and careful filtering ensures a controlled and countable number of K $\alpha$ photons. However, it is prone to large shot-to-shot variations, often on the order of a factor of two or more, due simply to statistical variation and random errors (chip coverage, fluctuating noise levels, etc). The HOPG spectrometer, on the other hand, is far more precise, but its recorded values are generally in some arbitrary unit, which is a function of the image plate scanner.

A cross-calibration then needs to be done using a number of simple targets (often thin $\mathrm{Cu}$ foils), where the number of $\mathrm{K} \alpha$ photons as counted by the SHCCD is plotted against the integrated yield from the HOPG. View angles, opacity cor- 
rections, and solid angle of coverage have to be taken into account for each diagnostic. The relation between the yields from each diagnostic should be linear, with a weighting at $(0,0)(0$ counts on the HOPG should correspond to 0 counts on the SHCCD). The slope of the best fit line of the HOPG versus the SHCCD can then be used as the conversion factor to apply to other HOPG measurements within the experiment on various target configurations to get an absolute $\mathrm{K} \alpha$ yield.

This number can then also be applied to the $\mathrm{K} \alpha$ measurements made with the 2-D $\mathrm{Cu} \mathrm{K \alpha}$ imager. Because, as mentioned above, the 2-D imager is sensitive to the shifting of the $\mathrm{K} \alpha$ line, the absolute yield of the $\mathrm{K} \alpha$ as determined by the cross-calibration is a key value that the signal on the imager must be renormalized to following corrections to account for line shifting with temperature.

Because these cross-calibrations can be sensitive to the individual diagnostic, diagnostic setup, and laser conditions, it is worthwhile to repeat this process should there be an uncertainty in the total $\mathrm{K} \alpha$ yield. More details will be found in the descriptions of the analyses for the nail-wire and cone-wires in subsequent chapters. 


\section{Chapter 4}

Measurement of Electron-Heated

\section{Target Temperatures using}

\section{Extreme Ultraviolet Imaging and}

\section{Spectroscopy}

\subsection{Theory of Extreme Ultraviolet Radiation}

In the development of fast ignition[6], it is important to understand energy deposition by intense laser-generated relativistic electrons in a solid target or dense plasma. In this process, a picosecond laser pulse launches the electrons into the target where they cause both heating and radiation. This heating can be measured by a number of different diagnostic instruments that selectively capture optical Planckian radiation[74], electron excited K $\alpha$ emission[75], or soft x-ray to hard x-ray emission[76, 67].

Although the plasmas created in an intense laser-solid interaction are rapidly changing, a large fraction of the energy will still be contained within a near-thermal distribution. By making the limiting case assumption that a material is, in fact, in thermodynamic equilibrium with its surroundings, then the blackbody radiation emitted will be characteristic of a single temperature, $T$. 
Planck[77] showed that if it is assumed that radiation is emitted in discrete quantas of energy, with energy proportional to frequency, then the spectral brightness of blackbody radiation is given as[78]

$$
B_{\Delta} \omega / \omega=3.146 \times 10^{20}\left(\frac{\kappa T}{e V}\right)^{3} \frac{(\hbar \omega / \kappa T)^{3}}{\left(e^{\hbar \omega / \kappa T}-1\right)} \frac{\text { photons } / \mathrm{s}}{\mathrm{mm}^{2} \cdot s r \cdot(\Delta \omega / \omega)} .
$$

The spectral brightness has a dependence of the form $x^{3} /\left(\mathrm{e}^{x}-1\right)$, where $x=\hbar \omega / \kappa T$. This Planckian function has a max value of 1.421 at $x=2.822$, so that the peak spectral brightness occurs at a photon energy

$$
\left.\hbar \omega\right|_{p k}=2.822 \kappa T \text {. }
$$

The Planckian XUV emission studied here crests in a few picoseconds then decays as the target cools[60]. Because of the rapid variation of radiation intensity with temperature and the very short absorption length characteristic of emission in the XUV region (5 - $40 \mathrm{~nm}, 30-250 \mathrm{eV}$ ), time-integrated imaging, as well as spectroscopic resolution of the radiation offers an excellent tool to determine plasma temperatures.

Previously, $\mathrm{Gu}$ et al.[60] used a $68 \mathrm{eV}$ XUV imaging diagnostic to measure short pulse laser heating of aluminum foils of various thicknesses. Calibration results for the various components of the diagnostic and methodology for calculating temperatures based on the XUV images were presented. It was determined that an electron heated $50 \mu \mathrm{m}$ thick planar $\mathrm{Al}$ foil reached a temperature of $22 \mathrm{eV}$ on the target rear surface. This thesis work significantly advances the work by $\mathrm{Gu}$, et al. to include temperature measurements from $256 \mathrm{eV}$ imaging and makes a comparison of temperature at two photon energies (i.e., 68 and $256 \mathrm{eV}$ ).

Emission at these two energies is significantly different because different surfaces are detected. The plasma expanding from the hot target is significantly more opaque at $68 \mathrm{eV}$ than $256 \mathrm{eV}$. At the lower photon energy the afterglow is longer and that emission incorporates more hydrodynamic expansion. This extended glow allows for bright images and imaging of expansion plumes, but also leads to blurring of the initial heated state. In contrast, the duration of the emission is much shorter at $256 \mathrm{eV}$, and so in essence, "freezes the time-integrated emission 


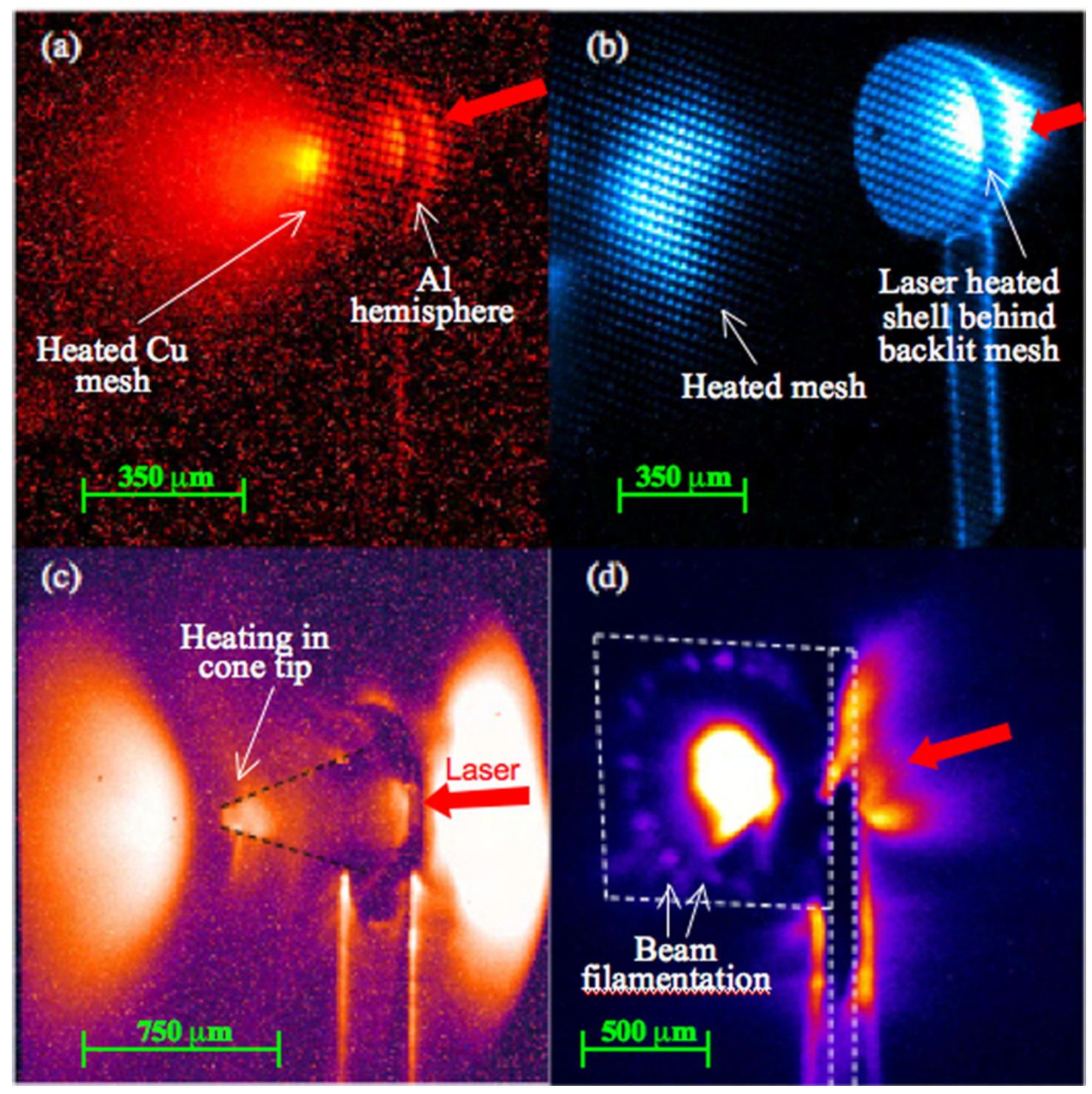

Figure 4.1: (a) A $256 \mathrm{eV}$ image of a half sphere with a grid $228 \mu \mathrm{m}$ behind it. (b) A $68 \mathrm{eV}$ image of a half sphere with a grid $804 \mu \mathrm{m}$ behind it. (c) A $68 \mathrm{eV}$ image of a copper cone. (d) A $68 \mathrm{eV}$ image of a $10 \mu m \mathrm{CD} / 5 \mu m \mathrm{Al} / 10 \mu m \mathrm{CD}$ sandwich. 
by capturing it before the occurrence of significant hydrodynamic expansion of the exploding target. Fig. 4.1 shows several examples of XUV images of different targets irradiated with a short-pulse laser. Fig. 4.1(a) shows a $256 \mathrm{eV}$ image of a half sphere with a mesh grid $228 \mu \mathrm{m}$ behind it, (b) is a $68 \mathrm{eV}$ image of a half sphere with a grid $804 \mu \mathrm{m}$ behind it, (c) is a $68 \mathrm{eV}$ image of a copper cone, and (d) is a $68 \mathrm{eV}$ image of a $10 \mu \mathrm{m} \mathrm{CD} / 5 \mu \mathrm{m} \mathrm{Al} / 10 \mu \mathrm{m}$ CD sandwich planar target. Very clearly seen in the images is the laser interaction region, the varying thermal distribution of electrons based on target geometry, the blow-off plasma plumes at both the targets' front and rear, and in some cases, filamentation of the electron beams.

In this chapter, it is shown that temperatures for a range of heating of thin foil targets can be determined and verified independently by both XUV imagers. The temperature range in which both imagers overlap to accurately establish a target temperature is discussed, and evidence is presented showing that in order to use this diagnostic quantitatively, extreme care must be taken to ensure that angles of incidence on the multilayer XUV mirrors are within the required tolerances. Finally, peak rear surface temperatures derived from the two imagers are compared against an average temperature of the rear surface plasma plume deduced by performing spectroscopy in the XUV range using a flat field spectrometer (FFS).

\subsection{The XUV Imaging Diagnostic}

\subsubsection{Diagnostic Geometry}

The XUV imaging diagnostic collects light within a solid angle determined by an aperture and relays this to a charge-coupled device (CCD) camera via two multilayer XUV mirrors that dictate the energy bandwidth of the light imaged. Fig. 4.2 shows a schematic of the XUV imaging diagnostic setup. Two independent channels of the XUV diagnostic were employed: one optimized for $68 \mathrm{eV}(18.2 \mathrm{~nm})$ light and the other for $256 \mathrm{eV}(4.8 \mathrm{~nm})$.

In each XUV channel, the first mirror is spherical and images the target, while the second, a flat mirror, turns the beam through $90^{\circ}$. The spherical-plane mirror 


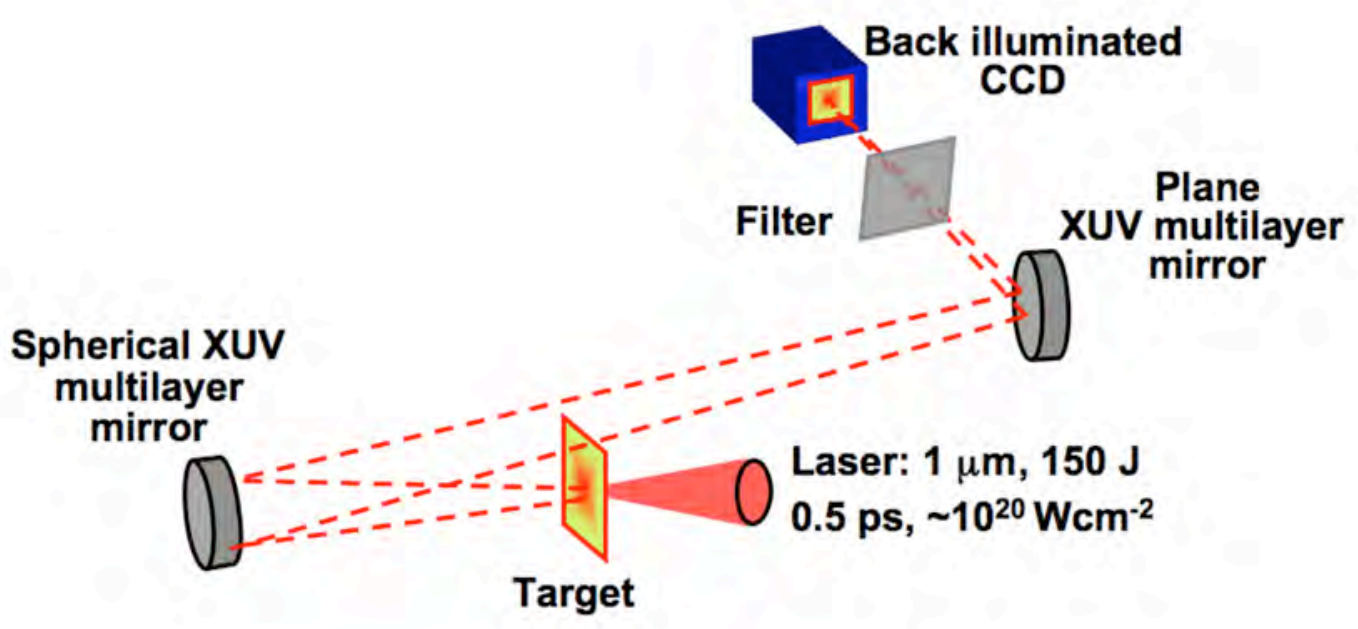

Figure 4.2: Schematic of XUV imaging diagnostic setup.

pairs are manufactured by T. Barbee of LLNL to have matching spectral peaks. The $68 \mathrm{eV}$ mirrors are a $\mathrm{Mo}_{2} \mathrm{C} / \mathrm{Si}$ multilayer, with the focusing (spherical) mirror having 21 pairs of layers alternating between $\mathrm{Mo}_{2} \mathrm{C}$ and $\mathrm{Si}$, for a total bi-layer period of $96 \stackrel{\circ}{A}$, while the associated plane mirror has 11 pairs of the same layers at a bi-layer period of $142 \stackrel{\AA}{A}$. The $256 \mathrm{eV}$ mirrors are a C/WC/Monel/W formula, with the focusing mirror composed of 100 layer pairs of $24 \stackrel{A}{A}$ bi-layer periods and the plane mirror composed of 81 layers of $34-35.5 \stackrel{\circ}{A}$.[79]

The use of two optics with large-angle bends in each imaging line allows the detector to be screened from stray radiation. The spherical mirrors, which are set at near-normal incidence to minimize spherical aberrations, have a radius of curvature of $0.5 \mathrm{~m}$, and are placed approximately $0.27 \mathrm{~m}$ away from the target. The reflected image is then projected onto the second multilayer (the plane mirror) at a path length of approximately $2.15 \mathrm{~m}$, where it is deflected $90^{\circ}$, then goes through a filter which blocks IR to UV radiation due to self-emission from the target (which is also reflected from the mirrors), before reaching the CCD.

The total magnification of the imaging system is approximately 11, and a $6 \mathrm{~mm}$ aperture in front of the spherical mirror gives a collection solid angle of $5.2 \times 10^{-4}$ sr. Considering the off-axis and spherical aberrations, the resolution of the system 


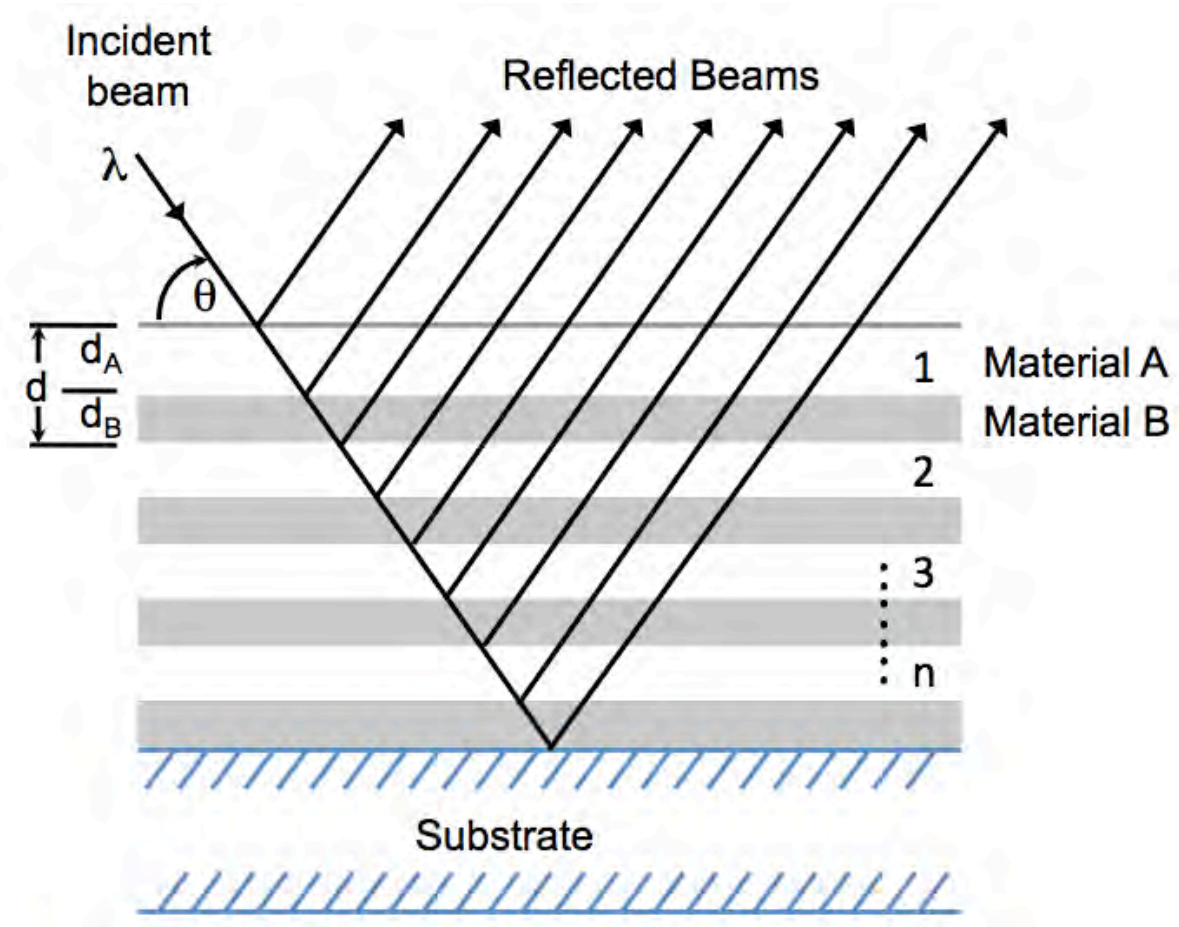

Figure 4.3: Schematic of multilayer reflector of $n$ bilayer pairs. The parameters $\lambda$, $\theta$, and $d$ are chosen to satisfy the Bragg equation, and the relative thicknesses of the high- and low- $\mathrm{Z}$ materials are chosen to optimize reflectivity.

is approximately $9 \mu \mathrm{m}$.

The thin aluminum and polyimide filter is designed to eliminate any imaging of radiation in the ultraviolet, visible and infrared. A variety of these filters, which range in thickness from $1000-2000 \AA$ of aluminum coupled with $2100-2500 \AA$ of polyimide, effectively operate as ND 0.5 - 1 filters at $256 \mathrm{eV}$, and ND 0.7 - 3 filters at $68 \mathrm{eV}$. Due to the necessarily thin $\mathrm{Al}$ layers required for efficient transmission of the XUV, there can be issues with residual transmission of thermal IR to UV radiation and electron induced transition radiation within the response bandwidth of the CCD and reflected by the mirrors. This is primarily a problem with the 256 eV XUV channel when XUV signal levels are low and near the detection threshold, which occurs with thicker targets. This sets a lower limit for useful signal which is slightly higher than that where the signal is undetectable in the background noise.

The same Princeton Instrument PI-SX: 1300 CCD cameras used and calibrated 
by $\mathrm{Gu}$ et al.[60] are used to record the images with a chip resolution of $20 \mu \mathrm{m}$. The quantum efficiency of these cameras is $42 \%$ for $68 \mathrm{eV}$ and $48 \%$ for $256 \mathrm{eV}$. All other values for the CCD used in the analysis are based on results from the $\mathrm{Gu}$ calibration.

\subsubsection{Calibration of Multilayer Mirrors}

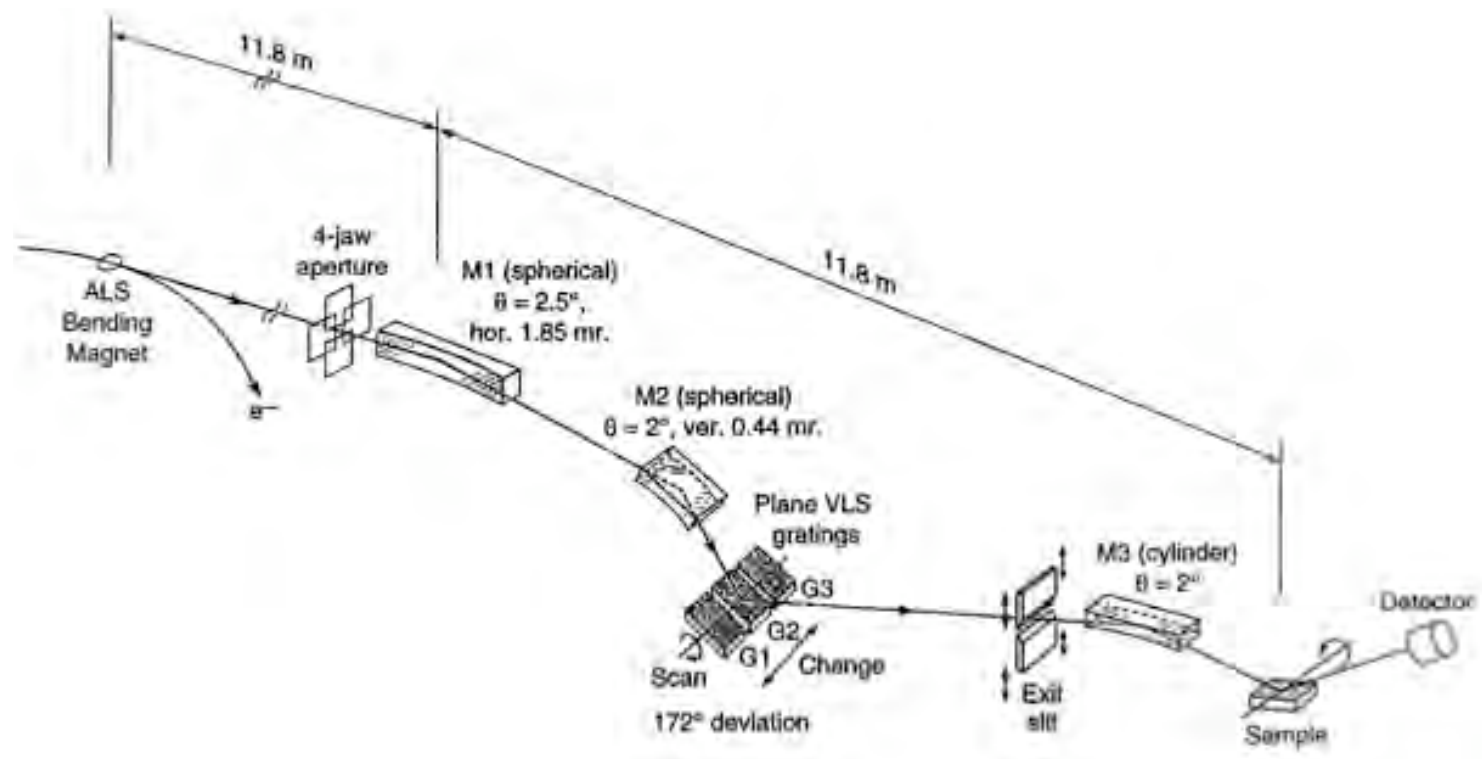

Figure 4.4: A schematic of the LBL Center for X-ray Optics Beamline 6.3.2 at the Advanced Light Source which was used for the calibration of the XUV multilayer mirrors. Figure taken from Underwood, Review of Scientific Instruments (1996)[80].

The XUV multilayer mirrors were calibrated at the Lawrence Berkeley National Laboratory Center for X-ray Optics Beamline 6.3.2 at the Advanced Light Source[80]. The beamline is optimized for the 50 - $1300 \mathrm{eV}$ energy range and is dedicated to EUV and soft x-ray reflectometry and scattering. See Fig. 4.4 for a schematic view of the beamline. The beamline utilizes a monochromator, a reflectometer, and refocusing mirrors to form a small spot on the sample (the multilayer mirror). A variable-line spaced plane grating monochromator scans the wavelength by rotating the grating with a fixed exit slit. The sample is mounted vertically in 
the reflectometer end-station and can be rotated about its vertical axis, as well as translated in the three orthogonal directions, with a position accuracy of $4 \mu \mathrm{m}$. A detector, on a rotating arm, measures the reflection off the mirror. The radiation from the beamline is completely $S$ polarized, whereas photons from the laser-driven foil are expected to be unpolarized. Thus for the $45^{\circ}$ reflections of the flat mirrors in the calibration, the measured reflectivity was halved in the plotted results to allow for the fact that reflectivity is zero (Brewsters angle) for $P$ polarization.

The reflectivity of the spherical mirrors was determined by scanning in photon energy at near-normal grazing incidence angles $\left(85^{\circ}, 83^{\circ}\right.$, and $75^{\circ}$, and hence inferring the reflectivity at $90^{\circ}$ ) to give plots of reflectivity as a function of energy at specific angles. Turning (flat) mirrors were irradiated at $43^{\circ}, 45^{\circ}$, and $47^{\circ}$ grazing incidences, also scanning in photon energies. (All angles are taken with respect to the mirror surface.) Fig. 4.5 depicts the reflectivities of the $68 \mathrm{eV}$ and $256 \mathrm{eV}$ spherical and turning mirrors as a function of energy for select grazing incidence angles. The shifts of peaks not plotted were found to follow the expected Bragg reflection scaling.

The calibrations show that the $68 \mathrm{eV}$ spherical and plane mirrors are fairly accommodating in the angle of incidence: an incident angle of anywhere from 83$90^{\circ}$ for the spherical mirror and $44-45^{\circ}$ for the plane mirror will reflect energies between $68-69 \mathrm{eV}$. The angle of setup for the spherical mirrors in the experiment was chosen to be $88^{\circ}$ grazing incidence, to limit off-axis aberrations and to closely coincide with the peak reflectivity of the turning mirror at $45^{\circ}$.

The angle of incidence of the $256 \mathrm{eV}$ spherical mirror is shown to be slightly more critical, where an incident angle of $90^{\circ}$ has a peak reflectivity at $261.8 \mathrm{eV}$, while shifting that incidence angle by $5^{\circ}$ to $85^{\circ}$ moves the reflectivity peak to 262.4 $\mathrm{eV}$. The $256 \mathrm{eV}$ plane mirror is found to be even more sensitive: a setup angle of $45^{\circ}$ peaks at $254.8 \mathrm{eV}$, but shifting the mirror only two degrees to a grazing incidence angle of $43^{\circ}$ pushes the peak to $263.8 \mathrm{eV}$. The angle of setup for the spherical mirror was chosen to be $88.5^{\circ}$, and in order to achieve maximum overlap of the bandwidth of the pair of multilayer mirrors, the turning mirror was set at $43.5^{\circ}$, rather than the nominal $45^{\circ}$. The markedly higher sensitivity of the 256 


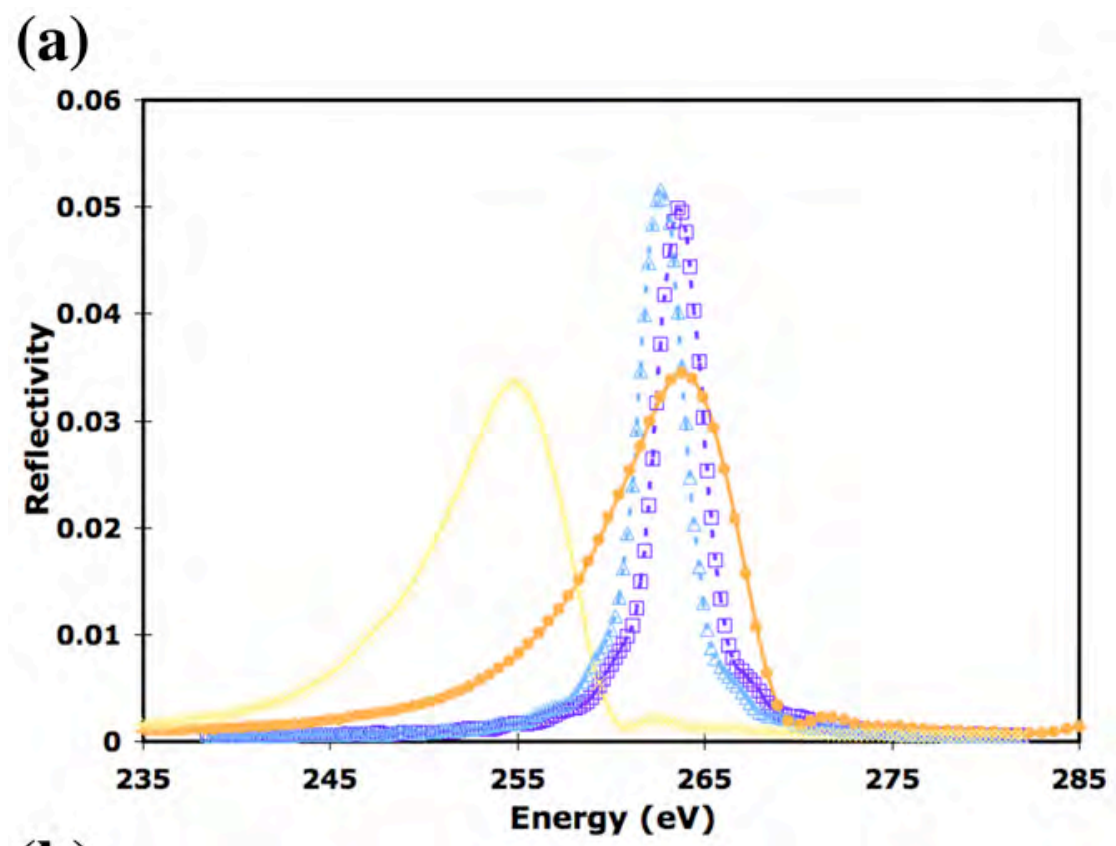

(b)

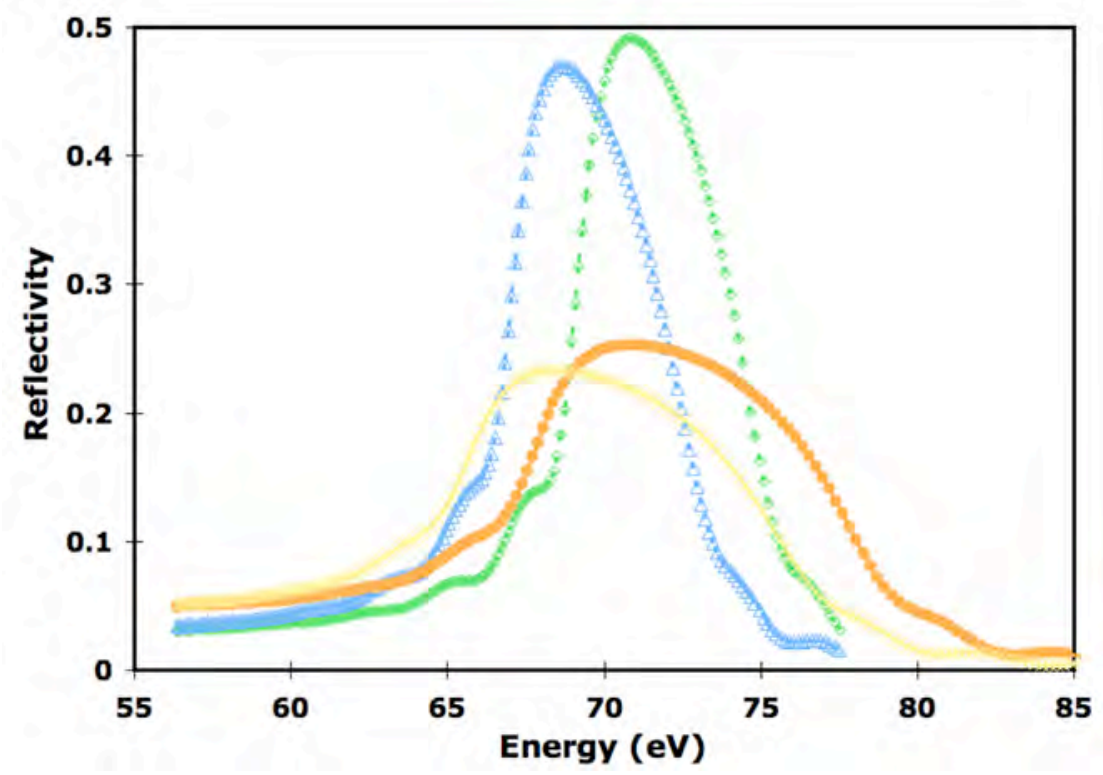

Figure 4.5: (a) Reflectivity as a function of energy at various grazing incidence angles for the $256 \mathrm{eV}$ spherical and turning mirrors. Open purple squares: spherical mirror at $83^{\circ}$ incidence angle; open blue triangles: spherical mirror at $85^{\circ}$ incidence angle; solid orange circles: turning mirror at $43^{\circ}$ incidence angle; yellow hatches: turning mirror at $45^{\circ}$ angle. (b) Reflectivity as a function of energy at various grazing incidence angles for the $68 \mathrm{eV}$ spherical and turning mirrors. Open green diamonds: spherical mirror at $75^{\circ}$ incidence angle; open blue triangles: spherical mirror at $85^{\circ}$ incidence angle; solid orange circles: turning mirror at $43^{\circ}$ incidence angle; yellow hatches: turning mirror at $45^{\circ}$ incidence angle. 
$\mathrm{eV}$ multilayers to alignment incidence angle is unsurprising due to the necessarily smaller bi-layer periods for the shorter wavelength.

Because for each imager the total mirror reflectivity is determined by the convolution of the reflectivity from both the spherical and the turning mirror, a slight mismatch of the central energy results in a considerable decrease in the two-mirror reflectivity. Thus, careful set-up alignment of the mirrors is critical for maximizing the signal collected.

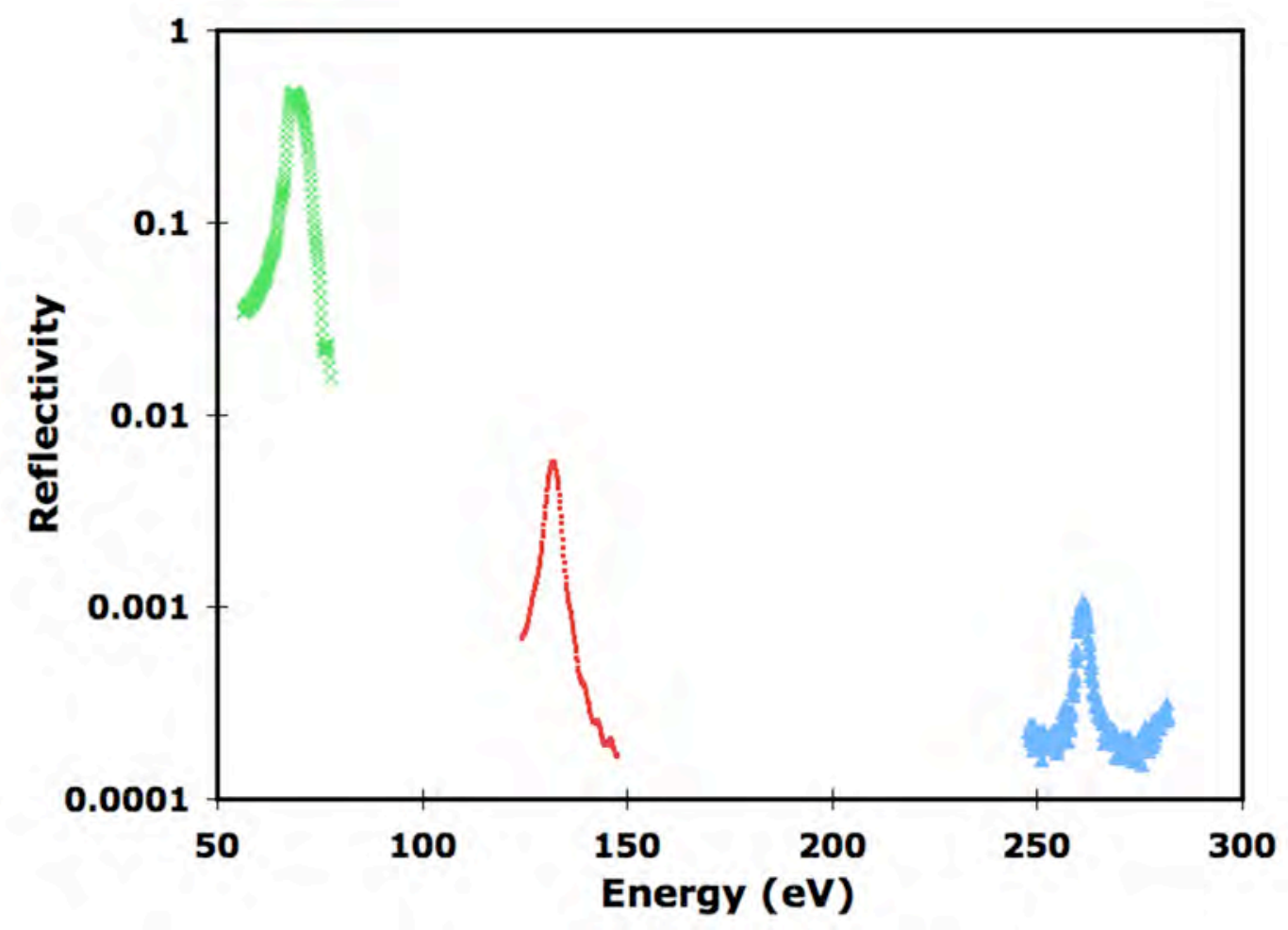

Figure 4.6: Comparison of reflectivity for different orders on the $68 \mathrm{eV}$ spherical mirror. All measurements were taken at $85^{\circ}$ grazing angle incidence. Green crosses: first order; red dotted line: second order; blue solid triangles: fourth order.

A calibration of the sensitivity of the $68 \mathrm{eV}$ spherical mirror for multiple orders of the imaging energy was also completed. Fig. 4.6 shows the reflectivity in first, second and fourth orders. It is clear that the reflectivity at higher orders is negligible. The reflectivity of second order $68 \mathrm{eV}$ light (which occurs slightly off the calculated second order, at $131 \mathrm{eV}$ instead of $136 \mathrm{eV}$ ) is down two orders of mag- 
nitude, from 0.46 to 0.005 . In fourth order, at $261 \mathrm{eV}$, the reflectivity is further decreased to 0.001 . The slight variation in the resonance energies of the higher orders is due to the refraction from vacuum to the first layer of the multilayer mirrors, which changes the effective incidence angle.

\subsubsection{Damage to Mirrors from Exploding Targets}

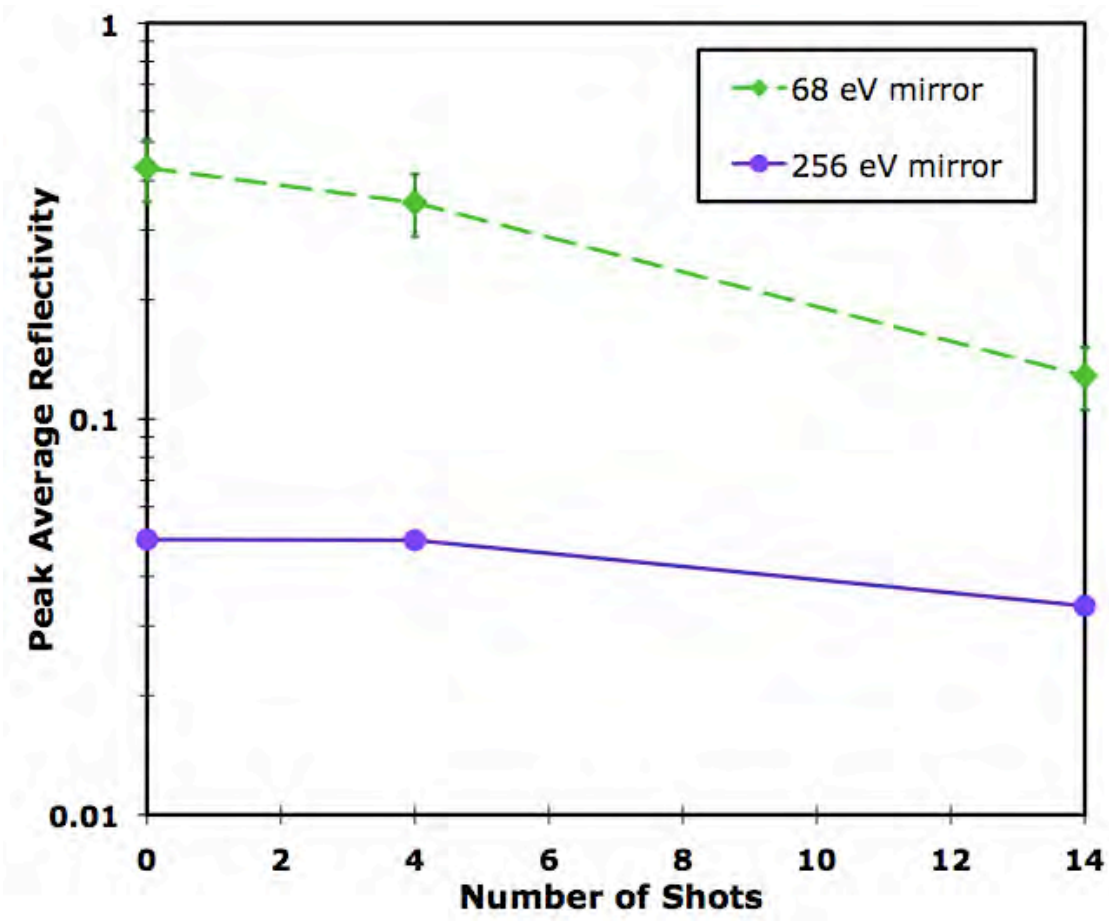

Figure 4.7: Effect of accumulation of debris on the mirror surface due to shot exposure, plotted as reflectivity as a function of number of shots. Green dotted line with diamonds is for the $68 \mathrm{eV}$ XUV spherical multilayer mirror and the purple solid line with circles is for the $256 \mathrm{eV}$ XUV spherical multilayer mirror. Error bars for the $256 \mathrm{eV}$ mirror are too small to be seen over the data marker itself.

Because nearly all materials are opaque to soft x-rays, it is impractical to shield the XUV mirrors from target debris while still maintaining their functionality, and so the mirrors are operated uncovered. Therefore, they are subjected to deposition of debris from the exploding target. This layer of small particles builds up on the exposed mirror face with subsequent laser shots, and can have a significant 
effect on the spherical mirrors' reflectivity, which is then manifested in a decreased brightness of the XUV image. Two spherical mirrors (one at each of the energies) were exposed to varying levels of deposition coating in different spots on the mirror, and were then calibrated to monitor the effect of successive laser shots on mirror reflectivity (the turning mirrors are placed farther down the imaging line and therefore not exposed to debris from the target). Fig. 4.7 shows the degradation of the spherical mirrors with increasing debris accumulation (quantified by the number of shots the mirror was exposed to). The reflectivity decreases by nearly $40 \%$ after a series of 14 shots on the same area of the $68 \mathrm{eV}$ mirror, whereas the $256 \mathrm{eV}$ spherical mirror reflectivity decreases by less than $2 \%$ over the same number of shots. Error bars are associated with the slight differences expected in debris deposition depending on the material and size of the target, as well as systematic errors in the calibration. In the case of the $68 \mathrm{eV}$ mirror, the error is estimated at $\sim 18 \%$, while the $256 \mathrm{eV}$ reflectivity error is $\sim 2 \%$ (in the plot, the error bars are not apparent over the size of the data marker). As $256 \mathrm{eV}$ light is more penetrating than $68 \mathrm{eV}$ light, it is therefore not as vulnerable to a thin layer of absorbing material. The typical operating procedure was to rotate the $2.5 \mathrm{~cm}$ diameter spherical mirror behind its $6 \mathrm{~mm}$ aperture to give a clean surface after a number of laser shots chosen to limit loss of reflectivity. Any multi-shot decrease of image brightness needs to be taken into account when calculating the absolute brightness of an XUV image.

\subsection{Experimental Results}

Experiments were performed on the Titan Laser at the Lawrence Livermore National Laboratory. The laser generates pulses of 500 to $700 \mathrm{fs}$ duration, and an energy of up to $150 \mathrm{~J}$, with $15 \%$ of that energy in a spot size of $5 \mu \mathrm{m}$, for a peak intensity on target of approximately $3 \times 10^{20} \mathrm{~W} / \mathrm{cm}^{2}$. Only three examples of targets are discussed here: $25 \mu \mathrm{m}$ thick copper foil, $10 \mu \mathrm{m}$ thick copper foil, and $10 \mu \mathrm{m}$ thick aluminum on the front side with $30 \mu \mathrm{m}$ thick copper on the back side. All targets are $1 \mathrm{~mm} \times 1 \mathrm{~mm}$ dimension. XUV emission from the rear side was 
(a)

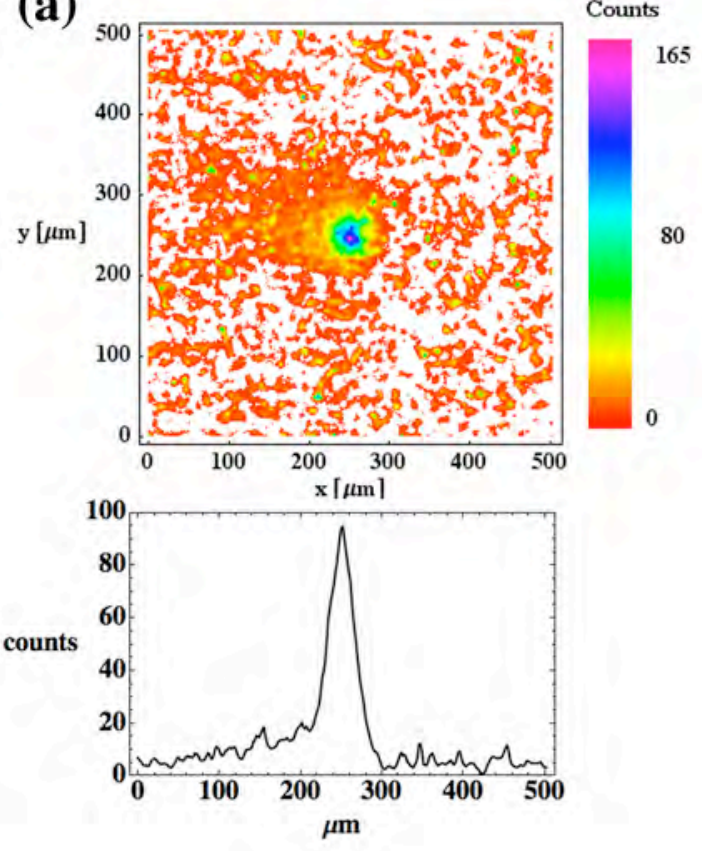

(b)
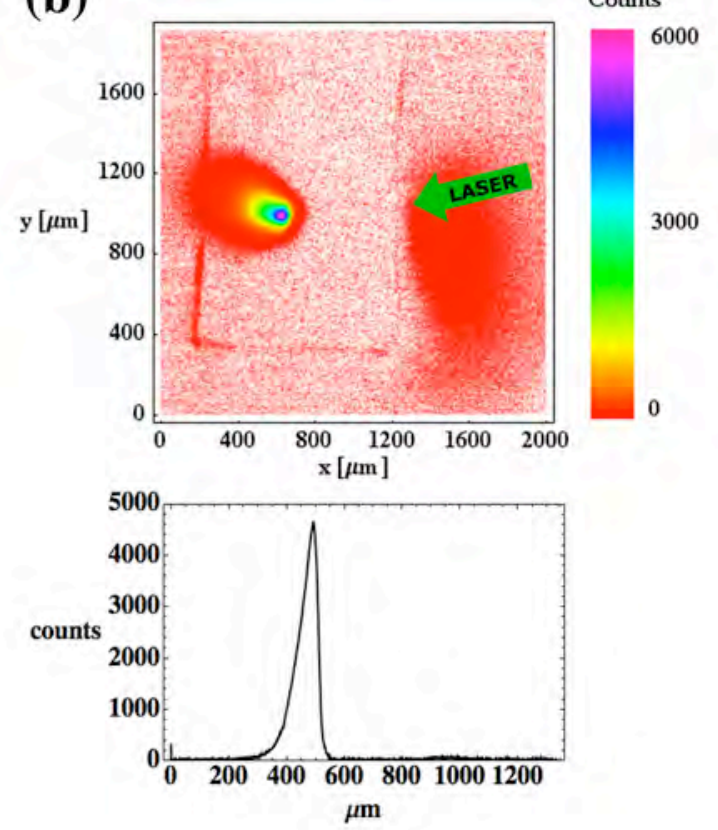

Figure 4.8: A $10 \mu \mathrm{m} \mathrm{Cu}$ target. (a) The raw $256 \mathrm{eV}$ image shown as CCD counts and lineout of the image; (b) the raw $68 \mathrm{eV}$ image shown as CCD counts and lineout of the $68 \mathrm{eV}$ image. The long time scale of bright emission at the $68 \mathrm{eV}$ energy allows the capture of the whole interaction region. 
imaged at a viewing angle of $34^{\circ}$ with respect to target normal. The experimental layout is shown in Fig. 4.2.

Fig. 4.8 shows the XUV images for a $10 \mu \mathrm{m}$ copper foil at $256 \mathrm{eV}$ (Fig. 4.8a) and $68 \mathrm{eV}$ (Fig. 4.8b), as examples of the raw data collected by the imagers, along with their counts profiles. Electron heating at the rear surface is visible at both 68 $\mathrm{eV}$ and $256 \mathrm{eV}$, although, as apparent from the line-outs, the emission at $68 \mathrm{eV}$ is at a much higher signal level, and displays a full width at half maximum (FWHM) that is more than two times as large. This is due both to the increased reflectivity of the $68 \mathrm{eV}$ mirrors over the $256 \mathrm{eV}$, as well as the much longer temporal and spatial scale of $68 \mathrm{eV}$ XUV emission. It is interesting to note that the $68 \mathrm{eV}$ image very obviously displays the edges of the target, the front-side plume which can be seen around the side of the target, and the plasma plume at the back, which extends beyond the edge of the target. This shows that the $68 \mathrm{eV}$ XUV imager observes not just surface characteristics of heating, but the expansion of the lower density hot plasma as well. There is negligible evidence for plume development in the $256 \mathrm{eV}$ images.

By applying the calibration results discussed above for the integral reflectivities of the multilayer mirrors, while also taking into account the collection solid angle, image magnification, CCD camera settings, detector quantum efficiency, CCD pixel size, mirror bandwidths and filter transmission, an absolute time integrated XUV emission can be derived for each pixel of the CCD camera image. This image intensity is proportional to emission from the target in a bandwidth $/ \mathrm{cm}^{2} /$ steradian. The typical target peak intensities obtained for the images shown are $1.44 \mathrm{~J} / \mathrm{cm}^{2} / \mathrm{sr}$ in a $10 \%$ bandwidth and $19.14 \mathrm{~J} / \mathrm{cm}^{2} / \mathrm{sr}$ in a $10 \%$ bandwidth for $68 \mathrm{eV}$ and 256 $\mathrm{eV}$ imagers, respectively. (A $10 \%$ bandwidth refers to photons in the range corresponding to $10 \%$ of the peak energy, i.e. $6.8 \mathrm{eV}$ around the $68 \mathrm{eV}$ peak, and $25.6 \mathrm{eV}$ for the $256 \mathrm{eV}$ peak.) The absolute intensities obtained in this manner are compared with theoretical intensities obtained from LASNEX calculations as shown below. 


\subsection{LASNEX Calculations}

The radiative-hydrodynamics code LASNEX[81] was used for model calculations in 2-D, assuming a thin foil with an initial Gaussian radial temperature pattern having $100 \mu \mathrm{m}$ FWHM and uniform temperature as a function of depth in the foil. It was assumed that the plasma expands with cylindrical symmetry from the rear surface of the target, and thus 2-D LASNEX is sufficient to model the full expansion of the plasma.

A series of LASNEX simulations were run with a different initial peak temperature. At each point in the plasma, LASNEX calculated the density, electron temperatures and emissivity. An integration of emissivity over time and within a narrow band of photon energy was calculated from the hot plume edge into the plasma up until the opaque surface, along the line-of-sight to the spot of maximum target heating (i.e., target center). Because the experimental view angle was $34^{\circ}$ to the target normal, emission in the synthetic images was integrated at that same view angle.

A series of LASNEX simulations was also run for each target type with the different initial target temperatures, and in each case, an absolute XUV brightness for the hot spot was determined for the specific view angle. By plotting the set of LASNEX model results of XUV brightnesses expected for each target temperature, Fig. 4.9 was composed for different thicknesses of copper slabs. The output is emission in absolute units of $\mathrm{J} / \mathrm{cm}^{2} /$ steradian as a function of initial temperature.

As evidenced in Fig. 4.9, LASNEX predicts that for a given XUV imaging energy and single viewing angle, the temperature-absolute brightness curve is very similar for slabs of the same material but different thicknesses. These thin targets expand quickly from the surface and the specific brightness seen in the XUV range falls rapidly away from the plasma.

For each target shot, the experimental XUV image was processed into absolute brightness, and then converted to temperature maps by point-wise mapping using comparison with the synthetic LASNEX simulations. Figures 4.10(a) and 4.10(b) show the electron temperature maps for a $10 \mu \mathrm{m}$ copper foil based on each channel of the XUV. 


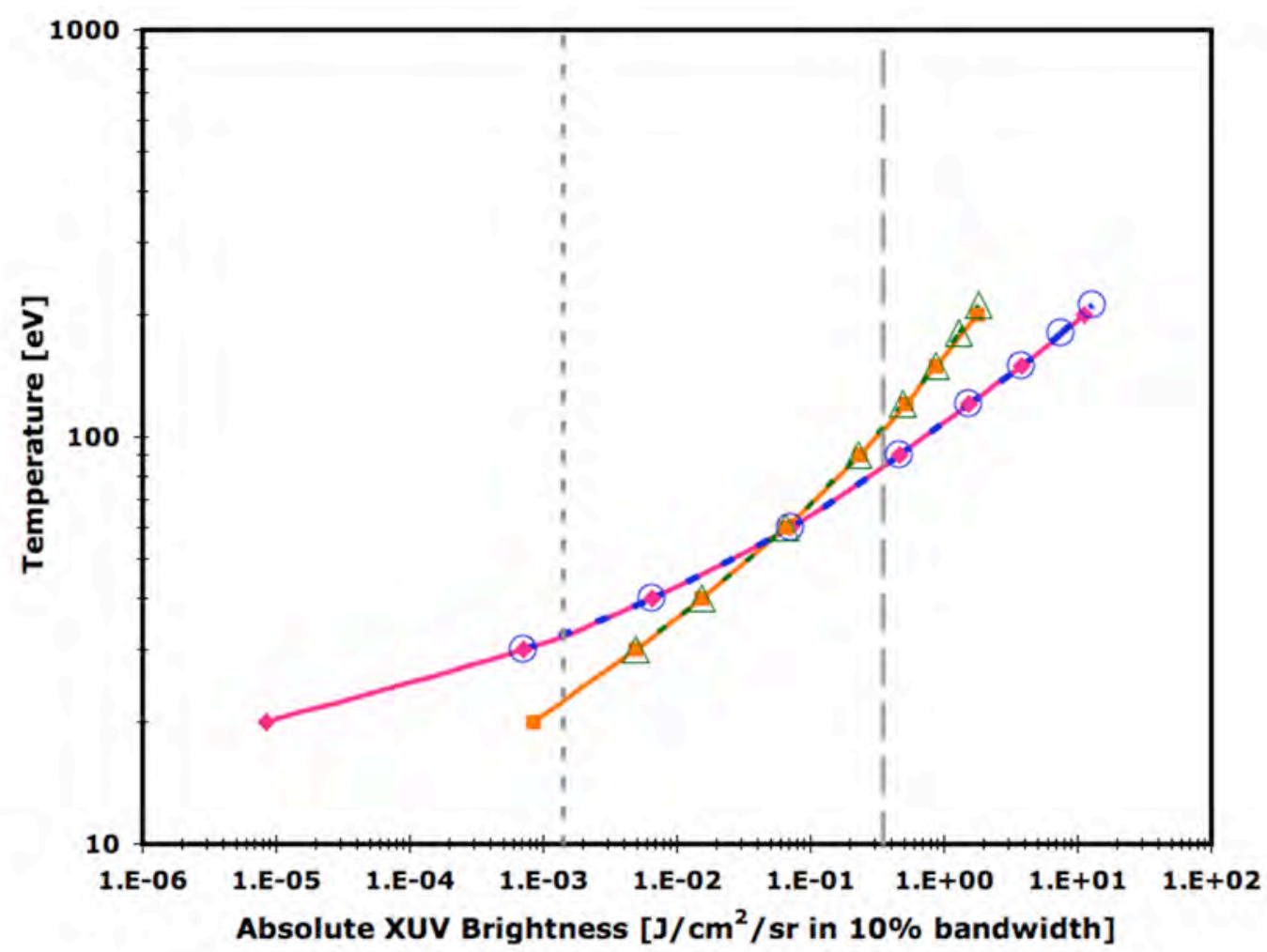

Figure 4.9: LASNEX simulation results: peak target pre-expansion temperature vs absolute brightness for a selection of flat foil targets. Solid pink diamonds: $25 \mu \mathrm{m}$ $\mathrm{Cu}$ at $256 \mathrm{eV}$; solid orange squares: $25 \mu \mathrm{m} \mathrm{Cu}$ at $68 \mathrm{eV}$; open blue circles: $10 \mu \mathrm{m}$ $\mathrm{Cu}$ at $256 \mathrm{eV}$; open green triangles: $10 \mu \mathrm{m} \mathrm{Cu}$ at $68 \mathrm{eV}$; large dashed vertical line: $256 \mathrm{eV}$ detection threshold; small dashed vertical line: $68 \mathrm{eV}$ detection threshold.

Because the point-wise mapping for the entire target was completed using only the single integral of emissivity derived from the hot spot, the temperature maps of Fig. 4.10 must be interpreted with the caveat that only the temperature of the hot spot region is a valid determination. The temperatures assigned to the obliquely viewed expanded plume with lines of sight that do not connect to the heated region of the rear surface are not meaningful.

It is for this reason that the $256 \mathrm{eV}$ XUV imager produces a more relevant temperature map because emission at the $256 \mathrm{eV}$ photon energy is so short-lived that the majority of it occurs before significant expansion from the rear surface. Thus, the only region that appears to emit is the heated spot itself, and the pointto-point mapping from absolute brightness to temperature is nearly fully valid. 

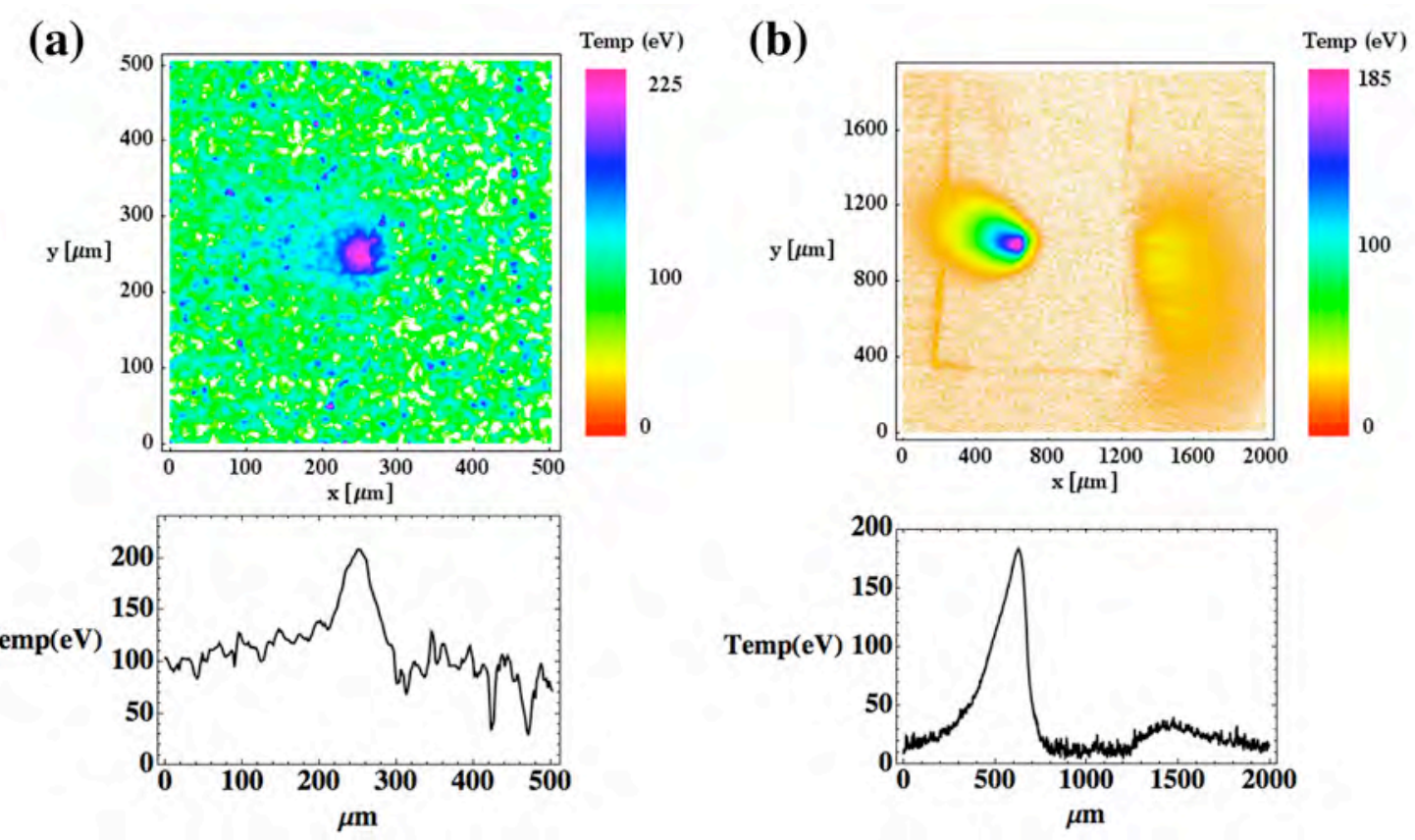

Figure 4.10: (a) Temperature map and lineout for the $10 \mu \mathrm{m} \mathrm{Cu}$ target based on the $256 \mathrm{eV}$ XUV imager. (b) Temperature map and lineout for the $10 \mu \mathrm{m} \mathrm{Cu}$ target based on the $68 \mathrm{eV}$ XUV imager. 
This method accordingly provides an adequate means of establishing peak temperature of the back surface, and from Fig. 4.10, the $68 \mathrm{eV}$ XUV imager displayed a peak temperature of $187 \mathrm{eV}$, while the $256 \mathrm{eV}$ XUV imager showed a peak temperature of $225 \mathrm{eV}$.

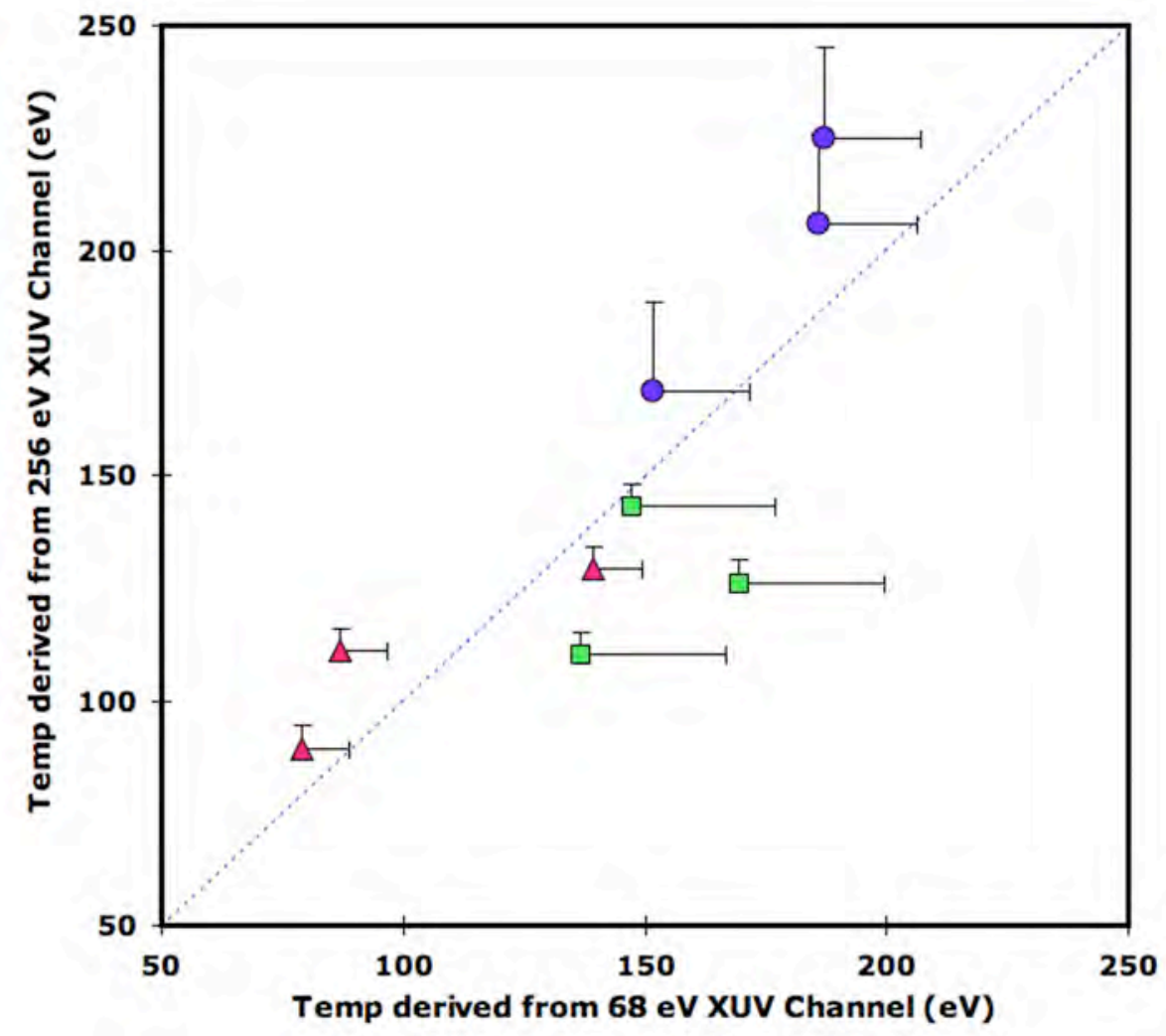

Figure 4.11: Peak temperatures derived from the two different XUV imagers plotted against each other. Squares: $25 \mu \mathrm{m} \mathrm{Cu}$; circles: $10 \mu \mathrm{m} \mathrm{Cu}$; triangles: $10 \mu \mathrm{m}$ $\mathrm{Al} / 30 \mu \mathrm{m} \mathrm{Cu}$. The diagonal line indicates equivalent temperatures from both imagers.

Peak temperatures for the other target types described were also determined. For each case, the temperatures from the $68 \mathrm{eV}$ channel were plotted against those from the $256 \mathrm{eV}$ channel in Fig. 4.11. Error bars account for uncertainties due to the debris deposition on the spherical mirrors. In each case, the temperatures matched up to within $40 \mathrm{eV}$. Target rear surface temperatures varied between 75 and $225 \mathrm{eV}$, with thinner targets demonstrating higher levels of heating (see Fig. 4.12). The higher level of heating in thinner targets is due to both the lower 
mass per unit area and the refluxing of electrons (multiple passes of the electrons through the target) as discussed in detail in Ref. [82].

The analytic fitting to the LASNEX model data used to obtain the temperature maps deviates by no more than $3 \%$ from the LASNEX data. The Poisson error on the number of photons absorbed per pixel is $7 \%$ in the case of the $256 \mathrm{eV}$ imager, and $1 \%$ for the $68 \mathrm{eV}$ imager. The resulting temperature Poisson error is $2 \%$ and $0.5 \%$ for the $256 \mathrm{eV}$ and the $68 \mathrm{eV}$ imagers, respectively. The systematic uncertainties in the peak temperature data due to difference between 1-D modeling and the real situation addressed in the $\mathrm{Gu}$, et al. paper are not a factor in this analysis because the 2-D LASNEX simulations in this case can sufficiently accurately model the plasma plume expansion.

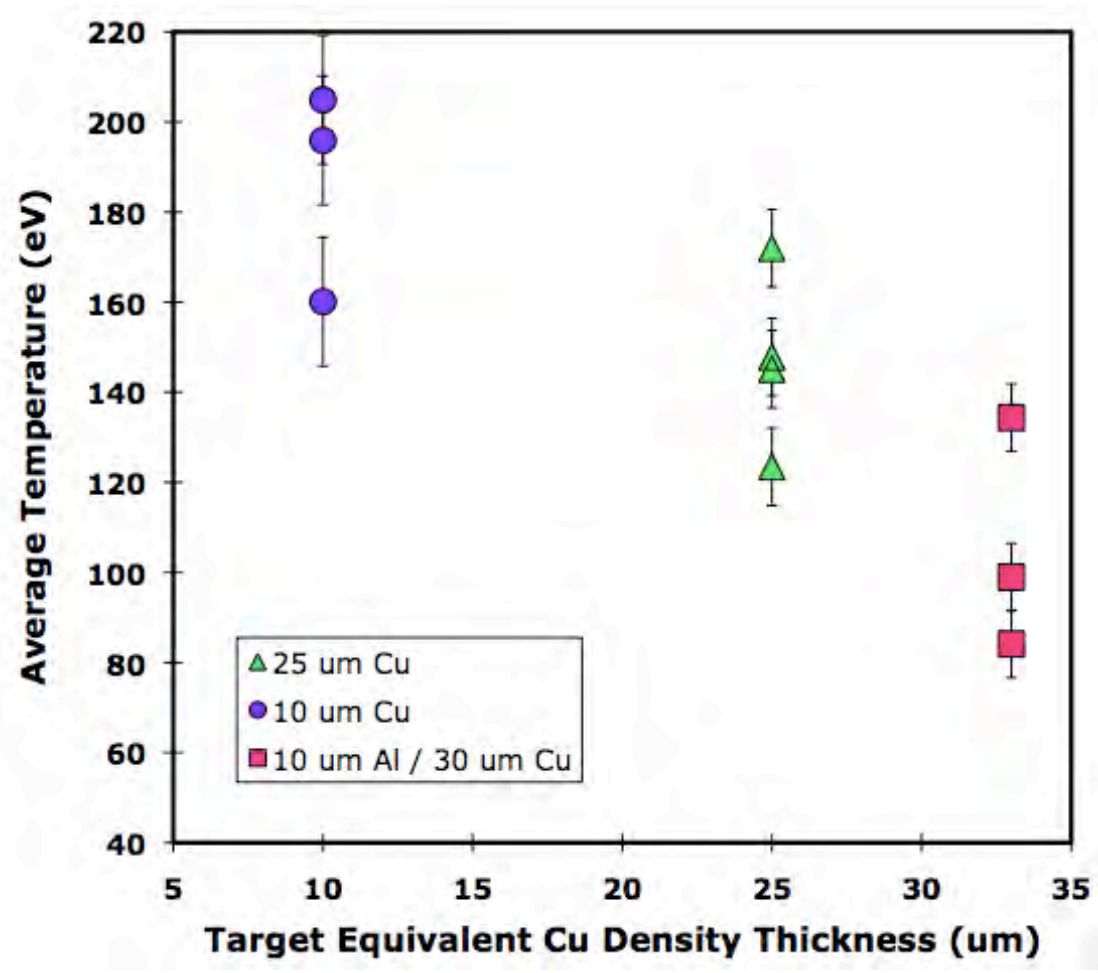

Figure 4.12: Target backside peak temperatures as a function of target equivalent $\mathrm{Cu}$ density thickness. Triangles: $25 \mu \mathrm{m} \mathrm{Cu}$; circles: $10 \mu \mathrm{m} \mathrm{Cu}$; squares: $10 \mu \mathrm{m} \mathrm{Al}$ / $30 \mu \mathrm{m} \mathrm{Cu}$. 


\subsection{Discussion: The Use of the XUV Imagers}

It has been shown here that XUV imaging at 68 and $256 \mathrm{eV}$ gives a reliable measurement of temperature in a range useful for studies of heating of thin foil targets by petawatt class lasers. In the temperature range between $75 \mathrm{eV}$ and 225 $\mathrm{eV}$, XUV diagnostic has been validated against itself by providing evidence that the two imagers give consistent temperatures to within $40 \mathrm{eV}$. Each imager has a temperature range in which it is useful bounded by a sharp fall in signal at the low temperature limit and by increasing afterglow and imaging of the expansion plume at the high temperature limit. The experimental detection thresholds are marked on Fig. 4.9, where for the $256 \mathrm{eV}$ channel, it is at $0.35 \mathrm{~J} / \mathrm{cm}^{2} / \mathrm{sr}$ in a $10 \%$ bandwidth (or, $89 \mathrm{eV}$ for thin $\mathrm{Cu}$ foils), and for $68 \mathrm{eV}$, it is at $0.0014 \mathrm{~J} / \mathrm{cm}^{2} / \mathrm{sr}$ in $10 \%$ bandwidth (or, $16 \mathrm{eV}$ for thin $\mathrm{Cu}$ foils).

As demonstrated by the LASNEX simulations (of Fig. 4.9), both XUV imagers are better predictors for lower temperatures because the change in signal (absolute brightness) with temperature is very rapid - a large change in absolute brightness only corresponds to a small change in temperature. However, at higher temperatures, the Planckian radiation in the narrow photon energy band imaged begins scaling linearly with temperature when $h \nu / k T$ becomes small. Thus, at higher temperatures, calibration uncertainties come into play when attempting to accurately derive a temperature from the absolute XUV brightness. This limitation plus the longer afterglow and associated imaging of the expansion plume (which then yield more uncertainty in the modeling as temporal and spatial integration increase, as well as saturation limits in the diagnostic) limit the maximum feasible temperature measurement to about $2-3 \times$ the photon energy, as evidenced by measurements up to $190 \mathrm{eV}$ for the $68 \mathrm{eV}$ channel.

Tests were conducted in which a fused silica plate was placed in the imaging line to block the XUV light, but allow residual light of other forms (i.e., OTR, IR, UV, etc.) to be transmitted. It was found that at a temperature less than approximately $100 \mathrm{eV}$, these other forms of light become non-negligible in comparison to XUV light in the $256 \mathrm{eV}$ channel, raising the lower limit slightly above the detection threshold temperature of $89 \mathrm{eV}$ quoted previously. 
In effect, the useful application of the techniques described here are limited to an operational temperature threshold of $16 \mathrm{eV}$ using the $68 \mathrm{eV}$ photon energy imager, and a temperature of $100 \mathrm{eV}$ using the $256 \mathrm{eV}$ photon energy imager, and an upper temperature limit of approximately $2-3 \times$ the imaging photon energy for each respective imager. Thus, the $256 \mathrm{eV}$ imager is a logical choice for imaging of higher temperatures, while the $68 \mathrm{eV}$ imager is more effective for slightly lower energies.

\subsection{Spectroscopy in the XUV}

\subsubsection{The XUV Flat-Field Spectrometer}

A flat-field grazing-incidence spectrometer[63] was also employed over a similar XUV energy range $(50-400 \mathrm{eV}, 30-250 \stackrel{\circ}{A})$ to provide one-dimensional spatially resolved spectroscopic measurements. By analyzing the spectra, the temperatures derived from the two XUV images could also be independently verified.

The Flat-Field Spectrometer (FFS) uses a concave 1200 lines/mm aperiodic grating set at a glancing angle of $4.6^{\circ}$. As explained in Chapter 3, the use of a concave aperiodic grating allows the spectrum to be projected onto a flat plane, rather than onto the Rowland circle (which requires a curved imaging surface), as in the case of using a standard concave grating. In the case described here, the flat detector was a vacuum charge coupled device (CCD) with a back-thinned $1340 \times 1300$ array with $20 \mu \mathrm{m}$ pixel size. To increase the flux density at the detector plane, a cylindrical Au mirror is set at a glancing angle of $4^{\circ}$, with its surface orthogonal to the flat-field grating. A limited aperture filter between the grating and detector discriminates against fluorescent and scattered emission within the spectrometer. See Fig. 4.13 for a schematic of the FFS diagnostic.

The diffraction grating is located at a distance of $1.25 \mathrm{~m}$ from the target being imaged, and the detector plane is $0.235 \mathrm{~m}$ from the grating center. The view angle is $34^{\circ}$ with respect to target rear side normal The solid angle subtended by the spectrometer is $5.69 \times 10^{-5} \mathrm{sr}$ and spatial magnification is 0.6 (demagnification) giving a resolution of approximately $35 \mu \mathrm{m}$. 


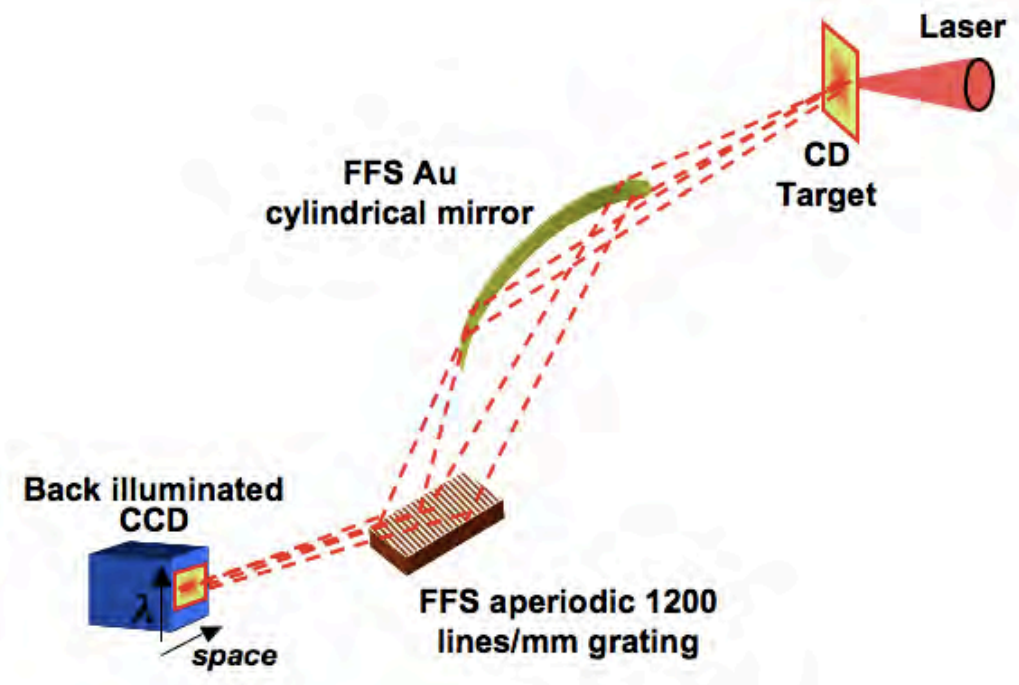

Figure 4.13: Schematic of flat field spectrometer setup.

To align the spectrometer, the grating and mirror were first positioned at the required distance. The angles of the grating and mirror are adjusted by sending a HeNe laser in, and the resulting spot is imaged onto the center of the CCD chip (where both the angle of the grating and the location of the CCD are defined by the wavelength range we wish to image over). Using a point source at target chamber center (an optical fiber illuminated by a HeNe works quite well), the curve of the $\mathrm{Au}$ mirror is then adjusted to focus the image at the CCD plane. Screens are also arranged to eliminate light not passing the mirrors and which may be scattering up into the detector or which may be of a higher order.

\subsubsection{Spectroscopy Results}

The use of the cylindrical mirror in conjunction with the grating in the FFS allows for both spatial imaging and spectral imaging/dispersion. Indeed, for most targets, a double spectrum one originating from the target rear plume, and one from the front plasma plume is seen in the flat-field data. Fig. 4.14 shows spectra recorded on a single shot from both the front and rear side of the target. (recorded with no low-pass filter). The spectrum shows a rear surface Planckian continuum 
with super-imposed line radiation. The bright continuum is emitted from the high density, hot, optically thick rear surface of the solid target. As the lower density plasma plume forms on the backside and moves away from the surface, spectral lines become visible. The different fall-off rates for the individual spectral lines is evidence for a plume changing in density and temperature as it expands from the surface. The spectra associated with the front surface plasma plume does not display any bright continuum, indicating that the plume visible on the front side from around the edge of the target is already of a low enough density to be optically thin to spectral lines in the XUV region.

Line radiation from an integrated region of roughly $300-600 \mu \mathrm{m}$ from the back surface, and 200 - $500 \mu \mathrm{m}$ from the front surface of a $25 \mu \mathrm{m}$ CD planar target are identified. Populations of the CVI (carbon hydrogen-like) and CV (carbon helium-like) ionic stages and their relative intensities are determined.

The atomic spectroscopy code FLYCHK[83] is used to calculate line profiles of atomic transitions. FLYCHK uses a detailed level structure for lithium-like, helium-like and hydrogenic ion stages to calculate charge state and population distributions. Spectra for densities between $\mathrm{n}_{e}=1 \times 10^{18}-1 \times 10^{21} \mathrm{~cm}^{-3}$ and temperatures from $10-1000 \mathrm{eV}$ are individually generated for both steady-state local thermodynamic equilibrium (LTE) and non-LTE conditions. In both cases, solutions are assumed to allow a single temperature and pressure to be attributed to the whole system for the sake of simplifying the problem. Line pair ratios of intensities of selected ionization transitions are determined from the synthetic spectra and then compared against the experimental spectra. It is found that at a given temperature, ratios do not significantly change for the different plasma densities. In both the LTE and non-LTE cases, it is assumed that the lines used for temperature measurements are not subject to reabsorption or induced emission.

The rear surface emission spectrum from a $25 \mu \mathrm{m}$ CD target irradiated at 144 $\mathrm{J}, 0.5 \mathrm{ps}$, with a $50 \mu \mathrm{m}$ focal spot is analyzed. From the FFS, the experimental ratios of hydrogenic to helium-like lines integrated over the region $300-600 \mu \mathrm{m}$ from the back surface is determined: CVI 4p-2s (Balmer beta, $\lambda=135 \AA$ ) to CV 4 -2s (helium-like Balmer beta analogue, $\lambda=173 \AA$ ) is 0.3 ; CVI 3p-2s (Balmer 


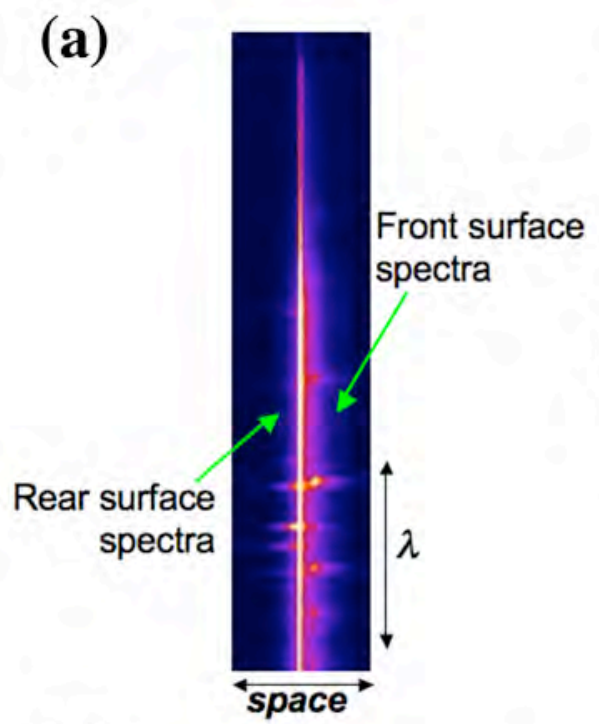

\section{(b)}

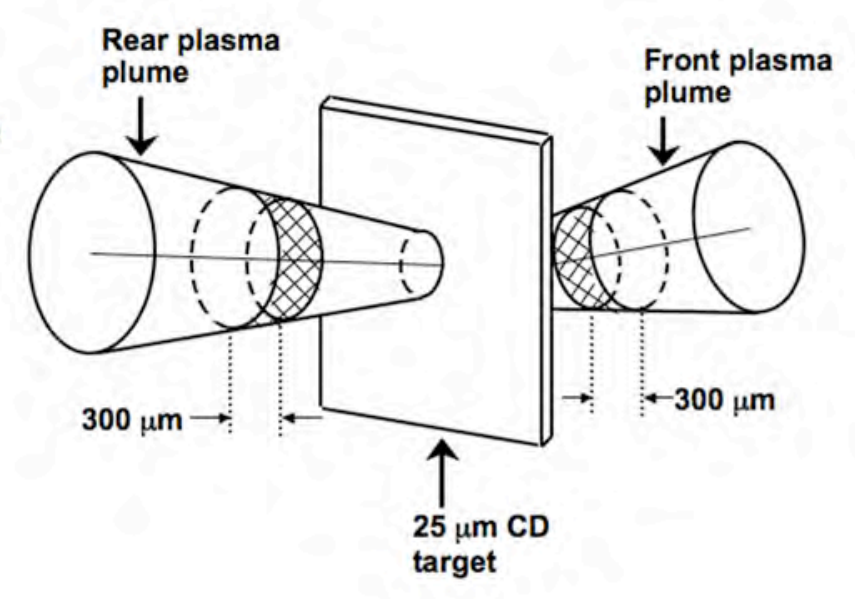

(c)

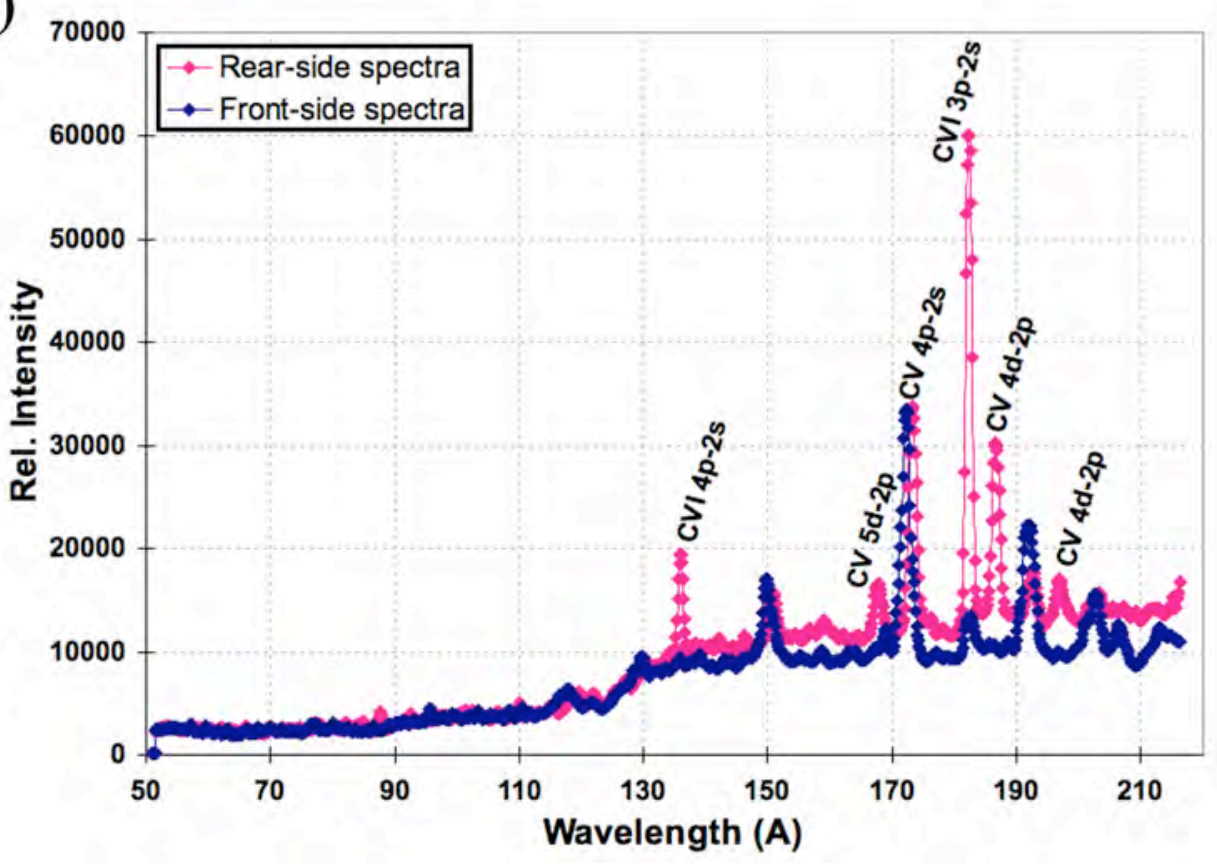

Figure 4.14: (a) The soft x-ray spectra for a CD planar target using a 1200 lines/mm diffraction grating. (b) Schematic illustration of the spatial zone from which plasma emission was recorded. (c) The lineouts for the front and rear surface spectra.

alpha, $\lambda=182 \AA$ ) to $\mathrm{CV} 4 \mathrm{~d}-2 \mathrm{p}$ (helium-like Balmer beta analogue, $\lambda=187 \AA$ ) is 2.5; and CVI 3p-2s to CV 5d-2p (helium-like Balmer gamma analogue, $\lambda=167$ $\AA$ ) is 7.1. After comparison to synthetic spectra for plasma density $\mathrm{n}_{e}=1 \times 10^{18}$ 
$\mathrm{cm}^{-3}$ (the density at which the experimental spectrum most closely matches the synthetic spectrum shape), this predicts a plasma temperature of $67.6 \mathrm{eV}, 75.9$ $\mathrm{eV}$, and $79.3 \mathrm{eV}$ from each of the line pair ratios, respectively in the LTE case, and plasma temperatures of $64.8 \mathrm{eV}, 78.5 \mathrm{eV}$, and $80.2 \mathrm{eV}$ in the non-LTE limit. Fig. 4.15 compares the predicted line ratios with the experimental results. The average temperature of the rear plasma plume 300 - $600 \mu \mathrm{m}$ from the back surface is thus $74.4 \mathrm{eV} \pm 0.1 \mathrm{eV}$, assuming minimal opacity effects.

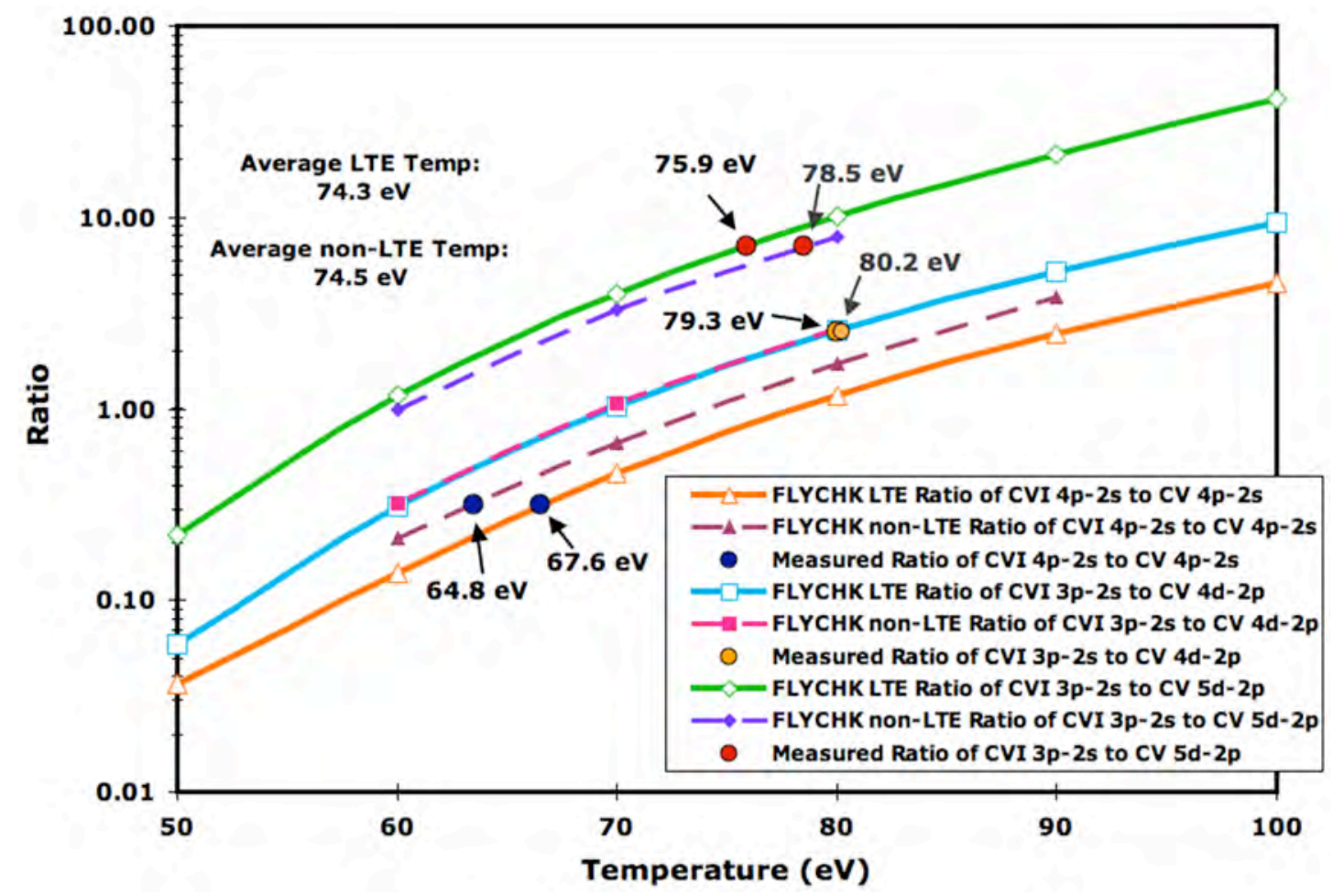

Figure 4.15: The comparison of measured experimental line ratios for spatial zone marked in Fig. 4.14 with expected line pair ratios generated using the FLYCHK spectroscopic code.

Analysis of the 2-D spatially resolved $68 \mathrm{eV}$ XUV and $256 \mathrm{eV}$ XUV images show pre-expansion peak temperatures of the rear surface of $127 \mathrm{eV}$ and $154 \mathrm{eV}$, respectively for the same $25 \mu \mathrm{m}$ CD target. The temperatures calculated from the two XUV imagers, while fairly consistent with each other, are approximately $70 \mathrm{eV}$ higher than the temperature predicted by the FFS (nearly a factor of 2). The XUV imager measurement is derived from the optically thick continuum of 
the dense hot phase of the plasma, whereas the flat-field spectrum is taken from the low density plasma plume; thus, the lower temperature obtained represents an average over the cooling phase of the plasma.

If a pure adiabatic cooling of the plasma is assumed as it expands from the target rear surface, then based on a change in temperature of a factor of 2 , it would be expected that the plasma would expand by nearly $3 \times$. However, the spatial scaling of the flat-field spectra shows that the plasma expands far more, and thus there is evidence of frozen ionization that leads to an overestimation of the plume temperature.

The feasibility of fast ignition depends on the capability of fast electrons to deposit their energy and heat the background material. An accurate measurement of the thermal emission from laser-irradiated targets can provide useful information about the properties of the matter, as well as the electron distribution driving the heating. Here we have demonstrated the usefulness of the 68 and $256 \mathrm{eV}$ XUV imaging diagnostics to provide 2-D spatially resolved temperature maps. In addition, a comparison has been made of plasma temperature at the rear surface and in the rear plasma plume of a planar target by additionally using an XUV spectrometer. These techniques reveal information about the 3-D plasma temperature and expansion characteristics.

Chapter 4 is a reprint of the material as it appears in T. Ma, Review of Scientific Instruments 79, 093507 (2008); T. Ma, Review of Scientific Instruments 79, 10E312 (2008); and T. Ma, IEEE Transactions on Plasma Science, Vol. 36, No. 4 (2008). The dissertation author was the primary investigator and author of these papers. 


\section{Chapter 5}

\section{Transport of Energy by \\ Ultra-Intense Laser-Generated \\ Electrons in Nail-Wire Targets}

\section{$5.1 \quad$ Introduction}

Among the complex physics parameters that govern the viability of cone-guided FI is the efficiency with which laser light can be converted to fast electrons, and how well the electrons can be transported over a path length of $\sim 100 \mu \mathrm{m}[84]$. Within this chapter, an experiment is described to characterize the physics of laser energy deposition and fast electron transport using a novel nail-wire target.

Cone-wire targets, in which a thin wire was joined to the cone tip, were previously irradiated to investigate aspects of hot electron flow in a solid beyond the cone without the complexities of a diverging beam[14, 85, 86, 87]. However, it was found that the physics of the laser interaction within the cone was often difficult to diagnose, and the use of a cone interface with a wire adds additional complexity in the glue joint between the wire and the cone.

In an attempt to avoid these complications, the simple low mass nail target consisting of a $1 \mathrm{~mm}$ long, $20 \mu \mathrm{m}$ diameter copper wire drawn directly from an $80 \mu \mathrm{m}$ hemispherical head was chosen for investigation of electron transport. It 
was found that Ohmic stopping played a dominant role in the propagation of hot electrons down the wire, and a hot surface layer in the wire could be attributed to a surface current of fast electrons.

The chapter is organized as follows: the experimental setup and diagnostics are described in Section 5.2. In Section 5.3, experimental results and analysis are presented. Section 5.4 presents ePLAS simulation results. Section 5.5 is a discussion and comparison of the experiment and modeling.

\subsection{Experimental Setup}

The experiment was conducted at the Vulcan Petawatt Laser at the Rutherford Appleton Laboratory. The Nd:Glass $1053 \mathrm{~nm}$ laser generated a pulse of 1 ps duration with an on-target energy of $309 \mathrm{~J}$. An f/3 off-axis parabola focused approximately $30 \%$ of the total laser energy into a $7 \mu \mathrm{m}$ FWHM focal spot with the remaining energy in a broader distribution around the central spot, giving a peak laser intensity of $2 \times 10^{20} \mathrm{~W} / \mathrm{cm}^{2}$. The target geometry and diagnostic configurations are shown in Fig. 5.1.

Three different diagnostics were used to measure the copper K $\alpha$ emission from the target. $\mathrm{Cu} \mathrm{K} \alpha$ x-ray radiation at $8048 \mathrm{eV}$ is predominantly due to binary collisions of $\mathrm{Cu}$ atoms with the hot electrons, and so its intensity is closely related to the rate of collisional heating. The number of recorded $\mathrm{K} \alpha$ photons is assumed proportional to the number of fast electrons[88].

$\mathrm{A} \mathrm{Cu} \mathrm{K \alpha}$ Bragg reflection crystal imager[65] gave two-dimensional time-integrated spatial information by detecting $\mathrm{x}$-ray radiation within a narrow $(\sim 6 \mathrm{eV})$ bandwidth around $8048 \mathrm{eV}$. The imager consisted of a $\mathrm{SiO}_{2} 211$ spherical quartz crystal with a radius of curvature of $38 \mathrm{~cm}$, placed $20 \mathrm{~cm}$ from the target. The crystal operated $1.3^{\circ}$ off axis in second diffraction order and with $15 \times$ magnification imaging onto a Fujifilm BAS-SR image plate. Resolution of the imager was approximately $20 \mu \mathrm{m}$. The view angle was in the horizontal plane at $29.4^{\circ}$ from the nail-wire axis. While the narrow bandwidth of this diagnostic allowed for monochromatic imaging, this was also one of its limitations: The K $\alpha$ spectral line shifts and broadens 


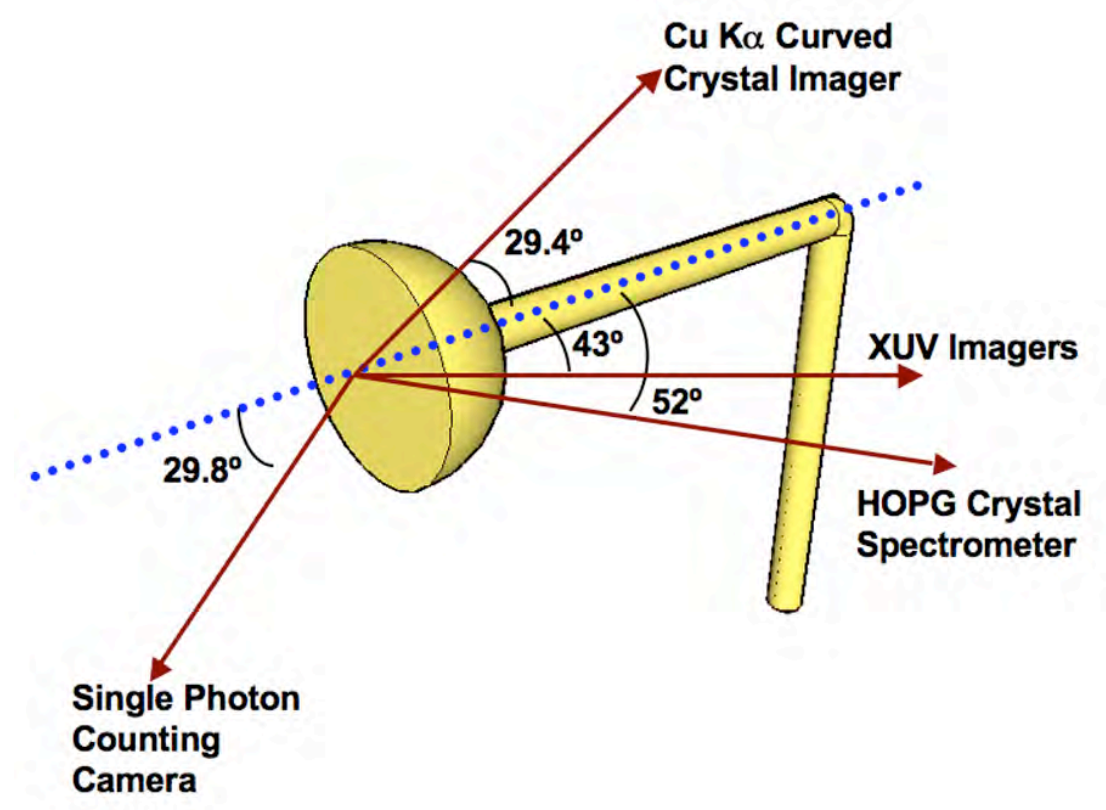

Figure 5.1: Layout of experimental setup showing diagnostic view angles relative to the nail-wire target axis.

with increasing target temperature, and so, for sufficient temperature increases, shifts off the narrow bandwidth of the crystal[66]. This effect was discussed in detail in Section 3.6.3.2. The collection efficiency of the $\mathrm{Cu} \mathrm{K} \alpha$ imager was thus dependent on the temperature of the target, and corrections were made for the loss in signal due to it.

In order to make an absolute measurement, two other $\mathrm{Cu} \mathrm{K} \alpha$ diagnostics were used for cross-calibration. The HOPG (highly oriented pyrolitic graphite) Bragg crystal spectrometer[67] provided a spectrum of the x-ray radiation. The reflected x-rays were measured over a $3.5 \mathrm{keV}$ spectral bandwidth (centered about 8048 $\mathrm{eV}$ ), with a spectral resolution of $5 \mathrm{eV}$, at a $52^{\circ}$ view angle on the rear-side of the nail-wire axis. The crystal was located $25 \mathrm{~cm}$ from both the x-ray source and the imaging plane. The spectrum was also recorded on Fujifilm BAS-SR image plates.

The single photon counting camera[71] had a CCD (charge-coupled device) camera configured such that no more than one photon hit each CCD pixel, so that the CCD counts were proportional to photon energy and a histogram of the data 
gave a measure of K-shell radiation emission that was absolutely calibrated. A Spectral Instruments Series 800 CCD with a pixel size of $13.5 \mu \mathrm{m}$, and a 2048 x 2048 pixel array recorded spectra through a $150 \mu \mathrm{m} \mathrm{Cu}$ filter. The CCD was positioned $380 \mathrm{~cm}$ from TCC, with a front view of $29.8^{\circ}$ to the nail-wire axis.

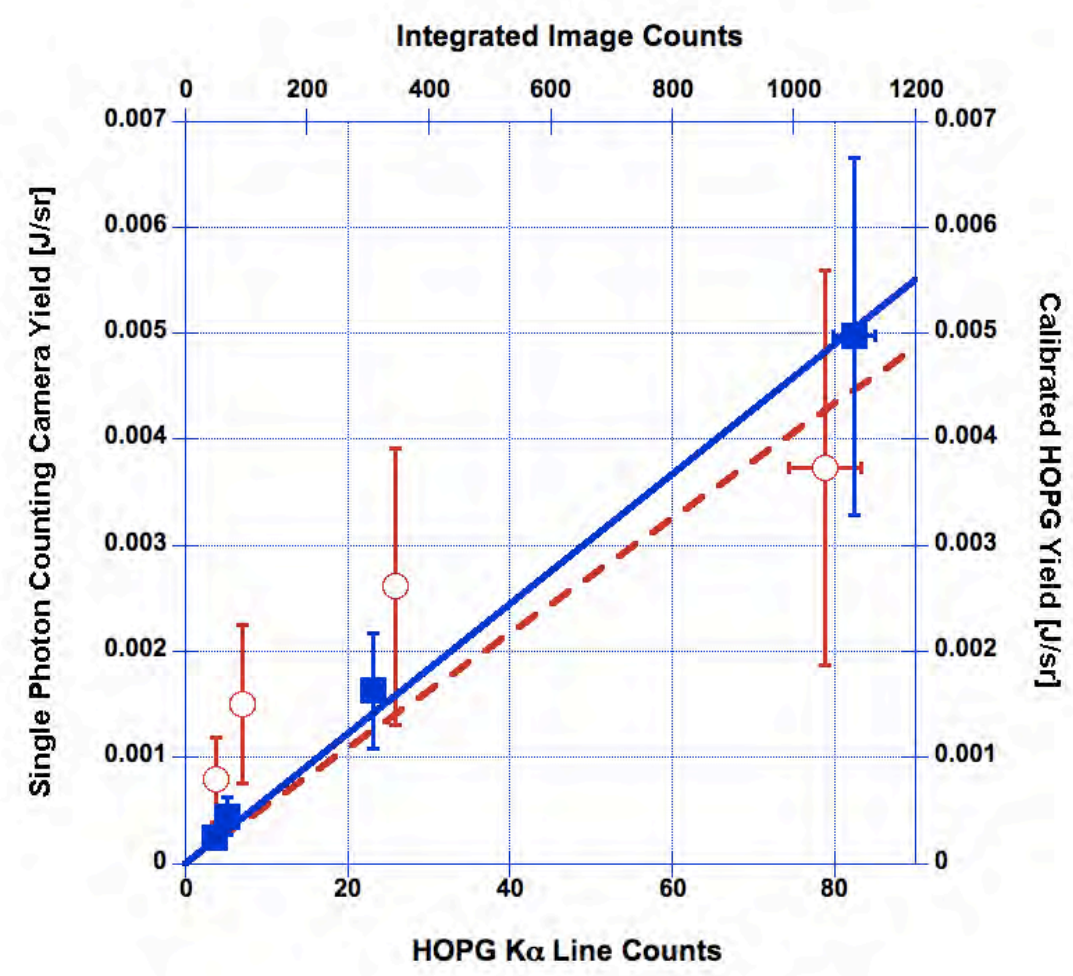

Figure 5.2: Cross-calibration of Single Photon Counting Camera K $\alpha$ yield vs. HOPG yield to determine absolute number of $\mathrm{Cu} \mathrm{K} \alpha$ values from $\mathrm{Cu} \mathrm{K} \alpha$ 2-D images. Dotted line is the least squares fit to the open circles (denoting Single Photon Counting Camera yields plotted against HOPG yields). Solid line is least squares fit to the solid circles (denoting improved Single Photon yields plotted against $\mathrm{K} \alpha$ Imager yields.

By comparing all three of these $\mathrm{Cu} \mathrm{K} \alpha$ diagnostics a cross-calibration was completed by King et al.[14]. As the data in that paper were obtained in the same experiment, the same cross-calibration was applied to this analysis and is shown in Fig. 5.2. In the cross-calibration, an absolute $\mathrm{K} \alpha$ yield was obtained from single photon counting by summing the number of pixels contained in the 
K $\alpha$ peak. CCD quantum efficiency, single hit probability, filter attenuation and solid angle subtended by the detecting surface, as well as diagnostic view angle and opacities were accounted for to give $\mathrm{Cu} \mathrm{K} \alpha$ photons/sr emitted in the direction of the single photon counting camera. Relative K $\alpha$ line intensities were obtained from the HOPG after integration, background subtraction and opacity correction to also give a total $\mathrm{K} \alpha$ count. The HOPG data were plotted against associated absolute $\mathrm{K} \alpha$ yields from the single photon counting camera to obtain a calibration of the HOPG instrument. A scaling factor was determined as the slope of the linear least squares fit. The HOPG yields were made absolute using this factor, then replotted against the integrated signals from the $\mathrm{Cu} \mathrm{K} \alpha$ imager. A scaling factor for absolute $\mathrm{K} \alpha / \mathrm{sr} / \mu \mathrm{m} / \mathrm{PSL}$ (PhotoStimulated Luminescence values read out by the image plate scanner) was then derived for associated $\mathrm{K} \alpha$ levels corresponding to counts

on the image plates. The statistical error for this scaling factor is estimated to be $20 \%$, with an absolute accuracy error of $50 \%$. The uncertainties of the method and calibrations are discussed in detail in the King et al.[14] paper.

Two extreme ultraviolet (XUV) imagers[89] were also employed at two different photon energies (256 eV and $68 \mathrm{eV}$ ) to record the heating of the nail-wire target. The XUV diagnostics utilized a spherical multilayer mirror imaging at near-normal incidence, with a radius of curvature of $0.5 \mathrm{~m}$, placed approximately $0.27 \mathrm{~m}$ away from the target to capture the Planckian emission. Each channel was paired with a Princeton Instrument PI-SX:1300 CCD with 1300 x 1340 pixels of $20 \mu \mathrm{m}$ size resolution and a polyimide/aluminum filter, viewing at $43^{\circ}$ to the nail-wire axis rear-side.

\subsection{Experimental Results and Analysis}

\subsection{1 $\quad \mathrm{K} \alpha$ Emission and Bulk Heating}

Fig. 5.3 illustrates the intense emission seen from the nail head and the decaying brightness down the wire as displayed by the $\mathrm{Cu} \mathrm{K} \alpha$ crystal imager. The majority of the $\mathrm{K} \alpha$ production occurs in the head.

Absolute $\mathrm{K} \alpha$ yields were determined for the wire portion of the nail-wire tar- 


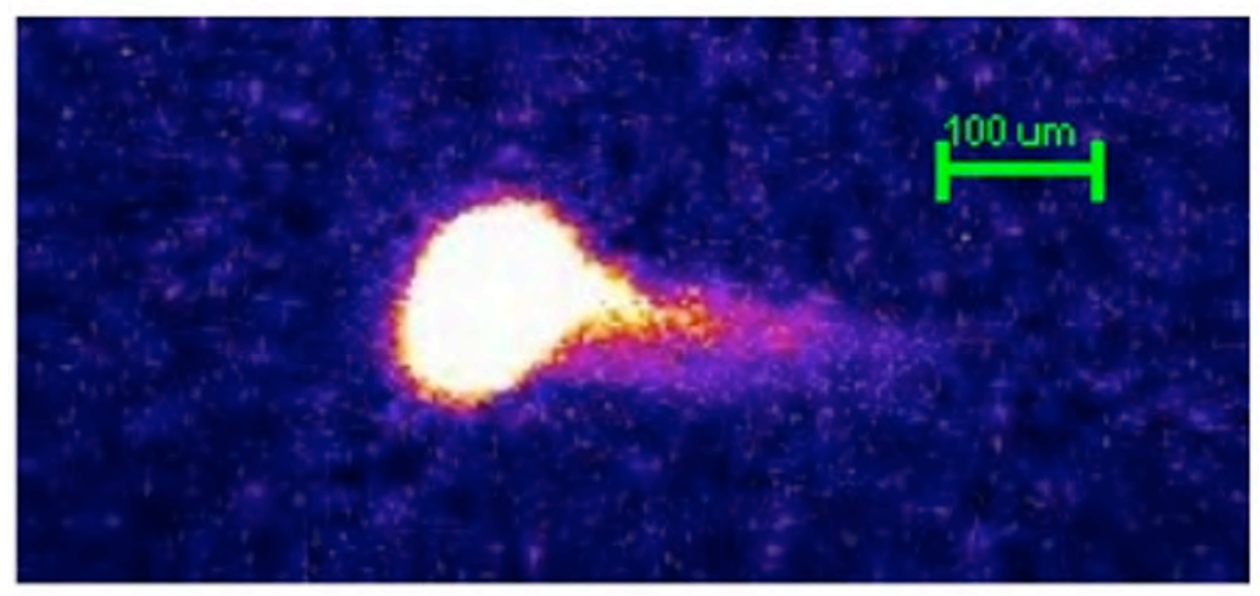

Figure 5.3: $\mathrm{Cu} \mathrm{K} \alpha$ emission from the nail-wire target shown with enhanced color scale.

get. Because the underlying physics of the hot electron transport within the nail head was difficult to analyze due to its hemispherical geometry, only the electron transport within the wire itself is considered in this analysis. The full target (nail head and wire) was composed of one continuous piece of copper, and thus, all of it emitted $\mathrm{Cu} \mathrm{K} \alpha$ radiation. In analysis, the start of the wire was determined by taking a lineout along the wire, and across the nail head. This lineout was of a width large enough to encompass all emission from the wire, and summed the emission transversely at each axial point. Characteristic of the lineout was a location of peak emission from the head, followed by a steep fall-off, followed by a slower quasi-exponential fall-off, where this last portion is a distinctive feature of $\mathrm{K} \alpha$ emission from a wire. The point at where this quasi-exponential decay began was identified in the data to be the beginning of the wire as shown in Fig. 5.4 (0 $\mu \mathrm{m}$ on the abscissa).

Absolute $\mathrm{K} \alpha$ yields were determined for the wire portion of the nail-wire target based on the $\mathrm{Cu} \mathrm{K \alpha}$ crystal image. A lineout integrated transversely along the wire axis was corrected for view angle and opacity. It was assumed that the $\mathrm{K} \alpha$ energy density was homogeneous within a radial cross-section of the wire, and the $22 \mu \mathrm{m} \mathrm{1/e}$ attenuation length of $8048 \mathrm{eV}$ radiation in copper was accounted for in the opacity correction for the $\mathrm{Cu} \mathrm{K} \alpha$ crystal imager line-of-sight. This correction 


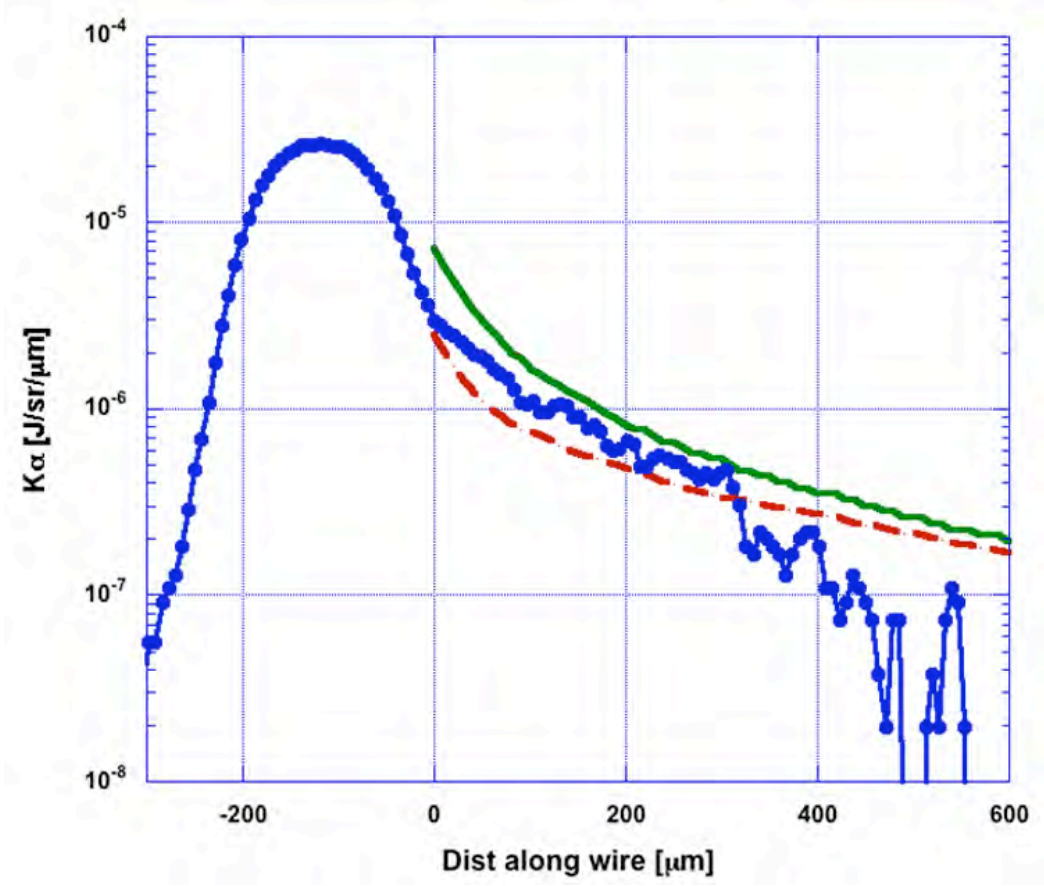

Figure 5.4: $\mathrm{K} \alpha / \mathrm{sr} / \mu \mathrm{m}$ for the nail-wire: experimental (connected dotted line), $1 \mathrm{D}$ numerical model fit to experimental data (dashed), 1D numerical model inferred total $\mathrm{K} \alpha$ emission profile (before any temperature reduction) (solid). The wire to nail head junction is at $0 \mu \mathrm{m}$ on the abcissa scale.

was applied to the observed flux to give the total $\mathrm{K} \alpha$ originating within the wire.

The hot electron transport was modeled using a 1D resistive transport numerical code[66] where the hot electrons were described by a single temperature Maxwell-Boltzmann distribution injected axially into the wire. The code ignored magnetic field effects and electron scattering, but accounted for electron resistive inhibition and the physics was otherwise similar to (but not as complete as) Fokker-Planck and hybrid PIC transport codes. The model assumed full current neutralization by cold background electrons. In small increments along the wire, the loss of electron kinetic energy to binary collisions (calculated using the stopping power of electrons in hot plasmas) and Ohmic energy losses $\left(j^{2} \eta\right.$ heating of the material via the return current drawing kinetic energy from the hot electrons) was calculated together with collisional excitation of $\mathrm{K} \alpha$. Temperature in each step was determined from the specific energy deposited using the SESAME equation of 
state tables. Resistivity of the material was approximated from a fit to the Lee and More electron conductivity model for hot plasmas[90]. The local atomic number $Z$ was also predicted from the SESAME tables. The code generated axial K $\alpha$ and temperature profiles along the wire.

The modeled K $\alpha$ profile was matched to the experimental axial emission profile (corrected for view angle and opacity) by varying the simulation input parameters of injected laser energy (i.e., coupling efficiency) and initial hot electron temperature $\left(T_{h o t}\right)$. These two free parameters could be varied in this forward calculation to change the peak $\mathrm{K} \alpha$ value and the slope of the model profile. Additionally, this transport model accounted for the loss in signal due to the shifting of the $\mathrm{K} \alpha$ line out of the bandwidth of the $\mathrm{Cu} \mathrm{K} \alpha$ crystal with increasing target temperature. The earlier cross calibration of the HOPG and integrated $\mathrm{K} \alpha$ images produced a notional absolute calibration of the imager, but it is known that the imager underrecords $\mathrm{K} \alpha$ due to the temperature effect (quantified by Akli et al.[66] by using the population kinetics model FLYCHK[91], see Fig. 3.12). Therefore the deduced K $\alpha$ photons per integrated image count were overestimated by some initially unknown factor $R$. The Akli model calculated the absolute $\mathrm{K} \alpha$ profile for assumed injected electrons as described above, then calculated the reduction of the profile based on the temperatures it predicted to give the profile expected to be recorded $\alpha$ profile to the HOPG; and it follows that both the Akli calculated detected profile and the original experimental profile also have to be scaled down by this same factor $R$. The process was reiterated until the solutions converged on where the Akli true $\mathrm{K} \alpha$ profile integrates to match the HOPG absolute $\mathrm{K} \alpha$ number, and the reduced Akli profile fits the experimentally recorded one.

The absolute $\mathrm{K} \alpha$ emission profile derived in this fashion had a peak intensity of $7.1 \times 10^{-6} \mathrm{~J} / \mathrm{sr} / \mu \mathrm{m}$ at the start of the wire, and an exponential fall off of approximately $60 \mu \mathrm{m}$ scale length along the wire. Shown in Fig. 5.4 are the experimental data (blue connected dotted line), and both the simulated $\mathrm{K} \alpha$ image profile fitted to the experimental data (red dashed line) and the inferred total $\mathrm{K} \alpha$ emission profile after the correction for diagnostic underestimation of the signal due to the temperature shift (green solid line). 
Also based on the 1-D code, the laser energy converted into electrons injected in to the wire was approximately $10.35 \mathrm{~J} \pm 5 \mathrm{~J}$. With an initial injection laser energy of $309 \mathrm{~J}$, this gave a coupling efficiency into hot electrons of approximately $3.3 \% \pm 1.7 \%$. It is of interest to note that this value of $3.3 \%$ is the energy coupled into the wire alone; obviously, as evidenced by the raw $\mathrm{K} \alpha$ images, considerable energy was deposited within the nail head itself. Fitting to the model profile in the 1D code also provided estimates of the average electron temperature in the wire. $\mathrm{T}_{\text {hot }}$ was determined to be approximately $620 \mathrm{keV} \pm 125 \mathrm{keV}$. The maximum $\mathrm{T}_{\text {cold }}$ (background electron mean temperature) at the start of the wire was approximately $68 \mathrm{eV} \pm 34 \mathrm{eV}$, and also decreased exponentially along the wire. The accuracy of all the numbers derived from this 1D transport calculation is limited by the accuracy of the absolute calibration to which the experimental data was scaled. Because both the conversion efficiency and the temperature rise of the target are proportional to absolute yield, we estimate the errors in their calculation to be \pm $50 \%$. The electron temperature is directly proportional to the square root of the absorbed laser intensity and the profile slope. Thus, the majority of the error in deriving the electron temperature lies again in how closely we can estimate the fraction of absorbed energy, which is subject to the same $\pm 50 \%$ accuracy in the absolute yield. Convolving in the error from the slope profile translates to an error bar of $\pm 20 \%$.

Both the experimental and model-predicted hot electron penetration scale lengths are much shorter than the collisional stopping range of the hot electrons $(\sim 370$ $\mu \mathrm{m}$ for a $600 \mathrm{keV}$ electron), implying that the fast electron penetration is limited by the Ohmic potential[42].

\subsubsection{XUV Emission and Surface Heating}

Figure 5.5 shows extreme ultraviolet images of the nail-wire target at the 256 $\mathrm{eV}$ and $68 \mathrm{eV}$ photon energies. Prominent in the $256 \mathrm{eV}$ image is limb brightening, where XUV emission at the edge of the image is more intense than at the center. The obvious limb brightening seen in the $256 \mathrm{eV}$ image implies preferential heating concentrated near the surface of the wire. However, limb brightening is not 

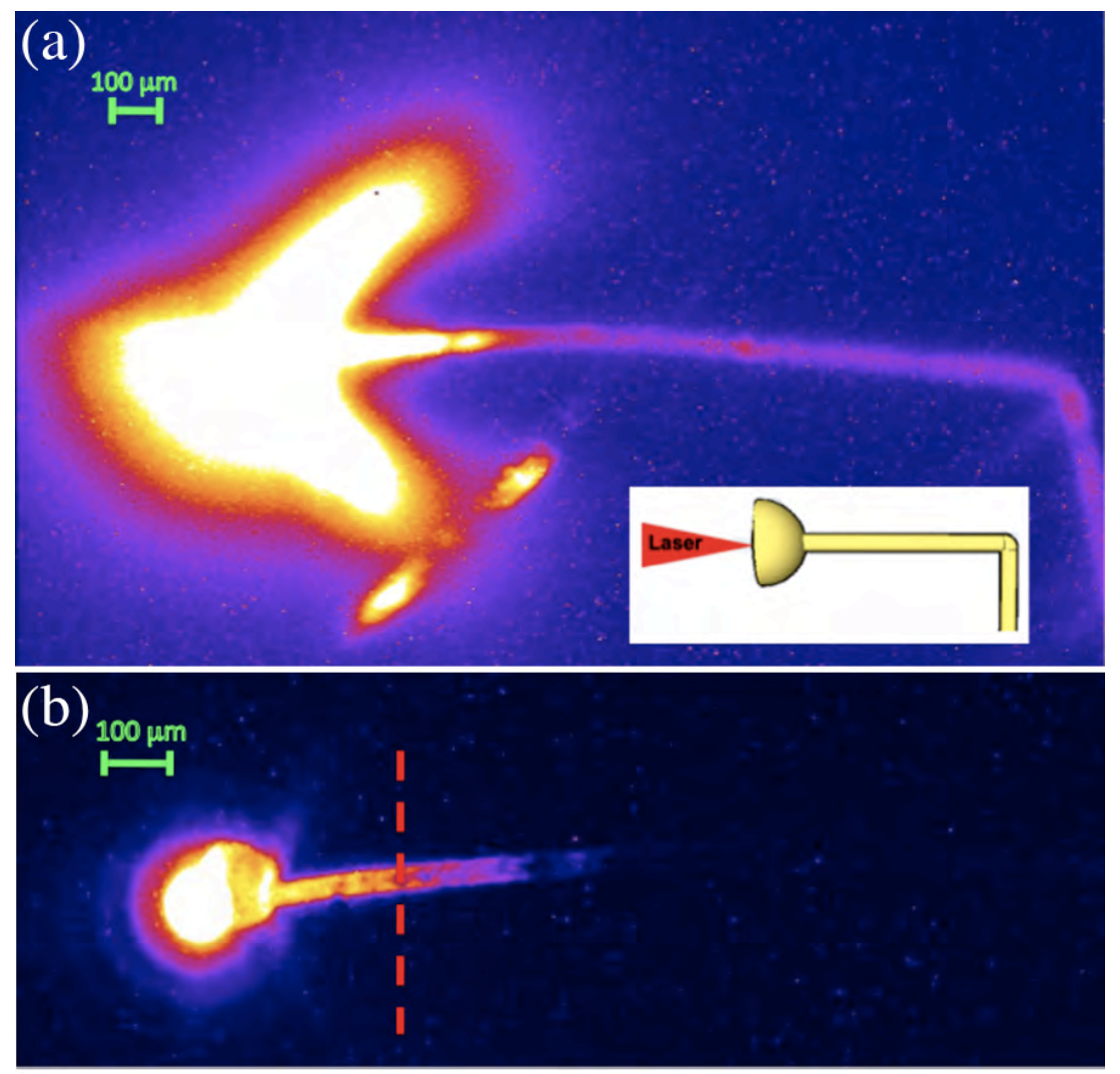

\section{(c)}

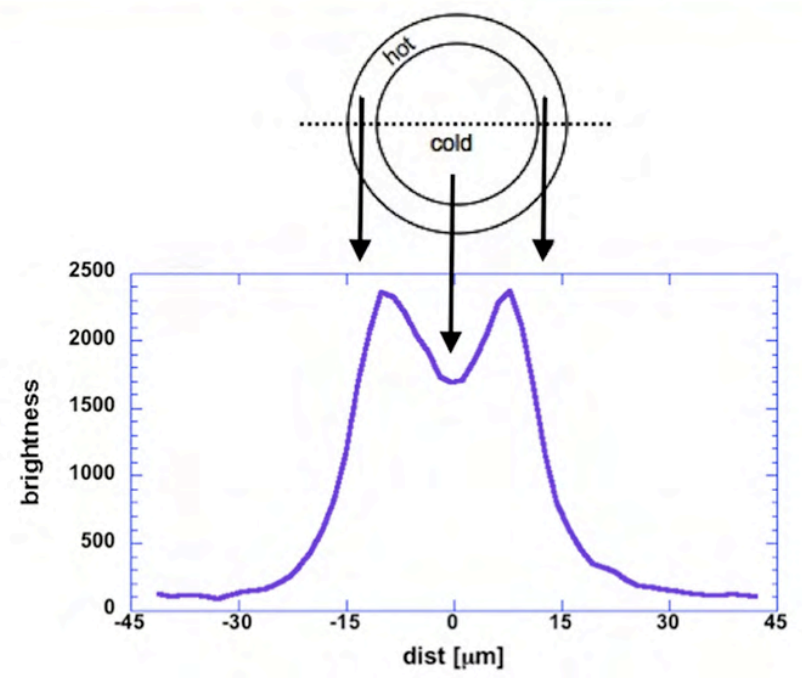

Figure 5.5: (a) Extreme ultraviolet image (at $68 \mathrm{eV}$ ) of the nail-wire. Emission can be seen all along the nail-wire, and even around the bend. Inset: Schematic showing illumination geometry. (b) Extreme ultraviolet image (at $256 \mathrm{eV}$ emission) of the nail-wire. Dotted line denotes position of lineout in (c) Transverse lineout of wire in $256 \mathrm{eV}$ XUV image which displays prominent limb brightening 
observable in the $68 \mathrm{eV}$ image. This suggests that the thickness of the emitting surface layer is less than the attenuation length of the $256 \mathrm{eV}$ radiation, but greater than the attenuation length of the $68 \mathrm{eV}$ emission. Based on the 0-D atomic spectroscopy code FLYCHK[91], in the temperature range of $30-200 \mathrm{eV}$ (a reasonable range of temperatures for the wire surface[87]) with the mass density specified for solid $\mathrm{Cu}$, the attenuation length for $256 \mathrm{eV}$ radiation is between $40-130 \mathrm{~nm}$, and for $68 \mathrm{eV}$ radiation is between $5-20 \mathrm{~nm}$. Thus, we expect this heated surface layer to have an areal mass on the order of $40-130 \mathrm{~nm}$ of solid $\mathrm{Cu}$. Also of note, the limb brightening becomes more prominent further down the wire away from the nail-head, indicating that the heating is more uniform and the opacity of the hot layer is greater nearer to the nail head.

The time-integrated XUV images in Figure 5.5 also show thermal emission far down the wire, and in the case of the $68 \mathrm{eV}$ image, the emission even extends around the wire bend-a distance of over $1.3 \mathrm{~mm}$. This is consistent with the observation of a directional electron jet in vacuum emitted along the axis of a cone fiber target with a $1 \mathrm{~mm}$ long carbon fiber[86].

The $68 \mathrm{eV}$ XUV image also shows an expanding plasma halo that wraps around the nail head, and includes plasma jets at the wire bend and where the nail head meets the wire. These plasma jets are a manifestation of the collision of hot plasma from intersecting surfaces.

\section{4 e-PLAS Simulations}

The 2-D e-PLAS simulation code based on implicit-moment/hybrid techniques[92] was used to model the transport of the relativistic electrons in these nail-wire targets[93]. Other modeling of wire geometries was done in references [87] and [85]. However, this set of simulations is novel in that it was performed close to experimental conditions: the target was modeled at full scale and electron injection in the model was based on the experimental laser parameters. Calculations were performed for particles in a 2-D cylindrical geometry, with simulation runs spanning 2 ps. The laser pulse was a normally incident $1.06 \mu \mathrm{m}$ beam in a 10 
$\mu \mathrm{m}$ FWHM gaussian spatial envelope with a flat temporal pulse terminating at 1 ps, with a peak intensity of $1.7 \times 10^{20} \mathrm{~W} / \mathrm{cm}^{2}$. A density gradient with a $40 \mu \mathrm{m}$ density ramp varying from $\sim 10^{21}$ to $10^{18}$ electrons $/ \mathrm{cm}^{3}$ preceded the solid target and represented the effect of the ASE prepulse[59]. The assumed laser absorption was 20\% into an isotropic, relativistic Maxwellian hot electron distribution injected at the critical density, with hot electron temperature locally determined by an empirical scaling based on experimental data detailed by Beg et al.[32] The target material was modeled as copper with an average effective atomic number of $Z=15$ and an initial temperature of $100 \mathrm{eV}$. The simulation was performed with 50 radial cells (each $1 \mu \mathrm{m}$ wide) and 200 axial cells (each $1.5 \mu \mathrm{m}$ wide). The modeled target had a $45 \mu \mathrm{m}$ radius, $50 \mu \mathrm{m}$ deep hemispherical head and a wire diameter of $20 \mu \mathrm{m}$, and was truncated at $175 \mu \mathrm{m}$. For numerical self-consistency purposes, at vacuum-material interfaces, the sharp boundary was smeared over several cells. As the hot electrons were transported down the wire, they drew resistive cold-fluid return currents (where the energy is carried by the bulk, slow-moving electrons) and generated self-consistent $\vec{E}$ and $\vec{B}$ fields.

Fig. 5.6 shows the hot particle density at 2 ps (i.e., 1 ps after the end of the laser pulse), and it can be seen that surface currents of hot electrons run toward the head edges as well as axially down the wire. The on axis lineout of Fig. 5.6(b) shows that the majority of hot electrons remain in the nail head. The hot electron density in the wire section (which starts at $100 \mu \mathrm{m}$ on the axial scale) varied from $4 \times 10^{20}$ to $1 \times 10^{19}$ electrons $/ \mathrm{cm}^{3}$ at the end of the wire. The $1 / e$ falloff for the hot electron density in the wire region was approximately $76 \mu \mathrm{m}$. The cold electron density on axis in Fig. 5.6(b) did not change significantly from the initial solid density value of $10^{24} \mathrm{~cm}^{-3}$ in the $2 \mathrm{ps}$ run, there being insufficient time for a hydrodynamic response. e-PLAS predicted a coupling efficiency into electrons into the wire of approximately $9 \%$, calculated as the sum of the energy in the hot electrons and the energy deposited thermally. The discrepancy between the coupling efficiency predicted by e-PLAS and the $3.3 \%$ predicted by the 1D transport model is explored in the Discussion section.

The 2-D temperature plot of Fig. 5.7(a) provides modeling evidence for the 
(a)

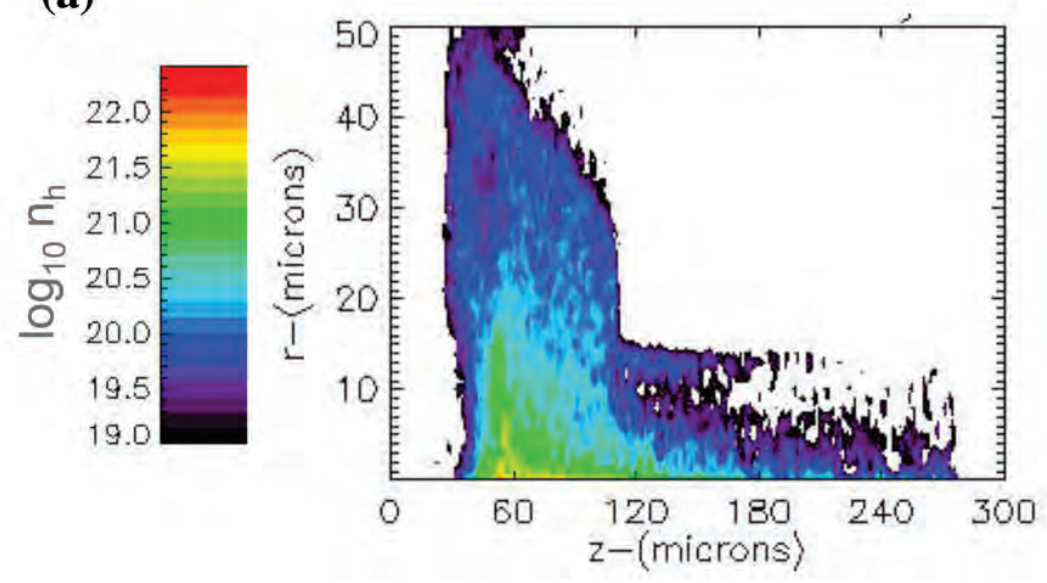

(b)

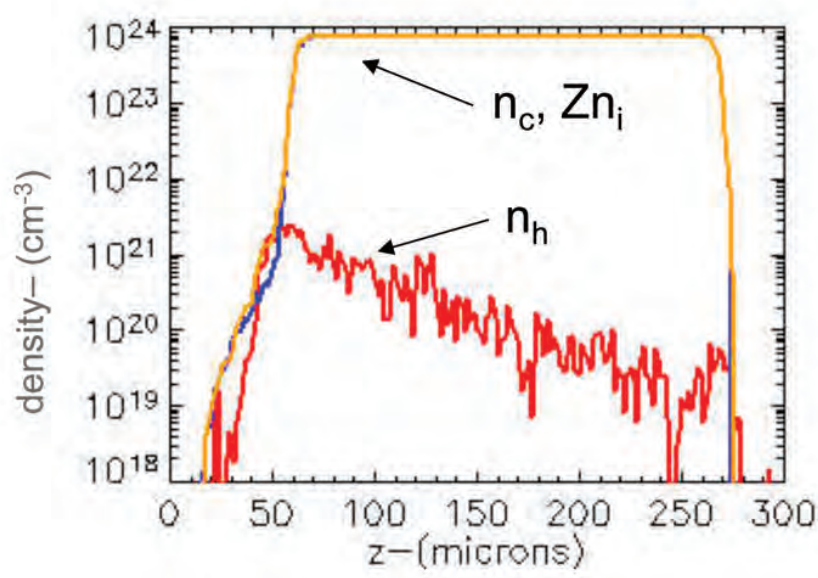

Figure 5.6: (a) e-PLAS hot electron density plot at 2 ps. (b) Lineouts of the transverse averages for $\mathrm{n}_{h}$ (red), $\mathrm{n}_{c}$ (blue), and $\mathrm{Zn}_{i}$ (brown). The $\mathrm{n}_{h}$ profile shows a near-exponential falloff along the full length of the wire. The $1 / e$ length from e-PLAS is $\sim 76 \mu \mathrm{m}$. 
surface heating seen with the XUV images. The cold temperatures show nearly radially uniform $\mathrm{T}_{\text {cold }}$ of $200 \mathrm{eV}$ throughout the bulk of the wire except at the outer surface where the temperature peaks to $3 \mathrm{keV}$. Note that the $1 \mu \mathrm{m}$ radial zone width is too large to resolve the hot surface layer thickness. The computed cold temperature rise in the center of the wire at 2 ps (Fig. 5.7(b)) was $1500 \mathrm{eV}$ at the start of the wire falling to $100 \mathrm{eV}$ after $125 \mu \mathrm{m}$, with a scale length of $50 \mu \mathrm{m}$. The $1500 \mathrm{eV}$ maximum is much higher than was inferred from fitting experimental data with the Alki model and the difference is also covered in the Discussion section.

A transverse profile of the average density across the wire at $\mathrm{z}=120 \mu \mathrm{m}$ (Fig. 5.7(c)) is interesting in that it shows a high density of cold electrons everywhere in the wire except just in the outer few microns where hot electrons dominate. The outer edge is the Debye sheath region, which supports a longitudinal current of fast electrons just outside the wire. The transverse profile of $\mathrm{T}_{\text {cold }}$ (Fig. 5.7(d)) shows that the cold electron temperature profile peaks at a slightly smaller radius than where the hot electrons dominate. This observed higher surface temperature is due to the return current that develops to compensate the hot surface current just outside. As a note, the nearly $20 \mathrm{keV}$ temperature predicted right at the wire edge is unrealistic, and is likely due to the smearing of the density profile over several neighboring cells in the simulation. The smearing gives the outer zones a very low density of plasma, and when a high return current passes through this area, an artificially high amount of Ohmic heating is produced. The predicted $3 \mathrm{keV}$ plateau just to the left of the spike is a probably a more realistic representation of the temperature in the solid density of the target.

Magnetic field contours are shown in Fig. 5.8(a), and an axial profile taken at $\mathrm{r}=13 \mu \mathrm{m}$ is shown in Fig. 5.8(b). Predicted toroidal $\vec{B}$ fields (due to $\nabla T \times \nabla n$ ) are intense at the entry surface, where they are calculated to be approximately 50 MG at 2 ps. These intense localized azimuthal fields were similarly seen in LSP simulations by Wei et al.[87] The $\vec{B}$-field direction between the head and $100 \mu \mathrm{m}$ down the wire indicated a current flow toward the head in the Debye sheath region. This is consistent with a hot electron flow away from the head in the same region 
(a)
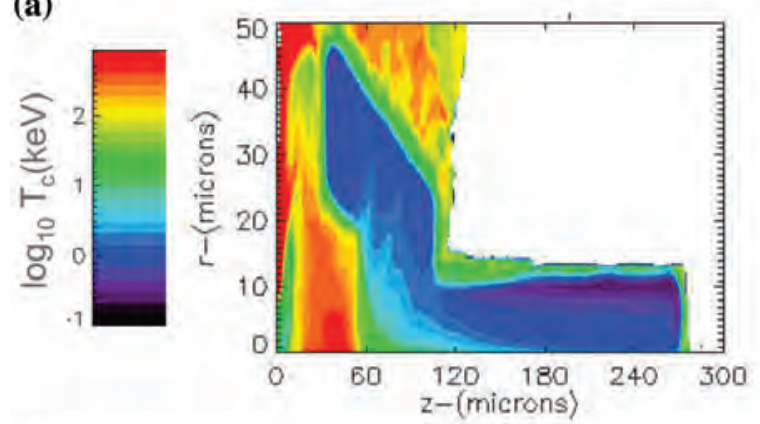

(b)

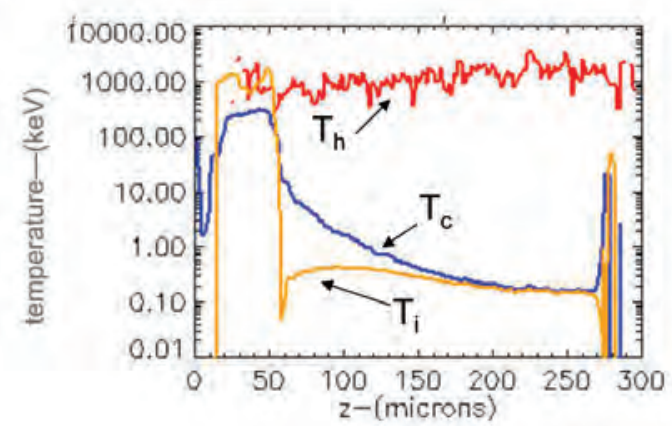

(c)

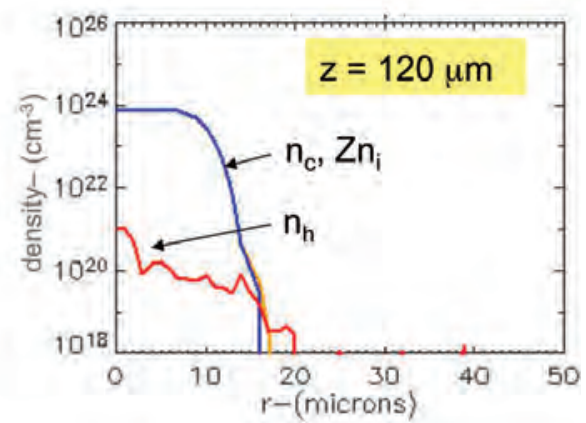

(d)

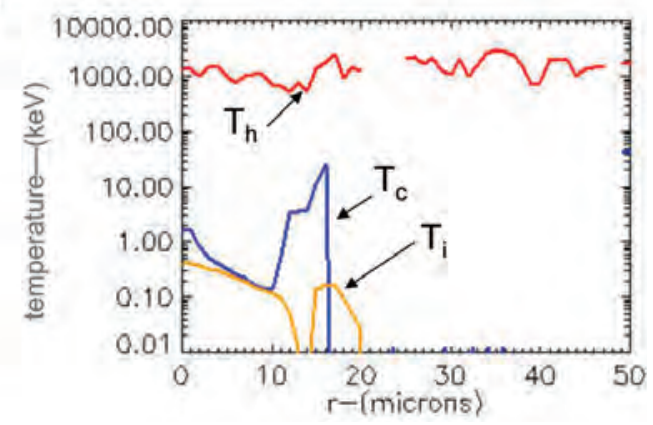

Figure 5.7: (a) e-PLAS thermal (cold) electron contours at 2 ps. Plot displays enhanced surface heating along the edges of the target. (b) Lineouts of the temperature profiles at 2 ps for hot electrons (red), cold electrons (blue), and ions (brown). Beyond $\mathrm{z}=225 \mu \mathrm{m}(125 \mu \mathrm{m}$ along the wire), both the cold electrons and the ions are at a similar temperature of $200 \mathrm{eV}$. (The target starts out with an initial temperature of $100 \mathrm{eV}$.) (c) Transverse average density profiles across the wire at $\mathrm{z}=120 \mu \mathrm{m}$. The hot electron density is seen to extend to a larger radius than the cold electron or ion densities. This correlates to the large flux of hot electrons surfing along the outside edge of the wire. (d) Transverse temperature profiles for the wire at $\mathrm{z}=120 \mu \mathrm{m}$. $\mathrm{T}_{c}$ is seen to peak just at the edge of the wire. 
(a)

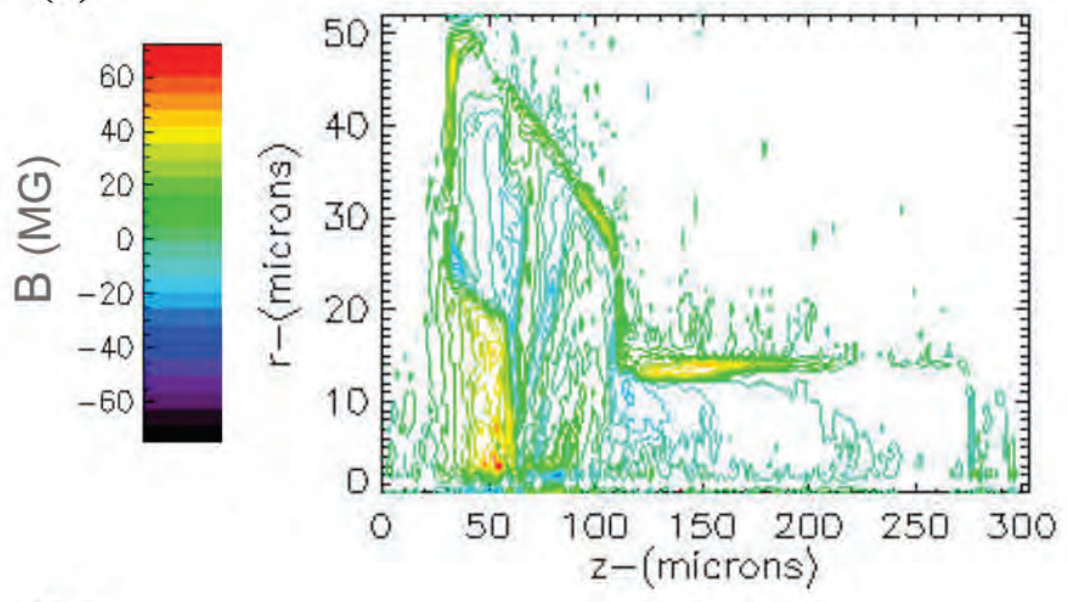

(b)

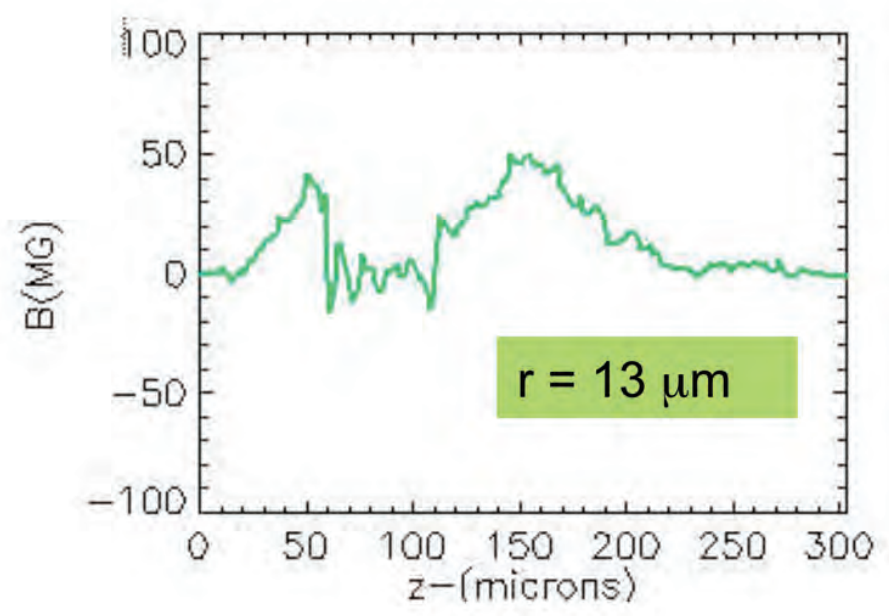

Figure 5.8: (a) e-PLAS magnetic field contours at 2 ps. A strong $\vec{B}$ field is seen all around the nail head, confining electrons. (b) Axial profile of the magnetic field taken at $\mathrm{r}=13 \mu \mathrm{m}$. Fields are largest $(\sim 50 \mathrm{MG})$ near the laser spot and along the wire surface near the head-wire interface. $\vec{B}$ field sign is generally positive, indicating that the dominant hot electron flow is away from the head, while return current lies just inside the wire surface. 
and can be viewed as electrons in the Debye sheath surfing along the wire. The corresponding return current is expected to be within a skin depth inside the wire surface and causes the previously discussed surface heating. Resistive heating was dominant in the bulk of the wire, with axial $\vec{E}$ fields up to $0.02 \mathrm{MeV} / \mu \mathrm{m}$ in the center of the wire, so that over a distance of $100 \mu \mathrm{m}$, the hot electrons lose 2.0 $\mathrm{MeV}$. A transverse cut through the wire also showed the magnitude of $\vec{E}$ fields to be over 10 times larger at the edge of the wire, corresponding to the higher current density along the wire surface.

Simulations in which the resistivity and magnetic fields were switched on and off $[92,93]$ demonstrated that the reduced penetration of the hot electrons in the wire could be attributed to these effects. When both the $\vec{B}$ fields and resistivity were turned off, a strong, directed beam of electrons could be seen penetrating very far down the wire (beyond the $250 \mu \mathrm{m}$ simulation box). However, with both effects on, a dense concentration of hot electrons was trapped in the nail head and in the wire with the axial variations present as previously mentioned. The hot electron densities and heating in the wire also fell off due to the electric field retardation in a manner closely resembling that seen in experiment.

\subsection{Discussion}

The results described here show a qualitative agreement between the experimental data and the e-PLAS modeling, which support the arguments for a dominant Ohmic inhibition effect on the hot electrons within the bulk of the wire while the surfing hot electron current in the Debye sheath leads to heating of a thin surface layer.

It should be noted, however, that the thickness of this heated layer predicted by e-PLAS is influenced by the $1 \mu \mathrm{m}$ zone width of the simulation. It is expected that should the resolution of the simulation be increased, the heated layer would converge to a thickness on the order of a skin depth. However, due to the boundary layer smearing alluded to earlier, e-PLAS cannot correctly compute the level of surface heating in the sheath region, but serves to give qualitative evidence of 
the existence of this hot layer. The situation in the hot surface layer is further complicated by radial acceleration of protons due to hot electron pressure and hydrodynamic expansion due to cold electron pressure. Thermal conduction also broadens the heated layer. These are effects that are not modeled by e-PLAS.

Thermal cold electron temperature rises predicted by e-PLAS (1500 eV at the start of the wire) are much higher than those predicted by the 1D transport code $(68 \mathrm{eV})$. As the coupling efficiency predicted by e-PLAS is approximately a factor of 3 higher than the 1D transport code (9\% versus $3.3 \%$ ), this corresponds to a three times higher electron flux. Ohmic heating, which goes as $j^{2}$, would then be almost a factor of 10 greater, yielding temperatures also a factor of 10 greater. The remainder of the temperature discrepancy can be attributed to the different equation of state models (SESAME tabulated values for the 1D transport code, ideal gas for e-PLAS). Further, in e-PLAS, a reflecting boundary condition existed at the wire end allowing the electrons to bounce around until all their energy was deposited, whereas electrons in the 1D transport code made only one pass through the wire and then exited out the end. While this has been accounted for in the interpretation of conversion efficiency, this effect also contributes to the low temperatures predicted by the 1D transport model.

A parallel study of cone-coupled wire targets[14, 94] was done in the same experiment with identical laser characteristics and diagnostic setups. These conewire targets used a $\mathrm{Cu}$ wire of a similar length and diameter glued to an $\mathrm{Al}$ cone, rather than drawn directly from a hemispherical head. The characteristics of bulk transport in the two types of targets were very similar. Emission scalelengths are within $25 \%$, and predicted electron temperature, $\mathrm{T}_{\text {hot }}$, within $10 \%$. However, the laser to wire hot electron coupling efficiency was found to be slightly higher in the cone case (5\% vs. $3.3 \%$ in the nail-wire case), suggesting advantages of the cone geometry for FI applications. Interestingly, while XUV imaging of the nail-wire showed considerable XUV emission from the wire, similar images of the cone-wire show no detectable XUV emission from the wire portion of the target. We attribute this to lateral transport of the hot electrons along the front edge and around the nail head and then subsequent surfing along the wire surface. In the case of the 
cone-wire target, hot electrons would have had to travel all the way back up the inside surface of the cone $\left(\sim 1 \mathrm{~mm}\right.$ in length) before making a $360^{\circ}$ turn to reach the outside of the cone.

The strong, localized surface magnetic fields observed in the e-PLAS simulations were similarly seen by Wei et al.[87] to be on the order of 50 - $100 \mathrm{MG}$ at the front of the target. These fields, which tend to trap the lower energy electrons and inhibit their propagation into the target, are also responsible for the lateral propagation of these electrons along the surface of the target.

In conclusion, the transport of energy by laser-generated fast electrons in a nail-wire geometry has been investigated experimentally and numerically. Strong Ohmic inhibition has been observed in the nail-wire targets similar to that in cone-wire targets. Coupling into the wire in the nail-wire geometry is somewhat lower than observed in cone-wire targets. One significant difference between the two targets is the prominent surface heating is observed in the case of nail-wire targets.

Chapter 5, in full, is a reprint of the material as it appears in T. Ma, Physics of Plasmas 16, 112702 (2009). The dissertation author was the primary investigator and author of this paper. 


\section{Chapter 6}

\section{Effects of Preplasma on Hot}

Electron Coupling and

Propagation in Cone-Attached

\section{Wire Targets}

\subsection{Introduction}

The reentrant cone approach to fast ignition remains one of the most attractive because of the potential to effectively collect and guide the laser light into the cone tip and direct energetic electrons into the high density core of the fuel. However, in the presence of a preformed plasma, the laser energy can be largely absorbed before it can reach the cone tip.

Laser prepulses, or the pedestal of energy before the main pulse arrives, are inherent in short pulse, high intensity lasers, due to amplified superfluorescence and spontaneous emission. Existing petawatt lasers which can deliver up to $1 \mathrm{~kJ}$ and $I$ $\geq 10^{20} \mathrm{~W} / \mathrm{cm}^{2}$ will often have intensity contrast levels between $10^{-6}-10^{-8}$. Such an ASE intensity is above the ionization threshold of most materials $\left(10^{10}-10^{11}\right.$ $\mathrm{W} / \mathrm{cm}^{2}$ ), meaning there is more than sufficient energy to modify the solid target and create a substantial plasma before the main pulse arrives. This preplasma 
will expand outwards from the surface of the solid target, forming a region of lowdensity plasma the main pulse must propagate through before depositing its energy at the critical surface (which has since been shifted out from the solid surface). The absorption of the laser is therefore severely affected, and the hot electron generation and its energy spectrum and divergence is modified.

Full scale fast ignition laser systems of $100 \mathrm{~kJ}$ are envisioned to have prepulse energies ranging between $100 \mathrm{~mJ}$ to $1 \mathrm{~J}$. Consequently, the issue of preplasma formation and its effect on conversion of laser light to hot electrons, the subsequent transport characteristics of those electrons, and the resulting requirements for maximum allowable prepulse this may put on the laser system are imperative issues facing fast ignition that must be understood.
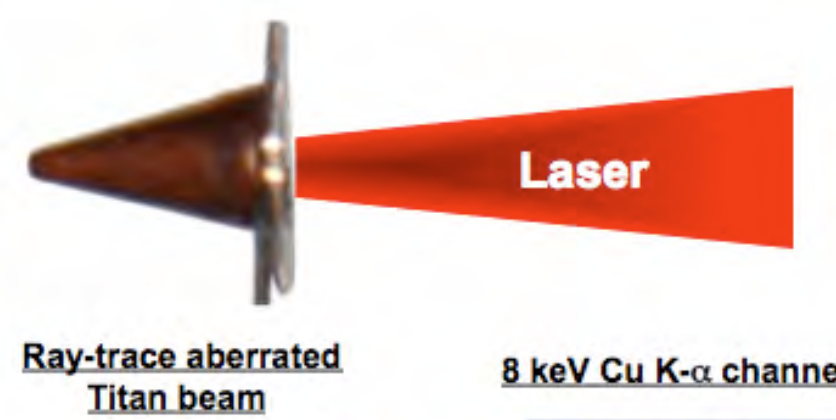

8 keV Cu K- $\alpha$ channel
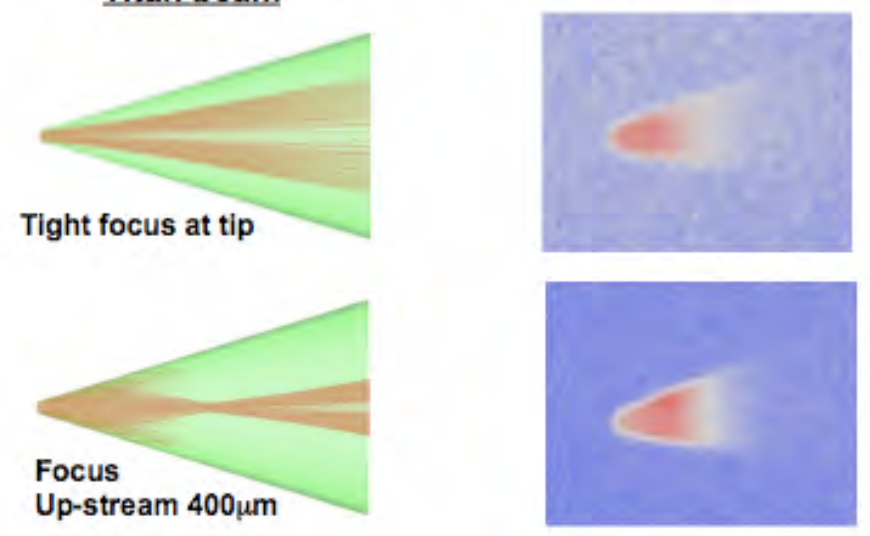

Figure 6.1: Even under varied laser focusing conditions (ray tracing shown here for the case of tight focus at the cone tip, and focusing to a point $400 \mu \mathrm{m}$ before the cone tip), the patterns of $\mathrm{Cu} \mathrm{K} \alpha$ fluorescence from the cone target are very similar. 
Work by Van Woerkom et al.[95] in 2008 hinted at the prejudicial role of preplasma in preventing laser energy from reaching the tip of the fast ignition cone. Cones were irradiated with the Titan laser, under varying laser focal conditions, and 2-D spatial $\mathrm{K} \alpha$ imaging was performed. The cones were composed of $\mathrm{Cu}$ walls $25 \mu \mathrm{m}$ thick, $800 \mu \mathrm{m}$ in length, with a $30 \mu \mathrm{m}$ inner tip diameter, and a $30^{\circ}$ full opening angle. Shots were taken with the laser injected at tight focus (best focus) on the cone tip, then defocused to $400 \mu \mathrm{m}$ and $800 \mu \mathrm{m}$ beyond the cone tip, and $400 \mu \mathrm{m}$ before the cone tip. Prepulse levels were around $15 \mathrm{~mJ}$ for every shot. Although defocusing corresponded to a lower laser intensity at the cone tip compared to the tight focus case, the pattern of $\mathrm{Cu} \mathrm{K \alpha}$ fluorescence did not vary considerably under these different focusing conditions. In every case, the distribution of emission was similar, extending from the cone tip to $300 \mu \mathrm{m}$ distance along the cone walls.

Such a lateral extent of emission was larger even than predicted by ray tracing of the different focus geometries, and larger than the lateral spread seen for equivalent flat foils. It was hypothesized, then, that the laser-cone interaction was actually dominated by the preplasma filling of the cone. Hot electrons excited by the main pulse could freely diffuse in the conducting plasma region created by the prepulse, yielding the large region of $\mathrm{K} \alpha$ fluorescence. See Fig. 6.1.

A concurrent study by Baton et al.[96] measured coupling into targets with and without cones attached to assess the benefits of using a cone. Somewhat surprisingly, they found that the coupling from the laser to a planar $\mathrm{Al}(20 \mu \mathrm{m}) /$ $\mathrm{Cu}(20 \mu \mathrm{m}) / \mathrm{Al}(20 \mu \mathrm{m})$ foil irradiated directly, was much higher than when the same foil was coupled to a $30^{\circ}$ full angle, $40 \mu \mathrm{m}$ wide open tip cone (see Fig. 6.2 for a picture of the cone-foil target). Fig. 6.3 shows the $\mathrm{Cu} \mathrm{K} \alpha$ images when a $1 \omega_{0}, 0.4 \mathrm{ps}, 5 \times 10^{17} \mathrm{~W} / \mathrm{cm}^{2}$ laser pulse with a $2 \mathrm{~ns}$ ASE pedestal, focused $300 \mu \mathrm{m}$ before the target plane, was introduced onto a target without (a) and with (b) a cone. As can be seen in the figure, the $\mathrm{K} \alpha$ spot is far more intense without the 


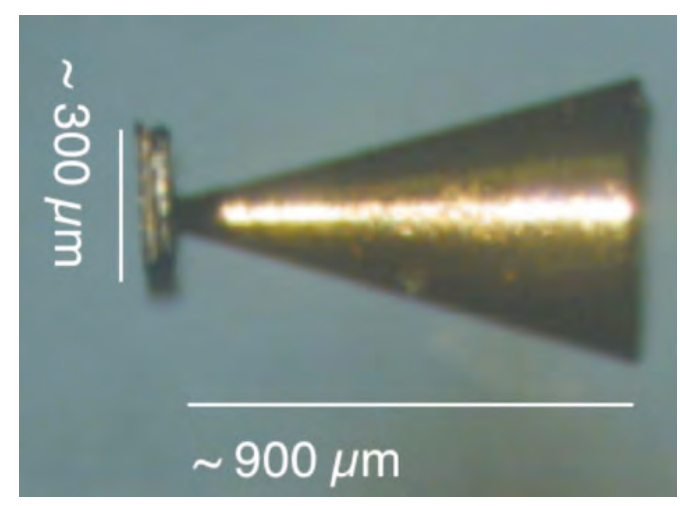

Figure 6.2: Typical cone-attached target used in the experiment by Baton et al. The Au cone has a $20 \mu \mathrm{m}$ aperture and $30^{\circ}$ total angle. The cone tip is fixed to a square $300 \times 300 \mu \mathrm{m}^{2}$ planar target. Figure taken from Baton et al., Physics of Plasmas (2008)[96].

cone in (a), and was quantitatively measured to be greater by a factor of 6.5 .

These shots were then repeated, at $2 \omega_{0}$ laser frequency. Because the initially $1 \omega_{0}$ laser must be sent through a nonlinear doubling crystal to become $2 \omega_{0}$, the energy contrast can be improved to better than $10^{10}$, meaning essentially no prepulse. However, this also reduces the maximum available laser intensity to about $20 \%$ what it was in $1 \omega_{0}$, and the corresponding $I \lambda_{0}^{2}$ drops by $75 \%$. All $2 \omega_{0}$ shots were performed under the best focusing conditions. In this case, the measured $\mathrm{K} \alpha$ fluorescence was comparable from planar foils with and without cones attached (see Fig. 6.4).

As discussed in detail by Baton et al., it was conjectured that in the case of irradiation by the $1 \omega_{0}$ laser, the cone-induced decrease in $\mathrm{Cu} \mathrm{K} \alpha$ yield is likely due to a confinement of the preplasma in the cone geometry as compared to a planar target. Whereas for a planar target the preplasma has infinite room to transversely expand, with a cone, the walls restrict the transverse expansion and the preplasma fills the cone. When the ASE level was substantially reduced by frequency doubling the pulse, large-scale preplasma formation was prevented, and comparable $\mathrm{K} \alpha$ signals were seen with and without the cone, confirming the adverse consequence of prepulse in cone. 

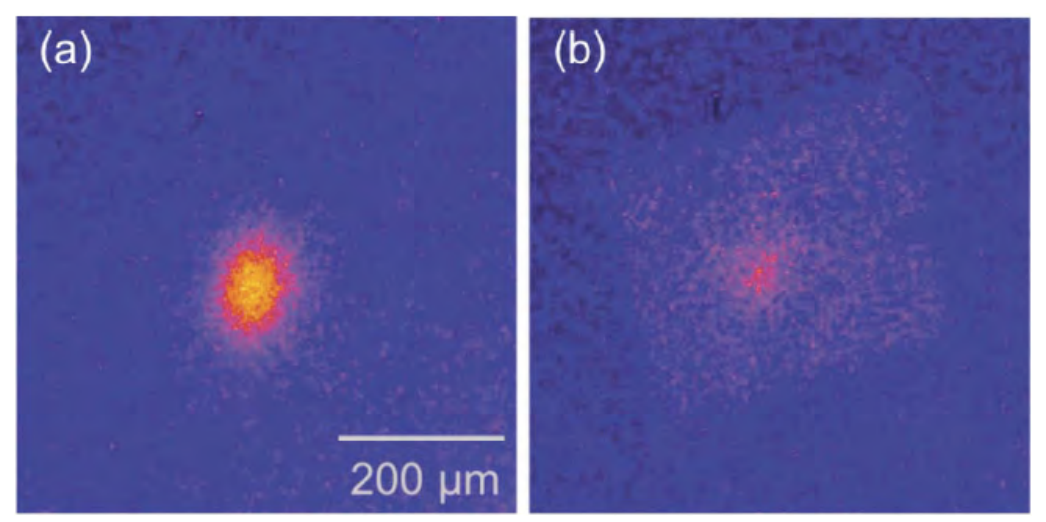

Figure 6.3: $\mathrm{K} \alpha$ images of $\mathrm{Al}(20 \mu \mathrm{m}) / \mathrm{Cu}(20 \mu \mathrm{m}) / \mathrm{Al}(20 \mu \mathrm{m})$ targets irradiated with a $1 \omega_{0}, 0.4 \mathrm{ps}, 300 \mu \mathrm{m}$ defocused, $5 \times 10^{17} \mathrm{~W} / \mathrm{cm}^{2}$ laser with a $2 \mathrm{~ns}$ ASE prepulse pedestal. In (a), the foil was irradiated directly, while in (b), a Au cone of $30^{\circ}$ opening angle, $40 \mu \mathrm{m}$ wide tip was attached to the foil. Both images use the same color scale. Figure taken from Baton et al., Physics of Plasmas (2008)[96].

To quantitatively investigate laser absorption as a function of laser contrast, MacPhee et al.[97] imaged K $\alpha$ emission from the walls of freestanding copper cones irradiated with the Titan $150 \mathrm{~J}, 0.7$ ps laser, preceded by two different levels of prepulse. Fig. 6.5 are the two $\mathrm{Cu} \mathrm{K} \alpha$ images showing the distribution of energy in the cone walls in the instance of (a) $7.5 \mathrm{~mJ}$ of prepulse and (b) $100 \mathrm{~mJ}$ of prepulse. In the low prepulse case, the emission peaks at the tip and is concentrated within $200 \mu \mathrm{m}$ of the tip. In the high prepulse case, the emission peaks $200 \mu \mathrm{m}$ from the cone tip and extends back an additional $300 \mu \mathrm{m}$.

The data was modeled by first performing 2-D simulations with the HYDRA radiation-hydrodynamics code to calculate the preformed plasma profile inside the cone target using the experimentally measured laser prepulse intensity distribution. These plasma density profiles were used as the input to 2-D particle-in-cell simulations performed with the PSC code[98]. The spatial profile of the high intensity pulse was simulated in the PIC calculation using a reconstructed beam phase front based on the measured focal spot from an on-shot equivalent plane monitor.

The simulations were then compared to the experimental data by computing the energy density of (K $\alpha$-producing) electrons. Fig. 6.6 shows that the simulations 

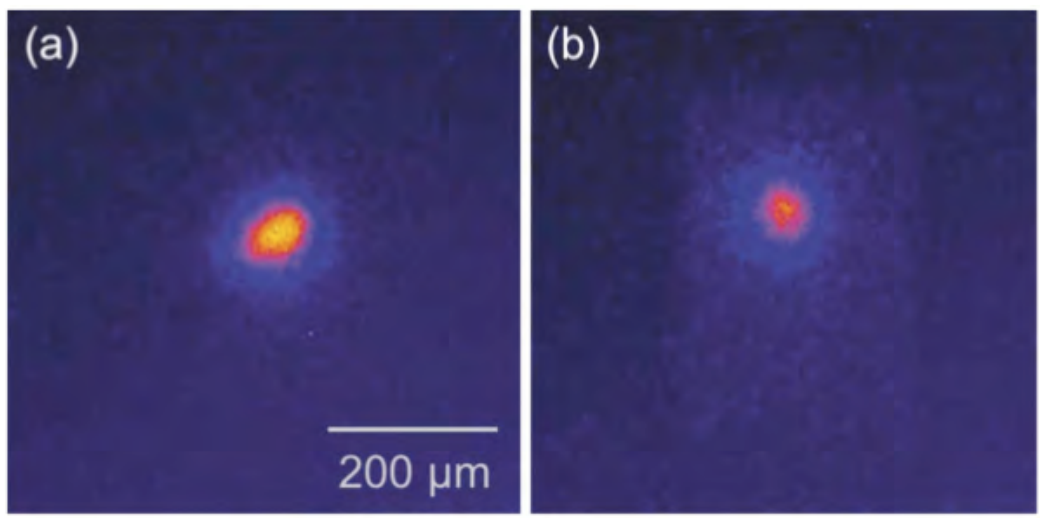

Figure 6.4: $\mathrm{K} \alpha$ images of targets irradiated by an essentially prepulse-free $2 \omega_{0}, 0.4$ ps laser pulse at best focus, $1 \times 10^{17} \mathrm{~W} / \mathrm{cm}^{2}$ intensity. In (a) a $\mathrm{Cu}(20 \mu \mathrm{m}) / \mathrm{Al}$ $(20 \mu \mathrm{m})$ target is irradiated directly, while in $(\mathrm{b})$, an $\mathrm{Al}(10 \mu \mathrm{m}) / \mathrm{Cu}(20 \mu \mathrm{m}) /$ $\mathrm{Al}(20 \mu \mathrm{m})$ planar target is attached to a $30^{\circ}, 20 \mu \mathrm{m}$ wide tip cone. The laser was focused directly on the target plane. Both images use the same color scale. Note that the appearance of a slightly lower $K \alpha$ spot signal in part (b) is due to the presence of the additional $10 \mu \mathrm{m}$ thick Al layer. Figure taken from Baton et al., Physics of Plasmas (2008)[96].

reproduce the essential features of the experiment, in particular, the shift in the peak emission back along the cone and the increased volume of emission with increased prepulse. The origin of these features can be understood by considering the laser interaction with the preformed plasma. In the $7.5 \mathrm{~mJ}$ prepulse case, filamentation and self-focusing begin occurring approximately $60 \mu \mathrm{m}$ from the cone tip, but one main filament is capable of boring a channel and reaches the tip to deposit most of its energy there. In the case of the $100 \mathrm{~mJ}$ prepulse, the onset of filamentation occurs approximately $120 \mu \mathrm{m}$ from the tip. Multiple filaments are created, halting propagation and accelerating electrons transversely directly into the side walls of the cone. Clearly, a large prepulse diverts electrons from propagating in the forward direction, and reduces the coupling to the tip of the cone.

These works seem to demonstrate the necessity to minimize prepulse levels as much as possible in order to maximize the coupling from laser to electron energy 

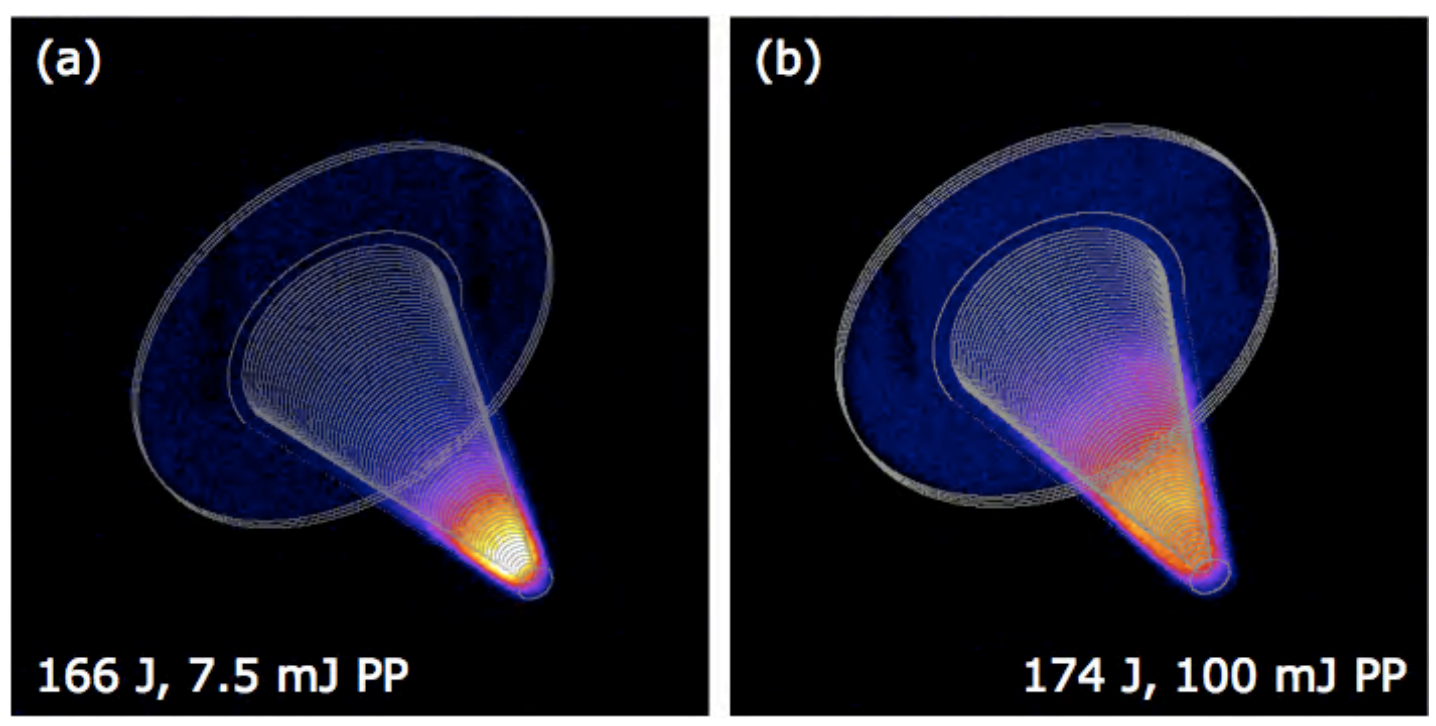

Figure 6.5: $\mathrm{Cu} \mathrm{K \alpha}$ emission from $\mathrm{Cu}$ cone targets irradiated with the Titan $150 \mathrm{~J}$, 0.7 ps laser pulse that is preceded by (a) $7.5 \mathrm{~mJ}$ and (b) $100 \mathrm{~mJ}$ energy, nanosecond duration prepulse.

beyond the tip of the cone. However, there is a very high engineering cost associated with improving the contrast levels of short-pulse lasers. Therefore, it is of utmost importance to actually pinpoint the tolerable level of prepulse. In other words, how much prepulse can we have and still have sufficient coupling to the fast ignition core, and how much prepulse is too much?

Thus far, only qualitative remarks on the effect of preformed plasma on the electron source have been reported. Here, we present the first quantitative scaling of coupling as a function of prepulse in an intense laser-cone interaction. Through the use of cone-wire targets, we measure the forward-going hot electron population at the cone tip that is of the most interest for fast ignition, and correlate their energy spectrum with the plasma in the cone. Electrons generated within the cone, and those forward-going at the cone tip would propagate into the wire, and then measurements of the absolute spatially-resolved $\mathrm{K} \alpha$ emission along the wire could be related to the original electron source. By varying the level of a controlled prepulse into the cone-wire target, and then measuring the coupling into the wire, a scaling of the effects of prepulse could be obtained.

As similar to the nail-wire experiment described in Chapter 5 , the wire provided 

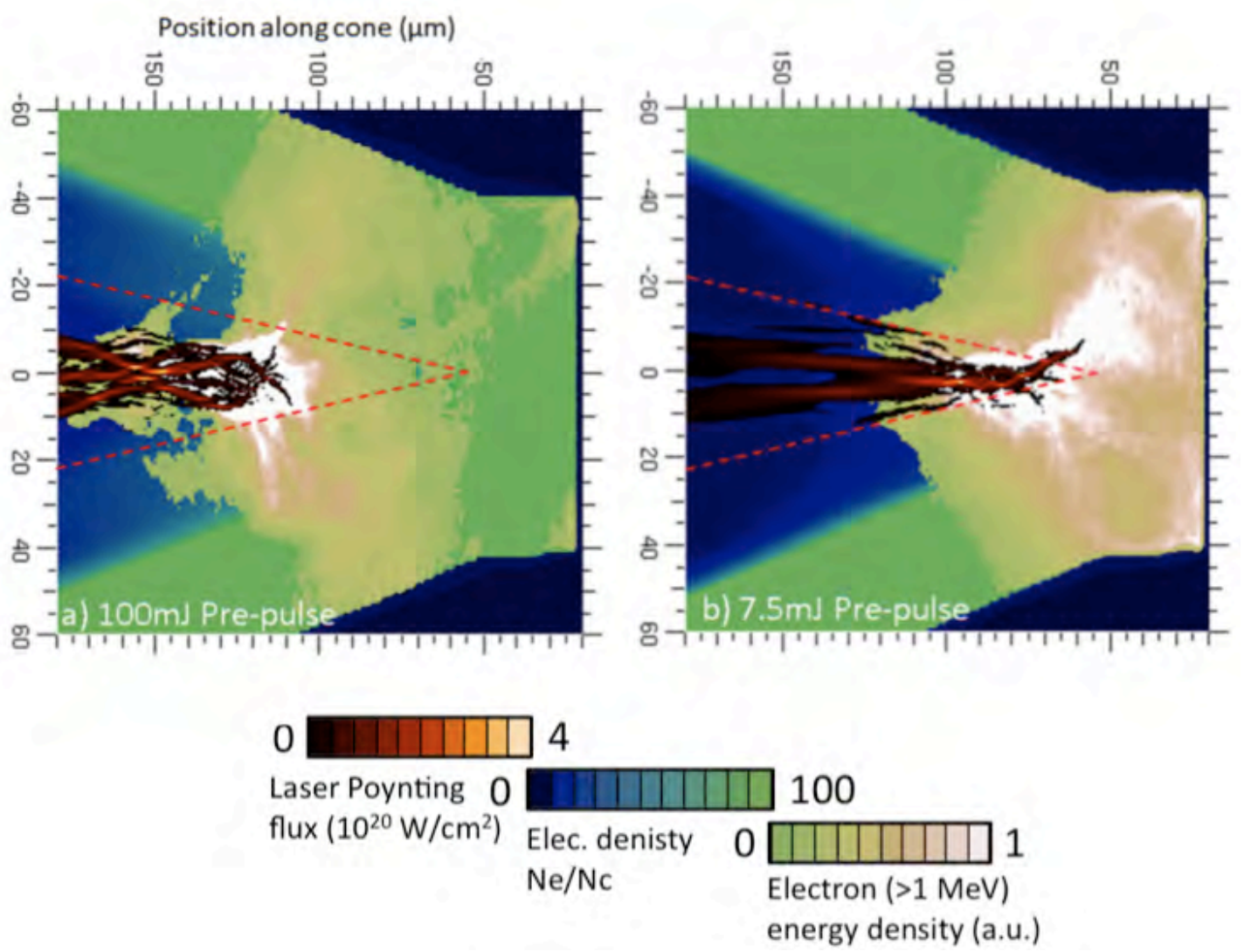

Figure 6.6: PIC simulation results for cone targets irradiated with two different levels of prepulse: initial electron density [blue-green], laser Poynting flux at time of peak fluence [red], and energy density of above $1 \mathrm{MeV}$ electrons at end of 3 ps simulation run [green-white]. Dashed lines show the geometric focus of the $\mathrm{f} / 3$ laser beam. Figure taken from MacPhee et al., Physical Review Letters (2010)[97].

a mechanism to examine hot electron transport without the complexities of a diverging beam. The simple quasi-1-D geometry of the wire made it accessible to multiple diagnostics, and simplified the modeling effort required to analyze the experiment. Further, the wire provided an open path for the generated hot electrons to propagate down so they would not re-interact with the laser at the absorption boundary. However, whereas in the nail-wire target both the nail-head and the wire were composed on a single material, the cone material was selected to be of a distinct material from the wire so that fluorescence from each portion of the target could be differentiated. 
While the aim was to perform a systematic study of the effect of prepulse on coupling into forward-going electrons in a cone geometry, we also endeavored to develop a robust set of modeling tools to tackle this type of analysis. Naturally, we would build on our knowledge of analysis of $\mathrm{K} \alpha$ fluorescence from electron propagation in narrow wire geometries as done by King et al.[14] and Ma et al.[99], but improve our understanding by using more complete physics models. In the previously cited studies, and in fact, in most previous calculations of conversion efficiency based on $\mathrm{K} \alpha$ emission[28, 27, 29], a purely collisional electron-stopping model was used. However, Bell et al.[42] drew attention to the fact that in the case of a high current of relativistic electrons, the penetration depth of these electrons can also be limited by the inability of the background plasma to provide a balancing return current. The heating of the plasma, and the resistive electric and magnetic fields induced by the propagation of these electrons can greatly complicate the transport interpretation. Detailed studies by Davies[37] and Honrubia[100] showed that even at a relatively modest laser intensity of $10^{18} \mathrm{~W} / \mathrm{cm}^{2}$, field effects could alter the inferred slope temperature and conversion efficiencies by a factor of 2 - 3 .

An extensive theoretical and experimental investigation of prepulse effects on electron temperature and conversion efficiency in cone-wire targets is presented here, in which we have additionally included and evaluated the contribution of the self-generated $\vec{B}$ and $\vec{E}$ fields.

\subsection{Experimental Setup}

The experiment was performed at the Jupiter Laser Facility, on the Titan laser, of $1.054 \mu \mathrm{m}$ wavelength, $150 \pm 10 \mathrm{~J}$, focused to an $8 \mu \mathrm{m}$ full width half maximum (FWHM) focal spot in a $0.7 \pm 0.2$ ps pulselength. The inherent intrinsic prepulse of the laser was $8 \pm 3 \mathrm{~mJ}$ in $1.7 \mathrm{~ns}$ at $7.5 \times 10^{10} \mathrm{~W} / \mathrm{cm}^{2}$. Additionally, an auxiliary laser was injected collinear to the main beam to simulate larger prepulse levels. This auxiliary laser was also of $1 \omega_{0}$ wavelength, and had similar focal spot properties as the main beam. It was timed to have a pulse duration of $3 \mathrm{~ns}$ (to overlap the full duration of the intrinsic prepulse), with the end of the pulse 
coinciding with the arrival of the main pulse. The laser energy on target provided by this auxiliary beam ranged from $0-1 \mathrm{~J}$.

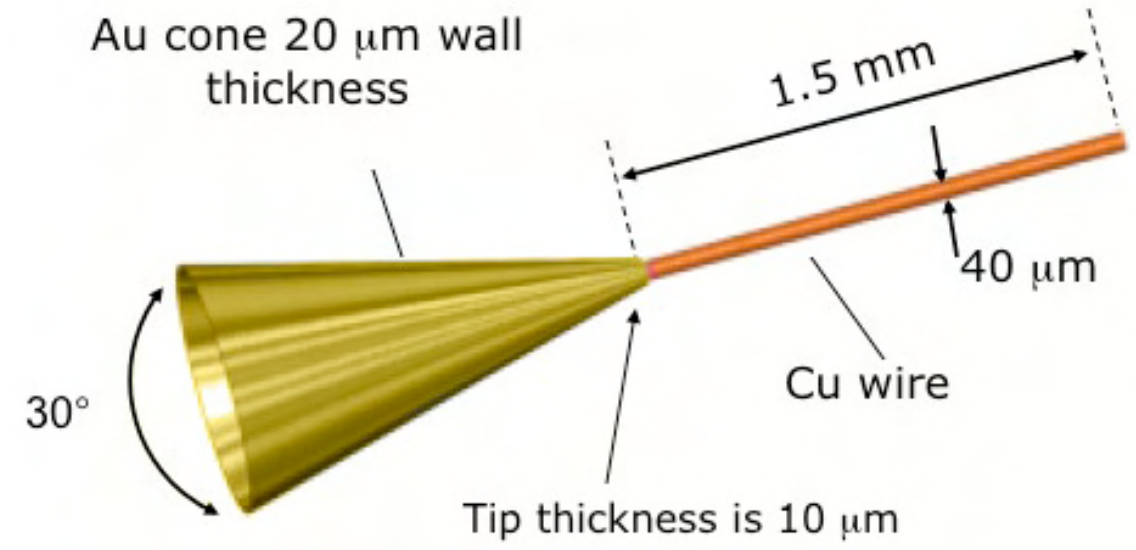

Figure 6.7: The cone-wire target. The $30^{\circ}$ full opening angle Au cone has $20 \mu \mathrm{m}$ thick walls, and an inner tip diameter of $30 \mu \mathrm{m}$, and thickness of $10 \mu \mathrm{m}$. A Cu wire of $40 \mu \mathrm{m}$ diameter, $1.5 \mathrm{~mm}$ length is glued to the end of the cone.

The targets irradiated were composed of a $40 \mu \mathrm{m}$ diameter $\mathrm{Cu}$ wire of $1.5 \mathrm{~mm}$ length, glued directly to the tip of a Au cone. The $40 \mu \mathrm{m}$ wire is representative of the nominal $40 \mu \mathrm{m}$ FI hot spot[8]. The Au cone had walls of $20 \mu \mathrm{m}$, a length of $1 \mathrm{~mm}$, an inner cone tip diameter of $30 \mu \mathrm{m}$, a tip thickness of $11 \mu \mathrm{m}$, and a full opening angle of $30^{\circ}$. A schematic of the cone-wire target is shown in Fig. 6.7. The $\mathrm{Au}$ cones were fabricated at the Rutherford Appleton Laboratory, and assembly of the wire to the cone tip using a UV hardened $\mathrm{CH}$ glue was done by General Atomics. Photographs of a typical Au cone-wire target is shown in Fig. ??. While best efforts were made to align the wire axis with the cone axis, typical offsets generally ranged from $5-10 \mu \mathrm{m}$. Each target was carefully metrologized and characterized to ensure dimensions were within specifications, the wire was mated well to the cone tip, and minimal residue from fabrication remained (i.e., the inside of the cone was clean, glue joining the wire with the cone tip was not excessive, etc.).

In the experiment, both the main laser pulse and the artificial prepulse beams were focused to the inner cone tip, straight down along the axis of the cone. To constrain the errors associated with misaligning the $\sim 8 \mu \mathrm{m}$ laser beam with the 30 

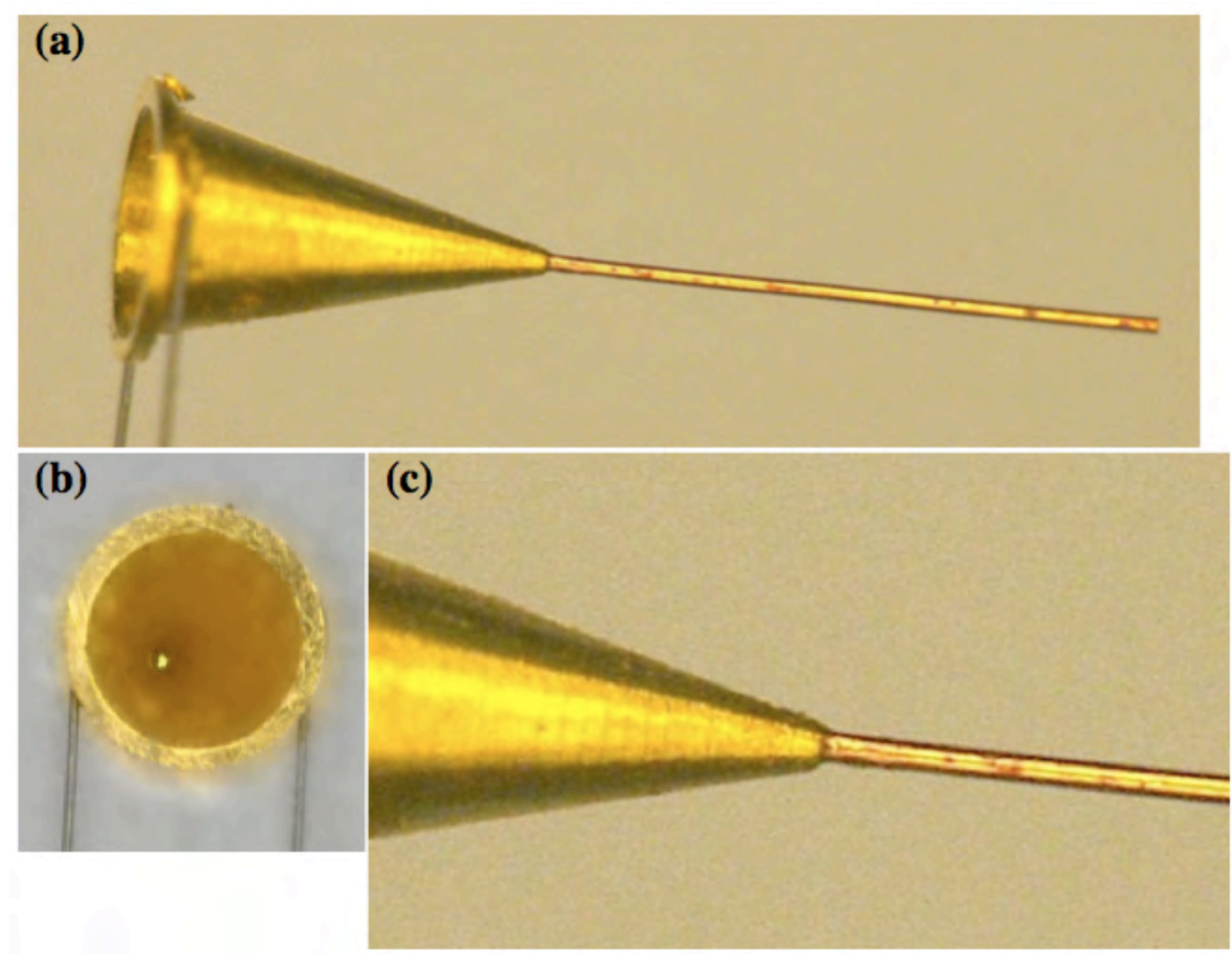

Figure 6.8: Images of the assembled cone-wire target, taken by General Atomics. Each target was individually metrologized to ensure dimensions were within specifications and the gluing of the wire to the cone tip was done well.

$\mu \mathrm{m}$ - diameter cone tip, extreme care was taken in the alignment process. First, the cone-wire target was aligned outside of the target chamber on the target alignment jig. The height, pitch, and yaw of the target were set to match the cylindrical axis of symmetry of the cone with the laser axis (i.e. the target was shot at normal incidence). The cone-wire target was then placed into the vacuum chamber, and front-illuminated (by reflecting white light off the final focusing parabola and into the cone) to determine the exact location of the cone tip before pumping down the chamber. Then, once the chamber was under vacuum, the CW (continuous wave) alignment beam (which propagates down the same beam path as the actual laser pulse, and is of the same wavelength, but is low enough intensity that it will not harm the solid target) was brought into the chamber. The position of 
the final focusing parabola was adjusted slightly before each shot such that the retroreflection of the $\mathrm{CW}$ alignment beam seemed to be focused onto the center of the inner cone tip, and the focal spot was optimized (i.e. was brightest and most symmetric).

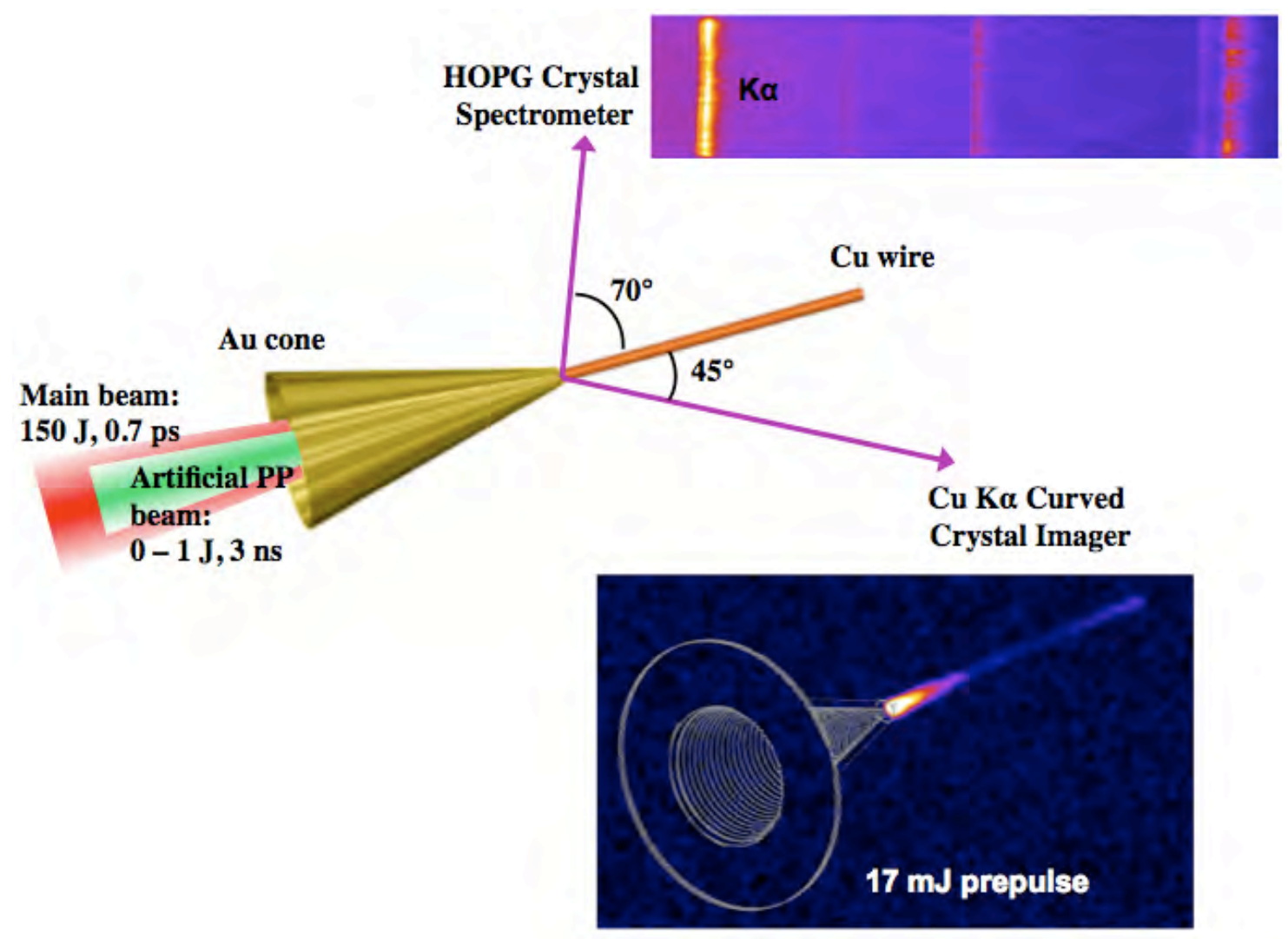

Figure 6.9: Experimental setup showing diagnostic view angles relative to the conewire target axis, and representative pieces of data from their respective diagnostic.

Fast electron-induced K-shell emission was used as the measurement for the number and distribution of the hot electrons. For every shot, two diagnostics viewed the x-ray emission from the $\mathrm{Cu}$ wire. The first was the spherical Braggreflecting crystal imager providing spatially resolved images of the $\mathrm{Cu} \mathrm{K} \alpha$ radiation. The $\mathrm{SiO}_{2}$ crystal detected radiation within a $\sim 6 \mathrm{eV}$ bandwidth around 8048 $\mathrm{eV}$, and relayed the image with a $7 \times$ magnification to a Princeton Instruments PI-SX1300 CCD camera. The second diagnostic was a Highly Oriented Pyrolithic Graphite (HOPG) spectrometer, consisting of a HOPG crystal with a random dis- 
tribution of scattering planes to allow Bragg reflection over a large spectral range. This provided an absolute yield of the total amount of $\mathrm{Cu} \mathrm{K} \alpha$ produced. The $\mathrm{K} \alpha$ imager viewed the target at $40^{\circ}$ below the horizontal axis, $20^{\circ}$ clockwise from the wire axis (for a compounded view angle of $\sim 45^{\circ}$, while the HOPG was located on the horizontal plane, at $70^{\circ}$ counterclockwise from the wire axis.

It is important to point out that there is a $10 \mu$ m-thick Au tip on the cone. This contains the full laser-matter interaction to occur within the Au cone. The $\mathrm{K} \alpha$ emission from the wire therefore originates from the electrons generated by the main short pulse, and which propagate through the tip of the cone into the wire. By capturing this emission, it is possible to infer the properties of electron number density and energy distribution of the initial electron source.

\subsection{Experimental Results}

A series of shots on the cone-wire targets were carried out, with several shots at the intrinsic prepulse level $(\sim 8 \mathrm{~mJ})$ to look at the shot-to-shot variability, and the remaining shots ranging in prepulse energy between $17 \mathrm{~mJ}$ and 1 J. Figure ?? shows the conversion efficiency into $\mathrm{K} \alpha$ (integrated $\mathrm{K} \alpha$ energy / laser energy) as a function of prepulse, using absolute yields as taken from the HOPG spectrometer. The reduction in coupling as the prepulse is increased into the cone is quite apparent, decreasing by approximately a factor of 8 from the lowest prepulse case to the maximum used in the experiment. The $21 \%$ error bars in the plot account for the random and systematic errors associated with the conversion of the $\mathrm{K} \alpha$ signal on the HOPG into absolute units (from a cross-calibration done against the signal recorded by a Single Photon Counting Camera[73], see Section 3.6.2.6 for more details on cross calibration of the $\mathrm{Cu} \mathrm{K \alpha}$ diagnostics). Quite interesting to note is the fluctuation in $\mathrm{K} \alpha$ conversion efficiency measured on the three repeated intrinsic prepulse shots. While the three shots were all taken on different days, they do represent very similar prepulse levels of 7, 8, and $10 \mathrm{~mJ}$, and were subject to practically equivalent laser conditions. This shot-to-shot variation in K $\alpha$ measurements has been seen in numerous experiments[29, 101], and can sometimes be 


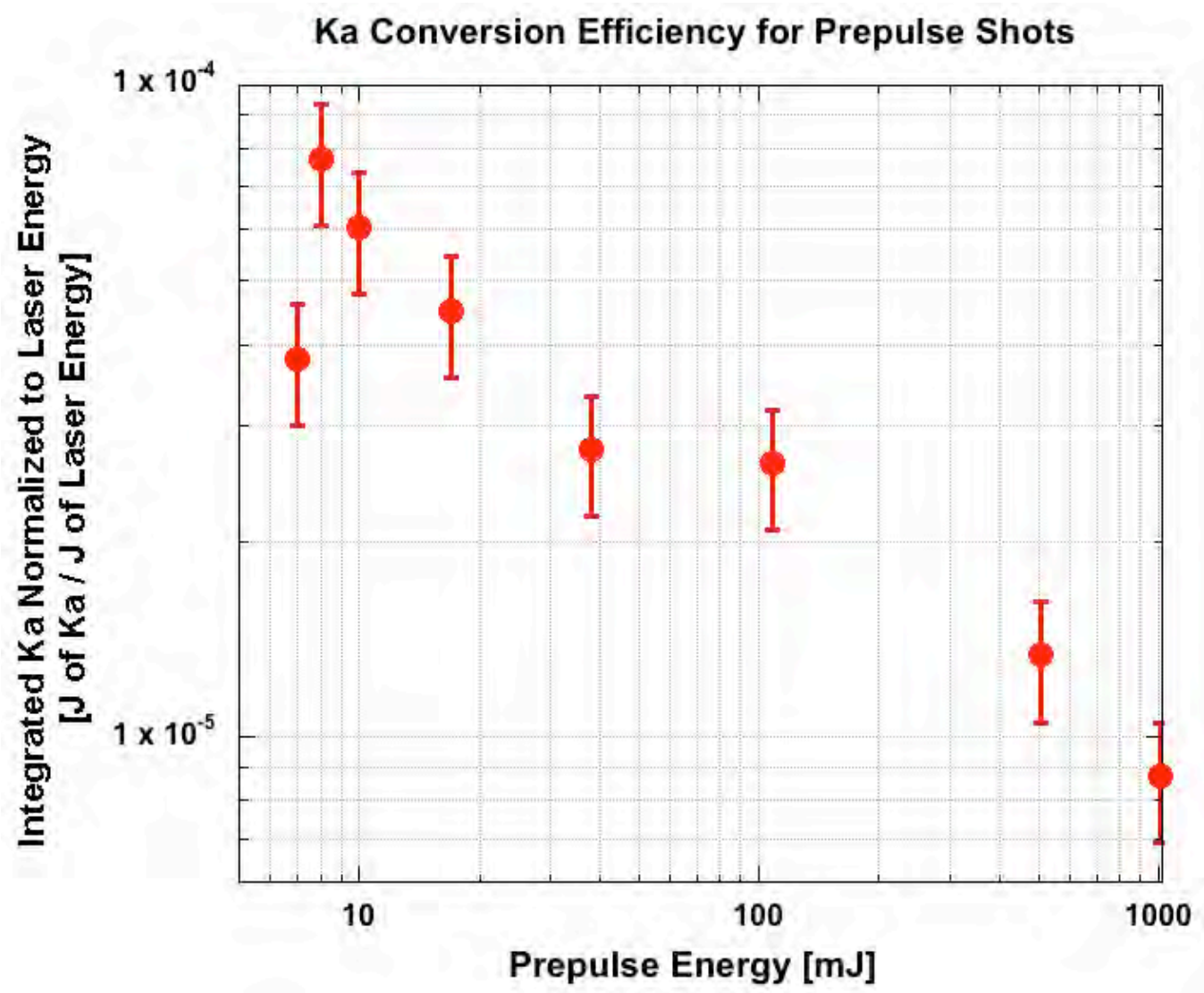

Figure 6.10: Integrated K $\alpha$ signal normalized to incident laser energy from conewire targets as a function of prepulse energy.

as great as a factor of two for identical repeated shots. The exact reasoning behind the variance is yet unknown, but is hypothesized to be due to some stochastic variation in the way laser energy is coupled into $\mathrm{K} \alpha$ energy, and may simply be an inherent characteristic in using $\mathrm{K} \alpha$ photon emission as a diagnostic.

Transversely integrated $\mathrm{K} \alpha$ lineouts along the wire axis (corrected for view angle and opacity) in absolute units of K $\alpha$ energy $[\mathrm{J} / \mu \mathrm{m}]$ for four shots of differing prepulse $(17,100,500$, and $1000 \mathrm{~mJ})$ are plotted out in Figure 6.11. Regardless of prepulse level, the axial profiles of $\mathrm{K} \alpha$ emission tend to follow similar trends: the $\mathrm{K} \alpha$ emission peaks at the beginning of the wire, then follows an approximately exponential drop-off for the first third of the wire (the first $500 \mu \mathrm{m}$ ), then levels off, before it begins rising again at the end of the wire. Approximately $60-70 \%$ of 


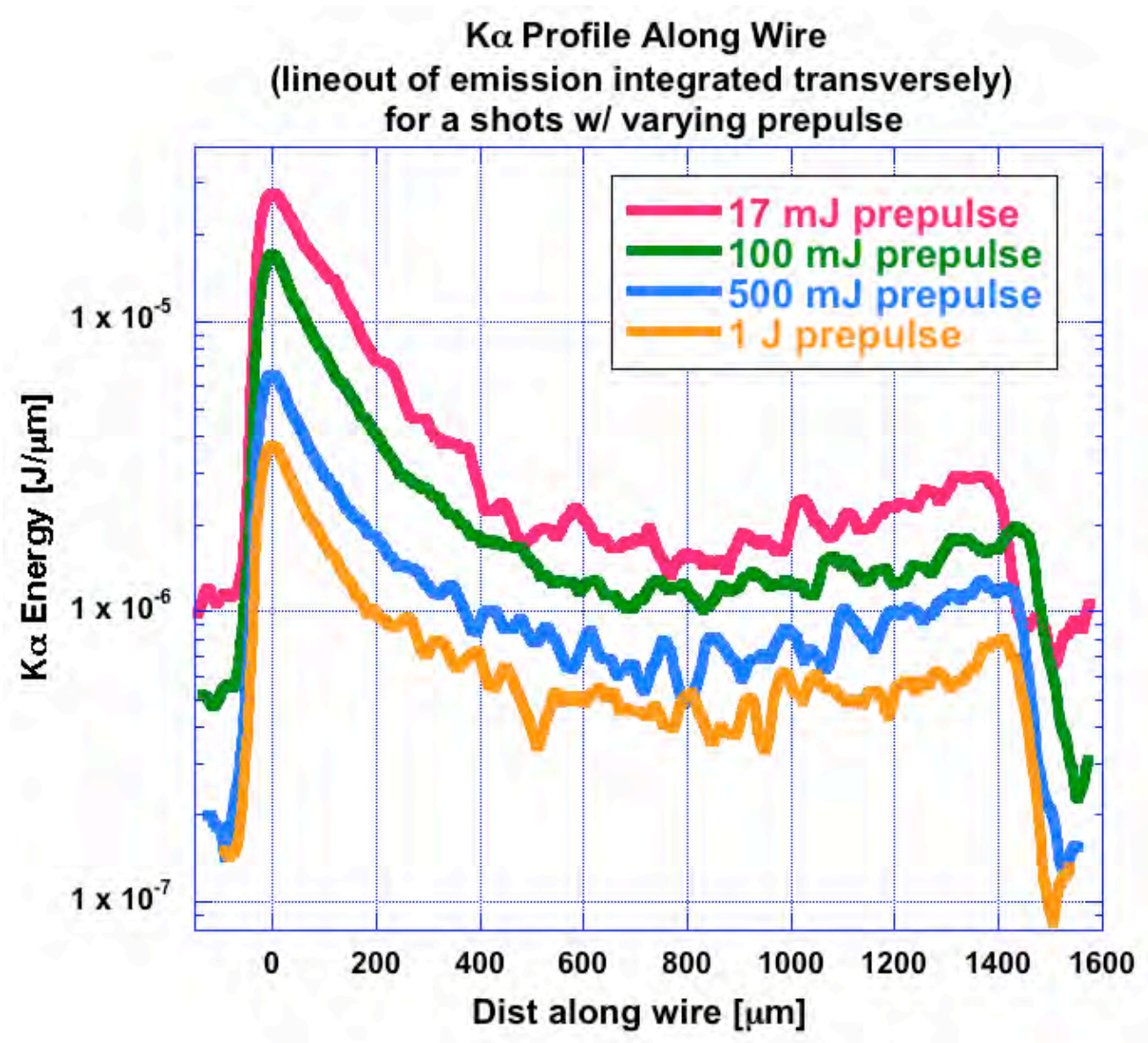

Figure 6.11: K $\alpha$ profiles taken along the wire (transversely integrated, corrected for view angle and opacity) for varying levels of prepulse.

the total K $\alpha$ signal lies within the first $500 \mu \mathrm{m}$ of the wire. The peak of the bump at the rear of the wire is a factor of 5 - 10 less than the $\mathrm{K} \alpha$ signal at the beginning of the wire, (factor of 10 in the intrinsic case, factor of 5 for the largest prepulse case), indicating that there is still a considerable amount of energy in hot electrons that manage to make it all the way down the wire and likely reflux (bounce at the wire termination) or get trapped at the end due to sheath fields.

In previous cases where the $\mathrm{K} \alpha$ imager diagnostic was utilized, it was found that the shift of the $\mathrm{K} \alpha$ line out of the bandwidth of the imager could result in significant loss of signal (see Sections 3.6.2.3 and 3.6.2.6 for more details). However, for this series of shots, this was determined to be a negligible effect. By directly 


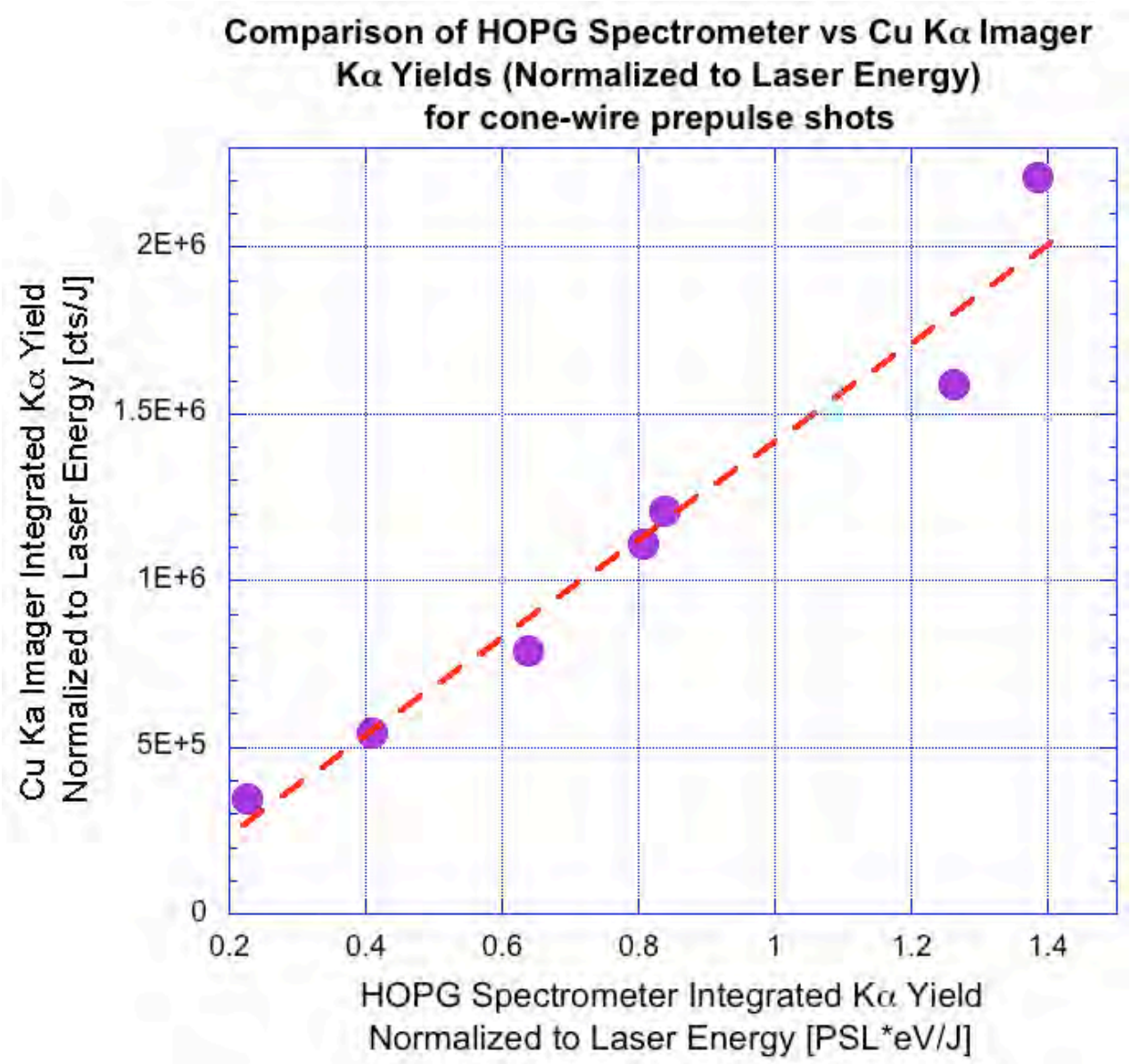

Figure 6.12: A plot of the integrated $\mathrm{K} \alpha$ yields as derived from the HOPG spectrometer versus the integrated $\mathrm{K} \alpha$ yields from the 2-D spatially resolved $\mathrm{Cu} \mathrm{K} \alpha$ Imager shows that the yields follow a fairly linear trend, indicating that the loss in signal on the imager due to shifting of the $\mathrm{K} \alpha$ line is minimal.

plotting the integrated $\mathrm{K} \alpha$ yields from the HOPG spectrometer (which captures the full $\mathrm{K} \alpha$ line including any shifting and broadening) against the integrated $\mathrm{K} \alpha$ signal from the $\mathrm{Cu} \mathrm{K} \alpha$ curved crystal imager for the same shot, over a series of data points where the $\mathrm{K} \alpha$ signal varies, a fairly accurate assessment can be made of this effect. At high $\mathrm{K} \alpha$ production levels, which generally corresponds to a large flux of hot electrons, and therefore heating that would induce shifting of the $\mathrm{K} \alpha$ line, we would expect to see the HOPG record more K $\alpha$ photons than the imager, and this disparity would increase for higher values of $\mathrm{K} \alpha$. If the relation between photons recorded by the HOPG and imager remain linear, however, as in the case 
of these shots (shown in Fig. 6.12), then the effect of shifting can be considered minimal. This will also be confirmed later by the low temperatures attained in the modeling.

\subsection{HYDRA Modeling of Preplasma Formation in Cone}

HYDRA, a 3-D multiphysics radiation hydrodynamics code[102], was used to model the hydrodynamic expansion of the inner cone walls due to irradiation by prepulse. The HYDRA code includes ion and electron thermal conduction, treated using flux-limited finite-element diffusion operators. Detailed radiation transport is calculated through Monte Carlo photonics or flux-limited multi-group diffusion. A number of tabulated and analytic EOS (equation of state) models are available, allowing for both LTE (local thermodynamic equilibrium) and non-LTE opacity models. Laser propagation is treated with a $3-\mathrm{D}$ ray trace algorithm to determine the spatial distribution of deposited laser energy, and includes ponderomotive effects. The Arbitrary Lagrange-Eulerian (ALE) method is used to stabilize shocks (by employing artificial viscosities), and to allow the grid to relax to therefore improve robustness of the mesh and stability of the code.

Simulations were undertaken in 2-D axially symmetric cylindrical geometry. Best efforts were made to match the experimental conditions, including modeling the actual target and using the true laser prepulse metrology. However, because the EOS of $\mathrm{Au}$ is of a somewhat classified nature, to avoid any possible conflicts, the cone material was modeled as a material at gold $(\mathrm{Z}=79)$ density, but with ytterbium $(\mathrm{Z}=70)$ EOS. Tests were run using commensurate material pairs to ensure that this was a valid approximation to make.

Runs were carried out corresponding to the same prepulse levels actually used in the experiment, and were run to $3 \mathrm{~ns}$ (the same amount of time the preplasma would have to evolve before the onset of the main pulse in the experiment). As can be seen in the electron density plots of Fig. 6.13, even in the case of the intrinsic Titan prepulse, it can be expected that a significant portion of the cone target is 

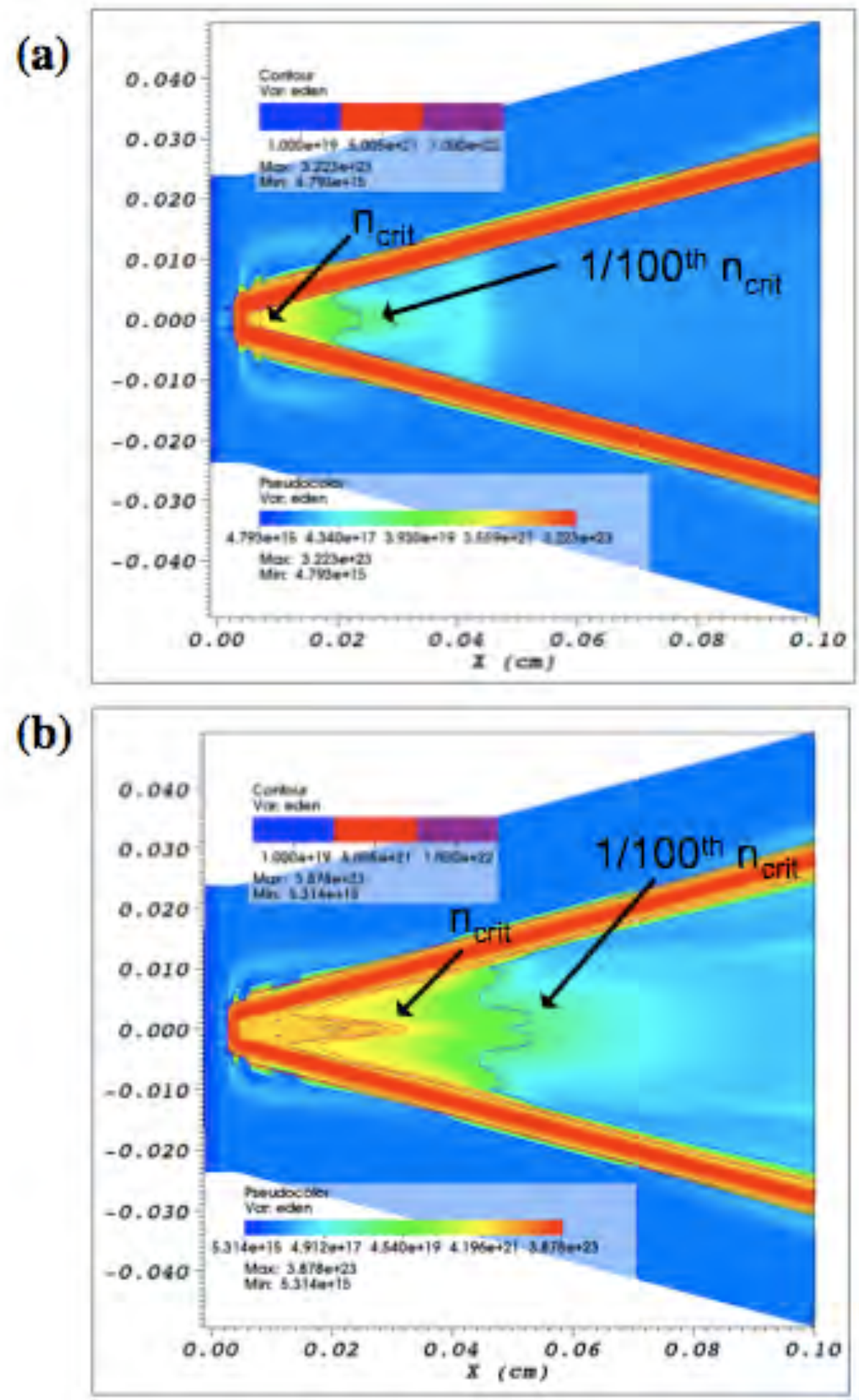

Figure 6.13: HYDRA simulation results of electron density at 3 ns in a Au cone with (a) $8 \mathrm{~mJ}$ of prepulse and (b) $1 \mathrm{~J}$ of prepulse in the nominal Titan ASE spot and pulselength. Electron density contours of $1 \times 10^{19}, 5 \times 10^{21}$, and $1 \times 10^{22}$ are marked. 
filled by the plasma (where $1 / 100^{t h} \mathrm{n}_{\text {crit }}$ can be roughly considered the extent of the underdense plasma). Even at the low prepulse levels, we begin to see evidence of an on-axis jet of plasma. As the laser is incident on the cone tip and walls (there is still a good amount of energy within the wings of the laser focal spot that hit the cone walls), and the plasma that is created expands out from the solid, there is a collision of the plasma on the axis of the cone. At larger prepulse levels, this on-axis jet becomes more and more pronounced. This is good confirmation of the confinement of plasma within the cone geometry suggested by Baton et al.[96].

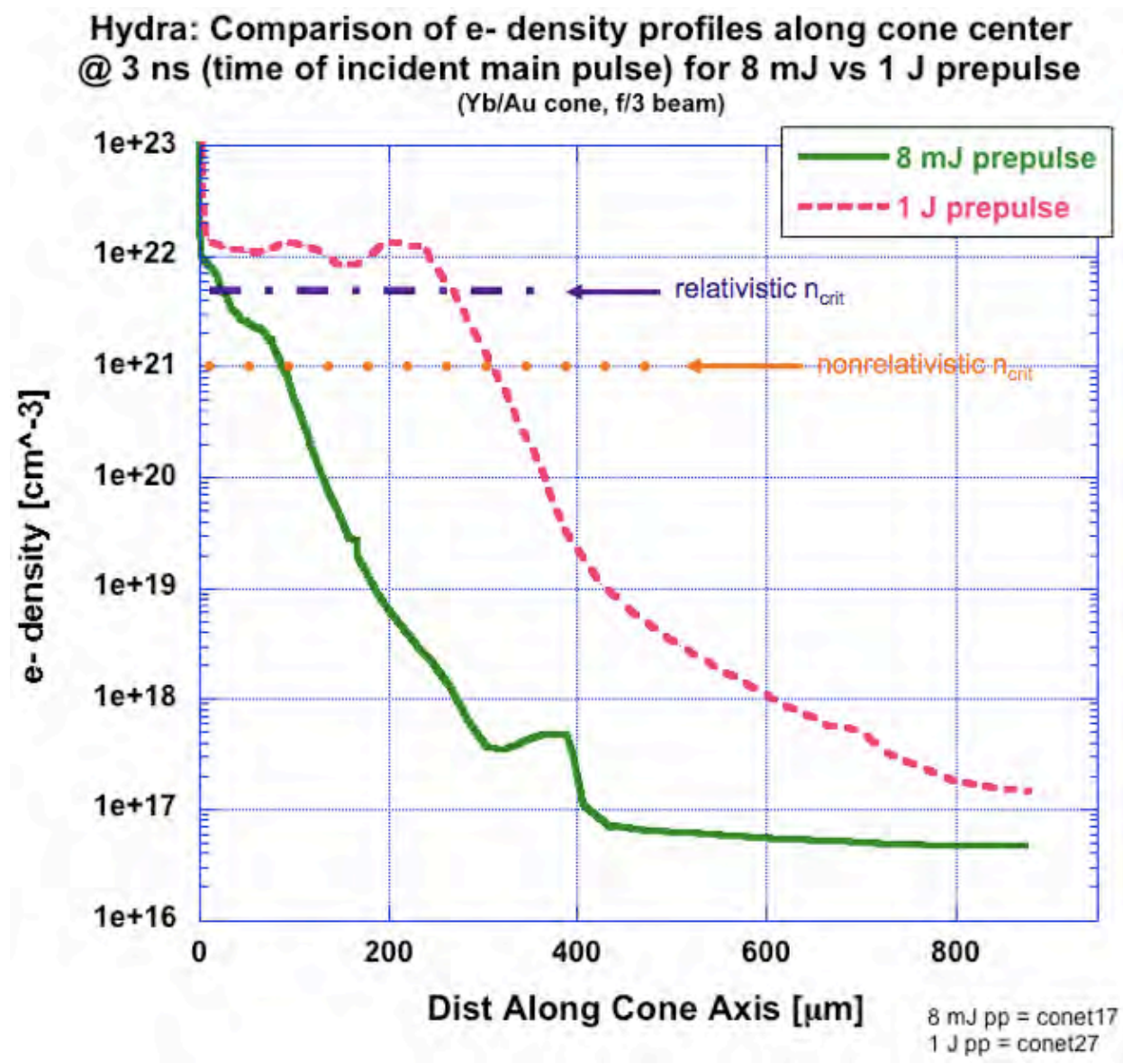

Figure 6.14: Electron density profiles along the axis of the cone. The relativistic and nonrelativistic critical density locations are marked.

A lineout taken along the axis of the cone with the expanded preplasma (Fig. 6.14 can give us an idea of what the main laser pulse would encounter upon entering 
the cone. At the $8 \mathrm{~mJ}$ level, the relativistic critical density, (defined as approximately $5 \times 10^{21} / \mathrm{cm}^{3}$ ) is at $\sim 25 \mu \mathrm{m}$ from the cone tip, and at $\sim 230 \mu \mathrm{m}$ for the $1 \mathrm{~J}$ of prepulse. The nonrelativistic critical density of $1 \times 10^{21} / \mathrm{cm}^{3}$ occurs at $\sim 85 \mu \mathrm{m}$ and $275 \mu \mathrm{m}$ for the 8 and $1000 \mathrm{~mJ}$ prepulses, respectively. Somewhere between the critical and noncritical densities (depending on the actual local intensity of the laser) is the approximate location of where the laser absorption would occur and hot electrons would be generated. Having the critical surface displaced so far back from the cone tip designates that the generated electrons will have over 200 $\mu \mathrm{m}$ farther to traverse in the case of the large prepulse over the small prepulse to even make it beyond the cone tip. Factoring in the fact that the generated electrons will also have some divergence (spread angle) in their trajectory, it becomes understandable why the coupling of electrons into the wire portion of the target systematically decreases as the magnitude of the prepulse increases.

\subsection{Inferring the Generated Hot Electron Distri- bution and Energy with Zuma}

To model the electron transport in the wire, the 3-D hybrid transport code Zuma was used. Zuma is based on the Davies[37]/Honrubia[100] hybrid models in which collisional Monte Carlo is combined with field generation. The background plasma is simulated as a resistive fluid, while the hot electrons are described kinetically with a Fokker-Planck equation. The transport of these fast electrons are modeled by taking into account both collisional and collective effects. Electrons are injected into the simulation box, then are slowed by collisions with the background and scatter off both the background ions and electrons. This is modeled via the drag and scattering formulas reported by Atzeni, Shiavi, and Davies[40]. For electric and magnetic field generation, the target is specified by its resistivity and heat capacity, and the fields are computed based on combining Ampere's law (without displacement current) with Ohm's law,

$$
\vec{E}=-\eta \vec{J}_{\text {hot electron }}+\frac{\eta}{\mu} \nabla \times \vec{B}
$$


and Faraday's law,

$$
\frac{\partial \vec{B}}{\partial t}=-\nabla \times \vec{E}
$$

The electric field is approximated as the gradient of the potential that arises to drive the return current. Resistivity is computed based on the Lee-More-Desjarles (LMD) expressions (the resistivity of $\mathrm{Cu}$ as a function of temperature used in Zuma is plotted in Fig. 6.15), and ionization is described by the Thomas-Fermi model. Thermal energy released via drag from the hot electrons goes into the background electrons (as determined by Thomas-Fermi) and ions. Equilibration between the background electrons and ions is assumed to be instantaneous. The K $\alpha$ emission was estimated using the $\mathrm{K}$ shell electron impact ionization cross section from the theoretical expressions of Hombourger[64].

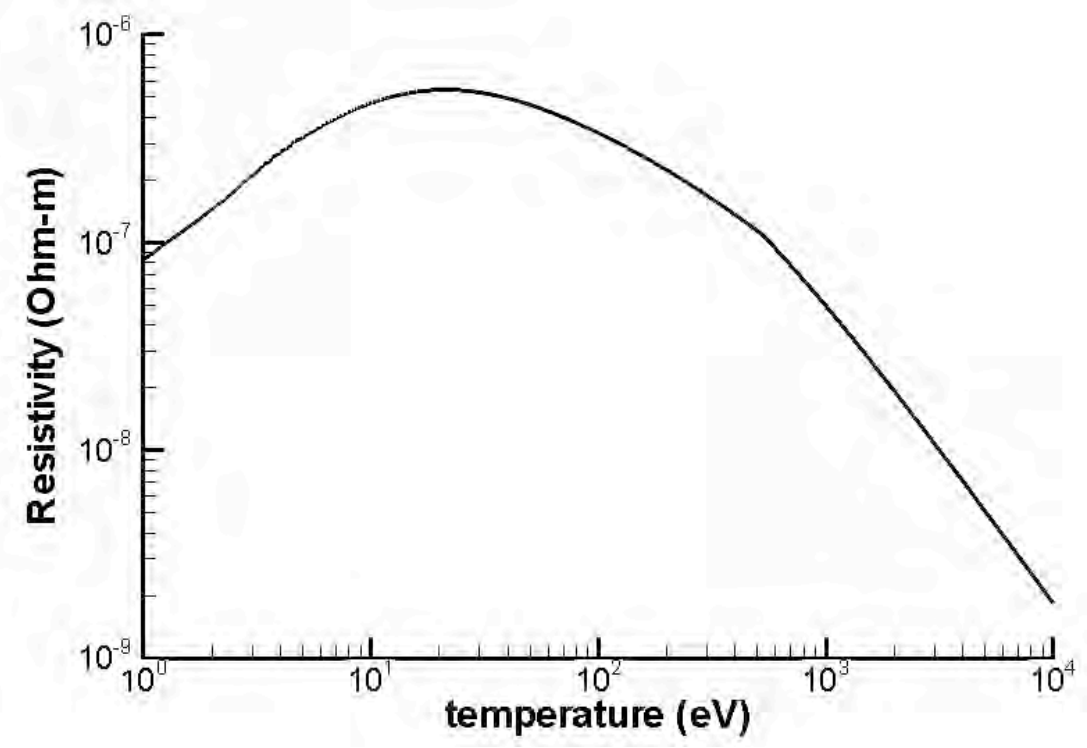

Figure 6.15: Resistivity of copper as a function of temperature, as calculated from the Lee-More-Desjarles (LMD) algorithm which is used in the Zuma code.

Simulations in Zuma could then help infer the energy deposition by hot electrons by generating $\mathrm{K} \alpha$ profiles along the wire. The wires were modeled at full scale: $40 \mu \mathrm{m}$ diameter, $1 \mathrm{~mm}$ long, Cu material, with an initial temperature of 0.1 $\mathrm{eV}$. To mimic the vacuum boundaries at the wire edges and back, those boundaries were made to be reflective, and as an initial assumption, the front boundary of the 
wire was also made reflective. In reality, electrons crossing the target/vacuum boundary would experience strong space charge effects which would turn the electrons back within the order of a Debye length, so the condition can be approximately represented by a reflecting boundary. In Zuma, electrons incident on this reflective boundary were specularly reflected back into the simulation box with the same energy.

The electrons were injected with a 3-D juttner-type relativistic Maxwellian distribution of the form,

$$
f(E)=\left(1+\frac{E}{m c^{2}}\right)\left[\left(1+\frac{E}{m c^{2}}\right)^{2}-1\right]^{1 / 2} \exp \left(-E / T_{h o t}\right)
$$

which has an energy spread which is illustrated in Fig. 6.16. The electrons had no

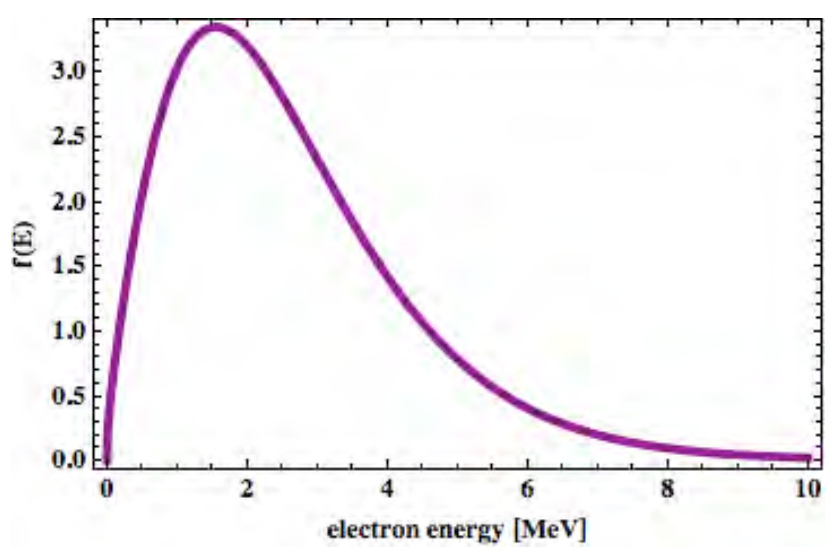

Figure 6.16: The distribution of electron energy for a $1 \mathrm{MeV}$, 3-D juttner-type relativistic Maxwellian distribution.

initial angular spread $\left(0^{\circ}\right.$ divergence angle), and were injected uniformly over the front face of the wire over the duration of the Titan pulse (0.7 ps gaussian temporal pulse). Because the full laser-plasma interaction and electron generation occurs within the Au cone, and Zuma is only modeling the transport of electrons that have advanced into the wire, the starting assumptions we have chosen are quite appropriate. Since the laser interface is removed, the injection of a population of electrons (rather than promoting them from the background) is valid. Hot electrons generated in the experiment with a wide divergence angle are likely to 
get lost in the plasma in the cone or in the cone walls, so electrons that do make it into the wire should have only a small component of transverse momentum, so it is reasonable to assume $0^{\circ}$ angular spread and a homogeneous injection over the wire front.

The runs were conducted in 3-D cartesian geometry, with cell sizes of $2 \mu \mathrm{m}$ in each $x-y-z$ coordinate direction. The temporal step size $(d t)$ was $2 \mathrm{fs}$, with a total of 7,000,000 particles injected, at a peak rate of 5,000 particles per time step. Simulation runs were typically carried out to 8 ps (slightly longer in the case of $>1 \mathrm{MeV}$ electron distributions to ensure that the electrons had sufficient time to deposit the majority of their energy). Two simulation input parameters were varied: hot electron temperature $\left(\mathrm{T}_{h o t}\right)$ and the laser-to-hot-electron conversion efficiency $(\eta$, in the form of total injected electron energy). A large parameter scan of simulations was completed with $\mathrm{T}_{\text {hot }}$ being varied from $250 \mathrm{keV}$ to $5 \mathrm{MeV}$, and the total injected electron energy ranging between $0.15 \mathrm{~J}-30 \mathrm{~J}$.

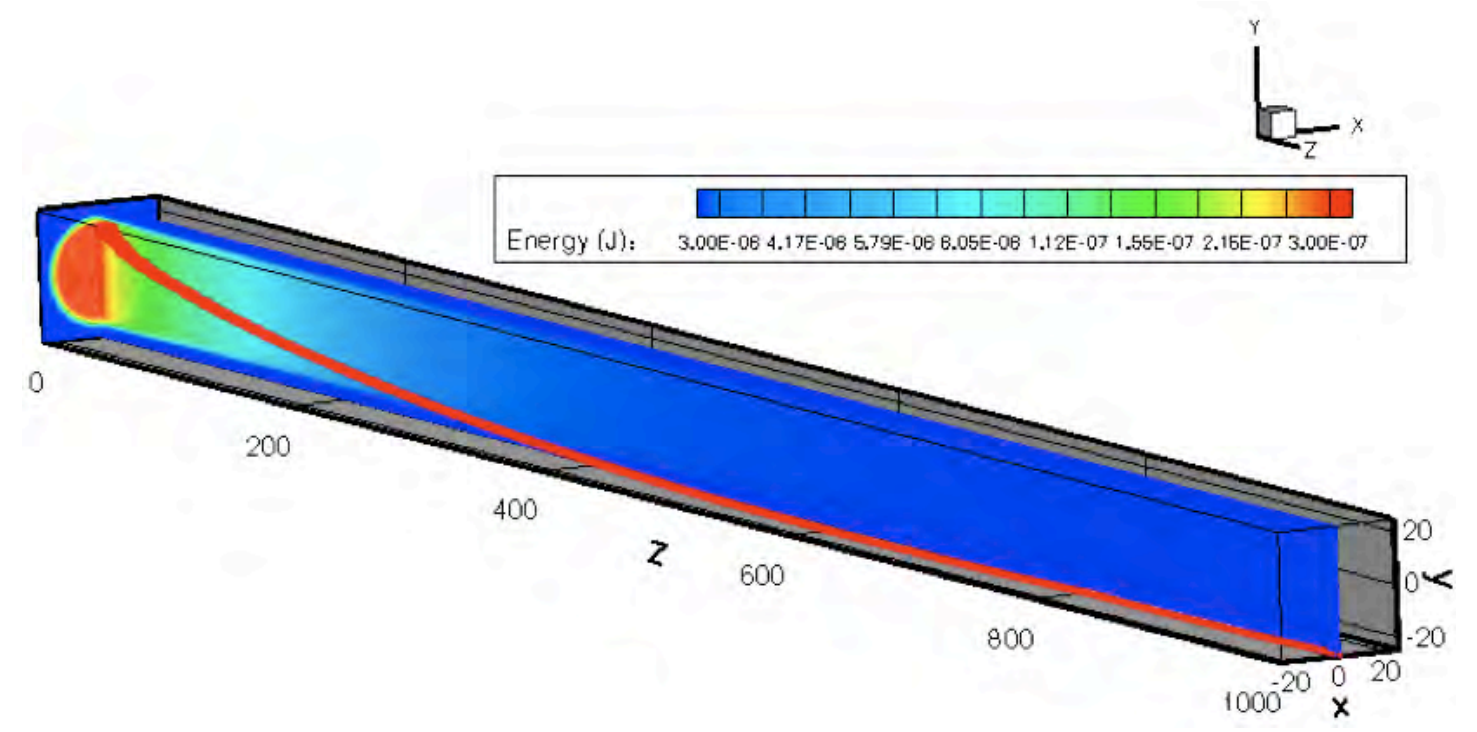

Figure 6.17: The K $\alpha$ generated along the wire as predicted by Zuma can be transversely integrated over time to give $\mathrm{K} \alpha$ profiles that can be directly compared against the experimental data. The color bar shows the integrated $\mathrm{K} \alpha$ up to 8 ps for a $1.5 \mathrm{~J}, 500 \mathrm{keV} \mathrm{T}_{\text {hot }}$ electron distribution injected into the wire.

Because the simulations were done at full target scale, and Zuma was able to 
predict the $\mathrm{K} \alpha$ generated in the wire at each time step, it was straightforward to postprocess the results. In the same way that the $\mathrm{K} \alpha$ profiles were derived from the experimental data, with the Zuma results, at each axial point along the wire, a transverse integration of $\mathrm{K} \alpha$ over the wire cross-section, over all time was completed. This gave profiles that could be directly matched to the experimental data, see Fig. 6.17. By finding the best fit Zuma-generated $\mathrm{K} \alpha$ profile for each experimental profile, the hot electron temperature and number density could be inferred.

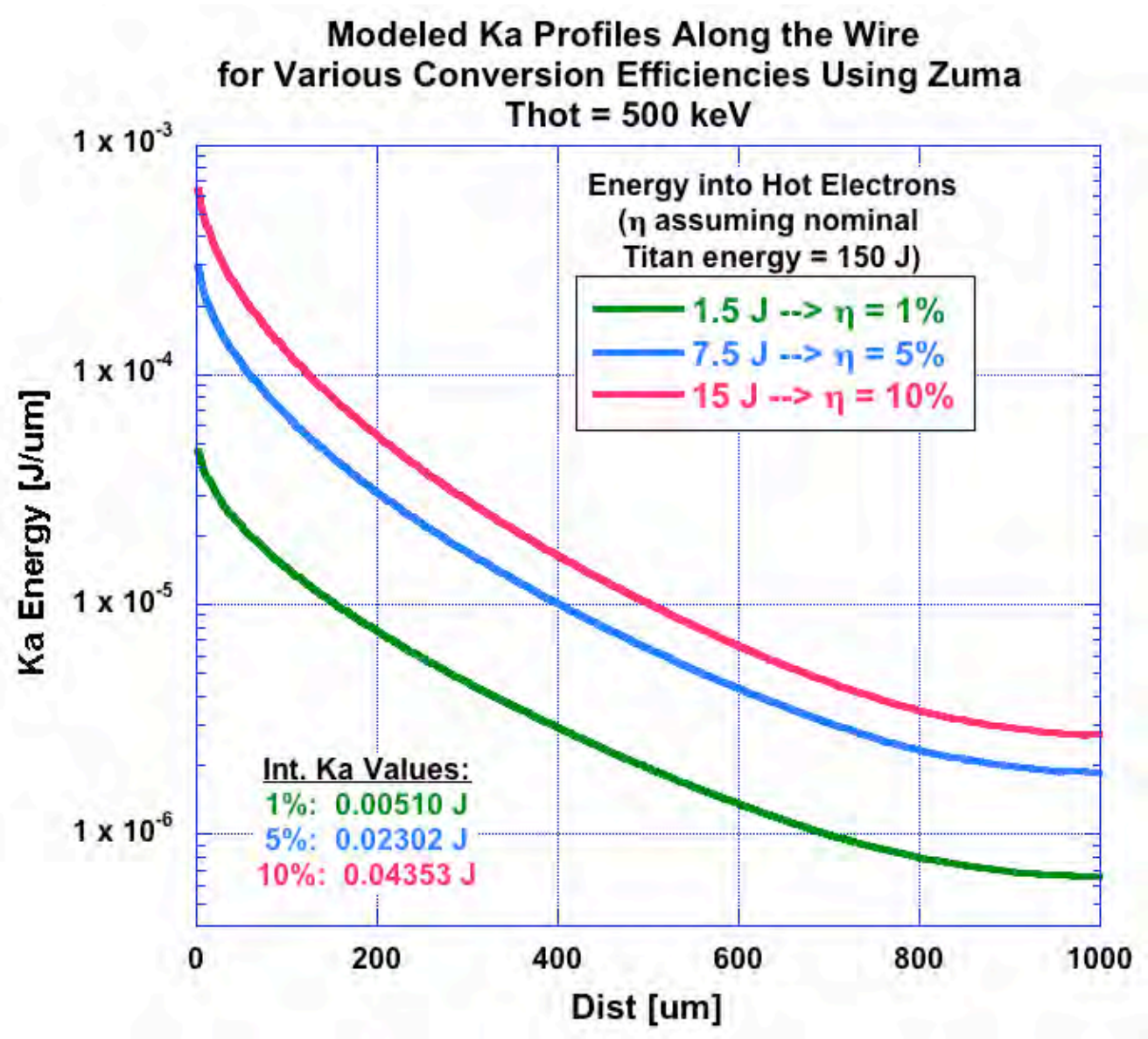

Figure 6.18: Zuma runs of $500 \mathrm{keV}$ and injected electron energies of 1.5, 7.5, and $15 \mathrm{~J}$ (corresponding to 1,5 , and $10 \%$ conversion of the Titan laser energy).

The relative ease and speed with which a Zuma run can be completed (a single run as described above, run in parallel on $8 \mathrm{CPUs}$ of $2.2 \mathrm{GHz}$ generally takes 3 - 
12 hours) allowed for a large parameter scan to be completed, as well as to explore the effects of changing various parameters of the simulation. Fig. 6.18 below shows three example simulation runs, all representing a $\mathrm{T}_{\text {hot }}$ of $500 \mathrm{keV}$, but with different amounts of injected electron energy $(1.5,7.5$, and $15 \mathrm{~J})$ corresponding to conversion efficiencies of 1, 5, and 10\% (assuming a laser of nominal Titan energy of $150 \mathrm{~J}$ ). The three profiles scale accordingly as expected, with more injected electron energy creating more $\mathrm{K} \alpha$ energy at every point along the wire.

Now, taking those same three profiles, and normalizing them to a single uniform point as in Fig. 6.19, we find that even though all three profiles represent the same slope temperature $\left(\mathrm{T}_{\text {hot }}=500 \mathrm{keV}\right)$, their initial drop off in K $\alpha$ energy is actually quite different. When more electron energy is injected, the slope is steeper (the $1 / e$ scalelength decreases), and as the injected energy is decreased, the slope begins to asymptotically approach the case of pure collisional stopping, as in having all the fields turned off (the orange, round dotted line). This shows that when the electron current is low, Ohmic stopping due to self-generated electric and magnetic fields does not play a big role, and the dominant stopping mechanism is collisional stoppping. At larger electron currents, resistive inhibition serves to lessen the depth of the electron penetration. This demonstrates that determining the $\mathrm{T}_{h o t}$ is not as straightforward as simply fitting the slope of the drop-off to some assumed electron distribution, as is often done in the analysis of electron transport experiments. The same measured 1/e drop-off length can mean very different temperatures depending on the overall electron number density, so in order to adequately fit our data, it is crucial to simultaneously match both parameters.

For each of the shots in our prepulse scan, the absolute transversely integrated $\mathrm{K} \alpha$ profiles from the $\mathrm{Cu}$ imager were compared to the Zuma models to determine the best fit profile, where both the total injected electron energy and the $\mathrm{T}_{\text {hot }}$ of those injected electrons were free parameters. Fig. 6.20 shows a shot taken with $17 \mathrm{~mJ}$ of prepulse, and the resulting $\mathrm{K} \alpha$ profile fit with three candidate Zumapredicted $\mathrm{K} \alpha$ profiles representing 250, 500, and $750 \mathrm{keV} \mathrm{T}_{h o t}$ 's, all with $1.5 \mathrm{~J}$ of hot electron energy. The Zuma profiles have been convolved with a gaussian function of $24 \mu \mathrm{m}$ FWHM to reconstruct the instrument function of the imager. 


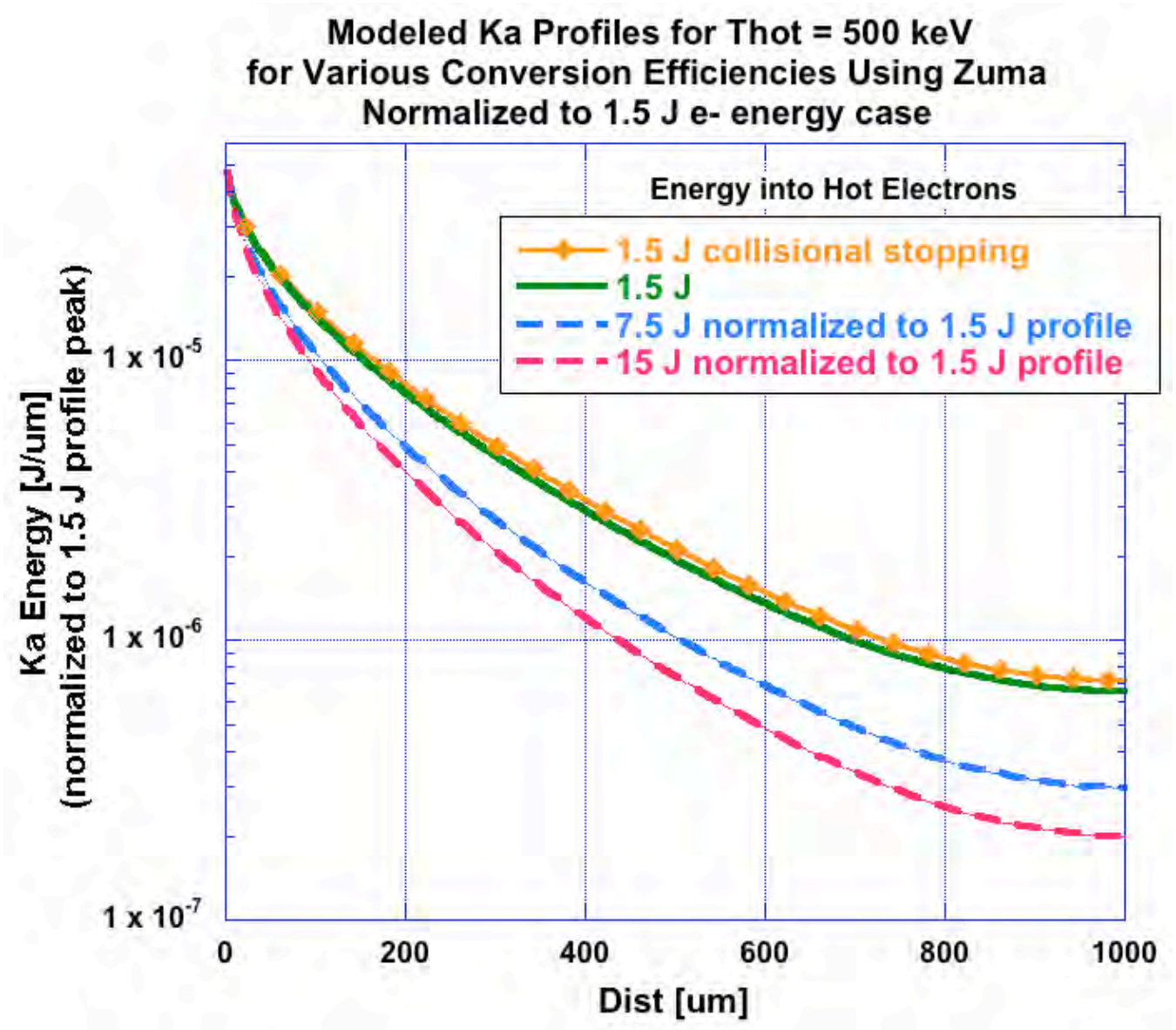

Figure 6.19: The K $\alpha$ profiles of Fig.6.18 normalized to a uniform point to display the difference in initial drop off, and the point at which we approach the purely collisional case.

In each fit, it is necessary to concurrently match the slope of drop-off, as well as the peak $\mathrm{K} \alpha$ value and the integrated $\mathrm{K} \alpha$ in the first $500 \mu \mathrm{m}$ of the profile. Only the first $500 \mu \mathrm{m}$ of each experimental profile is fitted in this analysis. Beyond that, the profile levels off or starts to rise again - evidence of either a higher temperature component in the electron distribution, and/or trapping of electrons by vacuum sheath fields. Repeated simulation trials in which extremely hot, single distributions of electrons $\left(1<\mathrm{T}_{h o t}<10 \mathrm{MeV}, 0.3<30 \mathrm{~J}\right)$ have been injected have thus far been unsuccessful in recreating any such rise at the wire end as is seen in the experimental data. A two-temperature parameterization may give closer results, but because it would have to be incredibly voluminous in scope in order to 
cover the many combinations of temperatures, and previous studies have found a large number on non-unique solutions when attempting 2-T fits[29], in this study, we restrict ourselves to only attempting an appropriate one-temperature fit. More advanced codes such as LSP or PSC will have to be utilized in order to adequately model the complex physics of the vacuum boundaries and fields.

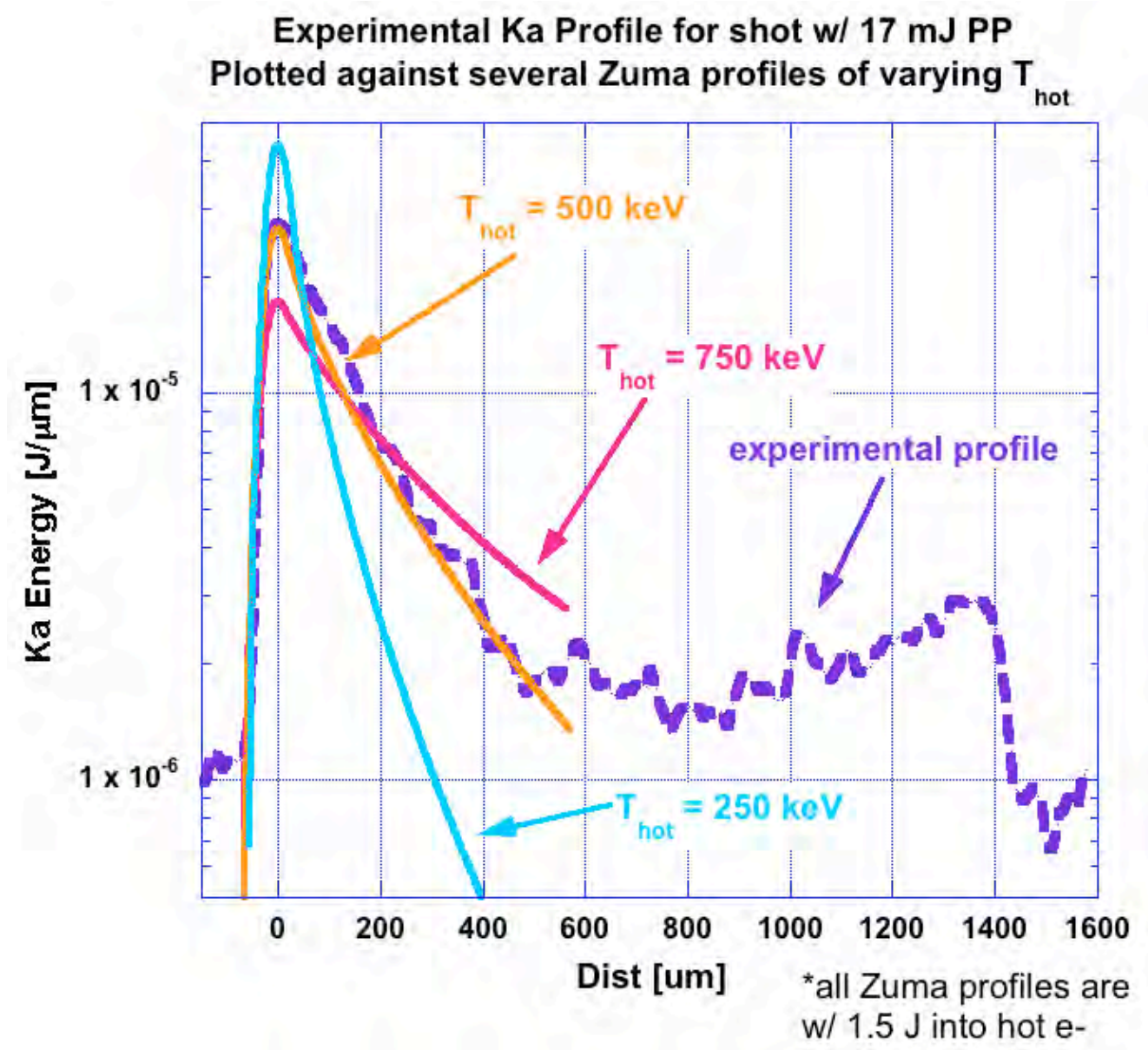

Figure 6.20: Experimental data for a shot with $17 \mathrm{~mJ}$ of prepulse (dotted line) fit with several Zuma simulations (solid lines).

Figure 6.20 therefore shows that the generated $\mathrm{K} \alpha$ profile from a Zuma run with $1.5 \mathrm{~J}$ of electron energy, and a $500 \mathrm{keV} \mathrm{T}_{\text {hot }}$ fits the experimental data of a shot with $17 \mathrm{~mJ}$ of prepulse quite well. With this process repeated for each piece of data, we can then convert the absolute $\mathrm{K} \alpha$ plot of Fig. 6.10 into a plot of conversion efficiency into the wire as a function of prepulse energy, as in Fig. 6.21. 


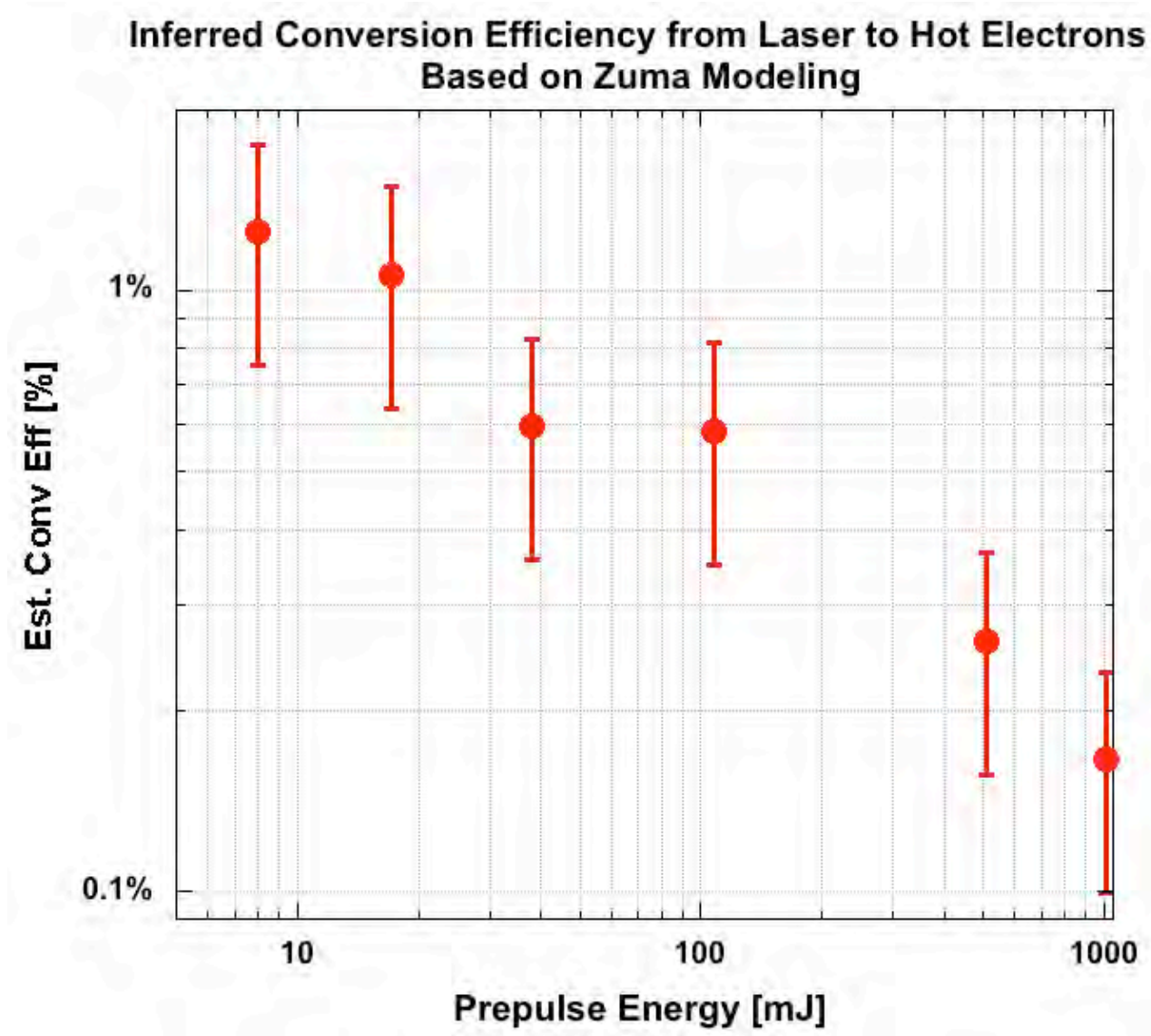

Figure 6.21: Estimated conversion efficiencies from laser energy to hot electron energy as inferred from the best fit Zuma calculation to the experimental data.

It was found that the conversion efficiency from laser-to-hot-electrons was highest for the case of the lowest prepulse $(8 \mathrm{~mJ})$, and corresponded to $\sim 1.1 \%$. The coupling then drops off logarithmically to a level of below $0.2 \%$ for $1000 \mathrm{~mJ}$ of prepulse. In this graph, the three intrinsic prepulse shots have been averaged, and their shot variation of $27 \%$ has been folded into the error bars (the other component being the error from the cross-calibration in determining the absolute value of $\mathrm{K} \alpha$ photons).

Similarly, from these Zuma simulations, we can also place a hot electron temperature on the distribution of electrons responsible for the $\mathrm{K} \alpha$ profiles seen for the various prepulse shots. These temperatures are marked on Fig. 6.22, and were not found to vary significantly across the prepulse levels. The temperatures tended to 


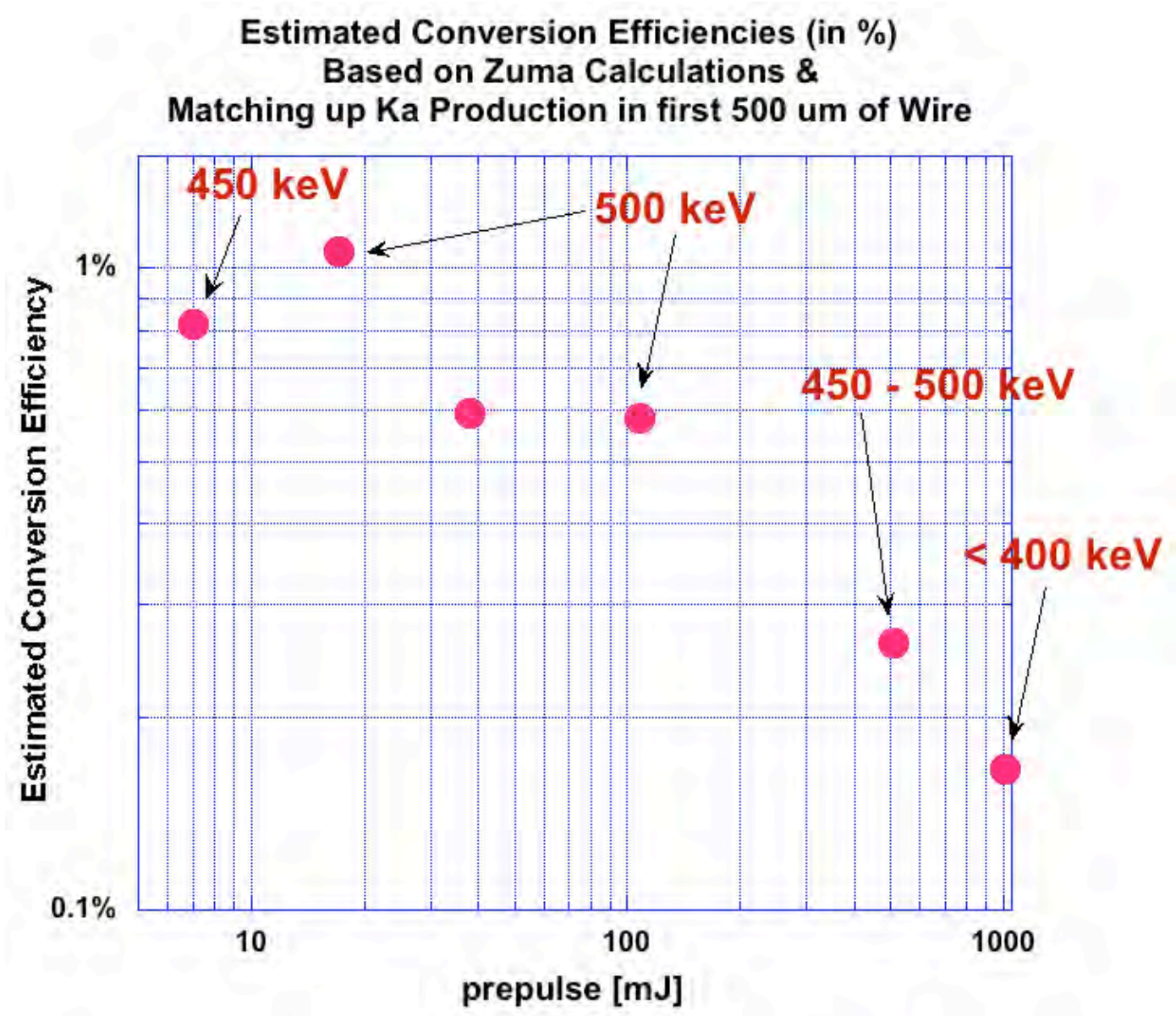

Figure 6.22: Estimated $\mathrm{T}_{\text {hot }}$ for each prepulse shot as inferred from the best fit Zuma calculation to the experimental data.

reside between 400 - $500 \mathrm{keV}$, with an error of $\pm 50 \mathrm{keV}$ due to fitting inaccuracies.

\subsection{Discussion}

By fitting the spatially resolved $\mathrm{K} \alpha$ profiles and the absolute $\mathrm{K} \alpha$ yields from cone-wire targets, an approximate scaling of laser to hot electron energy as a function of prepulse can be given,

$$
\eta_{\text {laser } \rightarrow \text { electrons }} \sim 1.6-0.5 \log \left(E_{P P}\right)
$$

where $E_{P P}$ is the prepulse energy for Titan laser conditions of $1 \mu \mathrm{m}$ light, $\mathrm{f} / 3$ focusing, and $2 \times 10^{20} \mathrm{~W} / \mathrm{cm}^{2}$ main pulse peak intensity. 
This decrease in coupling with increasing prepulse of course verifies what was observed by MacPhee et al.[97], and which is seen in the HYDRA simulations above: the larger the prepulse, the farther away $\mathrm{n}_{\text {crit }}$ and the electron source get pushed back from the cone tip. Hot electrons are therefore generated farther back in the cone, and must traverse a longer distance to reach the wire.

If we are to do a back-of-the-envelope geometric calculation of the fraction of electrons that would make it to the cone tip assuming a standard $40^{\circ}$ divergence angle (as empirically derived by Stephens et al.[75]), with electrons being generated from a point source on the cone axis at the location of critical density determined by HYDRA, we would find that approximately $3 \times$ more electrons would make it to the tip for the $8 \mathrm{~mJ}$ prepulse case than for $1000 \mathrm{~mJ}$. This is of course, a simplistic argument, but is still far less than the $8 \times$ decrease we observe in the experiment. Obviously, there must be some other mechanism other than the inherent angular spread of the electrons that is preventing them from reaching the cone tip.

The laser self-focusing and filamentation observed in the PSC calculations done by MacPhee et al.[97] are undoubtedly also at work in the prepulse scaling here. The larger the preplasma, the earlier the filamentation commences in the cone. In the process of the beam breaking up into smaller rays, the electrons are ejected with a transverse momentum that sends them propagating into the cone walls, stripping them of much of their forward-going directionality.

From our study, it would appear that the more we can improve the laser contrast and decrease the prepulse, the better the coupling would be. However, modeling studies by Kemp et al.[35] suggest that a small amount ( $>10 \mu \mathrm{m}$ scalelength) of preformed plasma is actually essential for high absorption of $\mathrm{MeV}$ electron energies. The preplasma is necessary to provide a smooth transition from a region of no plasma to overdense plasma in the bulk of the target. Without it, the laser would simply be reflected at the solid target interface. A preplasma allows the laser to interact with a density gradient.

At high laser intensities of $>10^{20} \mathrm{~W} / \mathrm{cm}^{2}$, the ponderomotive pressure of the laser can actually compress the plasma at greater-than-critical density, resulting in a steepening of the interface. In doing so, the preplasma scalelength can be 
shortened to a length such that the generated electrons no longer have the extent to accelerate to their full ponderomotive potential, resulting in a sub-MeV distribution. The electron temperatures inferred through the Zuma modeling of the cone-wires in this experiment were all found to be around $500 \mathrm{keV}$, across a large range of prepulse levels. This implies that the data did no reveal any evidence for ponderomotive steepening, as we would expect to see a higher $\mathrm{T}_{\text {hot }}$ at high prepulse (and longer preplasma scalelength) levels, and a lower $\mathrm{T}_{h o t}$ at lower prepulse levels. There can be two explanations for this: either (a) the preformed plasma is already so large that even in the small prepulse case the scalelength is already larger than the electron excursion length, or (b) the laser in this case was not of sufficient intensity for sufficient duration as to actually induce steepening.

It should be noted that the coupling efficiencies and electron temperatures derived from the Zuma simulations discussed above are lower limit estimates due to the assumption of total refluxing within the wire, i.e. once electrons are injected, they are trapped within the wire until they lose all their energy, boosting the amount of $\mathrm{K} \alpha$ they would appear to produce for a given electron distribution. Test cases were therefore run in Zuma with various parameters adjusted to determine what the effect of each would be on the inferred distribution.

In the original set of starting assumptions, the electrons are injected as a collimated beam ( $0^{\circ}$ divergence). As the divergence cone angle is increased, the peak $\mathrm{K} \alpha$ level at the beginning of the wire is raised, and the slope of drop-off in the $\mathrm{K} \alpha$ profile steepens. This can be explained by the fact that the larger the initial divergence angle, the larger the transverse component of the electron velocity. The path length of the electrons along the axis of the wire will therefore be abbreviated by a factor of $\sim \cos (\theta)$, meaning they will be stopped earlier in the wire, and produce more $\mathrm{K} \alpha$ near the front of the wire. As long as the boundary conditions remain reflecting, and the same number of electrons are injected into the wire, even in the case of an isotropic beam of electrons ( $180^{\circ}$ divergence), there is little effect on the absolute conversion efficiency into $\mathrm{K} \alpha$, but up to a $20 \%$ increase in the inferred $\mathrm{T}_{\text {hot }}$ due to the perceived steepening in the $\mathrm{K} \alpha$ profile.

Also in the original set of assumptions, reflecting boundaries were placed ev- 


\section{Effect of Fields, Divergence Angle, Reflecting Boundaries on various $500 \mathrm{keV}, 1.5 \mathrm{~J}$ runs}

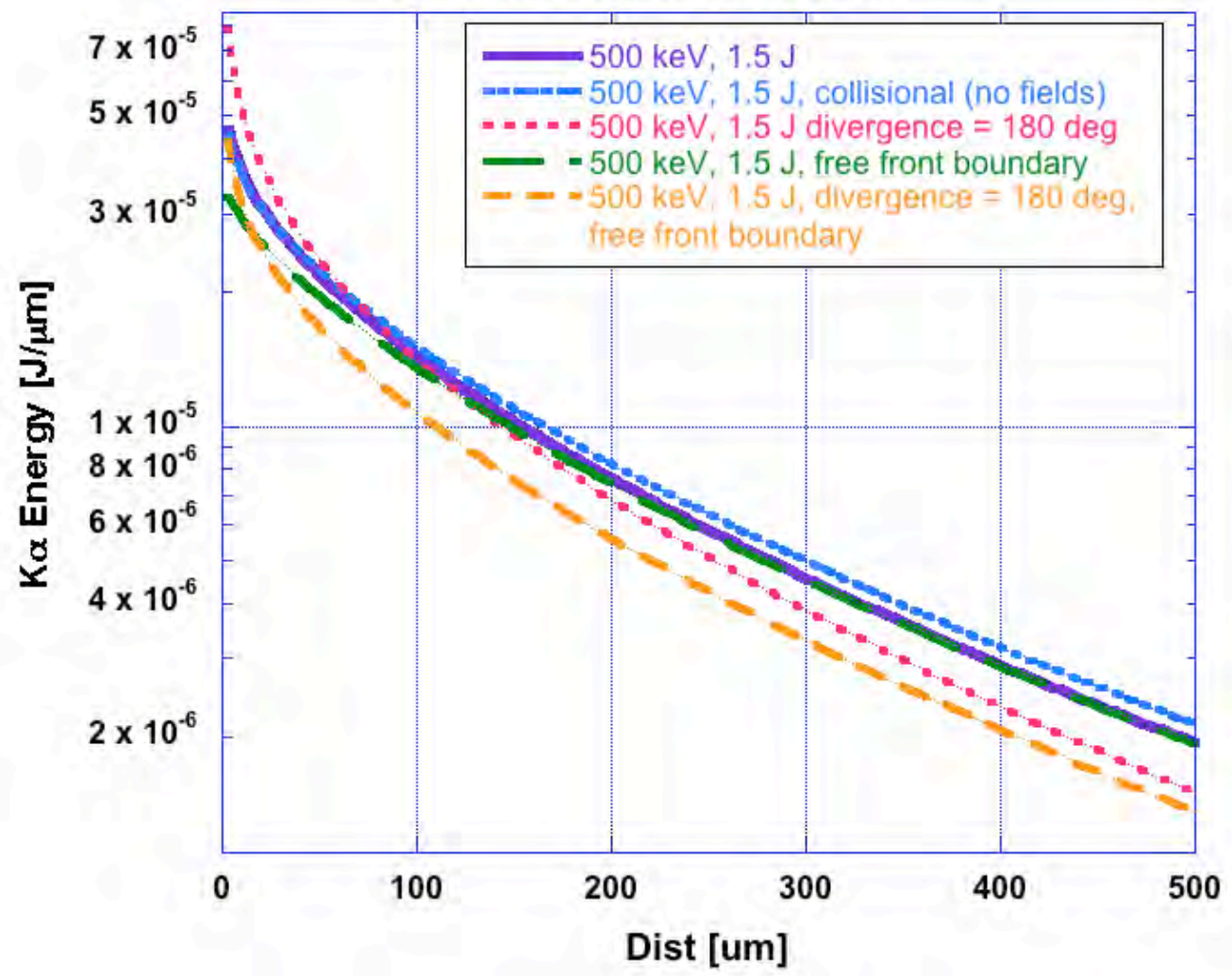

Figure 6.23: Predicted $\mathrm{K} \alpha$ profiles when divergence angle, reflecting boundaries are changed, etc.

erywhere around the wire geometry (wire front, back, and along the side edges). While the specularly reflecting boundaries at locations of a vacuum interface are an appropriate condition, it is reasonable that we would expect that at the front boundary where the wire is connected to the $\mathrm{Au}$ cone, that some electrons would be able to escape back up into the cone. Changing the front boundary to therefore be a free boundary, where electrons are free to escape, could boost the inferred conversion efficiency by up to $10 \%$ (for an isotropic electron distribution, less when assuming a collimated distribution).

In all cases, the energy lost to electric fields was determined to be $<5 \%$. In other words, for the range of electron fluxes and electron energy distributions that fit our set of cone-wire data, collisional stopping was the dominant stopping 
mechanism, and resistive stopping only played a minor role.

Fig. 6.23 shows the generated $\mathrm{K} \alpha$ profiles for various runs where electric and magnetic fields have been turned off, the divergence angle of the electrons is increased to $180^{\circ}$, and the front boundary condition is changed to a free one where electrons are free to escape. These can be compared to the profile of a $500 \mathrm{keV}, 1.5$ $\mathrm{J}$ electron distribution modeled using the original assumptions of $0^{\circ}$ divergence, all fields on, and fully reflecting boundaries everywhere along the wire.

One subtle, but final point to note is that in this analysis, we have only completed a one-temperature fit to the first $500 \mu \mathrm{m}$ of the $\mathrm{K} \alpha$ profile. To try to also fit the farther $2 / 3$ of the wire would require injecting two distinct electron distributions, with a total electron flux larger than used previously. This means that the first $500 \mu \mathrm{m}$ of the wire would experience a larger current density and consequently, increased resistive fields in that region. This would manifest in the form of a larger contribution from Ohmic stopping, and therefore would require a higher $T_{\text {hot }}$ necessary to fit the first portion of the wire (i.e. the cooler component of the fast electron distribution would be predicted to be a higher temperature). Therefore, it must be emphasized that the conversion efficiencies quoted here are applicable only to coupling from the laser into hot electrons in the forward-going direction that make it into the wire to deposit $K \alpha$ with a one-temperature $\sim 500$ ke $V$ distribution in the first $500 \mu \mathrm{m}$.

Those that are familiar with cone-wire and hot electron coupling efficiency experiments may ask why the conversion efficiencies measured here are approximately an order of magnitude lower than the ones quoted in the Physics of Plasmas paper by J. King et al.[14]. In that study, a cone-wire target (where the wire was $40 \mu \mathrm{m}$ thick $\mathrm{Cu}$, and the $30^{\circ}$ full-opening angle cone was $\mathrm{Al}$ with $10 \mu \mathrm{m}$ thick walls, a $5 \mu \mathrm{m}$ thick cone tip, and $30 \mu \mathrm{m}$ inner diameter), gave a conversion efficiency of $15 \%$ into $750 \mathrm{keV}$ hot electrons. A number of factors may have contributed to this much higher coupling efficiency being measured. First, the J. King experiment was performed on the RAL petawatt laser, at a higher energy of $345 \mathrm{~J}$, corresponding to an on-shot intensity of approximately $2 \times$ higher than what was achievable on Titan. The exact prepulse level on the RAL laser is unknown, although it is 
expected to be at least as great as the intrinsic prepulse level on Titan. Perhaps the higher laser intensity allowed for higher absorption or facilitated channeling through the underdense plasma.

Also, the cone in J. King's paper is aluminum, while the one used in this analysis is gold. The side walls of the $\mathrm{Al}$ cone were only $10 \mu \mathrm{m}$, while the $\mathrm{Au}$ cone used here had $20 \mu \mathrm{m}$ thick walls. It has been shown that coupling into the forward-going direction decreases as a function of cone side wall thickness[103]. The areal density of a $20 \mu \mathrm{m}$ thick Au cone wall is more than $14 \times$ greater than a $10 \mu \mathrm{m}$ thick Al wall, meaning we would expect about $4 \times$ less coupling, using a similar scaling as described by Akli et al.[103]

The Al cone tip was only half as thick as the Au cone tips $(5 \mu \mathrm{m}$ versus $11 \mu \mathrm{m}$.) The electron stopping within the thicker $\mathrm{Au}$ would of course be greater than for the thinner $\mathrm{Al}$. In addition, the scattering cross section for fast electrons scattering off plasma ions and electrons scales as $Z^{2}$, so we would expect much more scattering within the Au tip than the Al tip, perhaps redirecting electrons into a larger solid angle and reducing the number and energy of electrons making it into the wire. Zuma simulations show that approximately $12 \%$ of the coupled electron energy of a $500 \mathrm{keV} \mathrm{T}_{\text {hot }}$ electron distribution would be lost to an $11 \mu \mathrm{m}$ thick Au cone tip, while only $\sim 4 \%$ would be lost to a $5 \mu \mathrm{m} \mathrm{Al} \mathrm{cone} \mathrm{tip.}$

One other difference is that while the techniques are similar in both analyses, the Zuma code utilized here is more advanced and complete than the 1-D model used in the J. King et al. paper. Combined with a more precise cross-calibration to obtain absolute $\mathrm{K} \alpha$ yields, the errors in this analysis are significantly smaller than those in the J. King analysis (40\% in the inferred conversion efficiency and $<15 \%$ in the $\mathrm{T}_{\text {hot }}$, versus $78 \%$ in the conversion efficiency and $63 \%$ in the $\mathrm{T}_{h o t}$ ). Taking into account all these factors, the numbers derived in the analysis here are not in contradiction with those cited by King et al., and perhaps serve as a refinement on the previous analysis. Efforts continue to quantify the differences in coupling due to laser intensities, prepulse levels, cone materials, cone geometries, etc.

For the prepulse scan here, we were limited to the minimum prepulse level of $8 \mathrm{~mJ}$ 
achievable on the Titan laser. From the HYDRA simulations, we know that even with $8 \mathrm{~mJ}$, a considerable preformed plasma is created inside the cone, with the critical density pushed out to $\sim 25 \mu \mathrm{m}$ from the cone tip. It is a logical question, then, to ask what the coupling would look like were there to be no prepulse at all?

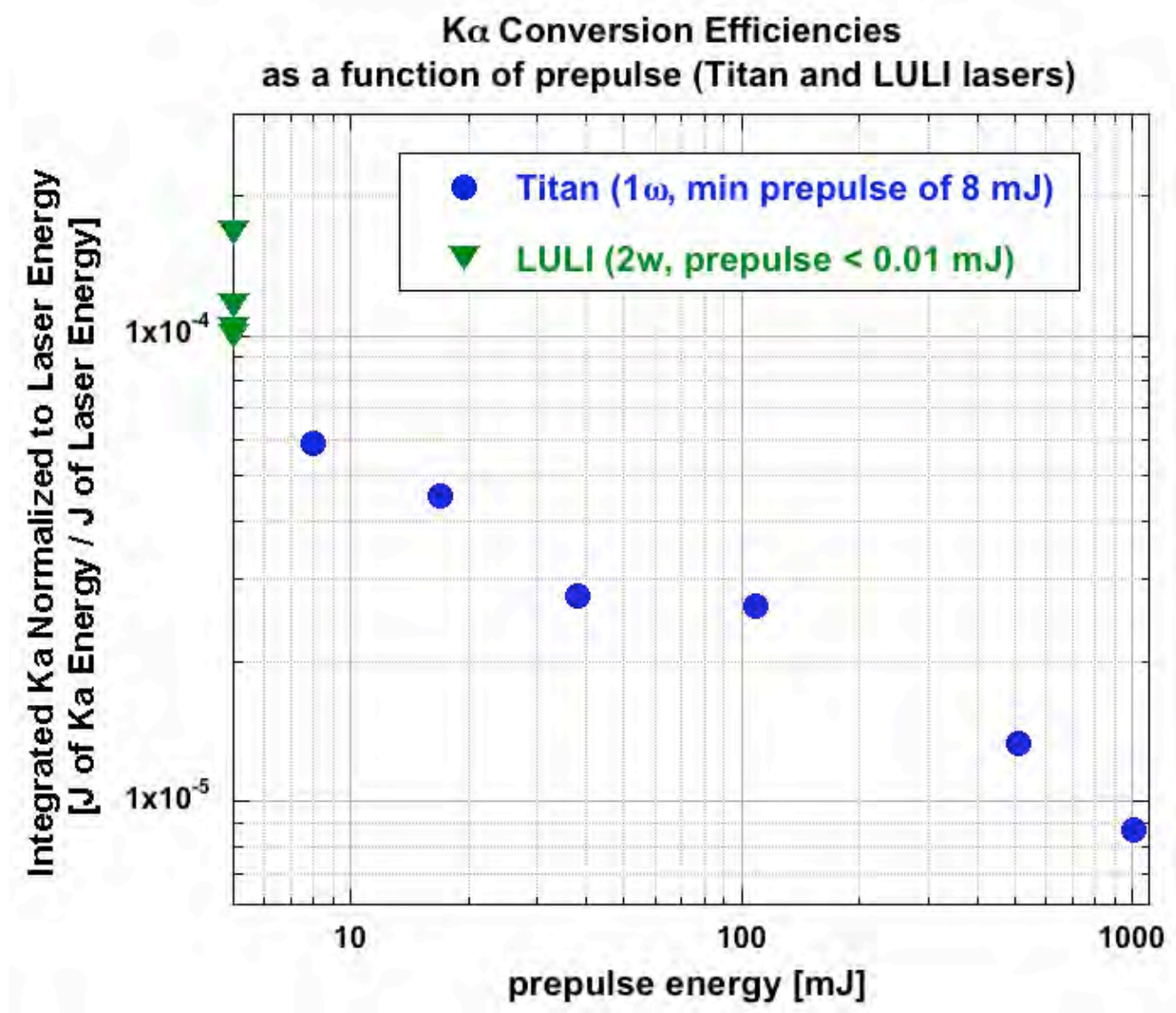

Figure 6.24: Identical cone-wire targets shot using the $2 \omega$ laser at the Laboratoire pour l'Utilisation des Lasers Intenses (LULI) showed coupling into the wire of approximately a factor of two higher than seen on Titan. This is likely due to the nearly zero prepulse at LULI.

To answer the question, the identical cone-wire targets and the same set of diagnostic instruments was taken to the Laboratoire pour l'Utilisation des Lasers Intenses (LULI) laser in Palaiseau, France. LULI is an ultra-intense $2 \omega_{0}$ laser of 25 J, $850 \mathrm{fs}$, and a $4 \mu \mathrm{m}$ FWHM focal spot. Through the process of frequency doubling the laser pulse, the original $1 \omega_{0}$ pulse must be sent through a nonlinear doubling 
crystal and is reflected off three dichoric mirrors which brings the intensity contrast level to better than $10^{10}$. It is safe to say, then, that the prepulse is virtually eliminated. Fig. 6.24 shows the $\mathrm{K} \alpha$ conversion efficiency results from Titan versus LULI.

The coupling into $\mathrm{K} \alpha$ (normalized to the laser energy) was found to be approximately a factor of two higher than seen on Titan at the lowest prepulse level! Of course, at the moment, it is a bit difficult to decouple the impact of having practically no prepulse from the physics of electron generation using a $2 \omega_{0}$ (and therefore lower peak intensity and lower $I \lambda^{2}$ ) laser, but the fact that the coupling is higher is promising, and is perhaps an indication that fast ignition will need to move towards a $2 \omega_{0}$ laser design in order to attain the conversion efficiencies needed.

The experiments and modeling described here have provided valuable insight about the physics of relativistic laser interaction and electron generation inside a cone and electron propagation out beyond the cone tip. We have presented here a simultaneous scaling of both conversion efficiency and $T_{h o t}$ as a function of prepulse level in cone-wire targets. From these results, there is an evident drop off in coupling into forward-going fast electrons with increasing prepulse levels, as much as a factor of 8 in going from an $8 \mathrm{~mJ}$ to $1 \mathrm{~J}$ of prepulse, at Titan laser conditions. No evidence was seen of preplasma sweeping by the main pulse. This points towards the necessity to minimize prepulse levels to optimize coupling. Chapter 6 is material currently being prepared for publication by the dissertation author. 


\section{Chapter 7}

\section{Conclusions and Future Work}

\subsection{Summary of the Work}

In this thesis, the development and qualification of an Extreme Ultraviolet (XUV) imager at $68 \mathrm{eV}$ and $256 \mathrm{eV}$ for the measurement of electron-heated target temperatures has been completed. The multilayer mirrors used to preferentially image over a small bandwidth have been fully calibrated, and the mirror reflectivities as a function of incident angle, incident energy, reflection order, and accumulation of debris on the mirror face have been established. The $68 \mathrm{eV}$ spherical (focusing) and planar (translating) mirrors were found to be fairly accommodating in the incidence angle over which energies between 68 and $69 \mathrm{eV}$ would will be optimally reflected. However, the $256 \mathrm{eV}$ mirrors were found to be slightly more sensitive, where a small shift of a few degrees in incidence angle could move the reflectivity peak by several $\mathrm{eV}$. The decrease in reflectivity due to debris deposition on the front face of the spherical mirrors was found to be far more pronounced for the $68 \mathrm{eV}$ mirrors than the $256 \mathrm{eV}$ mirrors ( $40 \%$ decrease versus $2 \%$ over the same series of exposure to blow-off debris from the laser-plasma interaction).

Both channels of the XUV diagnostic were then set up on the Titan Laser facility, and simultaneously used to image short pulse laser-irradiated thin foils. LASNEX radiation-hydrodynamics modeling of the rear plasma expansion and emergent XUV spectral intensity were used to derive rear surface temperatures of $\mathrm{Cu}$ and $\mathrm{Al} / \mathrm{Cu}$ planar targets varying in thickness from $10 \mu \mathrm{m}$ to $40 \mu \mathrm{m}$. The peak 
rear surface temperatures for these thin foil targets was found to vary between 75 and $225 \mathrm{eV}$, with thinner targets exhibiting a higher level of heating. The inferred temperature based on each channel of the imager were validated against each other and found to match up to within $40 \mathrm{eV}$.

XUV spectroscopy in the 50 - $400 \mathrm{eV}$ region was additionally utilized to provide further temperature information using a completely independent method. Rear surface emission spectra from a $25 \mu \mathrm{m}$ deuterated carbon (CD) planar target irradiated at $144 \mathrm{~J}, 0.5 \mathrm{ps}, 50 \mu \mathrm{m}$ focal spot was analyzed. The ratios of hydrogenic to helium-like carbon lines integrated over a region of the rear surface plasma plume were determined. Comparison of the experimental spectra to synthetic spectra generated using the FLYCHK 0-D atomic spectroscopy code gave an average temperature of $74 \mathrm{eV}$ in an area of the plasma plume $300 \mu \mathrm{m}$ thick, expanded 300 $\mu \mathrm{m}$ from the rear surface. The peak temperature at the rear surface, as inferred through XUV 2-D imaging, was found to be $70 \mathrm{eV}$ higher. This provided an idea of the cooling rate of the plasma as it expanded outward from the target rear.

The imagers have since been applied to multiple parts of the fast ignition program, and have been found to be very useful in not only determining target temperatures but to provide high resolution 2-D images of the plasma heating.

An experiment conducted on nail-wire targets $(20 \mu \mathrm{m}$ diameter copper wires with $80 \mu \mathrm{m}$ hemispherical head) using the Vulcan Petawatt Laser (300 J, 1 ps, $2 \times 10^{20}$ $\mathrm{W} / \mathrm{cm}^{2}$ ) was undertaken to try to understand the transport of relativistic electrons in a solid target. A spherically bent $\mathrm{Cu} \mathrm{K} \alpha$ crystal imager, a HOPG spectrometer, and a single photon counting $\mathrm{CCD}$ gave absolute $\mathrm{Cu} \mathrm{K} \alpha$ measurements. Results showed a concentration of energy deposition in the head and an approximately exponential fall-off along the wire with about $60 \mu \mathrm{m} 1 / e$ decay length due to resistive inhibition. The coupling efficiency to the wire was $3.3 \pm 1.7 \%$ with an average hot electron temperature of $520 \pm 125 \mathrm{keV}$. XUV images at 68 and 256 $\mathrm{eV}$ showed limb brightening, indicating additional heating of a thin surface layer of the wire.

The simple quasi-1-D geometry of the target allowed for concurrent bench- 
marking of the ePLAS hybrid transport code. Modeling was compared with the experimental data, showing evidence of resistive heating, magnetic trapping, and surface transport.

The nail-wire was novel in that it provided one-dimensional (along electron propagation axis) K $\alpha$ measurements to simultaneously give information on the energy distribution and laser-to-electron coupling efficiency without the complexities of a diverging electron beam. In earlier experiments, electron flux had been characterized through the use of buried fluor layers in planar targets, where the fluor layer was systematically moved back with each shot to construct a full picture of the energy deposition as a function of depth. However, these nail-wire targets helped to demonstrate that it was possible within one single shot to obtain the same information, without the complications of shot-to-shot variation and using up several laser shots.

This technique was then applied to cone-wire targets, in which Au cones $\left(30^{\circ}\right.$ full opening angle, $20 \mu \mathrm{m}$ thick cone walls, $11 \mu \mathrm{m}$ thick cone tip, $30 \mu \mathrm{m}$ inner cone tip diameter) coupled to thin $40 \mu \mathrm{m} \mathrm{Cu}$ wires were imaged to obtain a scaling of coupling into forward-propagating electrons as a function of prepulse level into the cone. Previous studies had only provided a qualitative assessment of the consequence of preformed plasma inhibiting energy coupling beyond the tip of the cone, but this work provided the first quantitative scaling of coupling and $T_{h o t}$ as a function of prepulse level.

The HYDRA radiation-hydrodynamics code was used to model the preplasma formation inside the cone, and even in the cases of small $(\sim 8 \mathrm{~mJ})$ prepulse, the growth of a strong on-axis jet was obvious. This jet of higher density plasma on the axis of the cone was due to preplasma expanding outward from the cone walls, and colliding on-axis. This effect and the effect of preplasma filling of the cone was stronger the larger the prepulse energy, pushing the critical surface (the general location of absorption of laser energy into energetic electrons) farther back from the tip of the cone.

Zuma, a hybrid transport code that combines collisional Monte Carlo with field 
generation, was then employed to model the $\mathrm{K} \alpha$ emission along the wire in order to infer the hot electron population and energy distribution. The coupling into the first $500 \mu \mathrm{m}$ of the wire was found to be on the order of $1 \%$ for the minimum prepulse case on Titan $(8 \mathrm{~mJ})$, and decreased by a factor of 8 to below $0.2 \%$ for the case of $1 \mathrm{~J}$ of injected artificial prepulse. The temperature of the electron distribution was not found to vary significantly (400 - 500 keV) across the large range of prepulse levels, signifying little or no effects of ponderomotive steepening. This work demonstrates the dominating role that preplasma in the cone plays on the coupling into hot electrons that can propagate beyond the cone.

\subsection{Future Experiments and Modeling}

Until a full-scale integrated fast ignition experiment can be done, small parts of the fast ignition problem will have to be tackled through a combination of simulation, theory, and experimental elements. Experiments can be carefully designed to learn about a particular aspect of the key physics (such as electron divergence or transport), while providing systematic benchmarking of the simulation codes. In this way, the process can be progressively iterated to develop more sophisticated modeling tools and a better understanding of the physical mechanisms at work. Then, as the codes improve, so will their predictive capability for modeling full-scale FI.

In the meantime, cone-wire targets will continue to serve as just such a useful experimental diagnostic for benchmarking the fast ignition models. The cone-wires act as a relevant fast ignition surrogate, where the cone is similar to the reentrant fast ignition cone, and the wire is a proxy for the dense core beyond. Through the work here, it has been demonstrated that cone-wires are worthwhile tools for characterizing the electron source and subsequent transport, and efforts continue on experimentally pinpointing $\eta$ and $\mathrm{T}_{h o t}$. The possibilities for experimental investigation using cone-wires in various forms is numerous: different cone opening angles, cone materials, laser irradiation conditions, etc. This will provide highly valuable information in defining the final fast ignition target design. 
The interpretation of these experiments is highly dependent on modeling, so the thrust to constantly improve the simulation codes is strong also for that reason. One principal goal is to develop a flexible modeling platform that encompasses radiation-hydrodynamics and laser absorption and fast electron generation, transport and stopping. A sizable step towards that, and an obvious extension from the work in this thesis will be to model the cone-wire experiment at full spatial and temporal scales. As in the previous simulations of isolated cone targets by MacPhee et al.[97], HYDRA radiation-hydrodynamics will be used to establish the initial preplasma conditions, and then the PSC+Hybrid PIC code will be used to self-consistently model the full laser-plasma interaction in the underdense plasma, the absorption of the laser energy near critical density, and subsequent transport of the fast electrons into the wire. This will be a considerable refinement over the Zuma modeling utilized here, as it will be a forward calculation starting from a realistic laser pulse within the cone step by step all the way through to the energy deposition in the wire, rather than a backward calculation to infer the properties of the initial laser-target interaction.

The work, of course, continues until both numerical modeling and experiment can converge on a full-scale, integrated, successful fast ignition demonstration.

\subsection{Concluding Remarks}

Fast ignition is an advanced scheme for inertial confinement fusion with the potential for a lower ignition threshold, less stringent implosion symmetry requirements, and higher gain over central hot spot ignition. If realized, fast ignition may help promote the viability of inertial fusion as a useful energy source. Numerous key issues remain, however, including how to achieve the $300 \mathrm{~g} / \mathrm{cm}^{3}$ density and 2 $\mathrm{g} / \mathrm{cm}^{2} \rho \mathrm{R}$ in the implosion phase, maintaining the integrity of the cone and cone tip in the hydro implosion so that a clear path is available for the short pulse laser, minimizing pre-heat and ablation of the cone that can lead to mixing with the fuel, and minimizing the distance from the compressed core to the cone tip so that fast electron coupling can be maximized. In this dissertation, only a small piece of 
the FI puzzle has been examined, and perhaps the most appropriate conclusion is but to say that relativistic laser absorption and fast electron transport is extremely complex, and a good deal of effort remains in order to gain a better understanding. 


\section{Bibliography}

[1] Lindl, J., Phys Plasmas (1995).

[2] White, R., Resler, D., and Warshaw, S., ... on Nuclear Data for Science and ... (1991).

[3] Atzeni, S. and Meyer-Ter-Vehn, J., The Physics of Inertial Fusion: Beam Plasma Interaction, Hydrodynamics, Hot Dense Matter, Oxford University Press, 2004.

[4] Rosen, M., Phys Plasmas (1999).

[5] Fraley, G., Linnebur, E., Mason, R., and Morse, R., Physics of Fluids 17 (1974) 474 .

[6] Tabak, M., Hammer, J., Glinsky, M., Kruer, W., and Wilks, S., Phys Plasmas (1994).

[7] Tabak, M., Hinkel, D., Atzeni, S., Campbell, E., and Tanabe, K., Fusion Sci Technol 49 (2006) 254.

[8] Atzeni, S., Phys Plasmas (1999).

[9] Li, C. and Petrasso, R., PHYSICAL REVIEW E Phys Rev E 70 (2004) 067401 .

[10] Norreys, P., Allott, R., Clarke, R. J., and ..., J. C., Phys Plasmas (2000).

[11] Hatchett, S. and Tabak, M., Proc. 30th Annual Anomalous Absorption Conf. (2000) 1.

[12] Hatchett, S. et al., Fusion Sci Technol 49 (2006) 327.

[13] Kodama, R., Norreys, P., Mima, K., Dangor, A., and Evans, R., Nature (2001).

[14] King, J. et al., Phys Plasmas 16 (2009) 020701. 
[15] Chen, F., Introduction to Plasma Physics and Controlled Fusion, Volume 1: Plasma Physics, Plenum Press, 2nd edition, 1984.

[16] Kruer, W., The Physics of Laser Plasma Interactions, Addison-Wesley, 1988.

[17] Max, C. E., Physics of Laser Fusion Volume I: Theory of the Coronal Plasma in Laser Fusion Targets, Lawrence Livermore National Laboratory, 1982.

[18] Ginzberg, V. L., The Properties of Electromagnetic Waves in Plasma, Pergamon, New York, 1964.

[19] Freidberg, J., Mitchell, R., Morse, R., and Rudsinski, L., Phys. Rev. Lett. 28 (1972) 795.

[20] Forslund, D., Kindel, J., Lee, K., Lindman, E., and Morse, R., Physical Review A 11 (1975) 679.

[21] Estabrook, K. and Kruer, W., Phys. Rev. Lett. 40 (1978) 42.

[22] Brunel, F., Phys. Rev. Lett. 59 (1987) 52.

[23] Gibbon, P. and Bell, A., Phys. Rev. Lett. 68 (1992) 1535.

[24] Kruer, W. and Estabrook, K., Physics of Fluids 28 (1985) 430.

[25] Wilks, S., Kruer, W., Tabak, M., and Langdon, A., Phys. Rev. Lett. (1992).

[26] Malka, G. and Miquel, J., Phys. Rev. Lett. 77 (1996) 75.

[27] Wharton, K., Hatchett, S., Wilks, S., and Key, M., Phys. Rev. Lett. (1998).

[28] Yasuike, K., Key, M., Hatchett, S., Snavely, R., and Wharton, K., Rev. Sci. Instrum. 72 (2001) 1236.

[29] Chen, C. D., Patel, P., Hey, D., and Mackinnon, A., Phys Plasmas (2009).

[30] Freeman, R., Batani, D., Baton, S. D., Key, M., and Stephens, R., Fusion Sci Technol 49 (2006) 297.

[31] Key, M., Cable, M., ..., T. E. C., and Estabrook, K., Phys Plasmas (1998).

[32] Beg, F., Bell, A., Dangor, A., Danson, C., and Fews, A., Phys Plasmas (1997).

[33] Clark, E. et al., Phys. Rev. Lett. 85 (2000) 1654.

[34] Haines, M., Wei, M., Beg, F., and Stephens, R., Phys. Rev. Lett. 102 (2009) 045008 . 
[35] Kemp, A., Sentoku, Y., and Tabak, M., Phys. Rev. Lett. (2008).

[36] Chrisman, B., Sentoku, Y., and Kemp, A., Phys Plasmas (2008).

[37] Davies, J. R., Physical Review E (2002).

[38] Batani, D., Laser Part Beams 20 (2002) 321.

[39] Nardi, E. and Zinamon, Z., Physical Review A 18 (1978) 1246.

[40] Atzeni, S., Schiavi, A., and Davies, J. R., Plasma Phys Contr F (2009).

[41] Bond, D., Hares, J., and KILKENNY, J., Phys. Rev. Lett. 45 (1980) 252.

[42] Bell, A., Davies, J. R., Guerin, S., and Ruhl, H., Plasma Phys Contr F 39 (1997) 653.

[43] Spence, D., Kean, P., and Sibbett, W., Optics Letters 16 (1991) 42.

[44] Joosen, W., Bakker, H., Noordam, L., Muller, H., and Heuvell, H., Journal of the Optical Society of America B 8 (1991) 2537.

[45] Strickland, D. and Mourou, G., Opt Commun 55 (1985) 447.

[46] Baumgartner, R. and Byer, R., IEEE Journal of Quantum Electronics QE15 (1979) 432.

[47] Cerullo, G. and Silvestri, S. D., Rev. Sci. Instrum. (2003).

[48] Dubietis, A., Jonusauskas, G., and Piskarskas, A., Opt Commun 88 (1992) 437.

[49] Ross, I., Matousek, P., Towrie, M., Langley, A., and Collier, J., Opt Commun 144 (1997) 125.

[50] Jovanovic, I., Comaskey, B., Ebbers, C., and Bonner, R., Appl. Opt (2002).

[51] Goldstein, R., Laser Focus (1968).

[52] Caird, J. et al., (2004).

[53] Stuart, B., Bonlie, J., Britten, J., and Caird, J., ... 2006 Quantum Electronics and Laser Science Conference. CLEO ... (2006).

[54] Danson, C. et al., Nuclear Fusion 44 (2004) S239.

[55] Musgrave, I., Hernandez-Gomez, C., Canny, D., and ..., J. C., Appl Optics (2007). 
[56] Trebino, R., Frequency-Resolved Optical Gating: The Measurement of Ultrashort Laser Pulses, Kluwer Academic Publishers, 2000.

[57] Diels, J., Fontaine, J., McMichael, I., and Simoni, F., Appl Optics 24 (1985) 1270 .

[58] MacPhee, A., Akli, K. U., Beg, F., Chen, C. D., and Chen, H., Rev. Sci. Instrum. (2008).

[59] Pape, S. L., Tsui, Y., MacPhee, A., and Hey, D., Optics Letters (2009).

[60] Gu, P. et al., Rev. Sci. Instrum. 77 (2006) 113101.

[61] Ma, T., Beg, F., MacPhee, A., Chung, H.-K., and Key, M., Rev. Sci. Instrum. (2008).

[62] Tokushima, T. et al., Surface Review and Letters 9 (2002) 503.

[63] Neely, D., Damerell, A., Parker, R., and Zepf, M., (2003) 2.

[64] Hombourger, C., JOURNAL OF PHYSICS-LONDON-B ATOMIC MOLECULAR AND OPTICAL PHYSICS 31 (1998) 3693.

[65] Koch, J. et al., Rev. Sci. Instrum. 74 (2003) 2130.

[66] Akli, K. U., Key, M., Chung, H.-K., Hansen, S., and Freeman, R., Phys Plasmas (2007).

[67] Pak, A. et al., Rev. Sci. Instrum. 75 (2004) 3747.

[68] Legall, H., Stiel, H., Arkadiev, V., and Bjeoumikhov, A., Opt Express 14 (2006) 4570.

[69] del Rio, M. et al., Proceedings of SPIE 3448 (1998) 246.

[70] Legall, H. et al., Proceedings of SPIE 5918 (2005) 591802.

[71] Stoeckl, C. et al., Rev. Sci. Instrum. 75 (2004) 3705.

[72] Maddox, B., Park, H., Remington, B., and Mckernan, M., Rev. Sci. Instrum. 79 (2008) 10E924.

[73] Higginson, D. P., internal memo (2010) 1.

[74] Kodama, R., Okada, K., and Kato, Y., Rev. Sci. Instrum. 70 (1999) 625.

[75] Stephens, R. et al., PHYSICAL REVIEW E Phys Rev E 69 (2004) 066414.

[76] Shen, M.-Y. et al., Optics and Photonics Conference, Taiwan, 2001 (2001) 1. 
[77] Planck, M., Annalen der Physik (1901).

[78] Attwood, D., Soft X-Rays and Extreme Ultraviolet Radiation: Principles and Applications, Cambridge University Press, 1999.

[79] Barbee, T., Optical engineering(Bellingham. Print) 25 (1986) 898.

[80] Underwood, J. et al., Rev. Sci. Instrum. 67 (1996) 3372.

[81] Zimmerman, G., Kershaw, D., Bailey, D., and Harte, J., Inertial Confinement Fusion Conference (1978) 1.

[82] Chen, H. and Wilks, S., Laser Part Beams 23 (2005) 411.

[83] Chung, H., Chen, M., Morgan, W., Ralchenko, Y., and LEE, R., High Energy Density Physics 1 (2005) 3.

[84] McKee, G., Fonck, R., Stratton, B., Budny, R., and ..., Z. C., Nuclear Fusion (1997).

[85] Green, J. S. et al., Nat Phys 3 (2007) 853.

[86] Kodama, R., Azechi, H., Fujita, H., Habara, H., and Izawa, Y., Nucl. Fusion (2004).

[87] Wei, M., Solodov, A., Pasley, J., and Stephens, R., Phys Plasmas (2008).

[88] Bastiani, S. et al., Physical review. E, Statistical physics, plasmas, fluids, and related interdisciplinary topics 60 (1999) 3439.

[89] Ma, T., MacPhee, A., Key, M., Hatchett, S., and Akli, K. U., Rev. Sci. Instrum. (2008).

[90] Lee, Y. and More, R., Physics of Fluids 27 (1984) 1273.

[91] Chung, H.-K., Chen, M. H., and LEE, R., High Energy Density Physics 3 (2007) 57.

[92] Mason, R. and Cranfill, C., IEEE Transactions on Plasma Science 14 (1986) 45.

[93] Mason, R., Wei, M., Beg, F., Stephens, R., and Snell, C., Pulsed Power Plasma Science Conference (2007).

[94] Norreys, P., Beg, F., Sentoku, Y., and Silva, L., Phys Plasmas (2009).

[95] Woerkom, L. V., Akli, K. U., Bartal, T., Beg, F., and ..., S. C., Phys Plasmas (2008). 
[96] Baton, S. et al., Phys Plasmas 15 (2008) 042706.

[97] Macphee, A. G. et al., Phys. Rev. Lett. 104 (2010) 055002.

[98] Kemp, A. J., Cohen, B. I., and Divol, L., Phys Plasmas 17 (2010) 056702.

[99] Ma, T. et al., Phys Plasmas 16 (2009) 112702.

[100] Honrubia, J. and Antonicci, A., ... and Particle Beams (2004).

[101] Theobald, W. et al., Phys Plasmas 13 (2006) 043102.

[102] Marinak, M., Kerbel, G., Gentile, N., and Jones, O., Physics of ... (2001).

[103] Akli, K., Stephens, R., and Key, M., IOP Physics: International Fusion Sciences Conference (2010) 1. 